\title{
Biomarkers of mineral dust induced lung disorders : molecular epidemiologic studies in coal workers
}

Citation for published version (APA):

Schins, R. P. F. (1996). Biomarkers of mineral dust induced lung disorders : molecular epidemiologic studies in coal workers. [Doctoral Thesis, Maastricht University]. Universiteit Maastricht. https://doi.org/10.26481/dis.19961030rs

Document status and date:

Published: 01/01/1996

DOI:

10.26481/dis.19961030rs

Document Version:

Publisher's PDF, also known as Version of record

\section{Please check the document version of this publication:}

- A submitted manuscript is the version of the article upon submission and before peer-review. There can be important differences between the submitted version and the official published version of record.

People interested in the research are advised to contact the author for the final version of the publication, or visit the DOI to the publisher's website.

- The final author version and the galley proof are versions of the publication after peer review.

- The final published version features the final layout of the paper including the volume, issue and page numbers.

Link to publication

\footnotetext{
General rights rights.

- You may freely distribute the URL identifying the publication in the public portal. please follow below link for the End User Agreement:

www.umlib.nl/taverne-license

Take down policy

If you believe that this document breaches copyright please contact us at:

repository@maastrichtuniversity.nl

providing details and we will investigate your claim.
}

Copyright and moral rights for the publications made accessible in the public portal are retained by the authors and/or other copyright owners and it is a condition of accessing publications that users recognise and abide by the legal requirements associated with these

- Users may download and print one copy of any publication from the public portal for the purpose of private study or research.

- You may not further distribute the material or use it for any profit-making activity or commercial gain

If the publication is distributed under the terms of Article $25 \mathrm{fa}$ of the Dutch Copyright Act, indicated by the "Taverne" license above, 


\section{Biomarkers of Mineral Dust Induced Lung Disorders}

Molecular epidemiologic studies in coal workers 
(a) Schins, Maastricht 1996

ISBN 9090099220

Omslag ontwerp: Datawyse Boekprodukties. 


\section{Biomarkers of Mineral Dust Induced Lung Disorders}

Molecular epidemiologic studies in coal workers

\section{Proefschrift}

ter verkrijging van de graad van doctor aan de Universiteit Maastricht, op gezag van de Rector Magnificus, Prof. mr. M.J. Cohen, volgens het besluit van het College van Dekanen, in het openbaar te verdedigen op woensdag 30 oktober 1996 om 16.00 uur

door

Peter Frans Roeland Schins

geboren te Heerlen op 27 juli 1966 


\section{Promotor:}

Prof. dr. J.C.S. Kleinjans

\section{Co-promotor:}

Dr. P.J.A. Borm

\section{Beoordelingscommissie:}

Prof. dr. E.F.M. Wouters (voorzitter)

Prof. dr. A. Bast (Vrije Universiteit Amsterdam)

Dr. W.A. Buurman

Prof. dr. J.M.A. van Engelshoven

Dr. P.A. Schulte (National Insititute for Occupational Safety and Health, Cincinnati, OH, USA)

The studies presented in this thesis were financially supported by the European Committee for Steel and Coal (EC-ECSC grant no. 7263/03/092) 
sjtub $m 1$ stof. Ziech oes d'r - maache, zich uit de voeten maken. 2 stoflongen, silicose.

(Kirchröadsjer Dieksiejoneer) 


\section{Contents}

\section{Abbreviations 8}

Chapter 1 General introduction and aims of the studies 9

1.1 Minerals and health effects 9

1.2 Biological markers 13

1.3 Aim of the thesis 15

Chapter 2 Coal dust induced lung disorders: Epidemiology 17

2.1 Historical background 17

2.2 Coal dust 28

2.3 Coal workers pneumoconiosis 19

2.4 Chest radiography examination of pneumoconiosis 21

2.5 Nonpneumoconiotic effects of coal dust 22

Chapter 3 Coal dust induced lung disorders: Mechanisms 25

3.1 Particles in the lung: the role of phagocytes, epithelial cells, and fibroblasts 25

3.2 Oxidative damage and antioxidant defense 28

Reactive oxygen species and antioxidants 28

Reactive oxygen species and antioxidants in mineral dust induced lung disorders 31

3.3 Cytokines 34

Cytokines and mineral dust 34

Tumor necrosis factor- $\alpha$ and its receptors 37

Transforming growth factor $\beta \quad \beta 39$

Other cytokines relevant to mineral dust induced effects 40

Cytokine networks in mineral dust induced lung disorders

3.4 Collagens 43

Collagens in the lung 43

Collagen formation and degradation 44

Type III procollagen peptide 45

Chapter 4 Design of the studies 47

4.1 Description of the study population and

biomarkers evaluated 47

4.2 Peripheral blood versus the lung 49 


\section{PART 1 Oxidative damage and antioxidant defense}

Chapter 5 Serum total radical-trapping antioxidant parameter (TRAP) in coal workers 53

Chapter 6 Oxidative DNA-damage in peripheral blood lymphocytes of coal workers 57

Chapter 7 Blood antioxidant status in coal dust induced respiratory disorders: a longitudinal evaluation of multiple markers 79

\section{PART 2 Cytokines}

Chapter 8 Epidemiological evaluation of release of monocyte TNF- $\alpha$ as an exposure and effect marker in pneumoconiosis: a five year follow up study among coal workers 79

Chapter 9 Plasma levels of the soluble Tumor Necrosis Factor (TNF) receptors are increased in coal miners with pneumoconiosis 99

Chapter 10 Multiple cytokines as biomarkers in coal dust exposure and pneumoconiosis: Tumor necrosis factor- $\alpha$, interleukin- 6 , and transfroming growth factor- $\beta \quad 109$

\section{PART 3 Collagen}

Chapter 11 Serum procollagen type III peptide in Coal Worker's pneumoconiosis: A five year follow-up study 123

Chapter 12 Evaluation of serum procollagen type III peptide as an exposure marker in retired coal workers 133

Chapter 13 Summary and general discussion 145

References 157

Samenvatting 179

Dankwoord 185

Curriculum vitae 187

List of publications 189 


\section{Abbreviations}

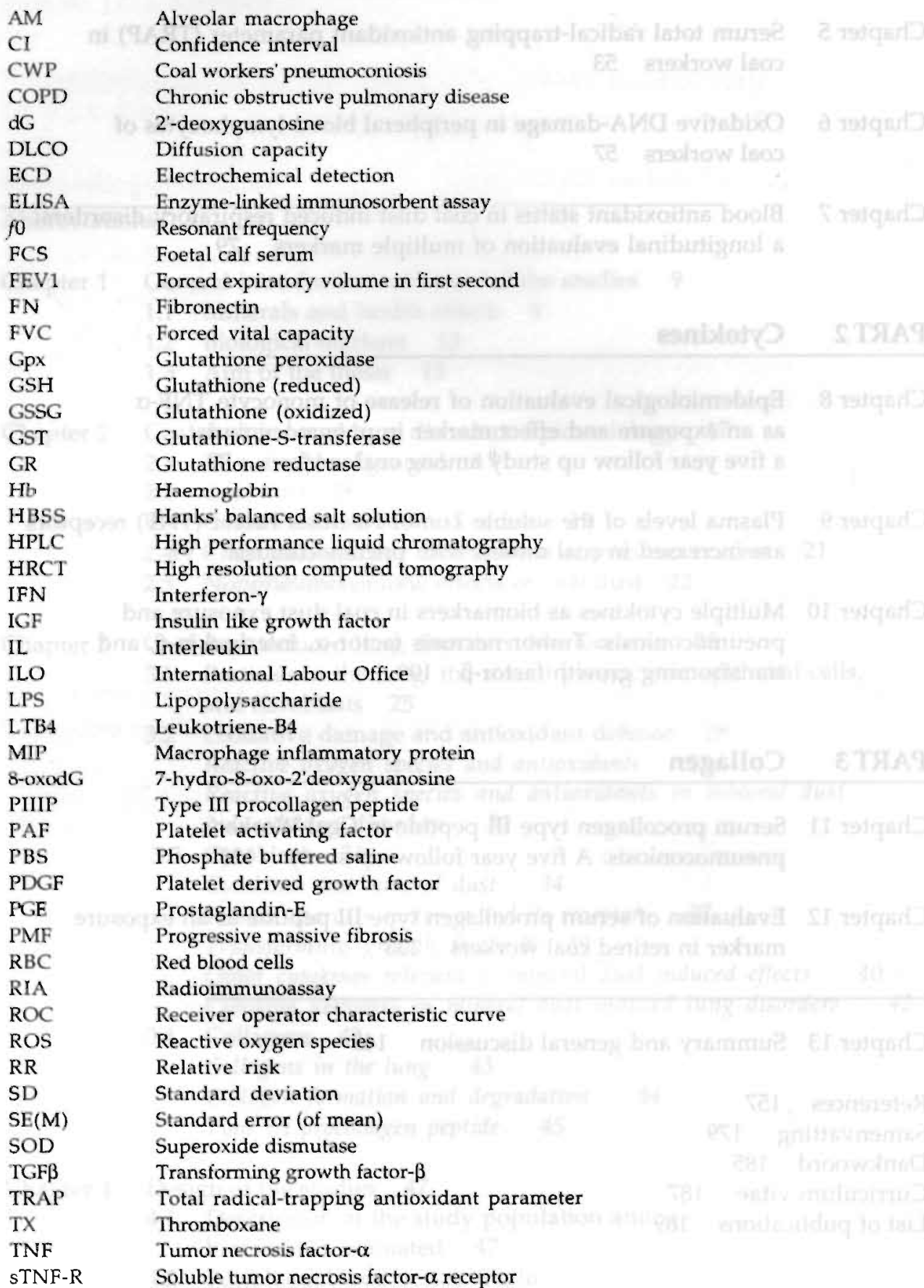




\subsection{Minerals and health effects}

Already in the ancient history man realized that the earth crust was rolling in minerals and ores to create tools, pottery and ceramics, or to construct buildings. Remains of stone buildings found near Jericho date back to 9000 B. C. and in Syria tools and ceramics have been excavated originating from Ugarit (6600 B.C.) and Mari (4500 B.C.) cultures. Egyptians became famous for their sculptured statues and reliefs, and their skilfulness to create beautiful objects of glass, enamel, and metals including gold. The earliest mining activities in the Netherlands date back to 3000 B.C., when flint was exploited and processed in the Southern part of the province Limburg (near the village of St. Geertruid).

Among the various minerals on earth, silica $\left(\mathrm{SiO}_{2}\right)$ is the most abundant. Other minerals include limestone (e.g. marble, dolomite), coal (e.g. anthracyte, graphite), asbestos fibers, and clay (e.g. kaolin or china clay, mica). Silica is present in granite, feldspar, sandstone, and shale. Shale is often found in the vicinity of coal deposits, and consists of silica, kaolinite, mica and may occasionally contain oil. Natural silica may occur as $\alpha$-quartz, cristoballite and tridymite. Diatomateous earth (amorphous silica) calcinated at $800^{\circ} \mathrm{C}$ to $1000^{\circ} \mathrm{C}$ is known as kieselguhr. Limestone is used for the production of cement and calcium(hydr)oxide and clays are principal resources for ceramic and pottery industry. Asbestos is a common name for naturally occurring mineral silicate fibers, which have been used extensively because of their great strength and heat resistance. Examples include chrysotile (serpentine fibers), amosite, crocidolite, anthophyllite, tremolite, and actinolite. Naturally occurring non-asbestos fibers include erionite (zeolite), attapulgite, wollastonite, sepiolite and graphite. Manmade fibers may be subdivided into mineral fibers (e.g. glassfibers, rockwool, and slagwool and ceramic fibers), and synthetic fibers (e.g. polyolefin fibers).

Despite the world-wide diversity in abundancy of mineral-rich areas and in the structure, physio-chemical properties and related applications of these different minerals, they all share the probability to become airborne as dusts and eventually inhaled. Dusts are defined as small, solid particles formed by the mechanical attrition or disintegration of larger masses of material [Cotes et al., 1987]. In addition to the minerals described above, dust can be generated from metal sources (e.g. iron, tin). Examples of organic dusts are wood-, cotton-, grainand flour-dust, and the (synthetic organic) aramid fibers.

Deposition of inhaled particles in the lung is primarily dependent on size, shape and particle density, as well as on individual lung morphology (e.g. lung volume) and physiology (breathing rate and depth) [Bohning and Lippmann, 
1992]. Generally, large particles $(>10 \mu \mathrm{m})$ will be filtered out of the inhaled airstream by the aerodynamic filters of the respiratory tract, whereas smaller particles are deposited in the tracheobronchial tree or alveoli [Churg et al., 1985; Clarke and Pavia, 1991]. Fibershaped particles have dissimilar deposition patterns related to their specific length-diameter characteristics [Lippmann, 1988]. In the upper airways, dust particles are cleared by mechanisms of sneeze and cough, or by the mucocilliar escalator, and ultimately be swallowed [Clarke and Pavia, 1991; Bohning and Lippmann, 1992]. In the alveolar region, the majority of the particles are ingested by phagocytotic cells such as macrophages and neutrophils. Particles may be cleared by translocation via the alveolar epithelium upstream for mucocilliar clearance, or to the interstitium for clearance by interstitial macrophages via the regional lymph nodes [Bohning and Lippmann, 1992].

Intrinsic (chemical, morphological) properties of the dust denominate its durability and the rate to which a particle is dissolved or degraded in the lungs. Durability can range from days (e.g. cement dust) to decades (e.g. asbestos). If a particle is degradable, the alveolar macrophage will resume its normal function. However, if particles are non-degradable, the alveolar macrophage is either removed via clearance on the epithelial surface or interstitium, or remains in situ with the particle. If the diameter, or in the case of fibershaped material, the length of a particle is very large, "frustrated phagocytosis" may occur characterized by an incomplete phagocytosis [Mossman et al., 1991].

A state of "particle overload" may occur if more particles enter the lung than can be cleared from it due to impairment of macrophage clearance [Bolton, 1983; Morrow, 1992]. Thus, relatively harmless dusts can cause damage of the alveolar epithelium, and particles may reach the interstitium [Driscoll et al., 1990]. Utrafine particles are considered to cross the epithelial barrier into the interstitium more readily [Oberdörster et al., 1994] and noteworthy, a relative low weight-dose of ultrafine particles may comprise a tremendous numerical dose. The cumulative inhaled dose is of major importance in (chronic) effects of any particulate matter. Although the hazard of different particles may vary considerably, at sufficient high dust concentrations, all dusts are considered capable of producing irreversible lung changes [Gross, 1967].

Already in ancient time it was realized that mining and stone cutting could cause adverse health effects, and it was literally considered as slave labour. In 1556 the book "De Re Metallica" written by Agricola (1494-1555) became published. This book included an advice on how to improve working conditions of miners. In 1806, Laennec recognized a disease in the lungs of coal miners which he described as melanosis [Cotes et al., 1987]. Nowadays it is known that mineral dusts may cause a variety of lung disorders. In Table 1.1, some examples of mineral dusts and related diseases are listed. It should be stressed that the causality of some of the disorders as forwarded from several studies is still subject to debate, and regularly rejected by several investigators. For instance, it is 
accepted that asbestos fibers may cause bronchogenic carcinoma or mesothelioma, a malignant disease of the pleura [Mossman et al., 1989], while silica currently is classified as a "suspect" carcinogen by the International Agency for Research on Cancer [IARC, 1987], and was recently forwarded as a carcinogen to humans by the Dutch expert committee [DECOS, 1996]. Additionally, gastrointestinal malignancies have occasionally been observed in mortality studies in mineral dust exposed cohorts, and are associated with the mucociliar clearance route of inhaled particles [Meyer et al., 1980]. Regarding obstructive lung diseases observed in subjects chronically exposed to mineral dust, it is now realized that confounding effects of smoking should be seriously taken into account [Oxman et al., 1993; Weill, 1993].

Table 1.1 Examples of mineral dusts and disorders associated with their inhalation

\begin{tabular}{llll} 
Dust & Pneumoconiotic & Nonpneumoconiotic & Malignant disease \\
\hline Asbestos & $\begin{array}{l}\text { Asbestosis } \\
\text { [Cooke, 1927] } \\
\text { PMF }\end{array}$ & $\begin{array}{l}\text { Lung function loss } \\
\text { [Zedda et al., 1973] }\end{array}$ & $\begin{array}{l}\text { Lung cancer } \\
\text { [Mossman et al., 1990] } \\
\text { [Mossman et al., 1990] }\end{array}$ \\
[Wagnet et al., 1960]
\end{tabular}

PMF: progressive massive fibrosis; CWP : coal workers pneumoconiosis 
Inherent to its definition, pneumoconiosis is considered as a common feature of chronic inhalation of any dust. Current definition of pneumoconiosis is "the accumulation of dust in the lungs and the tissue reactions to its presence" [Gross, 1983]. The word pneumoconiosis was introduced in 1867 by the German pathologist Zenker, based on the Greek words for lung and dust [Meijers, 1991]. Pneumoconiosis caused by inhalation of mineral dusts have been described in various occupational settings such as granite, ceramic and pottery industries, mining and grinding of coal, asbestos, kaolin, gold, tin, and sandblasting [Cotes et al., 1987; Cullen et al., 1990]. Knowledge of the individual's (occupational) exposure history is a prerequisite to clinical examination of pneumoconiosis. A subject with radiological indication of pneumoconiosis is considered to have coal workers pneumoconiosis (CWP), in the knowledge that he has been exposed to coal dust. Similarly, in subjects chronically exposed to free silica, silicosis may develop, and asbestos exposure may lead to asbestosis. Examples of other minerals or metals that can cause pneumoconiosis are talc (talcosis), iron (siderosis), tin (stannosis), mica, tungsten, barite (baritosis), kaolin (kaolinosis), attapulgite, wollastonite and sepiolite [Rom, 1992a; Cotes et al., 1987] (see Table 1.1). Mixed pneumoconiosis may develop in subjects with chronic exposure to more than one type of dust.

In spite of their common definition, the different pneumoconioses usually vary considerably in pathologic features. On chest radiographs of CWP and silicosis, lesions are visible throughout the lung, albeit particularly (and at earlier stage) in the upper lung zones. Asbestosis is characterized by lesions in the lower lobes of the lung, and in severe cases lesions are seen in subpleural tissue. Furthermore, chest radiographs of CWP and silicosis are more often characterized by rounded opacities, while asbestos mainly gives rise to irregular shaped opacities [Merchant and Schwartz, 1992]. Lung biopsy studies have yielded marked differences in structural features of the simple coal dust lesion (i.e. macule) and the silicotic nodule, especially with regard to connective tissue content [Heppleston, 1992]. The replacement of normal pulmonary parenchyme by a connective matrix is an irreversible process, and this fibrogenic accumulation is characteristic to fibrosis [Snyder, 1988; Murray and Laurent, 1988]. Compared to silicosis, fibrogenesis is relatively mild process in early stage coal workers pneumoconiosis. Other dusts (e.g. barite and titanium) are associated with a minimal or lacking fibrotic response, and usually cause reversible types of pneumoconiosis.

Pneumoconioses are generally divided into two major categories, i.e. simple pneumoconiosis and progressive massive fibrosis (PMF). In simple pneumoconiosis discrete small lesions are scattered throughout the lung in varying profusion and with varying degrees of fibrosis. In early stages of simple fibrosis physiological lung functions are usually not reduced in CWP or silicosis, in contrast to asbestosis. PMF is characterized by development of large lesions, 
with fibrosis and collapse of lung parenchyma. Very often the lesions may involve an entire lung lobe, which is reduced to a scar, with varying amounts of dust and calcification. In silicosis and CWP these severe lesions usually develop in the upper lobe, or there may be more than one discrete lesion. In asbestosis with PMF the lower lobes are more frequently involved. Lesions of PMF almost exclusively occur on a background of simple pneumoconiosis [Hurley et al., 1987; Snyder, 1988; Merchant and Schwartz, 1992].

It is now evident that chronic inhalation of mineral dusts may lead to a variety of disorders of the respiratory tract, and can reduce the quality or even quantity of life in those who have become diseased [Enterline, 1964; Cochrane 1973; Miller and Jacobson, 1985; IARC, 1987; Mossman at al., 1990; Dutch Health Council 1992; Oxman et al., 1993]. At present, mineral dust induced lung disorders are among the most widespread occupational lung diseases. Silicosis is considered the most prevalent chronic occupational disease in the world [Cullen et al., 1990]. As an example, in China 10 million people are thought to be exposed to mixed dusts, and in 1987 more than 600.000 pneumoconiosis patients were registered [Sjöstrand, 1989]. According to the NIOSH, occupational lung diseases, including pneumoconiosis and lung cancer, are considered as the leading work-related diseases, based on frequency of the problems, severity of disease and preventability [Rom, 1992b]. In 1989 in the United Kingdom, the SWORD project started, a reporting scheme for work related and occupational lung diseases formed by members of the British Thoracic Society [Seaton, 1991]. Major findings were that long latency disease risk was particularly high in construction workers, miners, shipyard and dock workers [Meredith and McDonald, 1994], and of all cases reported in $1989(\mathrm{n}=2101), 13 \%$ were pneumoconiosis [Seaton, 1991]. In mining industry, in spite of tremendous efforts to reduce exposure, pneumoconiosis has not yet ceased to exist [Cullen et al., 1990]. In the countries united in the (former) European committee for Steel and Coal (ECSC) coal dust induced lung disorders increasingly become a post-occupational problem [Soutar et al., 1986; Gautrin et al., 1994]. Mineral dust induced lung disorders are also regularly being reported in relation with other occupational exposures [Landrigan, 1987; Cullen et al., 1990; Nemery et al., 1992].

\subsection{Biological markers}

These facts (further) necessitate primary prevention (dust prevention), medical screening of exposed subjects, monitoring of exposure and post-exposure surveillance. Continued biomedical research on mineral dust induced lung disorders has allowed a better understanding of pathogenic mechanisms. Through these new insights tools may be generated to identify those at increased 
health risk, and for medical care in those who have become diseased. Exactly these insights are considered as the hallmarks of molecular epidemiology, i.e the incorporation of biomarkers in the field of "traditional" (occupational) epidemiology [Schulte, 1993]. Where epidemiology can be defined as "the study of the distribution and determinants of health-related states and events in populations and the application of the results in this study to control health problems", molecular epidemiology may be defined as "the incorporation of measurements of physiologic, cellular, subcellular and molecular events into epidemiologic research" [Schulte, 1993]. These measurements are also referred to as biological markers or biomarkers. Incorporation of biomarkers in epidemiologic research may reduce misclassification of exposure or resultant disease. And, perhaps most powerful, is its ability to account for variability and effect modification, i.e. the quantification of inter-individual susceptibility differences [Schulte, 1993]. In other words: biomarkers may explain why at similar exposure some get a disease and some not. A biomarker may represent any event in the continuum of events between causal exposure (E) and resultant disease (CD) (see Figure 1.1) [Perera and Weinstein, 1982; Schulte,1989].

Within this "exposure-disease-continuum", biomarkers may be classified as markers of exposure, markers of effect and markers of susceptibility (see Figure 1.1). Exposure markers first became useful tools in "biological monitoring", especially in occupational health surveillance [Grandjean et al, 1994]. This is underscored, considering their overlap in their definitions: "Biological monitoring is the measurement and assessment of (workplace) agents or their metabolites either in tissue, secreta, excreta, or any combination of these to evaluate exposure and health risk compared to an appropriate reference" [Zielhuis and Henderson, 1986]. The further one moves downwards in the framework (see Figure 1.1), the better the exposure markers improve exposure characterization: traditional (occupational) epidemiology estimates usually can be viewed as (categories of) presumed exposure, while biological markers of exposure measured at the target site, are more likely to be considered as true exposure [Schulte, 1991]. In occupational or environmental settings, exposure markers may be useful in disease prevention by generation of limit values [Grandjean et al., 1994]. An effect marker is a "measurable biochemical, physiologic, or other alteration within an organism that, depending on magnitude, can be recognized as an established or potential health impairment or disease" [Schulte, 1991]. Ideal effect markers are "signals" appearing very early in the exposure-disease continuum, i.e. closer to the time of exposure (see Figure 1.1) [Schulte, 1991, Borm, 1994]. A susceptibility marker is an indicator of an inherent or acquired limitation of an organism's ability to respond to the challenge of exposure to a specific xenobiotic substance [National Research Council, 1987]. In a biological framework of disease pathogenesis, factors determining susceptibility modify the effects of the agent of concern [Mauderly 
and Samet, 1991]. Candidate biomarkers may be generated from in vitro studies, animal studies or studies in humans. A major advantage of animal experiments compared to human studies is the restriction of host and ambient factors [Schulte, 1991]. Human approaches include study designs familiar to epidemiologists, including cross-secional (case-control), prospective and retrospective cohort studies and the intervention study [Schulte et al., 1993].

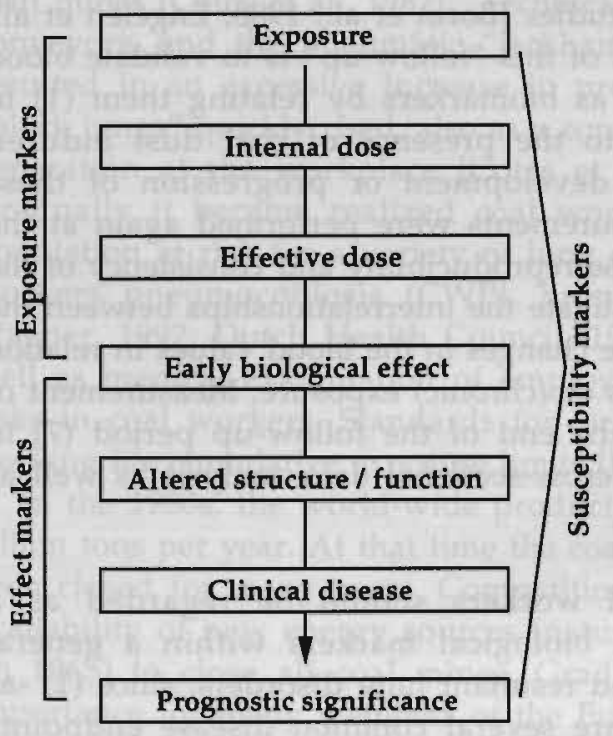

Figure 1.1 Framework of continuum of events in molecular epidemiology

Events between the continuum between causal exposure (E) and resultant disease (CD) can be categorized into (1) the internal dose (ID), i.e. the amount of a xenobiotic or its metabolites found in a biological medium; (2) the effective dose (ED), i.e. the amount of the xenobiotic or its metabolite that interacts with a critical subcellular, cellular or tissue target; (3) the early biological effect (EBE), representing an event correlated with, and possibly predictive of health impairment; and (4) altered structure or function (ASF) which are considered as precursor biological changes that are closely related to disease development [Perera and Weinstein, 1982; Schulte, 1989; Schulte, 1993; Borm 1994]. The resultant disease may be represented by markers that show the presence of current clinical disease (CD), as well as by markers of prognostic significance (PS) to show future development of the disease [Schulte, 1993].

\subsection{Aim of the thesis}

In occupational medicine, there is an increased need to focus on disease prevention, as well as to develop new approaches to study and identify susceptible populations [Cullen et al., 1990; Schulte 1991; Rall and Fouts, 1992; Weill, 1993]. In the past, traditional occupational epidemiology studies have elucidated many health risks [Schulte, 1993], including lung disorders related to high exposure to mineral dusts. Since the time that workplace exposure limits have been introduced, measurements of "physiologic, cellular, subcellular and 
molecular events" in occupational settings have become more important, fortunated by newly developed and improved laboratory methods [Schulte et al., 1993]. Candidate biological markers have often been studied in cross-sectional occupational studies. Unfortunately however, follow-up of these study groups is rare, and thus the extent to which biomarkers predict disease or indicate prognosis often has not been evaluated.

The leitmotiv of this thesis is the recruitment of three groups of coal workers, previously involved in cross-sectional studies [Borm et al., 1988; Engelen et al., 1990; Janssen et al., 1992a]. Primary aim of this "follow-up" is to validate blood parameters measured in these studies as biomarkers by relating them (1) to (cumulative) coal dust exposure, (2) to the presence of coal dust induced disorders, and (3) -prospectively- to development or progression of these disorders. Furthermore, the same measurements were performed again at the end of the follow-up period (4) to analyse reproducibility and consistency of the original cross-sectional data, (5) to investigate the interrelationships between the parameters measured, and (6) to evaluate changes in the blood values in relation to longitudinal changes in disease status or (chronic) exposure. Measurement of new parameters was implemented at the end of the follow-up period (7) to forward new candidate biomarkers by cross-sectional data analysis as well as retrospective analysis of the study group.

In view of this thesis' subject, coal workers should be regarded as a representative population to evaluate biological markers within a general framework of mineral dust exposure and resultant lung disorders, since (1) -as stated before- many mineral dusts share several common disease endpoints including fibrogenesis (pneumoconiosis, PMF) and obstructive lung disease [Parkes, 1982; Rom et al., 1987; Becklake, 1992; Oxman et al., 1993], and (2) in vitro as well as in vivo experiments have yielded considerable similarities in the nature of biological responses to mineral dusts. Therefore, in addition to the generation of a set of (new) biomarkers in mineral dust induced lung disorders, this thesis aims on elucidation of new insights in biological mechanisms of mineral dust induced lung disorders in humans.

In the following chapters, the "classical epidemiology" of coal dust induced lung disorders (chapter 2) as well as their underlying biological mechanisms (chapter 3) are outlined to explain the particular choice of the markers measured in the studies described in this thesis. A short description of the study population, the study design, specific research questions and related selection of the markers are outlined in chapter 4 . 


\section{Chapter 2}

\section{COAL DUST INDUCED LUNG DISORDERS: EPIDEMIOLOGY}

\subsection{Historical background}

Among the various naturally occurring minerals, coal has been recognized as fuel since the Middle Ages, and during the industrial revolution it became the major energy source. Originally, coal was extracted from outcrops and shallow drift mines [Cotes et al., 1987]. Technical inventions such as mechanical pumps, conveyors, and the pneumatic "jackhammer" allowed deep shaft mining and resulted in an excessive increase in production. Incidence and prevalence of "black lungs" quickly rised, also as a consequence of increased (mechanical) dust generation at the workplace [Cotes et al., 1987; Attfield and Wagner 1992]. Gradually it became realized coal workers comprised a major occupational population at risk for a variety of lung diseases [Fletcher, 1948] including Coal workers pneumoconiosis (CWP), bronchitis and emphysema [Attfield and Wagner, 1992; Dutch Health Council, 1992]. Workplace dust measurements as well as medical examination of employees were introduced to control health risks in coal workers. Standards for airborne dust were introduced to ensure working life cumulative exposure limits [Jacobsen et al., 1970].

In the 1980s, the world-wide production of coal was roughly estimated at 3 billion tons per year. At that time the coal mines in the Netherlands had already been closed for many years. Competition with other mining countries and the availability of new energy sources (natural gas) moved the Dutch Government (in 1965) to close all coal mines. Gradually coal mining became of a lesser importance for many members of the European Union, including Germany, the UK, and France. The last Belgian coal mine, situated at Zolder, recently (1992) closed. In sharp contrast, "developing" countries such as China and former Eastern bloc countries remain dependent on coal, but also in the USA coal will remain a major source of energy for considerable time as well owing to increased scarcity and cost of petroleum [Attfield and Wagner, 1992; Bowden, 1994].

In Western Europe, incidence of CWP and silicosis has increased from the beginning of this century until the fifties [Parkes, 1982]. Since that time a steady decrease in number of subjects with silicosis or CWP is observed, which has been attributed to a reduction in number of exposed subjects, improvements in occupational surveillance and to reduced exposure levels and duration [Parkes, 1982; Cullen et al., 1990]. However, from epidemiological studies in retired workers, as well as from morbidity and mortality data in the general population, a rising trend in incidence and prevalence of CWP is now observed in retired workers [Soutar and Hurley, 1986; François et al., 1988; Mahieu, 1990]. Bearing in mind the long latency period for mineral dust induced lung disease, entitlement to earlier and improved retirement benefits in general, as well as increased 
possibilities for a mildly diseased miner to quit or change his job (causing a "healthy worker effect"), both are likely explanations for these observations [Attfield and Wagner, 1992]. Exposure reduction measures may also have caused some drift in the latency period of coal dust induced lung disorders. Evidently in the case of Western Europe, the drastic reduction in mining companies has played a dominant role. Epidemiological studies in the UK demonstrated a higher prevalence of CWP in retired miners versus active workers [Soutar and Hurley, 1986; Soutar 1987]. At the end of 1986 in France, a total of 40,177 men was on the compensation register, while only $3 \%$ of them were still active [Gautrin et al., 1994]. In Belgium 33,325 coal miners received compensation in the year 1991, while at this time most of the mines were already closed. These changes are of major concern in view of the knowledge that -although some handbooks previously stated otherwise- progression of coal workers disease may continue in spite of retirement, i.e. after the cessation of occupational exposure [Shennan et al., 1981; MacLaren and Soutar, 1985]. In France, among the first countries that introduced routine post-occupational surveillance (in 1980 [Gautrin et al., 1994]), "black lungs" have been designated as post-retirement disease for a considerable time [Mahieu, 1990]. While the situation has improved in Western Countries, it is should be realized that due to rapid "industrial revolutions", developing countries are now likely to be confronted with the major occupational health problems that Western countries had to cope with decades ago. Epidemiological studies in China, Brazil, Chili, Columbia, India, Korea and Zimbabwe, recently underscored that coal dust induced lung disorders should be regarded as an important health issue at present and in the future in these countries [Van Sprundel, 1990]. Besides, it is should be realized that health risks from coal dust exposure are not strictly limited to underground mining activities, but also occur in surface mining, processing of coal, and exposure to other carbon compounds including graphite and carbon black [Hanoa, 1983].

\subsection{Coal dust}

Although coal mining activities may result in exposure to many toxic compounds, such as nitrogen oxides, carbon dioxide, radon and other gases, respiratory hazards in coal miners are considered to be almost exclusively a consequence of exposure to dust that has become airborne during extraction or processing of coal [Cotes et al., 1987]. The predominant component of coal is carbon, formed by decomposition and compression of forest trees and related vegetation during the Carboniferous period, some 250 million years ago. Apart from its occurrence as coal, elemental carbon may appear in other forms such as graphite (i.e. crystalline carbon), carbon fibers and carbon black which is a product of partial oxidation or thermal decomposition of hydrocarbons [Cotes et al., 1987]. 
Carbon or graphite fibers are filamentary forms of carbon produced by high temperature processing of organic precursors as rayon, (coal tar) pitch or polyacrylonitrile [Vu, 1994].

Coal extraction exposes miners to a variety of minerals from surrounding layers which may become airborne during extraction of the coal, as well as to other mineral components of the coal dust particles that have become intermingled with the carbon due to earth crust movements. Coal dust may contain quartz, clay, shale, limestone form skeletons and shells of aquatic animals, and other chemical compounds such as iron and calcium. In Europe, the mineral fraction of coal mine dust (i.e. ash content) contains generally 5 to $50 \%$ mica, 4 to $30 \%$ kaolin and 2 to $20 \%$ quartz [Préat, 1993]. Traditionally, coal dust induced lung disorders have been studied in relation to specific composition of dust generated during specific jobs or in specific workplace environment. As an example, exposure to (free) silica may be relatively higher in stone roadways compared to the dust at the coal faces where coal is extracted. While high exposure to free silica may result in silicosis, the relation between quartz content in coal dust and CWP remains obscure, since both positive and negative associations with quartz content and pneumoconiotic risk have been reported [Préat, 1993]. Adverse effects of coal dust are also related to coal rank, which denominates the purity i.e. the quality of the coal as energy source. The high prevalence of CWP associated with high rank anthracyte $(92-94 \%$ pure carbon) compared to bituminous coal $(<92 \%)$ has been attributed to the higher amount of respirable dust generated during mining, as well as to the slower clearance rate from the lung of the former [Cotes et al., 1987]. Thus, many of the described dust properties have been considered to explain for differences in disease prevalence and incidence among mining regions [Gautrin et al., 1994] or among horizontals, or specific jobs within a single mine [Reisner and Robock, 1976]. In this regard, differences in coal extraction and processing techniques among various regions or countries should not be neglected [Préat, 1993].

\subsection{Coal workers pneumoconiosis}

The major respiratory heath effect caused by chronic coal dust exposure is CWP, characterized by a relative mild interstitial inflammation and fibrosis at early stage disease. Although pneumoconiosis in coal workers originally was attributed to silica only, gradually it became accepted that coal played an important role. In 1928, Collis and Gilchrist demonstrated that pneumoconiosis among coal trimmers arose from exposure to coal dust and not only from silica exposure [Collis and Gilchrist, 1928]. In spite of its name, CWP is not strictly limited to coal workers, since it may occur in workers exposed to any carbon dust: Exposure to graphite has been shown to result in disease almost 
indistinguishable from CWP [Gaensler et al., 1966; Okutani et al., 1963], and progressive massive fibrosis (PMF) has been reported in carbon electrode workers [Watson et al., 1959]. Resembling disease has also been observed in oil shale workers [Seaton et al., 1981a]. In CWP, pathology is limited to the lower part of the respiratory tract, and primarily the respiratory bronchioles and alveolar walls are involved [Rom and Crystal, 1991]. Simple CWP is characterized by the formation of macules (or maculae), which are foci of macrophages loaded with dust accumulated in and around the respiratory bronchioles [Cotes et al., 1987, Flint, 1988]. These macules are scattered over the entire lung, but predominantly in the upper lobuli of the lung [Flint, 1988]. At more pronounced disease stage, macules become increased in size, and are also found in the pulmonary arterioles and venes. In the regions of the respiratory bronchioles reticuline and collagen becomes deposited [Flint, 1988; Snyder, 1988].

Development of progressive massive fibrosis (PMF) is due to progressive enlargement of a single coal macule, or by enlargement and fusion of adjacent nodular lesions [Cotes et al., 1987]. A typical PMF biopsy lesion appears as a black mass from which nearly all structural features have disappeared. The mass often engulfs bronchi and pulmonary arteries and extends across septa or from one lobe to another. In PMF, occasionally hypertrophy of the right ventricle and evidence in the body of right ventricular overload is observed [Cotes, 1987]. Sometimes CWP is accompanied with rheumatoid arthritis, known as Caplan's Syndrome [Cotes et al., 1987; Flint, 1988]. Ultimately, death may result from insufficient gas exchange, right-sided heart failure, or overwhelming tuberculosis or infections [Cotes et al., 1987].

A reduced life expectancy of coal workers with progressive massive fibrosis (PMF) was already reported several decades ago [Cochrane 1973]. Support for an increased mortality in simple pneumoconiosis as well as in the absence of CWP was provided by Miller and Jacobson, who additionally showed a correlation with mortality and exposure [Miller and Jacobson, 1985]. Several studies have focussed on the relation between (cumulative) exposure and pneumoconiotic lesions. Exposure assessments have been included in some of the epidemiological surveys in miners allowing to study the concept of a exposure response relationship in coal dust induced lung disorders. In spite of some limitations (e.g. dust measurement bias), these studies have lead to an indication of a general relationship between mass concentration of respirable dust and the incidence of CWP [Cotes et al., 1987], and have been extrapolated to dust control standards (usually in a range of 2 to $4 \mathrm{mg} / \mathrm{m} 3$ ) [Préat, 1993]. Other studies included dust measurements, as well as specific exposure characterization (e.g. coal rank, quartz content, frequencies of high peak-exposures) to explain for regional prevalence differences [Gautrin et al., 1994] development of PMF [Hurley et al., 1987], or rapid developing silicosis [Seaton et al., 1981b]. 


\subsection{Chest radiography examination of pneumoconiosis}

Traditionally, clinical diagnosis of CWP (and other pneumoconioses) is predominantly based on chest radiography. Symptoms as cough and pleghm (i.e. dark sputum) are generally considered as nonspecific symptoms of CWP, since these are also observed in coal dust related chronic bronchitis [Davis and Calhoun, 1988]. As it was realized that this should be optimally standardized, the International Labour Office (ILO) has provided a (regularly revised) classification protocol for chest radiographs. The most recent version dates from 1980 [Parmeggiani, 1983]. It is applied for medical surveillance, in clinical evaluation, and (mainly) in epidemiologic studies. The classification is based on number and size of opacities, and, as indicated before, should be regarded together with a good occupational history to avoid classification error. Other diseases such as bronchiolar carcinoma or sarcoidosis may mimic the rounded opacities of pneumoconiosis [Merchant and Schwartz, 1992]. Based on the number of small opacities in the lung three categories exist i.e. 1, 2 and 3; absence of opacities is classified in category 0 . Each of these categories can be further subdivided into three subcategories, resulting in a total of 12 categories: $-/ 0,0 / 0,0 / 1,1 / 0,1 / 1,1 / 2$, $2 / 1,2 / 2,2 / 3,3 / 2,3 / 3,3 /+$. Each chest radiograph is classified into a main category, which is followed by the classification which is also considered. Size and shape are classified as $\mathrm{p}, \mathrm{q}$, or $\mathrm{r}$ (round) or $\mathrm{s}, \mathrm{t}$, or $\mathrm{u}$ (irregular), based on their size being smaller than $1.5 \mathrm{~mm}$, between 1.2 and $3 \mathrm{~mm}$, or between 3 and $10 \mathrm{~mm}$ in diameter respectively. Irregularly shaped opacities are mainly observed in asbestosis, while rounded opacities are merely characteristic for CWP or silicosis. A chest radiograph is classified into progressive massive fibrosis (PMF) if the size of the opacities exceed a diameter of $10 \mathrm{~mm}$. If the diameter of a single lesion (or the sum of diameters of multiple lesions) ranges form 1 to $5 \mathrm{~cm}$, the radiograph should be classified as $A$. Lesions with a diameter exceeding $5 \mathrm{~cm}$, which have a total area smaller than $1 / 3$ of the right lung are classified as $\mathrm{B}$. If the area of the lesions exceed this size, a chest radiograph is denominated into category $C$.

Limitations of classification of pneumoconiosis have been continuously discussed for a considerable time [Epler et al., 1978]. Major problems in the classification are (1) the assessment of progression of pneumoconiosis, and (2) inter- and intra-reader variability. The latter appears to be especially high at low profusion scores [Begin et al., 1991]. Computed tomography has been applied recently in the clinical evaluation of pneumoconioses, giving rise to increased sensitivity in radiological identification at early stages [Bégin et al., 1991; Bégin et al., 1993; Remi-Jardin et al., 1990; Lamers et al., 1994]. Determination of progression from chest radiographs can be obtained by independent interpretation or, more preferably, by side-by-side readings [Liddell, 1978]. 


\subsection{Nonpneumoconiotic effects of coal dust}

In addition to the development of pneumoconiosis several other lung diseases have been attributed to chronic inhalation of coal (and other) dusts. Most of these findings are provided by occupational epidemiology, or by biopsy/necropsy analysis of lungs of exposed individuals. Major pitfalls in epidemiological studies among coal workers are the attribution of smoking to respiratory effects, the healthy worker effect, and the loss to follow-up of miners quitting their job due to retirement or due to disease.

While evaluation of pneumoconiosis is a matter of radiographic diagnosis, nonpneumoconiotic effects are studied by means of a variety of methods, including questionnaires, sputum analysis, spirometry, impedance measurements, and diffusion capacity measurements [Cotes et al., 1987; Wouters et al., 1994; Jorna et al., 1994a]. Lung function measurements have been introduced in routine medical screening of coal workers many decades ago, and many lung function data are now available for epidemiological studies. Airway obstruction in spirometry is determined by FEV1, the amount of air (Liter) that after maximal inspiration can be exhaled in one second. This is expressed in liters or as percentage of the predicted value, obtained by extrapolation from standard measurements [Quanjer, 1983; ATS, 1987]. By inclusion of measurement of the forced vital capacity (FVC), i.e. the maximal exhalable amount of air after maximal inspiration, also the FEV1/FVC ratio (i.e. Tiffeneau value) can be determined. Lung cancer is also diagnosable by chest radiograph, but classification error may occur in the presence pneumoconiosis lesions. Questionnaires that are used in epidemiologic studies as well as in surveillance and clinical screening, may include questions on cough, shortness of breath, wheezing, pleghm, asthmatic attacks, as well as on past and current smoke habits [Becklake, 1992]. High resolution computed tomography has been implemented in the diagnosis of emphysema [Becklake et al., 1992; Wouters et al., 1994]. As mentioned before, occupational history is important in the case of pneumoconiotic abnormalities, however it should also be stressed that exposure estimates are important in nonpneumoconiotic symptoms.

Inverse relations between lung function and cumulative exposure have been found in several epidemiological surveys in coal miners as well as in other occupations [Kaufmann et al., 1982]. In a cross-sectional survey in British coal workers in 1973 an inverse relation between cumulative respirable dust exposure and FEV1 was found, independent of the presence of CWP [Rogan et al., 1973]. This relation was later confirmed in longitudinal surveys in coal workers in the UK [Love and Miller, 1982] and in the USA [Attfield, 1985], and also showed the attribution of age, length and smoking on FEV1 decline [Marine et al., 1988; Attfield and Hodous, 1992]. Support for the hypothesis that lung function decline due to coal dust exposure may be underestimated due to the healthy worker 
effect was provided by Soutar and Hurley. By inclusion of retired miners in the study cohort, they found that lung function loss was more severe in these subjects, as well as in those who voluntary changed their job, compared to active workers [Soutar and Hurley, 1986]. However, despite this evidence there is still debate as to how far coal dust exposure can lead to severe obstruction in miners without CWP considering the confounding role of smoking [Lapp et al., 1994].

In 1895 Osler concluded that CWP is associated with chronic bronchitis [Wouters et al, 1994]. Chronic bronchitis is a morphologically defined disease, which may be caused by irritation by gases and/or deposition of particulate material on the airways, resulting in hyperplasia of mucus-producing cells lining the airways and an increase in the size and secretory activity of the mucous glands in the walls of the bronchi [Abraham, 1992]. The significant attribution of dust exposure in the prevalence of chronic bronchitis was reported in British coal workers [Rae et al., 1970; Rogan et al., 1973], as well as in non-smoking bituminous coal workers in the USA [Kibelstis et al., 1973]. Soutar and Hurley [1986] found increased bronchitis symptoms (chronic cough, pleghm) in retired workers compared to active workers.

Emphysema is defined by pathologic criteria, as a condition of the lung characterized by abnormal, permanent enlargement of the air spaces distal to the terminal bronchiole accompanied by destruction of their walls [Fletcher and Pride, 1984; ATS 1987]. In coal workers, emphysema is believed to be a result of weakening and dilatation of the bronchiole wall caused by accumulation of dustloaded macrophages in first- and second-order respiratory bronchioles, enmeshed by reticulin, elastin, and collagen [Morgan and Lapp, 1976; Rom, 1990]. The association between coal dust exposure and lung emphysema was already reported by Cummins in the year 1936, based on post-mortem examination of pneumoconiotic lungs [Cummins, 1936]. Later, this relationship was further underscored by others [Lyons et al., 1981; Cockcroft et al. 1982; Ruckley et al., 1984].

The role of coal dust exposure and lung cancer remains a controversial issue [Silicosis and silicate disease committee, 1988] as the confounding effect of smoking is especially important in this relation. While the body of evidence for asbestos as a carcinogen has been increased tremendously over the last decades, the relation between lung cancer and exposure to silica as well as (silica containing) coal dust is not confirmed [McDonald, 1989], although crystalline silica has been classified as a suspect (bronchogenic) carcinogen by a working group of the International Agency for Research on Cancer [IARC, 1987]. It was concluded that there was sufficient evidence from animal studies, but limited evidence for human carcinogenicity of silica and that confounding factors such as smoke cannot be ruled out [IARC, 1987]. An update of the IARC monogrpahs is due in september 1996. In the Netherlands, silica has been classified as human carcinogen by inhalation very recently by the Dutch expert group [DECOS, 1996]. 
Regarding coal dust, mortality studies in coal workers generally have not yielded increased standardized mortality ratios (SMR) for lung cancer in (former) coal workers [Miller and Jacobsen, 1985; Meijers et al., 1991]. Based on these observations, the Dutch Health Council stated that there is 'no reason to assume that labour in the mines, whether causing CWP or not, induces an increased risk for lung cancer [Dutch Health Council, 1992]. Recently, an increased risk was found with long term exposure to coal dust, in a case-control study among lung cancer patients [Morabia et al., 1992]. Rat inhalation studies have yielded both positive [Martin et al. 1975] and negative [Karagianes et al., 1981] results with regard to lung carcinogenicity. In addition to silica, also coal dust will be evaluated by a IARC working group in 1996. From epidemiological studies a relation between coal dust exposure and cancer in the gastrointestinal tract has also been suggested [Stocks, 1965, Matolo et al., 1972; Miller and Jacobsen, 1985; Meijers edt al., 1991]. Support for a role of pulmonary clearance in the development of gastrointestinal malignancies [Meyer et al., 1980], was recenlty obtained in a mortality study in Dutch coal miners, in which the gastric cancer risk turned out to be highest in those with normal FEV ${ }_{1}$ [Swaen et al., 1995]. 


\section{Chapter 3}

\section{COAL DUST INDUCED LUNG DISORDERS: MECHANISMS}

\subsection{Particles in the lung: the role of phagocytes, epithelial cells, and fibroblasts}

The alveolar macrophage is considered as a key cell in mineral dust induced lung disorders [Heppleston and Styles, 1967; Lugano et al., 1984; Driscoll et al., 1990]. Alveolar macrophages are derived from bone marrow monocyte [Thomas et al., 1976] or partly renewed by proliferation of local macrophages [Bitterman et al., 1984; Spurzem et al., 1987], and have a life-span of several months to years. Dependent on their age, the size of alveolar macrophages ranges from $12 \mu \mathrm{m}$ to $40 \mu \mathrm{m}$ [Crystal, 1991]. The crucial role of alveolar macrophages in mineral dust induced disorders is based on several observations:

(1) Subjects chronically exposed to mineral particles have increased number of alveolar macrophages in the lungs, and these macrophages contain ingested particles [Takemura et al., 1989].

(2) Macrophages have the ability to ingest several inorganic particles in vitro [Takemura et al., 1989] as well as in vivo in the lung [Ziskind et al., 1976; Brody et al., 1982]. Macrophage action is considered to be relatively independent of the fibrogenic potential of the particle [Bowden, 1987].

(3) Phagocytosis has been shown to result in an activation state of the macrophage, and during its activation a wide range of products including oxidants, bioactive lipids, cytokines, growth factors, proteases and antiproteases may be released.

Several lung cells may act as targets for these mediators including fibroblasts, epithelial cells and endothelial cells, and all these cell types are capable of releasing secondary mediators. Epithelial cells may also act as direct targets for some of the inhaled particles, resulting in (ir)reversible injury of the epithelium. With regard to mineral dust exposure related bronchitis, bronchial epithelial cells may thus be irritated. This may cause hyperplasia of mucus producing cells and glands in the walls of the bronchi leading to increased mucus production, as well as impaired function of cilliated cells resulting in impaired mucocilliar clearance [Abraham, 1992]. In addition, epithelial cells and (in the case of asbestos fibers) mesothelial cells, may act as target cells in lung carcinogenesis including bronchial carcinoma and mesothelioma [Mossman et al., 1990]. Additional inflammatory cells (e.g. neutrophils) may be recruited from the peripheral blood by factors released by alveolar macrophages as well as by other lung cells. Recruitment of neutrophils is considered to be independent of the fibrogenicity of the particle and will lead to increased inflammatory state in the alveolar region, known as alveolitis [Bowden, 1987; Driscoll et al., 1990]. Thus, in addition 
to direct damage by particles, alveolar epithelial cells may be damaged by inflammatory mediators. This results in the destruction and/or remodeling of the alveolar epithelium: Oxygen uptake may be impaired due to loss of functional alveoalar type I cells by proliferating type II cells of by fibroblasts resulting in alveolar fibrosis [Chrystal et al., 1991]. Degeneration of air space walls may also cause emphysema [Abraham, 1992]. Regarding pulmonary fibrosis, interstitial fibroblasts are considered as key cells [Murray and Laurent, 1988]. This may also be the case in coal (and other mineral) dust induced fibrosis, underscored by the following observations:

(1) Lung fibroblast are a major source of collagen and other interstitial matrix components [Hance et al., 1976].

(2) Development of fibrosis is characterized by the replacement of normal pulmonary parenchyme with a collagen matrix [Snyder 1988, Murray and Laurent, 1988].

(3) Activated macrophages release several factors that have been shown to stimulate fibroblast chemotaxis, growth, proliferation and/or its collagen production. This concept has been originally proposed by Heppleston and Styles [1967], based on the observation that soluble factors released by silicadamaged macrophages were able to stimulate collagen production by cultured fibroblasts.

Although many interactions have been described to date, mechanisms of mineral dust induced lung disorders can be arbitrarily subdivided into two major pathways, involving (1) the production of reactive oxygen species and (2) the release of cytokines and growth factors. Both pathways are based on the concepts of macrophage activation and inflammation, and are considered to lead to diverse respiratory effects that are observed in chronic exposure to mineral dust. Formation of reactive oxygen species may additionally be mediated by intrinsic properties of inhaled mineral dusts, i.e. by non-cellular mechanisms. These biological pathways have been applied to create an exposure disease continuum of coal dust exposure and related diseases that has been described on the previous paragraphs (see Figure 3.1). Since both reactive oxygen species and cytokines are involved in lung diseases other than pneumoconiosis, this review will not be strictly limited to pneumoconiotic pathways of coal dusts. On the other hand, many of the in vitro and in vivo studies have been carried out with mineral dusts other than coal dust, particularly silica and asbestos. Considering the similarities in the mechanisms of mineral dust toxicity, they are included. 

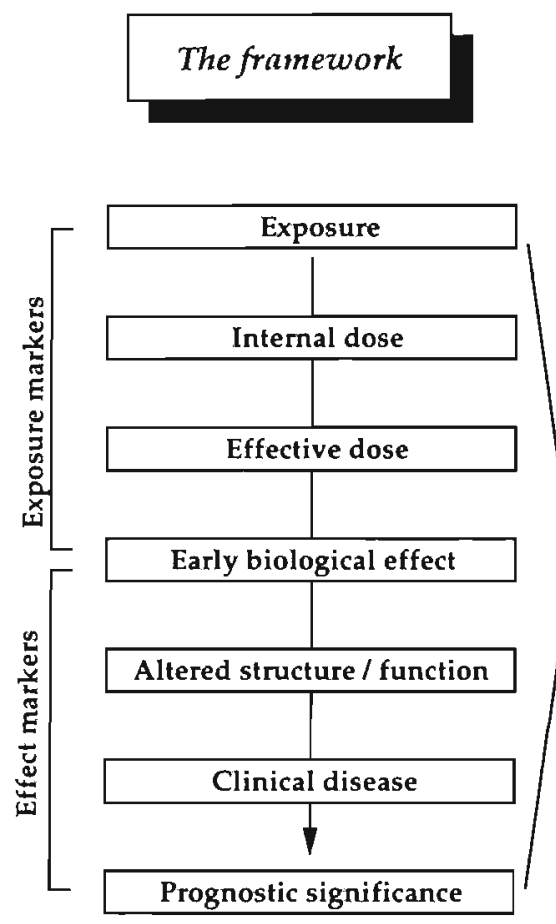

The biological background

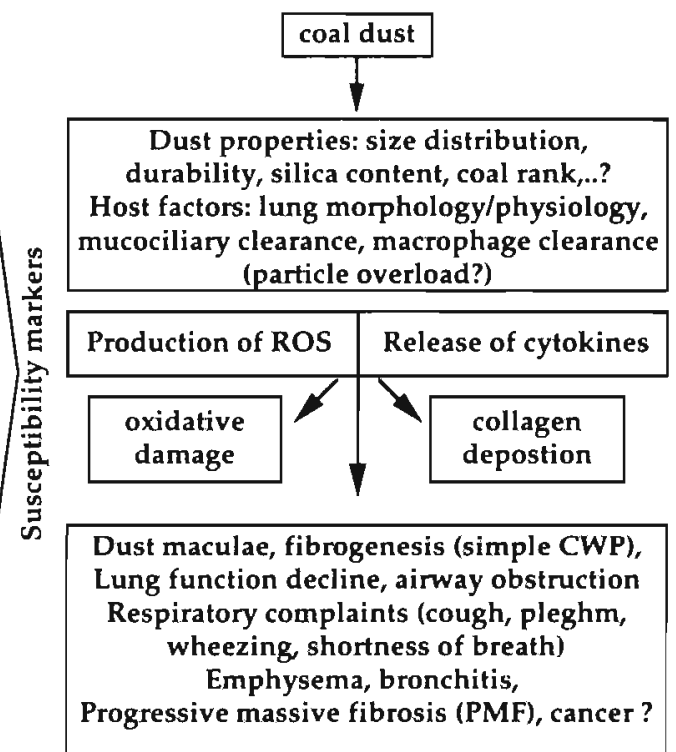

Figure 3.1 Framework of continuum of events related to mineral dust exposure and resultant disease. Within the exposure disease continuum, several events may be distinguished: Exposure to mineral dust (e.g. coal dust) may lead to inhalation of particles. Factors that determine the amount of dust finally reaching the lower part of the respiratory tract (internal dose) are related to the dust (e.g. particle shape ands size), to the environment in which the exposure takes place (e.g. years of employment, occupational hygiene), as well as to the exposed individual (e.g. nose versus mouth breathing, tidal volume). Also the effective dose is related to dust (durability) and host (e.g. cough, mucocilliar clearance, macrophage clearance). Early biological effects may include altered clearance (irritation of airways), and inflammatory responses may occur resulting in altered cellular defense mechanisms (phagocytosis. neutrophil recruitment). Particle overload may cause persistent inflammation. Particles themselves (e.g. particle shape, surface radicals), as well as cell mediated formation of reactive oxygen species, and release of cytokines and other mediators may have effects on surrounding cells and tissue. This may result in (ir)reversible cell- and tissue damage or proliferation (oxidative damage, fibrogenesis), eventually leading to altered lung structure and/or function. Clinical diagnosis may include shortmess of breath, sputum/pleghm, reversible airway obstruction (bronchitis), and radiologic detection of pneumoconiotic lesions (e.g. formation of dust maculae). Eventually, disease may progress into more severe disorders (e.g. chronic bronchitis, severe lung function loss, emphysema, massive fibrosis, lung cancer). 


\subsection{Oxygen damage and antioxidant defense}

\subsubsection{Reactive oxygen species and antioxidants}

Reactive oxygen (ROS) species are implicated in a variety of diseases. The most important cellular effects of ROS are DNA damage, lipid peroxidation and oxidation of proteins (see paragraph 3.2.3). Reactive oxygen species include superoxide anion $\left(\mathrm{O}_{2}{ }^{\circ}\right)$ and the hydroxyl radical $\left(\mathrm{OH}^{\bullet}\right)$, as well as some "reactive" oxygen derivates that do not contain unpaired electrons, such as hydrogen peroxide $\left(\mathrm{H}_{2} \mathrm{O}_{2}\right)$, singlet oxygen $\left({ }^{1} \mathrm{O}_{2}\right)$ and hypochlorous acid $(\mathrm{HOCl})$ (see Table 3.1). In addition, also carbon-centered radicals (e.g. $\mathrm{CCl}_{3}{ }^{\circ}$ ), nitrogen radicals $\left(\mathrm{NO}^{\bullet}, \mathrm{NO}_{2}{ }^{\circ}\right.$ ), and sulfur centered thiylradicals ( $\mathrm{RS}^{\bullet}$ ) are believed to be involved in human disease [Halliwell and Cross, 1994; Kehrer, 1993]. Exogenous compounds that are known to cause production of ROS include ozone $\left(\mathrm{O}_{3}\right)$, nitrogen oxides, bleomycin, paraquat, and radiation.

Cellular sources of ROS comprise various soluble and membrane-bound enzymes, including cytochromes $\mathrm{P} 450$ and b5, the mitochondrial electron transport system, and the cyclooygenase pathway. In 1973 Babior described that activation of phagocytotic cells leads to generation of $\mathrm{O}_{2}{ }^{\bullet-}$ [Babior et al., 1973]. In the lung, primarily macrophages and neutrophils are involved in this "respiratory burst", which utilizes plasma membrane bound NADPH oxidase. Upon activation of this enzyme by (opsonized) particulate stimuli via phagocytosis or by soluble stimuli such as phorbol esters, electrons are shuttled from cytosolic NADPH to oxygen to produce a molecule of $\mathrm{O}_{2}{ }^{\bullet-}$. Neutrophils additionally contain large amounts of myeloperoxidase which converts $\mathrm{H}_{2} \mathrm{O}_{2}$ into hypochlorous acid $(\mathrm{HOCl})$. The highly reactive $\mathrm{HOCl}$ is readily converted to chlorine or can react with primary or secondary amines to form relatively longlived chloramines [Klebanoff et al., 1968; Klebanoff et al., 1980]. Phagocytotic cells are also known to produce nitric oxide ( $\mathrm{NO}^{\bullet}$ ) radicals [Halliwell and Cross, 1994]. $\mathrm{NO}^{*}$ is formed during the oxidation of the amino-acid L-arginine. $\mathrm{NO}^{\bullet}$ may react with oxygen to the $\mathrm{NO}_{2}{ }^{\bullet}$ radical, or with superoxide anion to peroxynitrite $\left(\mathrm{ONOO}^{-}\right)$which may be directly toxic to cells, or in turn lead to the formation of $\mathrm{OH}^{*}$ and related radical compounds [Beckman et al., 1990]. Xanthine oxidase, which is found in peroxysomes, generates $\mathrm{O}_{2}{ }^{--}$and $\mathrm{H}_{2} \mathrm{O}_{2}$ during the conversion of xanthine or hypoxanthine to uric acid [Schraufstätter and Cochrane, 1991].

$\mathrm{O}_{2}{ }^{\bullet-}$ dismutates enzymatically by the superoxide dismutase enzymes (see next session) or spontaneously [Fridovich, 1974]. In the latter case, in addition to $\mathrm{H}_{2} \mathrm{O}_{2}$ also the highly reactive singlet oxygen $\left({ }^{1} \mathrm{O}_{2}\right)$ is formed. $\mathrm{O}_{2}{ }^{--}$and $\mathrm{H}_{2} \mathrm{O}_{2}$ are involved in the formation of the hydroxyl radical $\left(\mathrm{OH}^{\bullet}\right)$, which is the most highly reactive oxygen radical known [Halliwell and Cross, 1994]. This redox reaction occurs in the presence of transition metal ions such as iron and copper 
salts. This iron catalysed reaction is known as the Haber-Weiss reaction [Haber and Weiss, 1934]:

$\mathrm{O}_{2}^{--}+\mathrm{Fe}^{3+} \rightarrow \mathrm{Fe}^{2+}+\mathrm{O}_{2}$
$\mathrm{Fe}^{2+}+\mathrm{H}_{2} \mathrm{O}_{2} \cdots \mathrm{Fe}^{3+}+\mathrm{OH}^{-}+\mathrm{OH}^{\bullet}$

Mammals possess enzymatic antioxidant mechanisms as well as nonenzymatic antioxidants to cope with the toxic effects of reactive oxygen species. Enzymatic antioxidants are the superoxide dismutates (SOD), catalases and the glutathione peroxidases. Nonenzymatic antioxidants include the vitamin A precursor $\beta$ carotene, glutathione, ascorbic acid (Vitamin C), uric acid, $\alpha$-tocopherol (Vitamin E), transferrin, ceruloplasmin, lactoferrin, albumin and taurine (see Table 3.1).

Table 3.1 Reactive (oxygen) species and antioxidants

\begin{tabular}{lll} 
Reactive (oxidant) species & Important antioxidants & Comment \\
\hline $\mathrm{O}_{2}{ }^{*-}$ (superoxide anion) & $\begin{array}{l}\text { Superoxide dismutases (SOD) } \\
\text { Glutathione (GSH) } \\
\text { Vitamins A, C, and E }\end{array}$ & $\begin{array}{l}\text { formation of } \mathrm{H}_{2} \mathrm{O}_{2} \\
\text { formation of } \mathrm{GS}^{\bullet} \text { and } \mathrm{H}_{2} \mathrm{O}_{2}\end{array}$ \\
$\mathrm{OH}^{\bullet}$ (hydroxyl radical) & Vitamin C & formation of Vitamin $\mathrm{C}^{\bullet}$ \\
$\mathrm{H}_{2} \mathrm{O}_{2}$ (hydrogen peroxide) & $\begin{array}{l}\text { Catalase } \\
\text { Se-dependent glutathione } \\
\text { peroxidase (Gpx)\# }\end{array}$ & formation of $\mathrm{H}_{2} \mathrm{O}$ \\
$1_{\mathrm{O}_{2} \text { (singlet oxygen) }}$ & Vitamins A, and E, uric acid & formation of $\mathrm{H}_{2} \mathrm{O}$ \\
$\mathrm{LOOH}$ (lipidhydroperoxides) & $\begin{array}{l}\text { Gpx\#, } \\
\text { Glutathione-S-transferase (GST)\# }\end{array}$ & $\begin{array}{l}\text { Termination of lipid } \\
\text { peroxidation }\end{array}$ \\
$\mathrm{HOCl}$ (hypochlorous acid) & Taurine &
\end{tabular}

- indicates a free radical; \# GSH is required as substrate

\section{Enzymatic antioxidants}

Eukaryotic cells contain two forms of superoxide dismutase (SOD). The copperzinc containing enzyme (CuZnSOD) is found in the cytoplasm [Crapo et al., 1992], in peroxysomes [Keller et al., 1991] as well as extracellularly [Marklund 1984]. Manganese SOD (MnSOD) is present in the mitochondria of eukarytotic cells.

The superoxide dismutase enzymes convert $\mathrm{O}_{2}{ }^{--}$radicals to hydrogen peroxide. The catalase enzymes are involved in the reduction of hydrogen peroxide to water. Intracellular catalase is located in peroxysomes. Extracellular 
catalase is found in blood plasma [Leff et al., 1993] and epithelial lining fluid [Cantin et al., 1985].

There are two classes of glutathione peroxidase enzymes. The selenium dependent glutathione peroxidase (Gpx) is known to reduce hydrogen peroxide to water, during which reduced glutathione (GSH) becomes oxidized. Oxidized glutathione (GSSG) in turn is reduced to GSH in a NADPH dependent reaction catalysed by the enzyme glutathione reductase (GR). The NADPH is provided by the hexose monophosphate shunt. This glutathione redox cycle reaction occurs in the cytosole. The selenium independent glutathione peroxidase, which is also known as glutathione-S-transferase (GST), is not able to reduce hydrogen peroxide to water. Gpx as well as GST reduce lipid hydroperoxides into lipid alcohols, a reaction which also involves oxidation of GSH to GSSG. In addition to its scavenging role in lipid peroxidation, GST plays a role in the detoxification of electrophilic compounds in GSH conjugation reactions. GST is found in cytosole and microsomes. Human cytosolic GST can be divided into class A, M, P and $T(\alpha, \mu, \pi$ and $\vartheta)$. Polymorphism in humans has been described for GSTM [Seidegard et al., 1986].

\section{Nonenzymatic antioxidants}

In addition to its role as cosubstrate in the glutathione redox cycle, glutathione may also act as a direct antioxidant. Scavenging of superoxide anion by glutathione leads to the formation of a sulfur-centered thiyl radical (GS ${ }^{\bullet}$ ) and hydrogen peroxide. Furthermore, glutathione is known to scavenge hydrogen peroxide and other peroxides. Glutathione is found intracellularly, as well as extracellularly in the blood plasma and epithelial lining fluid [Cantin et al., 1985]. Because of its abundancy in many biological systems, glutathione is believed to be one of the main detoxificants of xenobiotics. As already mentioned above, the enzyme Glutathione-S-transferase is involved in the conjugation of glutathione, and a reduced activity or availability of Gpx or GST may have consequences for GSH detoxification action.

Together with glutathione, ascorbic acid (Vitamin C) is the most important water soluble antioxidant, and is found both intracellularly and extracellularly. Vitamin $\mathrm{C}$ can scavenge both $\mathrm{O}_{2}{ }^{\circ-}$ and $\mathrm{OH} \bullet$ and the resulting vitamin $\mathrm{C}$ free radical subsequently can be reduced by glutathione. On the other hand vitamin $C$ has prooxidant action e.g. by its ability to convert $\mathrm{Fe}^{3+}$ to $\mathrm{Fe}^{2+}$ thus likely promoting Fenton-type reactions (eq. II).

Vitamin E ( $\alpha$-tocopherol) is the most important lipid soluble antioxidant, and limits membrane injury in human tissue. Vitamin $E$ acts as a chain reaction terminator in the lipid peroxidation process, during which it becomes a radical itself, which however is fairly stable compared to the lipid radicals [Burton and Ingold, 1981]. Furthermore, Vitamin E has antioxidant action for the superoxide radical, and it is known both to quenche and react with for singlet oxygen. 
Other singlet oxygen quenchers include the lipid soluble Vitamin $A$ and uric acid, which is formed by catabolism of purines. Vitamin A also can scavenge $\mathrm{O}_{2}{ }^{--}$ and peroxyl radicals [Foote and Denny, 1968]. Uric acid acts as a scavenger of $\mathrm{OH} \bullet$ and peroxyl radicals, it prevents oxidation of vitamin $C$ and it binds to transition metals [Ames et al., 1981]. Other antioxidants include iron or copper chelators as transferrin [Pacht et al., 1988], ceruloplasmin [Goldstein et al., 1979] and albumin [Brown et al., 1989a; Halliwell, 1988], and the amino-acid taurine which detoxifies $\mathrm{HOCl}$ [Klebanoff et al., 1980].

\subsubsection{Reactive oxygen species and antioxidants in mineral dust induced lung disorders}

Adverse effects of ROS in the lung may include (1) damage to cell membranes by lipid peroxidation, (2) oxidation of proteins, and (3) DNA damage. Lipid peroxidation is a chain reaction process which involves an initial reaction of a free radical with an unsaturated fatty acid of the cellular membrane resulting in the formation of a lipid radical which reacts with oxygen to form a lipidperoxyl radical. This lipidperoxylradical in turn may react with another unsaturated membrane fatty acid resulting in a reactive lipidhydroperoxide molecule and a new lipid radical. The resulting chain reaction may lead to cell damage and tissue remodeling. Reactions of ROS with proteins may lead to inactivation of enzymes involved in cell metabolism or in modification of intra- or extracellular structural components. Oxidative DNA damage may have various consequences ranging from cell death and tissue destruction to cell or tissue proliferation [Janssen et al., 1993]. A role for ROS has been considered in the pathogenesis of several lung diseases, including hyperoxic injury [Chvapil et al., 1975], radiation pneumonitis [Gross et al., 1977], collagen-vascular diseases [Hunninghake et a]., 1978], adult respiratory distress syndrome (ARDS), and idiopathic pulmonary fibrosis [Cantin et al., 1987b]. At present only the role of ROS in mineral dust induced lung disorders will be discussed. Basically, two mechanisms by which mineral dust exposure causes formation of ROS in vivo have been proposed:

(1) Formation of ROS by intrinsic properties of the particles, i.e. non-cellular mechanisms.

(2) Excessive formation of ROS by the oxidative burst of macrophages and neutrophils activated during particle phagocytosis and inflammation.

(1) Non-cellular generation of ROS. Over the last decades several mechanisms have been proposed regarding the noncellular toxicity of mineral particles, including the role of silanol groups on the surface of silica [Nash et al., 1966], surface charge properties related to the crystalline structure of silica and asbestos [Brown et al., 1989b; Fubini, 1993], and the iron content of asbestos fibers [Zalma et al., 1987]. Grinding i.e. cleavage of coal dust (as well as silica) is believed to 
cause generation of radicals on "fresh" surfaces [Vallyathan et al.,1988; Dalal et al., 1989]. The iron present on the surface of asbestos fibers may be involved in the formation of hydroxyl radicals via the Fenton-reaction (eq. II) [Weitzman and Graceffa, 1984; Goodlick and Kane, 1986; Zalma et al., 1987]. Iron content may also play an important role in the toxicity of coal dust [Tourman and Kaufmann, 1994; Dalal et al., 1995]. Fenton-reaction type formation of hydroxyl radicals was found to be positively correlated to iron content of coal dust [Dalal et al., 1995].

(2) Cell-mediated generation of ROS. Indirect generation of radicals is considered to be controlled by the alveolar macrophage. Macrophages incubated with mineral dusts produce excessive amounts of oxygen radicals [Kamp et al., 1992], as well as chemoattractive factors for other inflammatory cells including monocytes and PMN which in turn may produce ROS and amplify local radical formation. Also physical and chemical properties of particles were found to be related to the extent to which ROS are generated from phagocytic cells [Hansen and Mossman, 1987], and very large fibershaped particles can cause extracellular burst by incomplete or "frustrated" phagocytosis by phagocytotic cells [Mossman et al., 1987a]. Further support for increased hydroxyl radical generation in the course of pneumoconiosis disease was recently obtained in an in vivo silica model of fibrosis [Shapira et al., 1995].

Evidence for the involvement of ROS in humans exposed to mineral dust was initially based on observations from bronchoalveolar lavage studies. Alveolar macrophages of mineral dust exposed subjects produced increased amounts of oxygen radicals compared to non-exposed subjects [Voisin et al., 1985; Rom et al., 1987]. Comparison of oxidant generating capacities of alveolar macrophages in simple CWP versus PMF yielded a possible correlation with disease severity [Wallaert et al. 1990]. Electron spin resonance demonstrated -in line with the presence of radicals on coal and silica dusts [Dalal et al., 1989]- increased amounts of radicals in lung biopsies, and more specifically in lymph nodes, of exposed subjects compared to controls [Dalal et al., 1991].

ROS are also considered to play a crucial role in the pathogenesis of emphysema by oxidative damage to antiproteases reducing its activity. Reduced antiprotease activity may cause exaggerated lung tissue destruction by proteases [Carp et al., 1982]. Similarly, it has also been suggested that dust mediated oxidant generation may be involved in the focal emphysema in coal workers [Rom, 1990]. The $\mathrm{FeSO} 4$ content of coal dust (which is formed from oxidation of pyrite/FeS2) has also been suggested to play a dominant role in antiprotease inactivation [Huang et al., 1994].

The role of ROS in carcinogenesis has been studied extensively [Kensler and Trush, 1984; Trush and Kensler, 1991]. Oxidative mechanisms are also likely implicated in malignant effects observed in humans exposed to mineral dusts 
[Mossman et al., 1989; Janssen et al., 1993]. In the DNA damage caused by ROS in vivo, both $\mathrm{O}_{2}{ }^{\bullet-}$ and $\mathrm{H}_{2} \mathrm{O}_{2}$ are most likely not directly involved. However, $\mathrm{OH} \bullet$ has been shown to induce various types of DNA damage, most likely in Fenton reaction related mechanisms [Aruoma et al., 1989]. Since $\mathrm{H}_{2} \mathrm{O}_{2}$ can easily diffuse through cell membranes [Chance et al., 1979], $\mathrm{OH} \cdot$ generation may occur extra- or intracellularly. Because of its reactivity $\mathrm{OH} \bullet$ will always cause damage at the site of its formation [Schraufstätter and Cochrane, 1991]. C8 oxidation of deoxyguanosine $(\mathrm{dG})$, resulting in 7-hydro-8-oxo-2'-deoxyguanosine (8-oxodG) is one of the most prevalent naturally occurring DNA lesions in response to ROS. The ratio 8-oxodG/dG has been forwarded as biomarker of oxidative stress [Kasai et al., 1986; Kuchino et al., 1987], and has been studied in relation to mineral dust exposure in vitro [Leanderson et al. , 1988; Adachi et al., 1994; Takeuchi et al., 1994]. As collection of blood lymphocytes is considered as a relatively noninvasive method to obtain DNA, 8-oxodG/dG ratios in these surrogate cells are considered as relevant markers of oxidative DNA damage in target cells and tissue including the lung. Recently, 8-oxodG/dG was determined in peripheral blood lymphocytes of asbestos workers [Hanaoka et al., 1993]. Silica also causes formation of strand breaks in vitro, likely related to surface properties of these particles [Daniel et al., 1993].

Studies using radical scavengers or antioxidants have underscored the significance of ROS in lung toxicity [Goodglick and Kane, 1986; Mossman et al., 1987b; Voisin et al., 1985; Marsh and Mossman, 1991], including pulmonary fibrosis [Mossman et al., 1990]. Based on these observations, adverse oxidative effects have been suggested to an impaired balance between ROS and antioxidant defense status, and upregulation of antioxidant enzymes is considered as a marker of oxidative stress [Borm et al., 1986; Janssen et al., 1994]. For instance, hydrogen peroxide causes increases expression of catalase, MnSOD and Gpx in vitro [Shull et al, 1991], and both in vitro and in vivo studies showed that mineral dusts induce the expression as well as release of antioxidant enzymes including catalase and MnSOD [Janssen et al., 1992; Janssen et al., 1994]. Red blood cells contain large amounts of antioxidants and are considered to act as powerful antioxidant carriers for oxidative lung toxicity [Toth et al., 1984; van Asbeck et al., 1984]. Consequently, altered levels of red cell antioxidants (GSH, catalase, SOD) found in CWP likely reflect the significance of radical mechanisms in coal dust exposure and pneumoconiosis disease in humans [Borm et al., 1986; Engelen et al., 1988; Evelo et al., 1993]. Recently, altered antioxidant levels were found in bronchoalveolar lavage of coal workers compared to non-exposed subjects [Vallyathan et al., 1995]. The proposed role of reduced vitamin A and E status in pulmonary fibrosis may be related to their importancy as free radical scavenger and chain reaction terminator in the lipid peroxidation process. A reduced nutrient intake of vitamins ( $A, C$ and $E$ ) has also been associated with increased risk of progressive lung function loss and airway obstruction [Morabia 
et al., 1989; Schwartz and Weiss, 1994; Britton et al., 1995], and dietary vitamin A status has been considered as a "susceptibility" determinant in coal workers [Swartz et al., 1978]. Reduced extracellular GSH content (epithelial lining fluid) has been associated to increased lung fibroblast proliferation [Cantin et al., 1990], and a resulting therapeutic role in lung fibrosis for GSH has been proposed. Other antioxidant therapies currently in focus include administration of SOD or catalase-lipids. In line with the possible role of GST-M polymorphism in smoking related lung cancer [Seidegard et al., 1986, 1990], it has been suggested recently, that GST-M1 deficient subjects are at increased risk for asbestosis [Smith et al. 1994]. However, others have failed to show such genetic predisposition [Jakobsson et al., 1994].

\subsection{Cytokines}

\subsubsection{Cytokines and mineral dust}

Cytokines are polypeptides that are produced by and regulate the function of virtually all nucleated cells [Elias and Zitnik, 1992]. Cytokines share several common features. With a few exceptions, all cytokines are multifunctional in a wide spectrum of biological events including inflammation, metabolism, cell growth and differentiation, morphogenesis, fibrogenesis, and/or homeostasis [Elias and Zitnik, 1992]. Cytokine action may be concentration dependent, and is effectuated via binding to specific cell receptors. Cytokines may act autocrine, paracrine and/or heterocrine. Cytokine action is also dependent on the activation state and/or maturation of their target cells as well as the composition and state of degradation of the surrounding matrix [Elias and Zitnik, 1992]. Cytokines function in cascades or cytokine networks, i.e. cytokine effects are dependent on the presence or absence of other cytokines in a local microenvironment [Kelley, 1990]. In addition to cells of the immune system, the major cytokine sources in the lung are epithelial cells, endothelial cells and fibroblasts. This review will mainly focus on the mostly appreciated candidate cytokines in the two-step mechanism by macrophages and fibroblasts involved in pneumoconiosis disease. Table 3.2 shows cytokines as well as some other secretory products that are released by monocytes or macrophages upon in vitro stimulation with coal dust. Since an important role of the (toxic) properties of coal dust has been attributed to its silica content, relevant data obtained with silica in the same as well as other studies are also included. Furthermore, most important findings with asbestos are described.

Several candidate cytokines and other macrophage derived factors have been forwarded (Table 3.3) to control the hallmarks of pulmonary fibrosis, i.e. fibroblast activation, proliferation and production of extracellular matrix components. Many candidates have been forwarded from in vitro studies in cultured fibroblasts and are of limited value to the in vivo situation. More 
Table 3.2 Factors released by monocyte/macrophage upon in vitro incubation with coal dust, asbestos, or silica

\begin{tabular}{|c|c|c|c|c|}
\hline Cell /source & dust & factor & Remarks & References \\
\hline Macrophage/murine & Silica & IL-1 & & Gery et al., 1981 \\
\hline Macrophage/murine & Silica, Asbestos & IL-1 & $\mathrm{TiO} 2$ does not stimulate IL-1 release & Oghiso et al., 1987 \\
\hline Monocyte/human & Silica & IL-1 & & Schmidt et al., 1984 \\
\hline Monocyte/human & Coal, Silica & TNF & & Borm et al., 1988 \\
\hline Macrophage/murine & Silica, Asbestos & TNF & & Bisonette et al., 1989 \\
\hline Macrophage/human & Silica, Asbestos & TNF; LTB4 & & Dubois et al., 1989 \\
\hline Macrophage/murine & Silica, Asbestos & FN & TiO2 does not stimulate FN release & Davies et al., 1989 \\
\hline Macrophage/murine & Silica, Asbestos & TNF; LTB4 & $\begin{array}{l}\text { No elevated IL-1 release; } \\
\text { TiO2 does not stimulate TNF or LTB4 }\end{array}$ & Driscoll et al., 1990 \\
\hline Macrophage/human & Coal, Silica & TNF; IL-6 & $\begin{array}{l}\text { No elevated release of } I L-1 \text {; } \\
\text { silica does not stimulate IL-6 }\end{array}$ & Gosset et al., 1991 \\
\hline Macrophage/murine & Coal, Silica & $\mathrm{PGE}_{2} ; \mathrm{TXA}_{2}$ & $\begin{array}{l}\text { Released by "fresh" coal dust only; } \\
\text { no stimulation of LTB4 }\end{array}$ & Kuhn et al., 1992 \\
\hline Macrophage/human & Coal, Silica & PAF & $\begin{array}{l}\text { Coal dust induced release approx. } \\
20 \% \text { compared to silica }\end{array}$ & Lapp et al., 1993 \\
\hline Macrophage/human & Asbestos & TNF & $\begin{array}{l}\text { No elevated IL-1, IL- } 6, \text { GM-CSF, PGE } 2 \\
\text { TiO2 does not stimulate any factor }\end{array}$ & Perkins et al., 1993 \\
\hline Macrophage/human & Coal, Silica & PDGF; TGF; IGF-I & TiO2 stimulates IGF-I, but not TGF or PDGF & Vanhée et al., 1994 \\
\hline
\end{tabular}


W Table 3.3 Major factors released by alveolar macrophages recognized for their stimulating or inhibitory effects on fibroblasts in vitro

\begin{tabular}{|c|c|c|}
\hline Factor & Effect on fibroblasts & References \\
\hline TNF & $\begin{array}{l}\text { growth }(+) \\
\text { collagen production }(+) \\
\text { collagen gene expression }(+)\end{array}$ & $\begin{array}{l}\text { Vilcek et al., 1987; Leibovich et al., 1987; } \\
\text { Beutler and Cerami, 1987; Martinet et al., } 1988 \\
\text { Elias et al., } 1990\end{array}$ \\
\hline IL-1 & $\begin{array}{l}\text { growth }(+) \\
\text { collagen gene expression }(t)\end{array}$ & $\begin{array}{l}\text { Schmidt et al., 1982; Bitterman et al., } 1986 \\
\text { Dayer et al., 1986; Elias et al., } 1987 \\
\text { Goldring and Krane, } 1987\end{array}$ \\
\hline TGF & $\begin{array}{l}\text { growth }(+) \\
\text { growth }(-) \\
\text { chemoattraction }(+) \\
\text { chemoattraction }(0)\end{array}$ & $\begin{array}{l}\text { Ignotz et al., 1986; Fine and Goldstein, 1987; Wahl et al., } 1989 \\
\text { Spom et al., 1986; Yamauchi et al., 1988; Moses et al., 1990; Raghu et al., } 1 \text { 1499 } \\
\text { Postlethwaite et al., } 1987 \\
\text { Osornio-Vargas et al., } 1993\end{array}$ \\
\hline PDGF & $\begin{array}{l}\text { growth }(+) \text {, chemoattract }(+) \\
\text { collagen gene expression }(+) \\
\text { collagen production }(0)\end{array}$ & $\begin{array}{l}\text { Martinet et al., 1987; Leibovich and Ross, 1976; Shaw et al., 1984 } \\
\text { Narayanan et al., } 1983 \\
\text { Clark et al., } 1993\end{array}$ \\
\hline IGF-1 & $\begin{array}{l}\text { growth(+) } \\
\text { collagen gene expression (+) }\end{array}$ & $\begin{array}{l}\text { Bitterman et al., 1982; Rom et al., } 1988 \\
\text { Goldstein et al., } 1989\end{array}$ \\
\hline IFN & $\begin{array}{l}\text { growth }(+) \\
\text { growth }(-) \\
\text { collagen production }(-) \\
\text { collagen gene expression }(0)\end{array}$ & $\begin{array}{l}\text { Elias et al., } 1987 \\
\text { Hyde et al., } 1988 \\
\text { Amento et al., } 1985 \\
\text { Narayanan et al., } 1989\end{array}$ \\
\hline FN & growth/chemoattraction $(+)$ & Rennard et al., 1981 \\
\hline PGE & growth (-) & Elias et al., 1985 \\
\hline
\end{tabular}


relevant are in vivo studies performed in animals, using mineral dust models of fibrosis (e.g. silica, asbestos, $\mathrm{TiO}_{2}$ ), or bleomycin [Giri et al., 1980; Laurent et al., 1983; Raghow et al., 1985]. Additionally, some studies have used bronchoalveolar lavage cells (alveolar macrophages) or fluid from exposed animal or humans. In this review, mainly the role of the most relevant cytokines with regard to fibrosis will be discussed below. However also some nonpneumoconiotic effects that might be mediated by cytokines are briefly outlined.

\subsubsection{Tumor Necrosis Factor- $\alpha$ and receptors.}

Tumor necrosis factor- $\alpha$ (TNF) is the name given to a $17 \mathrm{kDa}$ protein released by lipopolysaccharide (LPS) stimulated murine macrophages, that caused hemorrhagic necrosis in experimental intradermal tumors in mice [Carswell et al., 1975]. It appeared to be the same protein as cachectin, a endogenous humoral mediator of cachexia in infections [Beutler et al., 1985; Cerami et al., 1985]. TNF is a crucial factor in endotoxin related toxic effects and septic shock can be prevented by TNF antibodies [Tracey et al., 1987]. TNF is predominantly released by macrophages and monocytes, but also other cells produce TNF such as neutrophils [Djeu et al., 1990] and lymphocytes [Sung et al., 1988]. Macrophages release higher amounts of TNF than monocytes [Martinet et al., 1988]. The human gene of TNF is situated on chromosome 6, linked to the gene encoding Tumor Necrosis Factor- $\beta$ (or lymphotoxin), close to the Major Histocompatibility Complex (MHC) region [Schollmeier, 1990]. Important effects of TNF, which are mediated by TNF receptors, include induction of fibroblast growth [Vilcek et al., 1986; Martinet et al., 1988], collagenase and PGE2 release [Dayer et al., 1985], expression of neutrophil adherence molecules [Gamble et al., 1985], enhancement of oxidant production from neutrophils [Klebanoff et al., 1986], macrophages [Warren et al., 1988], and fibroblasts [Meier et al., 1989] and induction of expression of antioxidants [Tsau et al., 1990; Wong and Goeddel., 1988]. TNF is chemotactic for neutrophils and monocytes [Ming et al., 1987]. TNF release may be enhanced by many agents or events including lipopolysaccharides (LPS), and complexes of LPS and LPS-binding protein (LBP), interferon- $\gamma$, IL- 1 , protein kinase-C activating agents, reactive oxygen species [Chaudhri and Clark, 1989], monocyte adherence, C5a, Vitamin D3, granulocyte-macrophage and macrophage colony stimulating factors (GM-CSF and M-CSF), and ionizing radiation. Agents known to suppress TNF release include glucocorticosteroids [Waage et al., 1987] and prostaglandin-E2 [Kunkel et al., 1988].

TNF release by monocytes/macrophages has been observed in response to several mineral dusts (see Table 3.2). Stimulation with coal dust particles results both in an enhanced mRNA expression as well as protein release in a dose response manner [Borm et al., 1988; Gosset et al., 1991]. Gosset and coworkers showed that in comparison to silica, coal dust had an extra effect on macrophage TNF release which was not attributable to its silica content [Gosset et al., 1991]. 
The first in wio evidence of TNF in mineral dust induced fibrosis was indicated hy increased spontaneous and stimulated release of TNF from peripheral blood monocytes of (pneumoconiotic) coal workers compared to controls [Borm et al., 1988]. Baseline as well as (dust) stimulated TNF release by monocytes of coal workers without CWP were increased compared to controls, and was even further increased in those subjects with early radiologic stage defined CWP [Borm et al., 1988]. Similarly, a higher spontaneous TNF release from alveolar macrophages of subjects with PMF compared to subjects with simple pneumoconiosis and non-exposed controls was reported [Lasalle et al., 1990]. Increased release of TNF was also found in macrophages of rats exposed to silica [Driscoll et al., 1990] and in alveolar as well as peritoneal macrophages [Mohr et al., 1991]. Crucial in vivo support for the role of TNF in mineral dust induced fibrosis was provided by experiments of Piguet and coworkers [Piguet et al., 1990]. In mice exposed to silica, total lung TNF mRNA was increased up to 70 days post exposure, and in situ hybridization elucidated increased TNF levels in silicotic nodules [Piguet et al., 1990]. Furthermore, administration of anti-TNF reduced the hydroxyproline content as a measure of fibrosis, while silica plus recombinant TNF (but not recombinant TNF only) increased fibrosis [Piguet et al., 1990]. More recently, in subjects chronically exposed to asbestos without asbestosis elevated baseline macrophage release of TNF (and IL1, IL6 and PGE2) was observed compared to controls [Perkins et al., 1993]. Changes in macrophage subpopulations (i.e. increased recruitment of monocytes) during development of bleomycin induced fibrosis are considered to be responsible for changes TNF release, as TNF release was demonstrated to be specific to a macrophage subset [Everson and Chandler, 1992]. The large individual variation in monocyte/macrophage TNF release is considered to be related to genetic polymorphism [Mölvig et al., 1988; Pociot et al., 1993], and may be closely related to polymorphism described for HLA [Wilson et al., 1992]. HLA related genetic predisposition to mineral induced fibrosis has been suggested for silicosis [Honda et al., 1988] and more recently for CWP [Rihs et al., 1994].

Two distinct transmembrane TNF receptors proteins have been cloned, with molecular weights of $55 \mathrm{kD}$ and $75 \mathrm{kD}$ respectively [Loetscher et al., 1990; Schall et al., 1990; Smith et al., 1990]. Release of both TNF receptor types from cellular membranes, has been described for various cells including neutrophils, lymphocytes and monocytes. The resulting soluble TNF receptors (sTNFr55 and sTNFr75) possibly constitute a powerful feedback mechanism for (systemic) TNF release in vivo. Soluble TNF receptors inhibit acute (LPS induced) inflammation [Ulich et al., 1993] and endotoxemia [Spinas et al., 1992; Shapiro et al., 1993; Bemelmans et al., 1993]. Increased serum levels of sTNFr have been reported in cardiovascular disease [Latini et al., 1994], cirrhotic liver disease [Tilg et al., 1993], and have been forwarded as markers in malignant diseases [Aderka et al., 1991; Naylor et al., 1993]. Recently, increased bronchoalveolar lavage levels of soluble 
TNF receptors were observed in several inflammatory lung diseases including idiopathic pulmonary fibrosis [Walker et al., 1994]. Similar to their previous experiments using TNF antibodies [Piguet et al., 1990], Piguet and coworkers recently demonstrated a preventive as well as curative role of infused TNF receptors in bleomycin or silica induced fibrosis [Piguet and Vesin, 1994]

\subsubsection{Transforming Growth Factor- $\beta$}

Transforming growth factor- $\beta$ proteins are synthesized as large, biologically inactive precursors. At least three types have been described in human, i.e TGF $\beta 1$, TGF 2 and TGF 33 [Kelley, 1993]. TGF 31 and TGF 32 are $25 \mathrm{kDa}$ and $24 \mathrm{kDa}$ proteins, respectively, which share about $70 \%$ homology [Kelley, 1990]. Genes encoding TGF $\beta$ proteins are located on chromosome 19, and major sources include blood platelets, alveolar macrophages [Vanhée et al., 1994], peripheral blood monocytes and neutrophils [Grotendorst et al., 1989]. TGF $\beta$ is secreted in a latent form, but may be activated in vitro by detergents, proteolytic enzymes or brief exposure to extreme pH [Kelley, 1993]. Latent TGF $\beta$ cannot bind to its receptors, and the activation of TGF $\beta$ in vivo is considered as an important regulatory step. In vivo TGF $\beta$ is believed to be cleaved by plasmin or cathepsin G [Lyons et al., 1988]. TGF $\beta$ released by monocytes/macrophages appears to be preactivated as a consequence of proteolytic processing on the cell surface [Grotendorst et al., 1989]. Alveolar macrophages constitutively express TGF $\beta$. [Assoian et al., 1987]. TGF $\beta$ action is mediated via several specific receptors [Wakefield et al., 1987]. TGF $\beta$ is chemotactic for monocytes [Wahl et al., 1987], neutrophils [Reibman et al., 1991], and fibroblast [Postlethwaite et al., 1987]. TGF $\beta$ has been reported to increase the production of collagen [Fine and Goldstein, 1987; Roberts et al., 1986] and fibronectin [Dean et al., 1988; Ignotz et al., 1986], as well as of protease inhibitors while synthesis of proteases is decreased [Kelley, 1993]. TGF $\beta$ has been reported to produce collagen by fibroblast in the absence of proliferation [Raghu et al., 1986]. However, others have found contradictory results of TGF $\beta$ action on fibroblasts (see Table 3.3), including the absence of chemotactic activity towards fibroblasts [Osornio-Vargas et al., 1993]. In vitro response TGF $\beta$ is strongly dependent on cell type, presence of other growth factors, and the activation state of the cells before exposure to TGF $\beta$ [Kelley, 1993]. Based on in vitro observations, it is suggested that the fibrotic action of TGF $\beta$ may dependent on its concentration, i.e. a stimulatory effect on fibroblasts at low concentration and an inhibitory effect at higher concentrations [Battegay et al, 1990].

TGF $\beta$ is also released in vitro by macrophages stimulated with silica or coal dust [Vanhée et al., 1994], and the in vivo action of TGF $\beta$ in fibrosis has been studied in animal models using bleomycin and silica. In the bleomycin model, TGF $\beta$ mRNA expression was considered as an early event that was followed by collagen production [Raghow et al., 1989; Hoyt and Lazo, 1990]. Increased TGF $\beta$ is 
also found in human fibrosis [Khalil et al., 1991], and is simultaneously expressed with fibronectin and type I procollagen [Broekelman et al., 1991]. Interestingly, Piguet and coworkers found increased TGF $\beta$ mRNA in the bleomycin model [Piguet et al., 1989], but not in the silica model, opposite to TNF mRNA [Piguet et al., 1990]. Vanhée and coworkers showed that alveolar macrophage supernatants from simple CWP patients, but not from PMF patients, inhibited fibroblast growth in vitro, and that this "anti-fibrotic" effect could be counteracted by addtion of anti-TGF $\beta$ in the supernatants [Vanhée et al., 1995]. Recently it was demonstrated that TGF may cause increased oxidative damage in alveolar cells by means of depletion of antioxidant enzymes including glutathione, glutathione reductase and catalase [Arsalane et al., 1995].

\subsubsection{Other cytokines relevant to mineral dust induced effects}

Other relevant cytokines that will be discussed in view of possible involvement in the development or progression of mineral dust induced disorders are interleukin-1, interleukin-6 and platelet derived growth factor.

Interleukin-1 (IL-1) is also known as endogenous pyrogen, based on its ability to cause fever in response to infection [Atkins, 1960], or lymphocyte-activating factor (LAF) [Gery et al., 1972]. Interleukin-1 is a $17.5 \mathrm{kDa}$ protein. IL- $1 \alpha$ and IL-1 $\beta$ proteins share $26 \%$ homology, but bind to an identical $80 \mathrm{kDa}$ IL-1 receptor [Sims et al., 1988]. IL-1 is predominantly produced by monocytes and macrophages [Wewers et al., 1989], and IL-1 production is reduced in smokers [Yamaguchi et al., 1989]]. The IL-1 genes are located on chromosome 2. IL-1 is produced by a variety of activated cells, including monocytes and macrophages, B-lymphocytes, Natural Killer cells, neutrophils and fibroblasts [Dinarello and Savage, 1989]. Major sources of IL-1 are stimulated monocytes and macrophages, which predominantly release IL-1 $\beta$. Expression of IL-1 in these cells may be increased by endotoxin, phorbol esters and cytokines including TNF $\alpha$ and IL-1. Monocyte adherence also induces IL-1 release [Fuhlbrigge et al., 1987]. Like TNF, production of IL-1 may be attenuated by prostaglandin E2 and glucocorticosteroids [Aksamit and Hunninghake, 1993]. Recently an IL-1 receptor antagonist has become recognized as an important inhibitor of IL-1 related effects.

Several mineral dusts that are known to stimulate monocyte/macrophage IL1 relcase, include silica [Schmidt et al., 1984] and asbestos [Hartmann et al., 1984]. Both mRNA expression as well as protein release of IL-1 is upregulated in monocyte's or macrophages stimulated with asbestos [Zhang et al., 1993]. In contrast to TNF and Il-6, the increased mRNA expression of IL- 1 in coal dust stimulated macrophages results not in an increased protein release [Gosset et al., 1991]. This discrepancy has been attributed to an effect of the interleukin-1 receptor antagonist released by the same cells [Vanhée et al., 1994]. Interestingly, Piguet and coworkers showed a significant reduction of silica and bleomycin induced fibrosis with interleukin-1 antagonist [Piguet et al., 1993], although IL-1 
expression was not released in previous experiments [Piguet et al., 1990]. However, Driscoll and coworkers showed that, in line with TNF, IL-1 may be involved in experimental silicosis [Driscoll et al., 1990]. Recently, baseline IL-1 levels were found to be increased in macrophages of subjects chronically exposed to asbestos [Perkins et al., 1993].

Interleukin-6 (IL-6) is produced by most nucleated cells including monocytes, (alveolar) macrophages [Kotloff et al., 1990; May et al., 1989], endothelial cells, fibroblasts and B and T cells [Zitnik and Elias, 1993]. The gene encoding for IL-6 is located on chromosome 7. IL-6 molecules have different sizes ranging from $17 \mathrm{kD}$ to $85 \mathrm{kD}$; monocytes preferentially release $24 \mathrm{kD}$ protein [May et al., 1988]. IL-6 is the common name finally given to a number of factors with various biological effects that proved to be mediated by the identical factor [Zitnik and Elias, 1993]. Previous names include IFN $\beta 2$, 26K, hybridoma-plasmacytoma growth factor (HPGF), B-cell differentiation factor (BCDF), hepatocyte stimulating factor (HSF) and B-cell stimulating factor-2 (BSF-2). IL-6 has a variety of effects that are mediated via specific IL-6 receptors (80kD). Most important function is regulation of B-cells and T-cells. Il-6 is the major regulator of the acute phase response and plays an important role in sepsis [Gauldie et al., 1990]. Several cytokines known to stimulate IL-6 release include IL-1, TNF, PDGF and TGF $\beta$ [Ray et al., 1989]. In IL-6 response to LPS, macrophages are more potent than monocytes, while upon stimulation with IL-1 monocytes release more IL-6 compared to macrophages [Kotloff et al., 1991]. Potent inhibitors of IL-6 include TGF $\beta$, retinoids and glucocorticosteroids [Elias et al., 1991; Zitnik and Elias 1993]. Serum levels of IL-6 has been forwarded as a diagnostic/prognostic marker in cancer [Zitnik and Elias, 1993]. Genetic polymorphism has been demonstrated for IL-6 [Bowcock et al. ,1988].

In the mouse silica-model IL-6 was found in most of the cells from the nodules, however not in serum [Piguet et al., 1990]. Human macrophages release IL-6 in response to coal dust but interestingly not in response to silica or $\mathrm{TiO} 2$ [Gosset et al., 1991]. In addition to TNF and IL-1, macrophages of asbestos exposed subjects produce increased IL-6 [Perkins et al., 1993]. Increased IL-6 expression was found in the lungs of coal miners with CWP and PMF [Vanhée et al. 1995] Recently it was found that IL-6 inhibits collagen degradation [Bienkowski and Gotkin, 1995].

Among all cytokines, Platelet derived growth factor (PDGF) may play a major role in fibrosis, as it is considered as the most potent stimulus of fibroblast proliferation [Ross et al., 1986]. PDGF is a $30 \mathrm{kDa}$ dimeric protein, composed of two $\mathrm{A}$, chains, two $\mathrm{B}$ chains, or an $\mathrm{AB}$ heterodimer. The PDGF-A monomer gene is located on chromosome 7, PDGF-B on chromosome 22. PDGF has originally been isolated from human platelets [Antoniades et al., 1981], from which large amounts are released at sites of tissue injury [Ross et al., 1986]. Various other cells that are known to secrete PDGF include monocytes, macrophages [Shimokado et 
al., 1985] and fibroblasts [Fabisiak et al., 1992]. PDGF mediated effects are mediated by two PDGF receptors, i.e a $\alpha$-hemireceptor, which binds to PDGF-AA or PDGF$\mathrm{AB}$ dimeric proteins and a $\beta$-hemireceptor which only can bind the $\mathrm{BB}$ dimer [Hart et al., 1988]. The $\alpha_{2}$-macroglobulin binds PDGF, and PDGF related effects are dependent on this binding [Bonner and Brody, 1995].

Macrophage release of PDGF may be induced by inorganic particles [Shapira et al., 1992], including coal dust [Vanhée et al, 1995]. PDGF release by fibroblasts is considered to follow an autocrine loop [Battegay et al, 1990]. Although a potent stimulant of fibroblast growth, PDGF has not been shown to induce collagen production by fibroblasts [Clark et al., 1993]. The anti-fibroproliferative action observed with TGF $\beta$ may be closely related to the effects of PDGF. The proliferative effects observed in response to TGF $\beta$ can be blocked by administration of anti-PDGF antibodies [Leof et al., 1986], and TGF $\beta$ is likely involved in the downregulation of the PDGF receptor expression [Battegay et al, 1990]. Recently, Piguet and coworkers showed that PDGF (and TGFß) from blood platelets may play an important role in fibrogenesis [Piguet et al., 1994; Piguet et al., 1995].

\subsubsection{Cytokine networks in mineral dust induced lung disorders}

Although the action mechanisms of cytokines have been extensively studied in vitro, the final responses elicited by cytokines in vivo are dependent on the state of its surrounding tissue, including the presence of other cytokines, soluble cytokine (ant)agonists, as well as the expression of cytokine receptors of target cells [Elias and Zitnik, 1992]. This cytokine network action is considered to play a crucial role in lung disease [Kelley, 1990]. The complexity of the real-life situation in normal or diseased lung may be underscored by the following example.

In addition their stimulating role in fibroblast growth and/or collagen production (see Table 3.3), IL-1 TNF, as well as PDGF increase the synthesis of collagenases [Raghu and Kinsella, 1993]. TNF stimulates monocyte IL-1 release [Danis et al., 1989], induces the biosynthesis of TGF $\beta$ and PDGF [Tracey et al., 1989], but at the same time it is a potent inhibitor of TGF $\beta$ induced collagen production by downregulating the TGF $\beta$ gene expression [Kähäri et al., 1990]. TGF $\beta$ or TNF act synergistically with IL-1 in stimulation of fibroblast IL-6 release and collagen production [Elias et al., 1987; Elias et al., 1991]. However, in combination IL-1 and TNF are less potent in stimulating collagen production than TNF or IL-1 alone [Elias et al., 1987, 1990]. IFN- $\gamma$ reduces the stimulatory effects of TNF or IL-1 [Elias and Kotloff, 1991]. Macrophage TGF $\beta$ expression may be induced by PDGF [Pierce et al., 1989; Pierce, 1990], and TGF $\beta$ in turn stimulates monocytes to produce TNF $\alpha$, IL-1 and PDGF [Roberts and Sporn, 1990]. At the same time TGF $\beta$ suppresses TNF stimulated fibroblast proliferation [Kamijo et al., 1989], and downregulates TNF expression in macrophages [Epsevik et al., 
1987]. PDGF receptors may be either upregulated by IL-1 [Lindrons et al., 1995] or downregulated by TGF $\beta$ [Bonner et al., 1995].

In addition to these and many other possible interactions in the local microenvironment, it should be realized that pathogenic responses in the lung may be related to systemic factors. An important role in lung inflammation is the recruitment of cells of the immune system including "fresh" monocytes/macrophages and neutrophils. Potent factors in this recruitment include the cytokines $\mathrm{L}-8$ [Kelley, 1990], the Macrophage Inflammatory Proteins (MIP) [Wolpe et al., 1987], PDGF [Martinet et al., 1987], TGF $\beta$ [Wahl, 1987], Monocyte Chemotactic Protein-1 [Smith et al., 1995], as well as some other molecules including Leukotriene B4 [Snyderman and Goetzl, 1981]. Recruitment of immune cells, and factors released by these cells, such as reactive oxygen species, IL-4, IL-5, IL-6, IL-8, and interferons [Kelley, 1990] may play an important synergistic or antagonistic role in the fibrogenic effects of mineral dusts. Many of these factors were found to be elevated in rodent models of silicosis, including LTB4 (and PGE2) [Henderson et al., 1991], MIP [Driscoll et al., 1992; 1994] and IL-6 [Piguet et al., 1990]. Rat studies have indicated that recruitment of neutrophils may be closely related to the toxic effects of particles, e.g. of silica versus $\mathrm{TiO} 2$ [Driscoll et al., 1990, 1995]. Although not discussed in detail, these and other cytokines may also be involved in nonpneumoconiotic effects in coal workers.

\subsection{Collagen.}

\subsubsection{Collagens in the lung}

The connective tissue of the lungs consists of collagen, elastic fibers and proteoglycans. At least 15 types of collagen have been described to date [Bienkowski, 1991; Prockop and Kivirikko, 1995], the lung interstitium containing collagen types I, III, V, VI and XII [Bienkowski, 1991]. Interstitial collagens are important determinants of structural and mechanical properties of the lung, which normally contains about 100 to $200 \mathrm{mg}$ collagen (dry weight). Interstitial collagens of the lung are synthesized by fibroblasts [Hance et al, 1976], endothelial cells [Makarak et al., 1984], pleural mesothelial cells [Rennard et al., 1984] and alveolar epithelial cells [Sage et al., 1983]. A collagen is a triple helix of three polypeptide subunits known as $\alpha$-chains, and contains high amounts of (hydroxy)proline. A major part of the $\alpha$-chain subunits consists of repetitive amino-acid triplets containing a glycine followed by two random amino-acids. Collagen trimers are either homotrimers, i.e. composed of three identical subunits or heterotrimers, i.e. composed of two or three different subunits.

The most abundant collagens are type I and III which copolymerize into collagen fibrils and are normally found in a ratio of $3: 1$ to $6: 1$ in the interstitium [Madri and Furthmayer, 1980]. Collagen I represents about 60 to $70 \%$ of the lung collagen content. Collagen I and III are mainly produced by fibroblasts, but 
additionally can be produced by endothelial cells, epithelial cells, and smooth muscle cells. Collagen type V is often associated on the surface of the large I/III fibers, whereas type VI forms an independent beaded network in the interstitium [Bienkowski, 1991]. Collagen V and VI, which are both produced by fibroblasts generally contain less than $5 \%$ of the lung. Collagen type IV, which represents about $5 \%$ of the lung mass is not found in the interstitium, but is an important component of the basement membrane of the lung. Other components of the basement membrane, which forms an important particle barrier to the interstitium, are laminin and proteoglycans. Several components of the extracellular matrix have chemotaxic activity towards inflammatory cells and fibroblasts, such as collagen I, III and VI, laminin and fibronectin [Postlethwaite et al., 1976; Laskin et al., 1986].

\subsubsection{Collagen synthesis and degradation}

The normal lung is characterized by on-going processes of synthesis and degradation of collagens. Increased collagen deposition -the hallmark of fibrosisis believed to result from an impaired balance between these processes [Snyder, 1988; Murray and Laurent 1988]. Collagens are synthesized and secreted as higher molecular weight molecules known as procollagens [Bienkowski, 1991]. Procollagens are trimers of pro $\alpha$-chains, with two propeptides at each end, i.e. a $\mathrm{C}$-terminal propeptide and a $\mathrm{N}$-terminal propeptide. The $\mathrm{C}$-terminal propeptides are registration sites for individual pro- $\alpha$-chains and initiate the folding of the triple helix. During posttranslational modification of the collagen, hydroxyproline is formed by enzymatic hydroxylation of proline during its incorporation into a peptide chain. This reaction is mediated by prolylhydroxylase. Since no other known protein contains significant amounts of hydroxyproline, it is generally used as a marker for collagen, and increased lung hydroxyproline content is considered as a marker of fibrosis [Murray and Laurent, 1988]. Both propeptides, which allow the collagen to be soluble within the cell, are cleaved by specific peptidases after the procollagen molecule is secreted. After its cleavage, the N-terminal procollagen is believed to play a role in a feedback inhibition of procollagen translation process [Bienkowski, 1991]. Collagen molecules in a fibril are arranged in parallel. Lysine and hydroxylisine residues are responsible for the intra- and intermolecular cross-links between the individual $\alpha$-chains of the collagen units. Collagens bind to cell surface receptors as well as to fibronectin in the matrix. In normal lung diameter of collagens are uniform, but in fibrotic lung fibrils are often frayed and have irregular contours [Fukuda et al., 1985]. It is not known whether this is a result of degradation of normal fibrils or as a result of deranged fibrilogenesis.

The degradation of interstitial collagens is guided by collagenases that are synthesized by interstitial cells as well as by macrophages [Welgus et al., 1985; Garbisa et al., 1986; Hibbs et al., 1987] and neutrophils [Harris et al., 1984]. 
Collagenases produced by fibroblasts and alveolar macrophages are about equally active against collagens I and III, while collagenases synthesized by neutrophils degrade collagen I much more rapidly than collagen III [Horwitz et al., 1977]. Collagenase can be activated by oxidative processes [Weiss et al., 1989] and has been detected in bronchoalveolar lavage fluid of patients in idiopatic pulmonary fibrosis and in sarcoidosis [Gadek et al., 1979; O'Connor et al., 1988]. However others have found that fibroblasts obtained from fibrotic subjects contain less collagenase activity compared to controls [Selman et al., 1991]. Collagenase synthesis is increased by IL-1, TNF and PDGF [Raghu and Kinsella, 1993]. Other collagen degrading proteases of the lung include neutrophil elastases, cathepsin $G$ and gelatinase [Gadek et al., 1980; Janoff, 1985; Vogelmeier et al., 1991] and macrophage elastases [Senior et al., 1989]. Proteases are believed to play an important role in emphysema [Janoff, 1985] and bronchitis [Stockley et al., 1983].

\subsubsection{Type III procollagen peptide}

Collagen type III is a homotrimer composed of three $\alpha 1$ chains. The $\mathrm{N}$-terminal propeptide of the procollagen can be detected in bronchoalveolar lavage fluid and in serum. In areas of early fibrosis more type III collagen than type I collagen is found while in later stages of fibrosis, type I predominates [Bateman et al., 1981; Raghu et al., 1985]. In a bleomycin model of fibrosis, type III procollagen mRNA is increased in individual cells of affected areas, measured in situ [Shahzeidi et al., 1993]; In later study also increased collagen I was observed, preceding the increases in type III procollagen mRNA [Shahzeidi et al., 1993]. Altered ratios of collagen I and collagen III that have been observed during development of fibrosis may be explained by three different mechanisms, i.e. (1) a switch of fibroblasts that initially synthesize collagen III and later switch to collagen I production, (2) proliferation of subsets of fibroblasts with distinct production of type I and III collagens, or (3) increased degradation rate of collagen III compared to type I collagen [Raghu and Kinsella, 1993]. N-terminal type III procollagen peptides (PIIIP) have been measured in bronchoalveolar lavage or serum of subjects with fibrotic lung disease including idiopatic pulmonary fibrosis [Low et al., 1983; Cantin et al., 1988] and asbestosis [Okazaki et al., 1983; Cavalleri et al., 1991]. Previously, serum PIIIP was found to be related to radiological stage of CWP [Janssen et al., 1992a]. Correlations between serum and lavage PIIIP levels have been studied by Low and coworkers [Low et al., 1989; Low et al., 1992] and serum PIIIP has been forwarded as a marker of early clinical diagnosis in fibrotic lung disease [Low et al., 1992; Janssen et al., 1992] and/or mineral dust exposure [Cavalleri et al., 1993; Becklake et al., 1993]. 



\section{Chapter 4}

\section{DESIGN OF THE STUDIES}

\subsection{Description of the study population and of the biomarkers evaluated}

In line with biological mechanisms involved in mineral dust induced lung disorders (chapter 3), the specific studies in this thesis are subdivided into three parts. In separate chapters (1) oxidant/antioxidant parameters, (2) cytokines, and (3) type III collagen will be discussed in view of their applicability as biological markers. As already outlined for some of the parameters their candidacy as biomarkers of CWP was forwarded from previous cross-sectional surveys by our laboratory. Three study groups of miners from the Belgian coal mining region "Kempen" were recruited for a follow-up. In the first study, spontaneous and stimulated release of TNF by peripheral blood monocytes was measured in 66 coal workers and 12 controls. Monocytes were stimulated with coal dust, silica and endotoxin (LPS), and the release was related to severity of CWP based on chest radiograph classification [Borm et al., 1988]. In the second study, antioxidants in red blood cells (GSH, GSSG, GST, Gpx, SOD) and serum (Vitamins A and E) of 73 coal workers and 16 controls were analysed and related to chest radiographs [Engelen et al., 1990; Evelo et al., 1993]. The third study described measurement of serum PIIIP in a subgroup of the latter study group [Janssen et al., 1992]. The evaluation of these parameters in a the follow-up design are discussed in chapter 7 (antioxidants), chapter 8 (TNF), and in the chapters 11 and 12 (PIIIP). New markers in the follow-up concerning cytokinerelated mechanisms include plasma soluble TNF receptors (sTNF-R55 and sTNFR75) (chapter 9) and monocyte release of TGF $\beta$ and IL-6 (chapter 10). With regard to oxidative stress responses, serum total radical antioxidant parameter (TRAP) (chapter 5) as well as oxidative DNA damage (8-oxodG) in lymphocytes (chapter 6) are evaluated. Figure 4.1 shows how the markers evaluated in this thesis should be seen in the pathogenesis of mineral dust induced lung disorders as outlined in chapters 2 and 3.

The particular choice of new parameters was made in view of their relation with the markers previously determined (TNF, AOE), in order to elucidate their possible involvement in coal dust induced lung disorders (chapter 3 ) as well as their candidacy as biomarkers in mineral dust exposure and disease. Both soluble TNF receptors (R75 and R75) are determined in the plasma of coal workers because of their natural occurrence in human plasma and the proposed regulatory role in the systemic effects of TNF [Jäätelä, 1990; Ulich et al., 1993], and their antifibrogenic effects observed in mineral dust induced fibrosis [Piguet et al., 1994]. Monocyte release of TGF and IL-6 are included, since in line with TNF (1) they are both released from coal dust stimulated alveolar macrophages [Gosset 


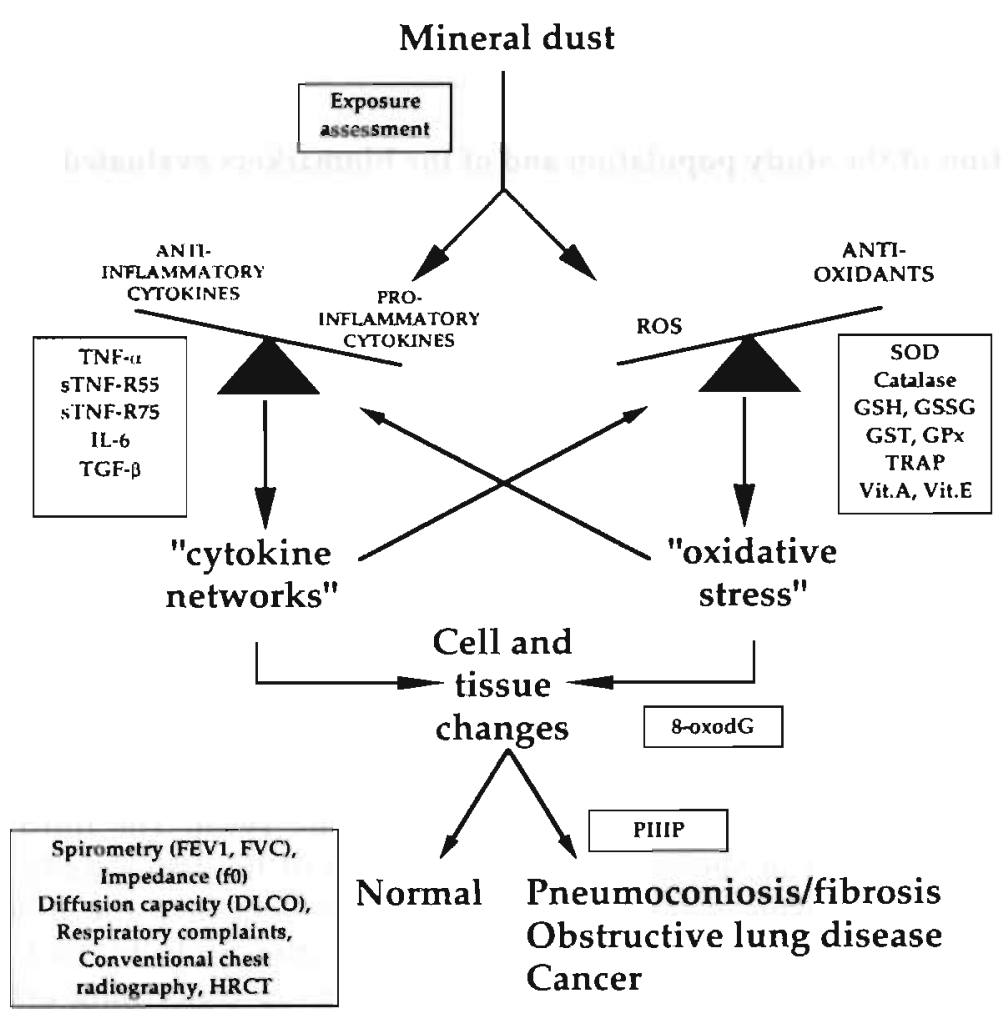

Figure 4.1 The integration of biological mechanisms that have been used in this thesis for the generation and testing of biomarkers for mineral dust induced health effects. Markers are indicated in rectangulars. The two triangles indicate the main balances that can be tipped during ongoing inhalation and deposition of dust particles. Disturbance of the balance between reactive oxygen species (ROS) and antioxidants may result in oxidative stress responses [Janssen et al., 1993]. Proinflammatory cytokines may trigger cells and tissue to become activated, proliferated and/or differentiated, while anti-inflammatory cytokines may act as feedback mechanisms to these effects [Kelley, 1990]. Both pathways are interrelated, since ROS generating cells (e.g. neutrophils) may be recruited by cytokines, cytokines are known to modulate antioxidant expression and activities within cells, and ROS may either amplify or counteract cytokine responses. Both oxidative and cytokine mediated pathways play a role in a number of lung disorders including alveolitis, fibrogenesis, lung function decline, chronis bronchitis, and cancer. During the development or progression of these disorders, both balances may be affected, and thus likely modify pathological responses. In this cascade of events, presented markers may reflect (chronic) exposure to mineral dust, (non)pneumoconiotic pathogenesis resulting from this exposure, and/or both in the absence or presence of disease- individual differences in susceptiblity to mineral dust induced lung disorders. 
et al., 1991; Vanhée et al., 1994], and (2) their presence is demonstrated in lungs of fibrotic patients [Broekelman et al., 1991; Vanhée et al., 1995]. Formation of 8oxodG in lymphocyte DNA is determined in order to study whether the changes in antioxidant status observed in animal models of mineral dust exposure [Janssen et al., 1994] as well as in humans [Borm et et al., 1988] may cause significant oxidative effects in subjects chronically exposed to mineral dusts. Furthermore, measurement of oxidative DNA damage in the present study group (i.e. in exposed subjects plus pneumoconiotic subjects) could provide insight in the role of the proposed carcinogenicity in existing pneumoconiotic disease [McDonald, 1989]. TRAP was determined to evaluate the role of plasma as antioxidant pool to cope with pathogenic effects caused by reactive oxygen species in the lung, and to study their relation with antioxidants previously measured in coal workers [Borm et al., 1988].

Although specific hypotheses have been generated for specific candidate markers, the follow-up of these cohorts was carried out with a number of general purposes:

(1) Determination of development and progression of pneumoconiosis during the follow-up period by longitudinal evaluation of chest radiographs.

(2) Renewed measurement of TNF, PIIIP and antioxidants (AOE) to determine five-year changes in these markers in relation to changes in disease stage and reproducibility of the cross-sectional observations.

(3) Inclusion of new candidate markers (TGF, IL-6, TRAP, 8-oxodG) and crosssectional and/or retrospective data analysis of these parameters.

(4) Inclusion of accurate exposure assessment (i.e. cumulative dust exposure) to investigate the role of exposure in relation to all parameters, including candidate markers and disease (progression).

(5) Inclusion of lung function data and respiratory complaints to assess for nonpneumoconiotic disease and/or confounders.

(6) Inclusion of HRCT in a subgroup, to detect and quantify early stage fibrosis.

(7) Comparison of markers to a carefully selected control group with known occupational and medical history and lifetime smoking habits.

\subsection{Peripheral blood versus the lung}

Bronchoalveolar lavage or lung biopsies would be ideal methods to screen for biomarkers in relation to the "target tissue" studied. However, validated biomarkers should be easily applicable in large scale routine surveys, i.e. the biomarkers should be minimally invasive or noninvasive [Weill, 1993]. 
Although, most interactions and the eventual effects comprise the lung tissue, all markers evaluated in this thesis are measured in the peripheral blood obtained by venapuncture. The use of peripheral blood is underscored by the following observations:

(1) Blood sampling is a relative non-invasive method to retrieve cells and fluid, in comparison to bronchoalveolar lavage.

(2) Red blood cells are considered as important antioxidant carriers for the lung [van Asbeck et al., 1984; Engelen et al., 1990], and since mature erythrocytes contain no nucleus or organelles they are not capable of restoring their antioxidant levels by de novo synthesis of lost proteins.

(3) Reduced antioxidant levels (vitamin A, C, E) in human plasma are considered as risk factors in lung disease [Morabia et al., 1989; Schwartz and Weiss, 1994; Britton et al., 1995].

(4) Peripheral blood lymphocytes may be considered as a surrogate tissue for target cells from which DNA may be obtained relatively non-invasive.

(5) Peripheral blood monocytes are recruited to the lung at inflammatory sites and differentiate into mature alveolar macrophages. Increased recruitment of monncytes from the blood (by chemotactic factors) is known to occur in pneumoconiotic disease (see chapter 3).

(6) In contrast to alveolar macrophages, peripheral blood monocytes are not directly exposed to particles and toxicants in the lung. Ex-vivo stimulation of monocytes is thus less likely to be biased by in vivo stimulatory artefacts.

(7) Cytokine release as well as mechanisms leading to increased cytokine release in the lung may be involved in extrapulmonary effects by means of priming mechanisms [Mohr et al., 1991; Mohr et al., 1992]. Soluble cytokine receptors released from blood cells may comprise a major systemic source to either reduce or amplify these effects.

(8) Procollagen peptides are abundant in human plasma and are specific due to collagen metabolism, and correlations between PIIIP in lavage fluid and serum have been reported in fibrotic lung disease [Low et al., 1983]. 
PART 1

Oxidative damage and antioxidant defense 
193189

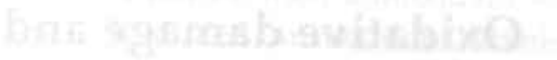

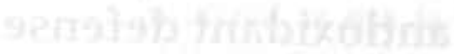




\title{
Chapter 5
}

\section{SERUM TOTAL RADICAL-TRAPPING ANTIOXIDANT PARAMETER (TRAP) IN COAL WORKERS.}

\author{
Roel PF Schins ${ }^{1}$, Thim JJM Derhaag1, Jan De Jong ${ }^{2}$, Aalt Bast ${ }^{2}$, Paul JA Borm¹. \\ 1 Department of Health Risk Analysis and Toxicology, University of Limburg, \\ PO BOX 616, $6200 \mathrm{MD}$, Maastricht, the Netherlands. \\ 2 Department of Pharmacochemistry, Free University, De Boelelaan 1083, \\ $1081 \mathrm{HV}$ Amsterdam, the Netherlands.
}

Published in: Clinical Chemistry 1994;40:1470-2.

\begin{abstract}
Formation of reactive oxygen species in response to (mineral) dust exposure is a welldescribed phenomenon in processes ultimately leading to lung fibrosis. We determined serum total radical-trapping antioxidant parameter (TRAP) and total red blood cell glutathione in coal miners with or without coal workers' pneumoconiosis (CWP) and in non-dust-exposed control subjects. Total serum antioxidant capacity was significantly increased in new cases of coal workers' pneumoconiosis and was inversely related to individual cumulative exposure in cases without "active" fibrosis. These findings are in line with the hypothesis that oxidative stress is present in the lung during active phases of coal workers' pneumoconiosis.
\end{abstract}

\section{Introduction}

Reactive oxygen species (ROS) play an essential mediating role in the damage to many cell types in the lung in response to inhalation of mineral dusts (asbestos, silica, coal). Both physical and chemical surface properties of the inhaled dust particles and oxidative burst by polymorphonuclear granulocytes, alveolar macrophages and other inflammatory cells are associated with the formation of free radicals such as $\mathrm{OH} \bullet, \mathrm{H}_{2} \mathrm{O}_{2}$ and $\mathrm{O}_{2}^{-}$- [Borm et al., 1990; Janssen et al., 1992b]. In concert with other biological mechanisms such as fibroblast activation, chemotaxis and secretion of cytokines, ROS and lysosomal enzymes may ultimately lead to pulmonary fibrosis. Fortunately, the lung possesses an elaborate defence systems against ROS, commonly summarized under the name antioxidant system (AOS) [Janssen et al., 1992b].

In the course of our studies seeking to explain why certain coal workers develop coal workers' pneumoconiosis (CWP) whereas others -equally exposed- 
do not [Borm et al., 1990] we observed that many components of the AOS were related to the stage of pneumoconiosis [Engelen et al., 1990]. Previously, most of the AOS markers were measured in red blood cells, whith only vitamins A and E being measured in serum. The aims of the present study were to determine the total radical-trapping antioxidant parameter (TRAP) in serum of coal workers and to test consistency with our previous findings.

\section{Subjects, materials and methods}

Participants in this study were 104 coal miners from our previous cohorts. Informed consent was obtained, and the study was approved by the ethical standards of our institution's responsible committee according to the Helsinki Declaration of 1975, as revised in 1983. Pneumoconiotic stages of both the original cross-sectional studies [Borm et al., 1990; Engelen et al., 1990] and the follow-up, were determined by chest $x$-ray classification according to the International Labour Organization (ILO) criteria by a panel of three physicians. Five-year development (among 80 reference miners) or progression (in 24 miners with (WP) was deduced from paired readings of each individual's 1987 and 1992 chest x-rays. In addition 26 age matched male controls without any occupational dust exposure were randomly selected from the general population in the same area. Subjects with a known history of pulmonary diseases or current allergies or chronic diseases of liver, kidney, or bladder were excluded. Smoking habits and medical history were obtained from questionnaires. Occupational exposure was defined as years underground and as cumulative dust exposure. Cumulative exposure, expressed as hours exposed $x$ grams of dust per cubic meter sampled air, was estimated from each individual's job history and from dust measurements at various sites of the coal mine.

Total glutathione in red blood cells was measured according to Anderson [Anderson, 1985]. Serum TRAP was measured as originally described by Wayner et al. [Wayner et al., 1987]. Briefly, to a closed compartment at $37^{\circ} \mathrm{C}$ containing an oxygen electrode (YSI Model 5331 Probe; YSI Model 3500 Biological Oxygen Monitor; Yellow Springs Instruments, Yellow Springs, OH), we added $15 \mu \mathrm{L}$ of serum mixed with linoleic acid (25/1, by vol; Aldrich Chemical Co., $99 \%$, cat. no. 22.392-7) and 15 of 2,2'-Azobis(2-amidinopropane) $\mathrm{HCl}(0.25 \mathrm{~mol} / \mathrm{L}$; Polysciences, cat. no. 08963 ) to $1.5 \mathrm{~mL}$ Phosphate Buffered Saline (140 mmol/L NaCl, $\mathrm{pH}=7.4$ ). After the interval of inhibited peroxidation (i.e., serum lagtime), we added $25 \mu \mathrm{L}$ of a water-soluble vitamin E-analog, Trolox (6-hydroxy-2,5,7,8 tetramethylchroman-2-carboxylic acid), (133mmol/L; Aldrich Chem. Co., 97\%, Cat. no. 23.881-3) as a standard (i.e., Trolox-lagtime). TRAP is defined as the amount of peroxyl radicals captured non-enzymatically per liter of solution: 
TRAP $=(\mathrm{T}$-serum $/ \mathrm{T}$-trolox $) \cdot \mathrm{n} \cdot \mathrm{f} \cdot[$ Trolox $]$

where, T-serum is the lag time of the serum sample; T-trolox is the lag time of Trolox; $\mathrm{n}$ is a stoichiometric factor, indicating the number of radicals (mol) captured by an antioxidant (i.e., 2.0 for Trolox); and $f$ is the dilution factor of serum vs Trolox.

Table 5.1 Characteristics of the control and study groups, and corresponding measures of red blood cell glutathione and serum TRAP.

\begin{tabular}{lccccc} 
& \multicolumn{2}{c}{ Controls } & \multicolumn{2}{c}{ Reference miners } & \multicolumn{2}{c}{ Miners with CWP } \\
\hline & & $\mathrm{NP}$ & $\mathrm{P}$ & $\mathrm{NP}$ & $\mathrm{P}$ \\
$\mathrm{n}$ & 26 & 76 & 4 & 11 & 13 \\
Age, years & $50.5(1.01)^{a}$ & $47.7(0.57)$ & $44.5(2.84)$ & $50.0(1.98)$ & $52.1(1.13)^{a}$ \\
Exposure & & & & & \\
Underground, years & - & $22.3(0.44)$ & $23.8(2.84)$ & $23.7(0.95)$ & $25.8(1.41)$ \\
Cumulative dust, gh/m 3 & - & $89.6(6.4)$ & $130.0(23.7)$ & $127.4(13.3)$ & $132.6(16.7)$ \\
Glutathione, mmol/gHb & $6.03(0.18)$ & $5.72(0.11)$ & $6.15(0.46)$ & $5.81(0.34)$ & $6.09(0.28)$ \\
TRAP, $\mu \mathrm{mol} / \mathrm{L}$ & $924(25)^{b}$ & $926(31)^{b}$ & $1108(66)^{c}$ & $944(52)$ & $876(28)$ \\
\hline
\end{tabular}

Values are mean (SEM): $\mathrm{P}=$ progression (or development) of pneumoconiosis; $\mathrm{NP}=$ no progression.

a Significantly different from reference miners ( $\mathrm{P}<0.05$, Kruskall-Wallis).

${ }^{b}$ TRAP measured in only 5 controls and $13 \mathrm{NP}$-reference miners.

${ }^{c}$ Significantly different from all other groups ( $P<0.05$, Kruskall-Wallis).

\section{Results}

No significant differences in TRAP concentrations (Table 5.1) were observed between non-dust-exposed controls $(n=5)$, reference miners $(n=13)$ and miners with CWP $(n=28)$ ( $P>0.7$, ANOVA). The CWP group showed an inverse relation between serum TRAP and pneumoconiotic stage (Spearman's $\rho=-0.39$; $\mathrm{P}<0.05)$; however, the variation in TRAP was large, so that differences between the stages were not significant $(P=0.22)$. Although the same trend is seen in TRAP and glutathione data, there was no significant correlation between serum TRAP and red blood cell glutathione concentrations $(n=46, r=0.016, P>0.9)$.

During this 5-year follow-up, either several subjects developed CWP newly or there was progression of existing CWP. Subjects with newly developed pneumoconiosis $(n=4)$ had significantly higher TRAP concentrations than the control miners who did not develop CWP (Table 5.1). Glutathione levels were not significantly increased in those subjects but showed a similar trend. 
Interestingly, TRAP concentrations and cumulative dust exposure were correlated in the miners group with CWP, but this was significant only in the non-progressed individuals $(n=11, r=-0.6, P<0.05)$.

\section{Discussion}

Previously we demonstrated a relationship between several factors of the antioxidant defense system and pneumoconiotic stage [Engelen et al., 1990; Evelo et al., 1993]. We observed that red blood cell glutathione concentration [Engelen et al., 1990] as well as red blood cell glutathione-S-transferase activity [Evelo et al., 1993] were decreased in coal miners at early stages of CWP (ILO classification 0/1$1 / 2$ ). On the other hand, glutathione peroxidase activity in red blood cells was increased in the early stages of CWP [Engelen et al., 1990]. From these findings, we hypothesized that the variations in red blood cell antioxidant factors reflected the oxidative stress imposed by the inflammatory processes in the lung [Borm et al., 1990]. The present data failed to confirm the decrease in (total) glutathione in red blood cells in the early stage of CWP; the fact that miners in this study were all retired and therefore had not been exposed for at least the preceding 2 years might explain this discrepancy. In new cases of CWP and those miners whose disease progressed over 5 years higher concentrations of total glutathione were observed. Interestingly, total serum antioxidant capacity undergoes changes similar to red blood cell glutathione, although there is no correlation between these two markers on an individual basis. Moreover, TRAP appeared to be inversely related to individual (cumulative) exposure but only in miners without "active" fibrosis. Because TRAP is the end result of several antioxidants, we have no information on the causal factor(s) involved but consider vitamin A and $E$ unlikely candidates because of our previous results [Engelen et al., 1990]. The present findings are consistent with our previous hypothesis that during the first phases or progression of CWP, oxidative stress is present in the lung. Either a decrease or increase in anti-oxidant factor(s) can be observed, the change being dependent on the relative impacts of (a) the extra load on the antioxidant system and (b) the extra synthesis of antioxidant factors.

Acknowledgements. We thank all coal miners and the Kempense Steenkoolmijnen NV for their willingness to cooperate in this study, Luc Lenaerts (MD) for blood sampling and reading of the $x-$ rays, Marc van Sprundel (PhD) and Luc Mariën (MD) for reading the $x$-rays. This study is supported by grant no. 7263/03/092 of the European Community for Steel and Coal. 


\title{
Chapter 6
}

\section{OXIDATIVE DNA DAMAGE IN PERIPHERAL BLOOD LYMPHOCYTES OF COAL WORKERS.}

\author{
Roel PF Schins, Pauline AEL Schilderman, Paul JA Borm. \\ Department of Health Risk Analysis and Toxicology, University of Limburg, \\ PO BOX 616, $6200 \mathrm{MD}$, Maastricht, the Netherlands.
}

Published in: International Archives of Occupational and Environmental Health 1995;67:153-7.

\begin{abstract}
Reactive oxygen species are important mediators of both mineral dust induced (malignant) lung disease and in vitro DNA-damage. Therefore, we studied in vivo oxidative DNA-damage in coal workers who had been chronically exposed to silicacontaining dust. In peripheral blood lymphocytes of 38 retired coal miners (eight with coal workers' pneumoconiosis, 30 references) and 24 , age matched, non-dust-exposed controls 7-hydro-8-oxo-2'-deoxyguanosine (8-oxodG) was determined by reversed phase high-performance liquid chromatography (HPLC) with electrochemical detection detection (ECD). The ratio of 8-oxodG residues to deoxyguanosine $(\mathrm{dG})$ was related to individual cumulative dust exposure estimates and pneumoconiotic stage as established by chest radiography. The ratio of 8-oxodG to $\mathrm{dG}\left(\times 10^{-5}\right)$ in lymphocytes did not differ between miners with coal workers pneumoconiosis $(2.61 \pm 0.44)$ or miners without coal workers' pneumoconiosis $(2.96 \pm 1.86)$. However, oxidative DNA-damage in all miners was higher than in the non-dust-exposed controls (1.67 \pm 1.31$)$. 8-oxodG/dG was not related to individual cumulative coal dust exposure, age or smoking (pack-years) when evaluated by multiple linear regression. We suggest that oxidative damage to the DNA of peripheral blood lymphocytes may be introduced by increased oxidative stress responses in subjects chronically exposed to mineral dusts. Whether this is an important pathway in the suggested carcinogenicity of silica is still an open question.
\end{abstract}

\section{Introduction}

It is well known that occupational exposure to mineral dusts can lead to the development of fibrotic lung diseases (i.e. asbestosis, silicosis, coal workers pneumoconiosis). Many studies have demonstrated that reactive oxygen species (ROS) are involved in the biological effects of these mineral dusts [Mossman and Marsh 1991; Janssen et al. 1993]. Both physical and chemical surface properties of the inhaled dust particles and the oxidative burst by polymorphonuclear 
granulocytes, alveolar macrophages and other inflammatory cells are associated with the formation of free radicals such as $\mathrm{OH} \bullet, \mathrm{H}_{2} \mathrm{O}_{2}$ and $\mathrm{O}_{2}{ }^{-}$. [Janssen et al. 1993]. The involvement of elaborate antioxidant defence systems against the harmful effect of ROS, has been studied by our laboratory to explain for differences in susceptibility to the development and progression of Coal Workers Pneumoconiosis (CWP) in coal workers [Borm et al. 1986; Engelen et al. 1988; Evelo et al. 1993; Schins et al. 1994a] and in experimental fibrosis in rats after in vivo exposure to asbestos and cristobalite [Janssen et al. 1992b, 1994a].

On the other hand, chronic inhalation of mineral dusts, especially asbestos and to a lesser degree silica, has also been associated with the development of lung cancer [McDonald 1989; Mossman et al. 1990; Holland 1990; Craighead 1992]. Since ROS are important mediators of in vitro DNA-damage, and related to carcinogenesis [Trush and Kensler 1991; Janssen et al. 1994b], it is suggested that oxidant generation may also play a crucial role in carcinogenic action of mineral dusts [Ghio et al. 1990; Janssen et al. 1993]. A particular type of DNA-damage which is directly related to the action of ROS, is the C8 oxidation of deoxyguanosine (dG). The resulting 7-hydro-8-oxo-2'-deoxyguanosine (8-oxodG) may be used as a biological marker of oxidative stress to DNA [Kasai et al. 1986; Kuchino et al. 1987)]. Recently, Hanaoka et al. [1993] have measured 8-oxodG/dG ratios in lymphocytes of asbestosis patients, to study the in vivo significance of the oxidative stress believed to be involved during asbestos exposure and in asbestosis. In the present study we describe the determination of 8 -oxodG/dG ratios in peripheral blood lymphocytes of coal miners with or without CWP and in controls not occupationally exposed to dust. The purpose of the study was to evaluate 8 -oxodG/dG analysis in peripheral blood lymphocytes as a biological marker of the cumulative (silica-)dust exposure or on the other hand of the fibrotic effects (pneumoconiosis) which result from chronic coal dust exposure.

\section{Materials and methods}

As a part of a prospective cohort study among coal workers [Borm 1994], the ratio of 8-oxodG/dG was determined in peripheral blood lymphocyte DNA of coal miners $(n=38)$ and non-dust-exposed controls $(n=24)$. Prior to the blood sampling, a questionnaire, including informed consent was obtained from each participant. On the day of blood sampling, a chest radiograph was made of each miner, and a personal interview to confirm answers in the questionnaires and to check the medical files and job history. All subjects were classified as smokers, nonsmokers, former smokers or never smokers. Amount smoked was expressed in pack-years as described previously [Jorna et al. 1994a], after verification of smoking reporting during the personal interview and comparison with data from the previous cross-sectional studies [Borm et al. 1990] to minimize 
reporting bias. The same was done for medical history. Pneumoconiotic staging of the coal miners was achieved by classification of chest radiographs by three occupational physicians according to a standard protocol of the International Labour Organization [I.L.O. 1980] and has been described elsewhere [Schins et al. 1994b]. The cumulative dust exposure was determined from job-exposure matrices as described previously [Jorna et al. 1994a].

Isolation of DNA from peripheral blood lymphocytes

Peripheral blood lymphocytes were isolated as follows: blood $(3 \times 10 \mathrm{ml}$, EDTA tubes) was sampled, stored at $4^{\circ} \mathrm{C}$, and subsequently left overnight $(20.00 \mathrm{~h}$ p.m.$8.00 \mathrm{~h}$ a.m.) at room temperature. Lymphocytes were then isolated by buoyant density centrifugation (Lymphoprep-Nycomed), adjusted to concentrations of $5 \times 10^{6}$ cells $/ \mathrm{ml}$ and seeded in portions of $0.5 \mathrm{ml}$ to tissue culture dishes (Costar TC-24) to allow adherence during 30 minutes incubation in RPMI 1640 supplemented with $10 \%$ FCS. All nonadjacent cells (repetitive agitation and washing) from each subject were pooled, washed twice in PBS (10 min., $800 \mathrm{~g}$ ) and stored at $-70^{\circ} \mathrm{C}$ until isolation of cellular DNA according to the method of Gupta [Gupta 1984], as described previously [Schilderman et al. 1993]. Briefly, cells were homogenized in $1 \%$ SDS/1 mM EDTA and subsequently incubated with proteinase $\mathrm{K}$. The homogenate was succesively extracted with $1 \mathrm{vol}$ of phenol, 1 $\mathrm{vol}$ of phenol/chloroform/isoamyl alcohol $(25 / 24 / 1 ; \mathrm{v} / \mathrm{v} / \mathrm{v})$ and $1 \mathrm{vol}$ of chloroform/isoamyl alcohol (24/1). After addition of $0.1 \mathrm{vol}$ of $3 \mathrm{M} \mathrm{NaAc}$ $(\mathrm{pH}=6.0)$, nucleic acid was precipitated with $2 \mathrm{vol}$ of ice-cold ethanol, washed with $70 \%$ ethanol to remove salt and dissolved in $5 \mathrm{mM}$ Tris- $\mathrm{HCl}(\mathrm{pH}=7.4)$ and $1 \mathrm{mM}$ EDAT ( $\mathrm{pH}=7.4)$. RNA was destroyed by addition of RNase T1 (150U/mg DNA) and RNase A ( $300 \mu \mathrm{g} / \mathrm{mg}$ DNA) during $30 \mathrm{~min}$ of incubation at $38^{\circ} \mathrm{C}$. After extraction of the digest with 1 vol chloroform/isoamyl alcohol (24/1), DNA was recovered as described above and dissolved in $5 \mathrm{mM}$ Tric- $\mathrm{HCl}(\mathrm{pH}=7.4)$. DNA concentration was assayed spectrophotometrically. The DNA was digested to deoxynucleosides by treatment with nuclease P1 (25 U/mg DNA) and alkaline phosphatase [Lutgerink et al. 1992]. DNA was isolated from lymphocytes of controls and miners in randomised order. Samples with a DNA yield below 40 $\mu \mathrm{g}$ were excluded from analysis. DNA yield did not differ between miners and controls and not related to day of isolation.

\section{HPLC/ECD analysis of 8-oxodG}

The ratio of 8-oxodG/dG was determined according to Floyd et al. [1986] as described previously [Schilderman et al. 1993]. Briefly, 8-oxodG was detected by HPLC/ECD analysis, using a Spectroflow 480 Solvent delivery system coupled with a Kratos spectroflow 783 programmable absorbance detector and an Antec electrochemical detector CU-04-AZ $(850 \mathrm{mV})$. A supelcosil LC- $18 \mathrm{~S}$ column (supelco: $250 \times 4.6 \mathrm{~mm}$ ) was used in conjunction with a $5 \mu \mathrm{m}$ diameter guard 
column. The mobile phase consisted of $10 \%$ aqueous methanol containing sodium acetate $(25 \mathrm{mM})$, citric acid $(12.5 \mathrm{mM}), \mathrm{NaOH}(30 \mathrm{mM})$ and acetic acid (10 $\mathrm{mM}$ ). Elution was performed at a flow rate of $1.0 \mathrm{ml} / \mathrm{min}$. The lower limit of detection was $40 \mathrm{fmol}$ absolute for 8 -oxodG, or 1.5 residue $/ 10^{6} \mathrm{dG}$ requiring a minimum yield of $35 \mu \mathrm{g}$ DNA per sample. dG was simultaneously monitored at $260 \mathrm{~nm}$. Oxidative DNA damage was expressed as the ratio of 8-oxodG to dG. Samples were analyzed randomly with a calibration curve and a selection of controls on each run. 8-oxodG/dG ratios were not dependent on day of isolation, day of HPLC analysis, or DNA yield.

\section{Statistical evaluations}

Study group characteristics were evaluated by the Student's t-test and the non parametrical Mann-Whitney U-test, i.e. age, pack-years smoked, exposure and oxidative DNA damage. The $\chi^{2}$-test was used to evaluate smoking and medication. Relations between 8-oxodG/dG and age, pack-years, and exposure parameters (i.e. miners only) were evaluated by (multiple) linear regression models (stepwise regression analysis). Statistical evaluation was made using STATGRAPHICS version 6 (Manugistics Inc., Rockville Maryland 20852. USA).

\section{Results}

Fig. 6.1 illustrates that 8 -oxodG/dG ratios in the miners were significantly higher than in the non-exposed controls $(\mathrm{p}<0.01)$. All other group characteristics and ratio's of 8-oxodG/dG are shown in Table 6.1.

Figure 6.1 Ratio of $80 \times 0 \mathrm{dG} / \mathrm{dG}$ in peripheral blood lymphocytes of 24 non-dust-exposed controls and of 38 coal miners

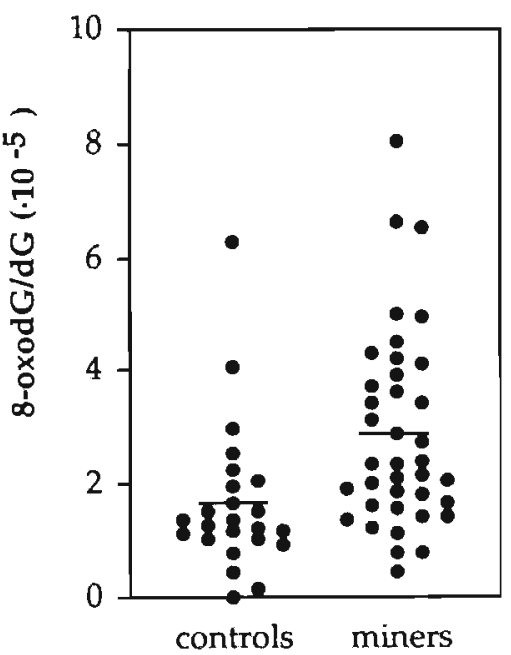


Although miners with CWP were significantly more exposed on average (see Table 6.1), 8-oxodG/dG ratio's were not significantly different for miners without CWP versus miners with CWP. On the other hand, oxidative DNA-danage was significantly lower in the non-dust-exposed control group versus reference miners. Although age in the control group was significantly higher, correlation between age and oxidative DNA-damage was observed neither in any of the subgroups nor in the total study group. The calculated cumulative exposure (to respirable coal dust as well as quartz content) was significantly higher in the miners with CWP compared to reference miners, but years exposed, time elapsed since first exposure and time retired were not (Table 6.1). However, none of the exposure parameters was related to oxidative DNA damage in a stepwise multiple linear regression. No relation was observed between stage of pneumoconiosis and oxidative DNA damage (data not shown), nor was oxidative DNA-damage affected by medication.

Table 6.1 Study group characteristics and ratios of 8oxodG to dG in peripheral blood lymphocytes of miners with coal workers pneumoconiosis, reference miners and a non-dust-exposed control group.

\begin{tabular}{|c|c|c|c|}
\hline \multirow[b]{3}{*}{ age (years) } & \multirow{2}{*}{$\begin{array}{c}\text { non-exposed controls } \\
\mathrm{n}=24\end{array}$} & reference miners & miners with CWP \\
\hline & & $n=30$ & $\mathbf{n}=8$ \\
\hline & $50.7(1.1)$ & $46.6(0.9) \#$ & $46.5 \quad(2.1)$ \\
\hline smoking (pack-years) & $105.6(18.6)$ & $92.9 \quad(16.4)$ & $140.4 \quad(37.9)$ \\
\hline years underground & - & $22.1 \quad(0.8)$ & $24.6(1.7)$ \\
\hline years since first exposure & - & 28.1 & $29.1 \quad(1.9)$ \\
\hline months retired & - & $44.4 \quad(3.4)$ & $51.5 \quad(2.7)$ \\
\hline cumulative exposure & & & \\
\hline $\operatorname{dust}\left(\mathrm{ghm}^{-3}\right)$ & - & $92.6 \quad(11.7)$ & $144.9(11,8)^{*}$ \\
\hline quartz $\left(\mathrm{ghm}^{-3}\right)$ & - & $4.90 \quad(0.56)$ & $7.42(0.61)^{*}$ \\
\hline 8-oxodG/dG $\left(\times 10^{-5}\right)$ & $1.67 \quad(0.27)$ & $2.96 \quad(0.34) \#$ & $2.61 \quad(0.44)$ \\
\hline
\end{tabular}

All values (i.e. mean and standard error) are evaluated by the Mann-Whitney $U$ test: "p $<0.05$ significantly different from reference miners; \#p<0.01 significantly different from non-dust-exposed controls; CWP $=$ miners with coal workers 'pneumoconiosis ( $\mathrm{LO}$ chest radiograph $>0 / 0$ ).

No differences in amount of smoking were present among the three groups, and no correlation was observed between 8 -oxodG/dG ratios and pack-years in any of the subgroups or in the total group. The effects smoking on the 8-oxodG/dG ratios are also shown in Table 6.2. Oxidative DNA-damage did not differ between current smokers and nonsmokers, or between former smokers and never smokers. No synergistic or antagonistic effects of smoking plus dust exposure on 
8-oxodG/d(; ratios were observed (Table 6.2). Multiple regression (stepwise analysis) did not result in any significant relation between any parameter of smoking, exposure, age or medication and the ratio of 8-oxodG/dG.

Table 6.2 Effects of smoking on the ratio of 8-oxodG $/ \mathrm{dG}\left(\times 10^{-5}\right)$ in peripheral blood lymphocytes of controls and coal miners.

\begin{tabular}{lcccc} 
& $\begin{array}{c}\text { non-exposed } \\
\text { controls }(\mathrm{n}=24)\end{array}$ & $\begin{array}{c}\text { reference } \\
\text { miners }(\mathrm{n}=30)\end{array}$ & $\begin{array}{c}\text { miners with } \\
\text { CWP }(\mathrm{n}=8)\end{array}$ & $\begin{array}{c}\text { all subjects } \\
(\mathrm{n}=62)\end{array}$ \\
\hline smokers & $1.31(0.28)$ & $2.86(0.65)$ & $3.14(0.85)$ & $2.26(0.36)$ \\
non smokers & $(n=11)$ & $(n=10)$ & $(n=4)$ & $(n=25)$ \\
& $1.96(0.44)$ & $3.01(0.40)$ & $2.07(0.11)$ & $2.54(0.28)$ \\
ever smokers & $(n=1.3)$ & $(n=20)$ & $(n=4)$ & $(n=37)$ \\
& $1.55(0.20)$ & $3.02(0.40)$ & $2.71(0.50)$ & $2.40(0.23)$ \\
never smokers & $(n=20)$ & $(n=24)$ & $(n=7)$ & $(n=51)$ \\
& $2.23(1.37)$ & $2.72(0.57)$ & $1.87(-)$ & $2.47(0.55)$ \\
& $(n=4)$ & $(n=6)$ & $(n=1)$ & $(n=11)$
\end{tabular}

All values (i.e. mean and standard error) are evaluated by the Mann-Whitney $U$ test at $p=0.05$; $\mathrm{CWP}=$ Coal workers 'pneumoconiosis (ILO chest radiograph $>0 / 0$ ).

\section{Discussion}

In the present study we evaluated the ratio of 8 -oxodG to $\mathrm{dG}$ residues in peripheral blood lymphocytes of coal workers, and controls not occupationally exposed to (mineral) dusts. Oxidative DNA damage was found to be significantly higher in lymphocytes of coal miners without pneumoconiosis than on those of the non-exposed controls. A similar study by Hanaoka et al. [1993] studying 8oxodG/dG ratios in lymphocytes of asbestosis patients did not identify differences with subjects without asbestosis. However, their control group was selected from patients from the same hospital. Moreover, all asbestosis patients studied by Hanaoka et al. [1993] had radiological evidence of fibrosis (chest radiograph findings of $1 / 0-2 / 1$ according to ILO criteria [ $\mathrm{LO}, 1980]$ ). In contrast, the majority of the coal workers in our present study (30/38) were not fibrosis patients. In our study lymphocyte 8-oxodG/dG ratios were not related to smoking habits, age or medication, nor was there a sinergistic effect between smoking and occupational exposure. This indicates that the chronic inhalation of mineral dust is the major factor responsible for the observed difference in oxidative DNA damage between miners and controls. However, individual 8-oxodG/dG ratios of coal miners did not correlate with cumulative exposure estimates or to the presence of fibrotic 
lesions visible by chest radiography. Therefore, we suggest that either the presence or absence of a history of long term heavily occupational exposure to mineral dusts may account for a all-or-none significant contribution to lymphocyte DNA damage.

Several authors have shown that mineral dusts are able to increase oxidative damage in (isolated) DNA [Leanderson et al. 1988; Adachi et al, 1994; Takeuchi and Morimoto 1994]. Recently, Daniel et al. [1994] showed that silica can induce strand breaks in isolated DNA. Effects of silica have been described on sister chromatid exchanges [Pairon et al. 1990], in cell transformation assays and micronucleus assay [Hesterberg et al. 1986]. Interestingly, Pairon et al. showed that sister chromatid exchange frequencies in lymphocytes were invreased upon exposure to tridymite only if monocytes were also present in the culture dishes [Pairon et al. 1990]. Although less clear results were observed with silica, the authors suggested that a phagocytotic action of phagocytotic cells (monocytes) and the subsequent signaling mechanisms between these cells and the target cells (lymphocytes) are necessary to induce cytogenetic effects. Similarly, signaling between pulmonary cells (macrophages, epithelial cells, fibroblasts) in the presence of coal dust and peripheral blood lymphocytes may be the reason for the increased 8-oxodG levels observed in the lymphocytes of the miners. Recently, Driscoll et al. [1994] have presented in vivo support for a mutagenic action of silica. They showed that upon silica inhalation, rat alveolar epithelial cells have increased mutagenicity (HPRT locus mutations), and even more interestingly, that cultured rat epithelial cells also show an increased mutagenic response upon incubation with bronchoalveolar lavage cells from these silica-exposed rats [Driscoll et al. 1994]. However, in contrast to studies on direct interactions between mineral dust and DNA [Leanderson et al. 1988; Adachi et al, 1994; Janssen et al. 1994b], it is not clear whether or not ROS and antioxidant status play a significant role in the indirect genotoxic action of silica observed by Pairon et al. [1990] and Driscoll et al. [1994].

Previously, we have reported that antioxidant factors in the blood may be changed by chronic silica exposure or by the presence of silicotic lesions [Borm et al. 1986; Engelen et al. 1988; Evelo et al. 1993; Schins et al. 1994a]. Unfortunately, we have no data on antioxidant protection of lymphocytes in these workers, but neither red blood cell glutathione, glutathione-S-transferase and glutathione reductase, nor plasma total radical trapping capacity $(n=13)$ [Schins et al. 1994a] were related to oxidative DNA damage (data not shown). However, our data do show that peripheral blood cells in (healthy) coal miners with a long working history [Borm et al. 1986; Borm et al. 1990; Schins et al. 1994c] behave and respond differently in comparison to cells of subjects without a mining history. We suggest that oxidative damage to the DNA of peripheral blood lymphocytes may be introduced by increased oxidative stress responses in the lung in subjects 
chronically exposed to mineral dusts. Whether this is an important pathway in the suggested carcinogenicity of silica is still an open question.

Acknowledgements. The authors thank all coal miners and the Kempense Steenkoolmijnen NV for their willingness to cooperate in this study; Marc van Sprundel, Luc Lenaerts, and Luc Mariën for reading the X-rays; Bernard Préat for the calculation of individual cumulative exposure; Angelique Vankan, Jolanda van Golde and Thim Derhaag for technical assistance; Jan Lutgerink for his advice with regard to DNA extractions. This study was supported by grant no. 7263/03/092 of the European Community for Steel \& Coal. 


\title{
Chapter 7
}

\section{BLOOD ANTIOXIDANT STATUS IN COAL DUST INDUCED RESPIRATORY DISORDERS: A LONGITUDINAL EVALUATION OF MULTIPLE BIOMARKERS.}

\author{
Roel PF Schins, Soedjajadi Keman, Paul JA Borm. \\ Department of Health Risk Analysis and Toxicology, University of Limburg, \\ PO BOX 616, $6200 \mathrm{MD}$, Maastricht, the Netherlands.
}

Accepted for publication in: Bionarkers.

\begin{abstract}
To investigate the involvement of oxidative stress in coal dust induced respiratory disorders, red blood cell and serum antioxidants in 66 coal miners were related to fiveyear changes in coal workers pneumoconiosis (CWP), chronic bronchitis, and lung function decrease $(n=40)$. Reduced (GSH) and oxidized (GSSG) glutathione concentrations, glutathione peroxidase (Gpx), glutathione-S-transferase (GST), superoxide dismutase (SOD), and catalase activities were measured in erythrocytes and Vitamin A, Vitamin E and iron were determined in serum. Changes in CWP were determined by chest radiography, chronic bronchitis was determined from a validated questionnaire and lung function decline was calculated by linear regression for a ten year interval before blood sampling. SOD activity was increased in miners with progression of CWP $(2308 \pm 156$ vs $1703 \pm 155 \mathrm{U} / \mathrm{gHb}, \mathrm{p}<0.05)$, and GSH was reduced in those with chronic bronchitis at follow-up $(3.53 \pm 0.16$ vs $4.05 \pm 0.09 \mathrm{mmole} / \mathrm{gHb}, \mathrm{p}<0.01)$. Stepwise discriminant analysis showed that for both pneumoconiotic and nonpneumoconiotic respiratory disease in this cohort, increased enzymatic antioxidants (i.e. Gpx, Catalase, SOD) were high risk factors, while increased "nonenzymatic" antioxidants (i.e. Vitamin E, GSH) indicated reduced risk. GST activity showed discriminative power in two ways, i.e. decreased activity in those at risk for CWP, but increased in those at risk to obstructive disease. We conclude that the multiple marker approach applied here, shows the relevance of interpretation of total "antioxidant status" versus single antioxidant measurements in health screening of individuals at risk for respiratory impairments.
\end{abstract}

\section{Introduction}

It is well known that occupational exposure to coal dust can lead to coal workers' pneumoconiosis [Crystal et al. 1991]. However, chronic inhalation of coal dust 
may also cause other respiratory effects such as emphysema, chronic bronchitis and airflow obstruction [Wouters et al. 1994]. The role of oxygen radicals in these diseases has been discussed extensively over the last decade [Cantin and Crystal 1985, Barnes 1990, Kehrer 1993, Halliwell and Cross 1994]. Because of the intrinsic physicochemical characteristics of mineral dusts and their complex behaviour in the lung, the significance of "oxidative stress" in the development of mineral dust related respiratory disorders has received special attention [Kamp et al. 1992, Janssen et al. 1992]. Previously, we showed that many components of the blood antioxidant system of coal miners were related to the severity of coal workers pneumoconiosis (CWP) [Engelen et al. 1990]. More recently, altered antioxidant status in coal dust exposure and stage of pneumoconiosis was also reported by others, in the blood [Perrin-Nadif et al. 1996], and in bronchoalveolar lavage [Vallyathan et al. 1995]. Since antioxidant status has also been related to obstructive disease [Morabia et al. 1989, Schwartz and Weiss 1994, Britton et al. 1995], one could suggest that the impaired oxidant/antioxidant balance observed in coal workers [Engelen et al. 1990] may also play a role in the nonpneumoconiotic respiratory effects in these subjects.

Although the presence of "oxidative stress" in coal dust exposed subjects may be evident, its significance in the development or progression of coal dust induced respiratory disorders remains to be elucidated. To determine the significance of antioxidant measurements with regard to the development or progression of a disease, a longitudinal design is necessary [Schulte et al. 1993]. However, follow-up studies dealing with the prognostic power of antioxidant status are scarce. In the present study several blood antioxidant parameters previously measured in coal workers are related to prospective changes in pneumoconiosis (chest radiography) and the presence of chronic bronchitis, as well as to retrospective analysis of lung function decline. Aim of this study is to determine the relevance of blood antioxidant status in surveillance and screening of (retired) coal workers. For this purpose discriminant analysis is used to determine to what extent multiple blood antioxidants as indicators of "oxidative stress", may identify coal workers at increased risk for coal dust induced respiratory disorders.

\section{Methods}

\section{Study design}

Figure 7.1 shows the design of the study. Blood antioxidant parameters, evaluated in red blood cells or in the plasma of 91 coal workers were related to five-year changes in chest radiographs and to 10-year lung function decline. Reduced (GSH) and oxidized (GSSG) glutathione concentrations, total (Gpx-To) and selenium dependent (Gpx-Se) glutathione peroxidase activities, glutathione- 
S-transferase activity (GST), superoxide dismutase activity (SOD) and catalase activity were measured in red blood cells and concentrations of Vitamin A, Vitamin $\mathrm{E}$ and iron $(\mathrm{Fe}$ ) were determined in serum. GST levels were measured only in 64 subjects of this cohort [Evelo et al. 1993]. All subjects previously screened for these parameters were recruited for a five-year follow-up and upon written informed consent, a new chest radiograph was made. A questionnaire was combined with a personal interview to determine job-history, smoking and medical status. Furthermore, all medical files were screened retrospective for a 10-year period to determine longitudinal lung function decline (see Figure 7.1).

Figure 7.1. Design of the study.

Antioxidants measured in red blood cells or plasma of coal workers were related to lung function decline over a 10-year interval (retrospective), and to development or progression of CWP, and chronic bronchitis determined at 5-year follow-up (prospective). Lung function decline was determined by longitudinal analysis of spirometry data (FEV1 and FVC) determined between 1987 and 1992. Progression of CWP was evaluated by paired comparison of chest-radiographs (CR) made in 1987 and 1992. Job-history (including calculation of cumulative dust exposure), smokin medication and medical history (including chronic bronchitis) were determined at follow-up from questionnaires, personal interviews and

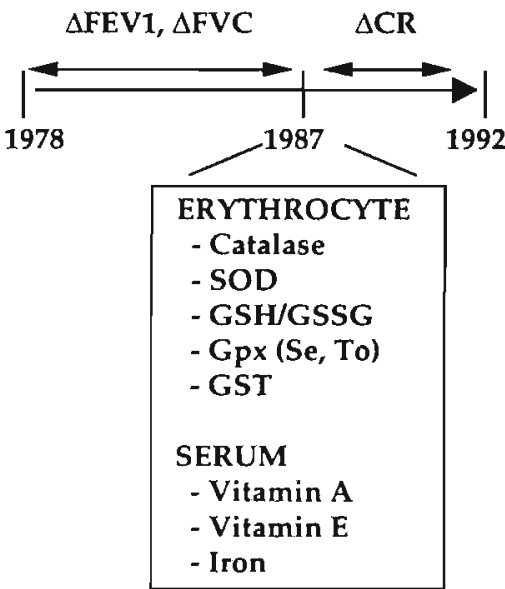
medical files (see text for details).

Analysis of blood antioxidant parameters

Blood sampling and treatment, and analysis of red cell and plasma antioxidants was done as described previously [Engelen et al. 1990, Evelo et al. 1993]. Briefly, $\mathrm{Hb}$ concentration in red blood cells was measured spectrophotometrically.

Plasma iron concentrations were determined by the Iron Ferro-Zine test of Roche Diagnostica [Stookey, 1970]. Antioxidant activities in erythrocytes were determined spectrophotometrically, according to the methods of Maral et al. [1977] (Gpx), McCord and Fridovich [1969] (SOD), Aebi [1984] (Catalase), and Habig and Jacoby [1981] (GST). GSH and GSSG concentrations were determined according to the protocol described by Hissin and Hilf [1976]. Vitamin A and E were simultaneously measured by HPLC according to Cuesta Sanz and SantaCruz [1986]. 


\section{Che'st raliography and lung function}

Chest radiographs taken in 1987 and in 1992 were scored according to the classification rules of the International Labour Organisation [ILO 1980] by an experienced panel of three physicians. Scoring was done in plenary sessions where consensus had to be reached based on individual scores. Five-year progression or new development of CWP was determined from a paired reading session of each individuals' chest radiograph of 1987 and 1992 [Schins et al. 1994].

Lung function decline $\left(\triangle F E V_{1}\right.$ and $\left.\triangle F V C\right)$ was calculated by linear regression for a ten year interval before blood sampling (1978 - 1987). Lung function had been measured periodically at medical screening using a Vitalograph TM spirometer (Vitalograph Ltd, UK) at two mine-pits and a Vicatest 4 (Mijnhardt, Odijk, Holland). Tiffeneau index was calculated from $\mathrm{FEV}_{1}$ divided by FVC times $100 \%$. Lung function decline of coal miners was calculated as the regression slope obtained with least-square analysis through minimally 8 out of 10 yearly measurements. Lower and upper quartiles of $\triangle \mathrm{FEV}_{1}$ were used as cut off point to define rapid versus slow decliners.

\section{Exposure, smoking, medication, and medical history}

Occupational history from each subject was gathered from the personel and medical files. Individual dust exposure was calculated from each individuals' job exposure matrix obtained from a personal interview at follow-up and the medical file. Exposure parameters used for this study were: (I) cumulative dust exposure, (II) cumulative quartz exposure, (III) years underground, and (IV) years underground. Cumulative exposure was calculated as described elsewhere [Schins and Borm., 1995].

A validated questionnaire [Cotes et al. 1987] was sent to all participants in which medical symptoms, medical history, smoking habits and medication were asked. Answers were verified during personal interviews and by comparison with records from the preceding study [Engelen et al. 1990]. Smoking amounts (pack years) were calculated as described elsewhere [Jorna et al. 1994]. The questionnaire was used to identify respiratory symptoms at follow-up. When bronchitis-like symptoms (i.e. presence of both cough and pleghm) were reported for most days of thee week and at least three months per year, the subject was classified as "chronic bronchitis".

\section{Statistical evaluations}

Correlations between antioxidant levels and (cumulative) exposure, age, smoking, lung function were evaluated by Spearman Rank correlation. Unpaired differences between 1987 and 1992 (sub) groups were tested by the Mann Whitney U-test. Differences between miners without or with CWP progression, obstruction, and respiratory symptoms were evaluated by the MannWhitney test. Finally, stepwise discriminant analysis was used to determine 
whether each individuals' "antioxidant status", i.e. the use of multiple antioxidant parameters would allow classification of a coal miners into mutually exclusive groups [Dillon and Goldstein 1984]. Therefore, all subjects were classified in the following groups: Subjects (1) with or without development of CWP, (2) with or without progression of already existing CWP, subjects with (3) slow or rapid longitudinal FEV1-decline, and (4) subjects with or without chronic bronchitis. Statistical evaluations were made using SPSS 6.1 (Inc. Chicago, IL).

\section{Results}

Ultimately 66 out of 91 coal miners previously screened for antioxidant parameters (73\% of the original cohort) participated in our follow-up. GST levels were only known for 46 subjects of this group. Lung function decline (retrospective analysis of medical files) was obtained from 40 out of 91 subjects. With regard to these subgroups, no selection bias was observed for age, smoke habits, exposure, medication, pneumoconiotic stage and antioxidants (data not shown). None of the antioxidant levels listed in Table 7.1 was significantly related to individual cumulative (dust/quartz) exposure nor to any other exposure estimate (i.e. years since first exposure or years exposed). Furthermore, no antioxidant levels was significantly related to age or smoking habits (pack years).

\section{Single marker analysis}

Based on the paired chest radiograph reading sessions, it was observed that three out of 52 miners newly developed CWP, while 8 out of 14 miners had progression of already existing CWP. None of the initial antioxidant data of these subgroups (Table 7.1) was significantly different for miners with or without disease development or progression, only red cell SOD activity was significantly higher in miners with subsequent progression of CWP $(\mathrm{p}<0.05)$. As can be seen in Table 7.1, this finding was not biased by differences in distributions of age, smoking or (cumulative) exposure. In the miners without development of CWP, one subject was identified with red blood cell catalase deficiency. Statistical analysis was repeated upon exclusion of this subject (see legend of Table 7.1).

The average decline in $\mathrm{FEV}_{1}$ was $-34.1 \mathrm{~mL} /$ year $(\mathrm{n}=40)$, and based on upper and lower quartiles subjects were divided into rapid (cut off $-68.4 \mathrm{~mL} /$ year) or slow decliners (cut off $-6.9 \mathrm{~mL} /$ year). However, no significant correlations were observed between longitudinal lung function decline $\left(\triangle F E V_{1}\right.$ or $\triangle F V C$ ) and individual antioxidant parameters, nor was any significant difference seen between slow and rapid decliners for any antioxidant (see Table 7.2). FEV - $^{-}$ decline was not significantly different between subjects with or without progression of pneumoconiosis (see Table 7.1). 
Table 7.1 Blood antioxidant parameters, age, exposure, smoking and the 5-year progression of pneumoconiosis in the coal workers.

\begin{tabular}{|c|c|c|c|c|}
\hline & \multicolumn{2}{|c|}{ Reference miners } & \multicolumn{2}{|c|}{ Miners with CWP } \\
\hline & $\begin{array}{c}\text { NP } \\
(n=49)\end{array}$ & $\begin{array}{c}P \\
(n=3)\end{array}$ & $\begin{array}{c}N P \\
(n=6)\end{array}$ & $\begin{array}{c}P \\
(n=8)\end{array}$ \\
\hline Age, years (in 1992) & $47.6(0.7)$ & $45.3(3.8)$ & $48.6(1.4)$ & $52.6(1.3)$ \\
\hline Underground in 1987, years & $21.8(0.6)$ & $23.0(4.6)$ & $24.2(1.1)$ & $26.0(1.1)$ \\
\hline Total underground (1992), years & $22.9(0.6)$ & $24.0(4.0)$ & $25.0(1.4)$ & $26.6(1.4)$ \\
\hline Cumulative dust, gh/m3 (in 1992) & $95(8)$ & $135(33)$ & $141(4)$ & $123(25)$ \\
\hline Smoking, packyears (in 1992) & $124(17)$ & $143(34)$ & $198(71)$ & $190(45)$ \\
\hline FEV1-decline (mL/year) ${ }^{a}$ & $-30.1(9.9)$ & $-69.8(43.2)$ & $-36.1(14.3)$ & $-82.1(41.5)$ \\
\hline Chronic bronchitis, yes / no b & $8 / 41$ & $1 / 2$ & $1 / 5$ & $2 / 6$ \\
\hline $\mathrm{Hb}, \mathrm{mg} \mathrm{Hb} / \mathrm{mL}$ & $15.1(0.28)$ & $13.9(0.33)$ & $14.4(0.31)$ & $15.8(0.46)$ \\
\hline $\mathrm{SOD}, \mathrm{U} / \mathrm{gHb}$ & $1979(74)$ & $1740(190)$ & $1703(156)$ & $2308(155)^{*}$ \\
\hline Catalase, $\mu$ mole $\mathrm{H} 2 \mathrm{O} 2 / \mathrm{min} / \mathrm{g} \mathrm{Hb}^{c}$ & $0.45(0.01)$ & $0.50(0.04)$ & $0.43(0.02)$ & $0.46(0.02)$ \\
\hline GSHpx-To, $\mu$ mole NADPH $/ \mathrm{min} / \mathrm{g} \mathrm{Hb}$ & $9.67(0.09)$ & $9.79(0.15)$ & $9.64(0.20)$ & $9.77(0.13)$ \\
\hline GSHpx-Se, $\mu$ mole NADPH $/ \mathrm{min} / \mathrm{g} \mathrm{Hb}$ & $9.25(0.11)$ & $9.39(0.28)$ & $9.20(0.33)$ & $9.39(0.16)$ \\
\hline GST, U/gHbd & $3.11(0.23)$ & $2.35(0.50)$ & $3.63(0.42)$ & $2.54(0.34)$ \\
\hline GSH, mmole/g Hb & $3.99(0.09)$ & $3.38(0.42)$ & $3.92(0.25)$ & $3.84(0.20)$ \\
\hline GSSG, $\mu$ mole $/ \mathrm{g} \mathrm{Hb}$ & $.036(.001)$ & $.033(.004)$ & $.037(.003)$ & $.035(.002)$ \\
\hline $\mathrm{Fe}, \mu \mathrm{mole} / \mathrm{mL}$ plasma & $18.3(1.1)$ & $21.0(5.8)$ & $18.5(1.9)$ & $14.7(1.3)$ \\
\hline Vitamin $\mathrm{A}, \mu \mathrm{g} / \mathrm{mL}$ plasma & $9.4(0.4)$ & $7.9(0.6)$ & $11.2(1.3)$ & $8.9(0.9)$ \\
\hline Vitamin E, $\mu \mathrm{g} / \mathrm{mL}$. plasma & $14.6(0.7)$ & $11.7(2.8)$ & $20.9(4.3)$ & $14.0(1.7)$ \\
\hline
\end{tabular}

P, pregression (or development) of pneumoconiosis; NP, no progression of CWP; The asterisk (*) denotes a significant difference from NP, $p<0.05$, Mann-Whitney U-test.

a FEV1-decline was obtained from 35 miners (i.e. $n=26, n=2, n=4, n=3$ subjects respectively); ${ }^{b}$ Odds ratios for chronic bronchitis and progression were 2.6 in references and 1.7 in CWP group, both not significant at $90 \%$ C.I.; ${ }^{C}$ Upon exclusion of one catalase deficient subject, average of NP references becomes $0.46(0.01)$; $d$ GST was related to progression in 46 miners (i.e. $n=33, n=2, n=6, n=5$ subjects respectively).

Based on the questionnaires, 18 and 12 subjects were classified having bronchitis or chronic bronchitis at follow-up. Both development or progression of CWP were independent from the presence of (chronic) bronchitis (see Table 7.1), and FEV 1 -decline was not different between subjects with or without chronic bronchitis (see Table 7.2). Interestingly, in subjects with chronic bronchitisred cell GSH concentration was significantly reduced compared to normals $(\mathrm{p}<0.01)$. 
Table 7.2 Blood antioxidant parameters, age, exposure, smoking and the 10-year retrospective FEV1-decline in the coal workers.

\begin{tabular}{|c|c|c|c|c|}
\hline & \multicolumn{2}{|c|}{ FEV1-decline } & \multicolumn{2}{|c|}{ Chronic bronchitis } \\
\hline & $\begin{array}{l}\text { high } \\
(n=10)\end{array}$ & $\begin{array}{c}\text { low } \\
(n=10)\end{array}$ & $\begin{array}{c}\text { Yes } \\
(n=12)\end{array}$ & $\begin{array}{c}\text { No } \\
(n=53)\end{array}$ \\
\hline Age, years (in 1992) & $48.2(1.7)$ & $46.1(1.2)$ & $49.5(1.6)$ & $47.9(0.6)$ \\
\hline Underground in 1987 , years & $23.0(3.9)$ & $22.1(1.3)$ & $23.2(1.3)$ & $22.5(0.6)$ \\
\hline Total underground (1992), years & $23.8(1.0)$ & $23.5(1.4)$ & $23.8(1.3)$ & $23.6(0.6)$ \\
\hline Cumulative dust, gh/m3 (in 1992) & $100(19)$ & $93(18)$ & $89(17)$ & $108(8)$ \\
\hline Smoking, packyears (in 1992) & $199(51)$ & $136(35)$ & $161(38)$ & $135(17)$ \\
\hline FEV1-decline (mL/year) ${ }^{a}$ & $-100.4(9.5)$ & $17.0(10.0)^{* *}$ & $-10.1(29.3)$ & $-44.6(7.8)$ \\
\hline Chronic bronchitis, yes / no $b$ & $1 / 9$ & $2 / 8$ & & \\
\hline $\mathrm{Hb}$, $\mathrm{mg} \mathrm{Hb} / \mathrm{mL}$ & $15.8(0.6)$ & $14.7(0.6)$ & $15.2(0.5)$ & $15.0(0.2)$ \\
\hline $\mathrm{SOD}, \mathrm{U} / \mathrm{gH} \mathrm{b}$ & $2079(287)$ & $1989(128)$ & $2171(126)$ & $1941(71)$ \\
\hline Catalase, $\mu$ mole $\mathrm{H} 2 \mathrm{O} 2 / \mathrm{min} / \mathrm{g} \mathrm{Hb}$ & $0.46(0.02)$ & $0.43(0.01)$ & $0.43(.01)$ & $0.47(0.01)$ \\
\hline GSHpx-To, $\mu$ mole NADPH/min/g Hb & $9.86(0.12)$ & $9.48(0.29)$ & $9.78(0.16)$ & $9.65(0.08)$ \\
\hline GSHpx-Se, $\mu$ mole NADPH/min/g Hb & $9.35(0.13)$ & $9.22(0.27)$ & $9.36(0.21)$ & $9.24(0.10)$ \\
\hline $\mathrm{GST}, \mathrm{U} / \mathrm{gHb}^{\mathrm{c}}$ & $3.09(0.27)$ & $2.87(0.57)$ & $3.32(0.27)$ & $2.99(0.21)$ \\
\hline $\mathrm{GSH}, \mathrm{mmole} / \mathrm{g} \mathrm{Hb}$ & $4.00(0.18)$ & $4.12(0.15)$ & $3.53(0.16)$ & $4.05(0.09)^{* *}$ \\
\hline GSSG, $\mu$ mole / g Hb & $.035(.003)$ & $.038(.003)$ & $0.035(.003)$ & $0.035(.001)$ \\
\hline Fe, $\mu$ mole $/ \mathrm{mL}$ plasma & $16.9(1.3)$ & $18.1(2.0)$ & $18.7(3.5)$ & $18.0(1.0)$ \\
\hline Vitamin $\mathrm{A}, \mu \mathrm{g} / \mathrm{mL}$ plasma & $10.5(1.3)$ & $10.6(1.2)$ & $10.6(0.9)$ & $9.2(0.4)$ \\
\hline Vitamin $\mathrm{E}, \mu \mathrm{g} / \mathrm{mL}$ plasma & $16.6(2.3)$ & $16.7(1.8)$ & $17.7(1.7)$ & $14.5(0.8)$ \\
\hline
\end{tabular}

${ }^{* *} \mathrm{p}<0.01$ (Mann-Whitney U-test); The catalase deficient subject was excluded from analysis. a FEV1-decline determined in $n=10, n=10, n=5, n=35$ subjects respectively; ${ }^{b}$ Odds ratio $=0.44$, for chronic bronchitis vs. lung function decline; not siginificant at $90 \%$ C.I.; ${ }^{c}$ GST subgroup numbers are 7 vs 9 , and 7 vs 38 respectively.

\section{Multiple marker analysis}

To determine the involvement of multiple antioxidants in specific disease a discriminant analysis was performed. Due to the distribution skewness of several antioxidant parameters, analysis was performed on logarithm-transformed data. Antioxidants tested in the discriminant function were: Catalase, SOD, GSH (i.e. reduced glutathione only), GST, Gpx (i.e. total), Fe, Vitamin A and Vitamin E. Missing values (for GST, Vitamin A and Vitamin E) were replaced with the (Intransformed) group average. Furthermore, a catalase "outlier" subject was excluded from all multiple statistical analyses. The results of the stepwise 
analysis are shown in Table 7.3, for several discriminating criteria along with the percentage of correct grouped subjects and its significance $\left(\chi^{2}\right)$, and the antioxidants significantly attributing to the correct grouping. $86 \%$ of the subjects were correctly grouped into progression $(n=8)$ or no progression $(n=6)$ of already existing CWP, when erythrocyte GSH concentration, SOD and GST activities, and serum Vitamin $E$ status were included in the discriminant function $\left(\mathrm{d} f=4, \chi^{2}\right.$ $=14.2, p<0.01$ ). The discriminant function indicated a negative association with disease progression for GSH, Vitamin E and GST, while SOD was higher in those with progression. Furthermore, a significant discrimination of subjects classified according to new development of CWP (3 versus 48 subjects) was observed with GSH, Vitamin (both negative) and catalase and iron (positive) in the discriminant model. A discrimination between slow and rapid decliners in FEV 1 was obtained with SOD, catalase, glutathione-S-transferase and glutathione peroxidase in the model. Interestingly, these parameters were all increased in those with rapid decline in FEV 1 in comparison with those with a low decline. The presence or absence of chronic bronchitis was significantly discriminated with GSH, SOD and Vitamin A, i.e. explained by lower GSH and increased SOD and Vitamin A levels in the symptomatic group.

Table 7.3 Discriminant analysis of antioxidants in erythrocytes and plasma in relation to coal dust induced respiratory disorders

\begin{tabular}{|c|c|c|c|c|c|c|}
\hline $\begin{array}{l}\text { Classification } \\
\text { criterium }\end{array}$ & yes/no & $\begin{array}{c}\text { Grouped } \\
(\%)\end{array}$ & $\chi^{2}$ & & p-value & $\begin{array}{l}\text { Variables included in } \\
\text { discrimant model }\end{array}$ \\
\hline Development of CWP & $3 / 48$ & 86.0 & 12.3 & 4 & $<0.02$ & GSH (-), VitE $(-)$, Fe $(+)$, Cat $(+)$ \\
\hline Progression of CWP & $8 / 6$ & 85.7 & 14.2 & 4 & $<0.01$ & $\operatorname{GSH}(-), \operatorname{VitE}(-), \operatorname{GST}(-), \operatorname{SOD}(+)$ \\
\hline Lung function decline & $10 / 10$ & 85.7 & 10.2 & 4 & $<0.04$ & $\operatorname{Gpx}(+), \operatorname{SOD}(+), \operatorname{GST}(+)$, Cat $(+)$ \\
\hline Chronic bronchitis & $12 / 53$ & 70.3 & 12.6 & 3 & $<0.01$ & GSH (-), SOD (+), VitA (+) \\
\hline
\end{tabular}

\# All subjects were classified as follows: (1) presence or absence of development of CWP; (2) presence or absence progression of already existing CWP; (3) rapid (= "yes") or slow (= "no") decline in lung function (i.e lower versus upper quartile of $\triangle \mathrm{FEV}_{1}$ )

Stepwise discriminant analysis was performed on natural logarithm-transformed data. Antioxidants tested in the discriminant function were: Catalase, SOD, GSH, GST, Gpx (total), Fe, Vitamin A and Vitamin E. Missing values were replaced with the group average. For each classification factor, the Table shows the percentage of correct grouped subjects, the significance of the canonical correlation and the antioxidants significantly attributing to the correct grouping, i.e. positive $(+)$ or negative $(-)$ in the canonical correlation function. 


\section{Discussion}

As stated by Halliwell and Cross [1994], the major issue is not whether oxidative stress can be demonstrated in a disease, but whether it makes a significant contribution to the disease activity. Previously, we and others elucidated the first question for subjects chronically exposed to coal dust by showing different antioxidant status in stages of coal workers pneumoconiosis [Engelen et al. 1990, Evelo et al. 1993, Perrin-Nadif et al. 1996]. Glutathione concentrations as well as glutathione-S-transferase activities were significantly decreased at early stage pneumoconiosis, while at later stages erythrocyte GSH and GST were back to normal [Engelen et al. 1990, Evelo et al. 1993]. In these cross-sectional studies, our original hypothesis was that subjects who develop pneumoconiosis are "less well equipped to deal with reactive oxygen species" [Engelen et al. 1990], and that erythrocytes could be considered as important circulating antioxidant carriers in this disease. Further support for this hypothesis is provided by our recent observation that oxidative DNA damage (i.e. 8-hydroxydeoxyguanosine residues) is increased in peripheral blood lymphocytes of coal workers compared to nonexposed controls [Schins et al. 1995]. However, whether the variation in oxidative stress observed in coal workers significantly attributes to respiratory disease, would necessitate a different experimental approach.

In the present study, prospective analysis of the above cohort since 1987 has shown that red blood cell SOD activity was significantly higher in miners with subsequent progression of pneumoconiosis as compared to those without progression at five-year follow-up. Previously we reported that SOD activities were not increased in coal workers with pneumoconiosis compared to healthy miners [Engelen et al. 1990]. However, increased erythrocyte SOD levels were found recently in underground coal miners compared to surface workers [PerrinNadif et al. 1996]. This might be a consequence of differences in coal dust exposures but one could also consider these findings to reflect the higher pneumoconiosis risk for underground versus surface workers. Previously, it has been shown that macrophage superoxide anion $\left(\mathrm{O}_{2}{ }^{\circ-}\right)$ generation was increased in coal workers compared to non-exposed controls [Rom et al. 1987, Wallaert et al. 1990], and the activity of SOD may therefore reflect the extent of a respiratory $\mathrm{O}_{2}{ }^{\bullet-}$ burst from macrophages and neutrophils in the lung. This is underscored by animal data, demonstrating a direct upregulation of manganese SOD expression and inmmunoreactive protein in the lung by mineral dusts, which was related to the inflammatory response [Janssen et al. 1992].

Apart from SOD, also other antioxidants were consistently different between subgroups although sometimes only on borderline significance. A combination of antioxidant parameters tested by discriminant analysis, showed that in addition to SOD, also GST, GSH and Vitamin E status denominated presence or absence of CWP progression. However, in contrast to SOD activity, these 
parameters were reduced in those with progression (see also Table 7.1). Interestingly, Vitamin $\mathrm{E}$ and GSH were also reduced in the few $(n=3)$ healthy miners who developed pneumoconiosis during follow-up, while in those subjects catalase activity and plasma iron were increased. Therefore, reduced levels of red cell glutathione and plasma Vitamin E status may be considered as risk factors for CWP. The concept of reduced GSH as a possible fibrotic risk factor, was also discussed by Cantin et al. who showed that reduced extracellular GSH was associated to increased lung fibroblast proliferation [Cantin et al. 1990]. The significance of reduced GST in fibrosis may be closely related to this observation. Its reduced activity may be related to oxidative stress during lipid peroxidation [Halliwell and Cross 1994], which is considered as an important feature of mineral dust related lung toxicity. Another support for the involvement of GST in fibrosis comes from Smith et al. [1994], who recently showed that GST- $\mu$ class (GST-M1) deficient subjects were at increased risk for asbestosis. Although the majority of GST activity in erythrocytes originates from GST-P class isoenzymes, GST polymorphism could, in addition to its role in (smoking related) lung cancer [Seidegard et al. 1986, 1990], be significantly involved in the development of pneumoconiosis. Vitamin $\mathrm{E}$ in those at risk may be decreased because of its importance as chain reaction terminator in the lipid peroxidation process. Interestingly, erythrocyte GSH levels were not only associated with increased pneumoconiotic risk, but also dramatically reduced in subjects with chronic bronchitis symptoms reported at follow-up. Remarkably, all "enzymatic" antioxidants (GST, Gpx, SOD and catalase) were increased in those with rapid decline in FEV 1 versus those with a slow decline. Since longitudinal decline was analysed retrospectively, our observations could be explained as a compensatory upregulation of enzymatic antioxidants in those with reduced "nonenzymatic" antioxidant status. Reduced intake of Vitamin A [Morabia et al. 1989], as well as Vitamin C and E [Morabia et al. 1989, Schwartz and Weiss 1994, Britton et al. 1995] is associated with increased risk of progressive lung function loss and airway obstruction. Others have shown reduced GSH levels in bronchoalveolar lavage fluid in subjecst with obstructive lung disease [Linden et al. 1995].

Finally, this study enabled us to test the actual involvement of oxidative stress in coal dust induced respiratory disorders. We found that in CWP reduced erythrocyte GSH and Vitamin $\mathrm{E}$ content were risk factors while increased activities of catalase and $\mathrm{SOD}$ in red cells tended to be risk factors. At the same time, an over-all increase in enzymatic antioxidant capacity identified subjects, with a rapid retrospective lung function decline. GST activity showed a contrasting discriminative power; those with lower GST activity were at increased risk for progression of coal workers pneumoconiosis; however a lower lung function decline was observed in this group. Underscored by recent findings (GST polymorphism) we believe that this finding deserves further investigation. Although pneumoconiotic and nonpneumoconiotic endpoints were 
independent, it can not be ruled out that both effects may be closely related to each other. On the one hand, a reduced intake of antioxidants -associated to lung function loss- may also be a risk factor for coal dust induced pulmonary fibrosis. On the other hand, coal dust exposure and its associated generation of ROS could also play a role in some of the nonpneumoconiotic effects such as chronic bronchitis or chronic lung obstruction. As such, measurement of multiple antioxidants in the peripheral blood may be relevant for surveillance and screening of (retired) coal workers. To our opinion, these observations should be extrapolated to other cohorts of coal (or other mineral) dust exposed subjects, to validate whether antioxidant status patterns of the peripheral blood as reported above can be considered as useful biological markers in subjects chronically exposed to coal (i.e. mineral) dust.

Acknowledgements This analysis could only occur due to the effort of many people contributing in this follow-up study. In particular, we thank all coal miners and the Kempense Steenkoolmijnen NV for their willingness to cooperate in this study, Luc Lenaerts (MD), Marc van Sprunde] (PhD) and Luc Marien (MD) for reading the chest radiographs. John Engelen and Chris Evelo for antioxidant determinations and Tim Jorna and Thim Derhaag for lung function measurenents. Bernard Préat is acknowledged for the calculation of cumulative dust exposure, and Fons Kessels (PhD) for his advice in the discriminant analysis. This study is supported by grant no. 7263/03/092 of the European Community for Steel and Coal. 

PART 2

Cytokines 



\title{
Chapter 8
}

\section{EPIDEMIOLOGICAL EVALUATION OF MONOCYTE TNF- $\alpha$ RELEASE AS AN EXPOSURE AND EFFECT MARKER IN PNEUMONOCONIOSIS. A FIVE YEAR FOLLOW UP STUDY AMONG COAL WORKERS.}

\author{
Roel PF Schins and Paul JA Borm. \\ Department of Health Risk Analysis and Toxicology, University of Limburg, \\ PO BOX 616, $6200 \mathrm{MD}$, Maastricht, the Netherlands.
}

Published in: Occupational and Environmental Medicine 1995;52:441-50.

\begin{abstract}
Objectives - To determine (a) reproducibility with previous cross-sectional findings, and $b$ ) the predictive value of initial release of tumour necrosis factor- $\alpha$ (TNF- $\alpha$ ) towards later progression of coalworkers' pneumoconiosis CWP.

Methods - Release of monocyte TNF- $\alpha$ after in vitro stimulation with coal mine dust, silica, and endotoxin was measured in 104 retired miners and was related to stage of CWP (chest radiograph) and cumulative exposure. A subgroup of 46 miners was screened by high resolution computed tomography (HRCT). Prospective analysis of TNF- $\alpha$ (40 out of 104 miners involved in the previous TNF- $\alpha$ study) was done by relating initial TNF- $\alpha$ to five year progression of CWP, measured by comparison of paired chest radiographs.

Results - As observed previously, dust stimulated release of TNF- $\alpha$ was increased in miners, especially in the early stages of pneumoconiosis. Cumulative exposure was related to pneumoconiotic stage but not to release of TNF- $\alpha$. This excluded TNF - $\alpha$ as a exposure marker. Initial concentrations (1987) of TNF- $\alpha$ were related to later progression of CWP. Miners who showed abnormally high dust stimulated release of TNF- $\alpha$ had an increased risk of progression in CWP (relative risk 8.1).

Conclusions - These results show (a) the significant involvement of TNF- $\alpha$ in pneumnconiosis in humans induced by coal dust and (b) that this routine test possibly constitutes a powerful tool to estimate individual prognosis of pneumoconiotic disease, even after the end of occupational exposure.
\end{abstract}




\section{Introduction}

Pulmonary fibrosis should be looked upon as a pathological process dependent upon many cell types, in which alveolar macrophages, lung eptihelium, fibroblasts, type II cells, PMN, and many other cells are involved [Elias et al. 1990; Crystal et al., 1991]. These cells are known to produce various factors (reactive oxygen species, cytokines, enzymes, etc.) that are thought to be crucial mediators of the hallmarks of pulmonary fibrosis: excessive fibroblast proliferation and collagen deposition [Crystal et al., 1991; Snyder, 1988]. Mediators that have been put forward, mostly from animal studies, are: Platelet derived growth factor- $\beta$ (PDGF- $\beta$ ), Tumor necrosis factor- $\alpha$ (TNF), Interleukin-1 $\beta$ and Interleukin-6 (IL-1 $\beta$ , $\mathrm{LL}-6)$ and prostaglandin-E2 (PGE2) in asbestosis [Brody 1993; Perkins et al., 1993] or TNF, IL- 1 and the Macrophage inflammatory proteins $1 \alpha$ and 2 (MIP- $1 \alpha$, MIP2) in silicosis [Piguet et al., 1990; Driscoll et al., 1990; Mohr et al., 1991; Driscoll et al., 1993]. The potential of many of these cell mediators as markers of exposure, disease susceptibility or effects has been raised many times, but studies on the relations between exposure, disease, and cytokine release in occupationally or environmentally exposed subjects are scarce.

In 1987 several case-control studies among coal miners were performed in our laboratory to look for a marker to explain disease among exposed miners with and without coal workers' pneumoconiosis (CWP). The serum concentration of procollagen type III peptide was measured to assess fibrotic activity versus pneumoconiotic stage [Janssen et al., 1992]. Another study focused on the relation between CWP and antioxidant expression [Engelen et al., 1990]. The in vitro secretion of TNF by peripheral blood monocytes [Borm et al., 1988] of healthy miners and miners with CWP, matched on age and underground working years, showed that the release of TNF in monocytes could be stimulated in vitro by coal mine dust, and that (healthy) coal miners had a higher release of monocyte TNF upon in vitro stimulation than did controls who were not expoesd to dust. Moreover, a decreasing trend in the release of TNF was seen along with the clinical progression of CWP possibly as a positive feedback mechanism to prevent concentration of fibrosis, and in healthy miners $(0 / 0)$ and miners with early stages of CWP $(0 / 1)$ a large variation in the release of TNF was found. We then suggested that people who showed values largely exceeding the normal range of controls who were not exposed to dust, were at risk of development of CWP and concluded that definitive proof for the role of mononuclear phagocyte-derived TNF in the pathogenesis of CWP and other fibrotic lung diseases has to originate from laboratory animal studies and prospective epidemiologic surveys among those at risk' [Borm et al., 1988]. Several years ago, Piguet et al. [1990] showed the crucial role of TNF fibrosis induced by silica with a mouse in vivo model. Since then other studies have firmly established the importance of TNF in lung fibrosis induced by silica 
[Drsicoll et al., 1990; Mohr et al., 1991; Phan et al., 1992]. Also in vitro studies have emphasised the involvement of TNF in the complex cytokine network of lung tissue [Elias et al., 1990; Perkins et al., 1990; Warren et Al., 1988; Everson and Chandler, 1992]. Lasalle and coworkers reported an increased spontaneous release of TNF by alveolar macrophages of active healthy coal miners and miners with CWP compared with controls [Lasalle et al., 1990]. The importance of these findings for the prevention of lung fibrosis induced by mineral dust remains an open question. In 1992 all miners involved in our cross sectional studies were recruited for a five year follow up to investigate whether our previous cross sectional findings on release of TNF in subjects exposed to dust and disease stage of CWP could be validated, and to determine the relative risk of development of CWP for coal miners who showed high in vitro release of TNF.

\section{Methods}

\section{STUDY GROUP}

Miners ( $n=156$, fig 1) from the Belgian coal mining industry "Kempense Steenkoolmijnen" involved in previous studies in 1987 [Borm et al., 1988; Engelen et al., 1990; Janssen et al., 1992] were asked to participate in the follow up study that included a chest radiograph, several lung function tests, medical and exposure history, and the blood variables TNF, PIIIP and red blood cell antioxidant enzymes (AOE). Chest radiographs taken in 1987 and in 1992 were scored according to International Labour Organisation classification rules [ILO, 1980] by an experienced panel of three physicians. A subgroup of 46 miners was screened by high resolution computed tomography (HRCT) by invited participation. Percentages of cases and control miners in the HRCT subgroup (i.e. $13 v 23$ ) were equal to those of the large cohort (i.e. $76 v 28$ ), and none of the variable; age, smoking habits or exposure in the subgroup were different from the large cohort $(n=104)$. A control group of 44 age matched men participated in the study on voluntary basis. Exclusion criteria for controls not exposed to dust were: working for more than one year in an occupational setting with chronic dust exposure (mining, cement works, ceramic industry, bakery, etc.) and one or more of: asthma, (chronic) bronchitis, and other chronic diseases of lung, liver and kidney. Based on these criteria, 29 controls were included in statistical analysis.

\section{TNF-assay}

Release of monocyte TNF was measured as described previously [Borm et al., 1988]. Briefly, blood $(3 \times 10 \mathrm{ml})$ was sampled and left overnight in tubes with EDTA at room temperature. White blood cells were isolated by buoyant density centrifugation (Lymphoprep-Nycomed), adjusted to concentrations of $0.5 \times 10^{7}$ 
cells $/ \mathrm{ml}$ and seeded $(0.5 \mathrm{ml})$ to tissue culture dishes (Costar TC-24) to allow adherence (repetitive incubation, agitation and washing). Adherent monocytes were stimulated with coalmine dust $(5 \mathrm{mg} / \mathrm{ml})$, silica particles $(0.5 \mathrm{mg} / \mathrm{ml})$ or with Escherichia coli 0111B4 LPS endotoxin (3 and $1000 \mathrm{ng} / \mathrm{ml}$ ). Silica and endotoxin were obtained from Sigma (St Louis USA); the coal mine dust (sampled from a coal pit) was obtained from the same batch as used previously [Borm et al., 1988]. Each person's baseline release of TNF was measured without addition of any in vitro stimulant. After 18 hours of incubation $\left(37^{\circ} \mathrm{C}, \mathrm{CO}_{2}\right)$ the cell free supernatants were stored at $-30^{\circ} \mathrm{C}$ until analysis by a specific sandwich ELISA for TNF as described previously [Borm et al., 1988; Debets et al., 1988].

\section{QUESTIONNAIRE}

An extensive questionnaire was sent to all participants in which medical complaints, medical history, smoking habits, and medication were asked for. Answers were all verified during personal interviews at the time of medical screening and by comparison with questionnaires from the data records of our preceding studies. Amount of smoking was expressed as: packets of cigarettes/week $x$ years (pack-years) smoked. One packet was considered to consist of 25 cigarettes; the smoking of one cigar was considered to be equivalent to 3 cigarettes; and $50 \mathrm{~g}$ (pipe-) tobacco to be equivalent to 75 cigarettes. Furthermore all subjects were subdivided into current, former, and never smokers.

Subjects were classified for medication and medical history based on the presence or absence of any medication during three weeks before blood sampling. Disease was determined in two ways; the presence or absence of any medical complaint for at least three days, and of specific disease of the lung, kidney, liver, or any other chronic disease for at least three years before blood sampling.

\section{EXPOSURE}

Much emphasis was put on a good estimation of exposure and several variables were used: (a) time worked underground, (b) time since first occupational exposure, (c) time since last occupational exposure, (d) cumulative exposure to respirable coal dust and quartz.

Years exposed, first exposure, and last exposure

Years exposed, time since first exposure and time elapsed since last occupational exposure were determined from the medical file and a personal interview at follow up. Exposure years were determined from the complete job history obtained from each subject and defined as the total number of years in which a subject worked underground in a coal mine. Long term absenteeism -for example, for disease or through special leave- as well as interim job changes were taken into account. Years since first exposure were defined as the interval between the year in which a miner started working underground and the time of 
our study. Time from last exposure was the interval between retirement and our follow up in months.

Calculation of cumulative dust exposure

Occupation history, gathered from the medical file and verified by a personal interview, was used to calculate exposure for all periods worked at and out of the coalfaces. The cumulative dust exposure was calculated by summation of the products of the yearly mean dust concentrations for the colliery where the miner was appointed with the average time worked underground during that year.

The formula was:

$$
F_{X}=\sum_{i=1}^{n}\left(C_{i} \cdot T_{X}\right) \quad,\left(g h m^{-3}\right)
$$

where, $\mathrm{E}_{\mathrm{X}}=$ cumulative dust exposure $(\mathrm{ghm}-3)$ of miner $\mathrm{x} ; \mathrm{C}_{\mathrm{i}}=$ mean dust concentration $\left(\mathrm{g} \cdot \mathrm{m}^{-3}\right)$ in year $\mathrm{i} ; \mathrm{T}_{\mathrm{X}}=$ time worked underground $(\mathrm{h})$ by miner $\mathrm{x} ; \mathrm{n}$ = years of employment.

The resulting individual exposure is expressed in $\mathrm{ghm}^{-3}$ of sampled air. The yearly mean dust concentrations $\left(C_{i}\right)$ were derived from dust measurements regularly made to check compliance with exposure limits in every coal face of the colliery. Since 1965 these measurements have been carried out with a STASER sampler (jointly developed by a Belgian manufacturer and the Dutch State Mines) at fixed points, $15 \mathrm{~m}$ from the face line, in the return end gate. The concentrations before 1965 have been estimated on the basis of preliminary studies carried out in preparation of the implementation of dust regulations. The total dust concentrations have been converted into "respirable" dust concentrations by the formula:

$$
\mathrm{rd}=1.1 \sqrt{\mathrm{td}} \quad,\left(\mathrm{ghm}^{-3}\right)
$$

where, $\mathrm{rd}=$ respirable dust concentration $(\mathrm{gm}-3)$; $\mathrm{td}=$ total $\mathrm{dust}$ concentration (gm-3), as measured.

This empirical relation (equation 2) was derived from experimental side-by-side instrument comparisons conducted in 1984-5 with the French CPM3 instrument and the STASER sampler under various mining conditions in the Belgian coal mines (144 paired measurements). The time worked underground was estimated for each subject assuming that on average 1000 hours a year were worked underground : six hours at work a shift (allowance being made for travelling 
time) $\times 220$ shifts a year $\times(100-25) / 100$ (where $25 \%$ is taken as a rate of absenteeism). The exposure outside the coal face was estimated on the basis of professional judgement of the "dustiness" of the occupation and the knowledge of normal respirable dust levels in that job (non-mechanised and mechanised headings, stone drivages, etc). The cumulative quartz exposure (mghm-3) has been estimated, based on the average quartz content of Belgian coalface $(5 \%)$ and stone drivage dust $(10 \%)$.

\section{RADIOGRAPHY}

\section{Protocol and classification}

Chest radiographs $(46 \times 46 \mathrm{~cm})$ were taken posterior to anterior at maximal inspiration (1.5 $\mathrm{m}$ distance, $70 \mathrm{kV}$ ) and scored according to the ILO criteria [ILO, 1980]. Scoring was done in plenary sessions where consensus had to be reached based on individual scores. The reading panel consisted of three occupational physicians of whom two were seeing more than 3000 chest radiographs a year, and one is a trained reader but had not been active as such for the last three years. Two readers in our follow up study had been involved with the original study. Standard ILO chest radiographs were used as reference to classification. Based on the $\Pi$ LO scores the cohort was subdivided into five groups: ILO classification $0 / 0$ (group 0 ), class $0 / 1$ (group 1 ), classes $1 / 0,1 / 1$ and $1 / 2$ (group 2 ), classes $2 / 1,2 / 2$ and $2 / 3$ (group 3 ) classes $3 / 2$ and $3 / 3$ (group 4), and finally, and $3 /+$ and subjects with progressive massive fibrosis (group 5). Furthermore miners were grouped as either "healthy coal workers" with ILO classification of $0 / 0$ (group 0 only) or as miners with evidence for CWP with ILO classification of $\geq 0 / 1$ (groups 1 to 5). Five year changes in pneumoconiosis were determined by comparison of each man's chest radiographs of 1987 and 1992. All radiographs were scored in a randomised blind reading session, as well as in pairs; the paired reading session was used as definitive evidence of progression as this direct comparison method allows the best validation of TNF as marker of five year changes in CWP.

\section{High Resolution Computed Tomography}

The HRCT scans were made (Somaton plus, Siemens, Erlangen, Germany) and visually scored by two radiologists as described elsewhere [Lamers et al., 1994] in a subgroup of 46 miners. Briefly, four HRCT slices, two in the upper lobes ( 3 and 5 $\mathrm{cm}$ above the carina) and two in the lower lobes ( 3 and $5 \mathrm{~cm}$ below the carina) were obtained at full inspiration in a supine position, and scored independently by two thoracic radiologists without knowledge of clinical findings. Each slice was visually assessed for the presence of non-calcified parenchymal opacities with almost the same basic principles as the ILO system for grading pulmonary disease on chest radiographs. The profusion score was converted to a linear scale that ranged from zero to 10 as follows: " $0 / 0=0,0 / 1=1,1 / 0=2,1 / 1=3,1 / 2=4$, $2 / 1=5,2 / 2=6,2 / 3=7,3 / 2=8,3 / 3=9$ and $3 /+=10^{\prime \prime}$ [Lamers et al., 1994; Rémi- 
Jardin et al., 1990]. The score of the eight lung fields was summed up and a total score was calculated. The mean score of both observers (correlation 0.86, $p<0.001$ ) was calculated for the purpose of this study. Our attention was mainly focused on the relation between TNF and HRCT lesions in a group of 35 miners without the classical diagnostic evidence for CWP (ILO $\leq 0 / 1)$.

\section{DATA EVALUATION AND STATISTICAL ANALYSIS}

Figure 8.1 schematically shows the composition of the study group. Exploratory analysis was performed on the 1992 cross-sectional data, to study the confounding effects of age, smoking, medication, and variables of exposure. Reproducibility with cross sectional data of 1987 was then evaluated by unpaired statistical analysis of data obtained in 1987 [Borm et al., 1988] $v$ data obtained in 1992 and by cross sectional analysis of 1992 data as previously described for the 1987 cross sectional data [Borm et al., 1988]. Longitudinal study analysis was performed by paired statistical analysis of 1987 and 1992 data and predictive values of variables of 1987 (prospective analysis) or 1992 (retrospective analysis) were related to five year changes in radiological severity of CWP.

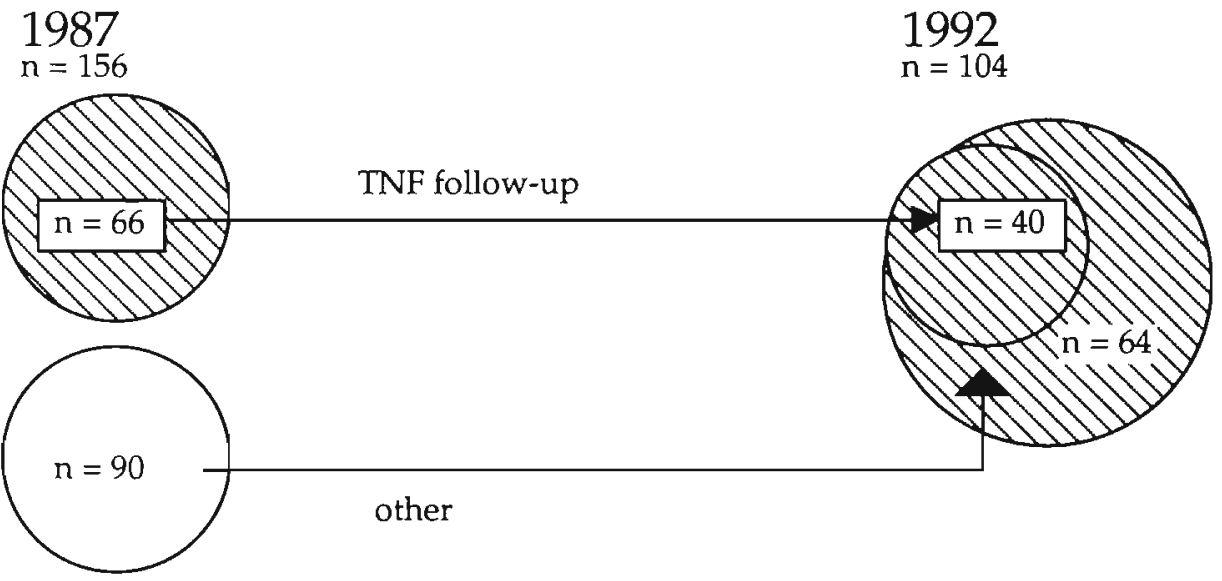

Figure 8.1. Construction of the follow up study among retired coal miners previously (1987) involved in cross-sectional studies on the role of TNF- $\alpha(n=66)$ or antioxidants $(n=90)$ in coal workers pneumoconiosis. The shaded area represents the groups in which TNF- $\alpha$ was determined. Data interpretation is done either by comparison of the TNF- $\alpha$ follow up group ( $n=40)$ (longitudinal analysis) or of the total 1992 cohort $(n=104)$ with the TNF- $\alpha$ group in $1987(n=66)$ (cross sectional analysis). Chest radiographs were taken for all miners in $1987(n=156)$, and for all miners involved in 1992 ( $n=104$ ), allowing 104 paired chest radiograph comparisons (determination of five year progression of CWP). From the 1992 cohort $(n=104), 46$ miners were screened by high resolution computed tomography by invited participation. 
Correlations between release of TNF and exposure, age, smoking were evaluated by multiple linear regression. Differences between miners without or with CWP and non-exposed controls, and effects of medication, medical history, and smoking were tested by the Kruskall Wallis test. Trend analysis between pneumoconiotic stage (chest radiographs, HRCT) and TNF, exposure, or other variables was analysed by Spearman's rank correlation. For this purpose, profusion scores were converted to a scale from 0 to 11 for chest radiograph classification and 0 to 80 for HRCT as already indicated. Positive predictive value of five year changes in chest radiograph, sensitivity, and specificity of initial release of TNF were determined. The relative risk of progression of CWP based on 1987 TNF data was determined with a cut off point of 2 SDs above the mean release of TNF measured in the non-exposed control group of 1987 [Borm et al., 1988]; Similarly, the odds ratio of cumulative dust exposure with progression of CWP was determined retrospectively, with the mean cumulative exposure of the 1992 cohort as a cut off point. Also, to determine the significance of our findings at various cut off points, receiver operating characteristics (ROC) curves were calculated [Hanley and McNeil., 1982; van der Schouw et al., 1993]. Statistical evaluations were made with STATGRAPHICS/6.0 (Manugistics, Rockville MD).

\section{Results}

\section{ANALYSIS OF EXPLORATORY DATA}

Age, smoking, medication and exposure

Table 8.1 shows group characteristics of the total study cohort of 1992. The average age of the control group not exposed to dust was significantly higher than the control miners. No correlation was observed between age and release of TNF in any of the groups (Table 8.2). Smoking habits did not differ among the three groups (Table 8.1), but release of monocyte TNF upon stimulation with coal dust was significantly inversely related to packyears in the miners ( $n=104$, Table 8.2). The dust stimulated release of TNF was significantly lower in ever smokers ( $1.68 \mathrm{ng} / \mathrm{ml}, \mathrm{n}=61)$ v never smokers (2.90 $\mathrm{ng} / \mathrm{ml}, \mathrm{n}=15)$ (ANOVA, $\mathrm{p}<0.01)$ but not significantly different between active smokers $(n=27)$ and nonsmokers $(n=49)$. Furthermore, smoking was related to release of TNF upon stimulation with coal dust and low concentration of endotoxin $(3 \mathrm{ng} / \mathrm{ml})$ in miners without CWP, and to high concentration of endotoxin $(1000 \mathrm{ng} / \mathrm{ml})$ in the controls not exposed to dust. In the controls not exposed to dust the average release of TNF upon stimulation with $1000 \mathrm{ng} / \mathrm{ml}$ endotoxin was significantly different for active smokers $(1.63 \mathrm{ng} / \mathrm{ml}, \mathrm{n}=16)$ v non-smokers $(3.16 \mathrm{ng} / \mathrm{ml}$, $\mathrm{n}=13$ ), and for (ever) smokers ( $1.85 \mathrm{ng} / \mathrm{ml}, \mathrm{n}=22) v$ never smokers $(3.76 \mathrm{ng} / \mathrm{ml}$, $\mathrm{n}=7$ ) (ANOVA, $\mathrm{p}<0.05$ ). Differences in release of TNF with respect to medication were not observed in any group. Miners with CWP had significantly higher 
cumulative exposure and underground working years than the control miners (Table 8.1). No significant relation was found between any of the exposure variables and release of TNF (Table 8.2).

Table 8.1 Group characteristics of the study cohort in $1992(n=133)$.

\begin{tabular}{lccc} 
& $\begin{array}{c}\text { Controls not } \\
\text { exposed to dust }\end{array}$ & $\begin{array}{c}\text { Controls healthy } \\
\text { miners }\end{array}$ & $\begin{array}{c}\text { Miners } \\
\text { with CWP }\end{array}$ \\
\hline Age (years) & $(\mathrm{n}=29)$ & $(\mathrm{n}=76)$ & $(\mathrm{n}=28)$ \\
Smoking (packyears) & $50.3(1.0)^{*}$ & $47.7(0.6)$ & $49.9(1.1)$ \\
Smoking (yes/no) & $115(20)$ & $111(12)$ & $150(24)$ \\
Smoking (ever/never) & $16 / 13$ & $28 / 48$ & $14 / 14+$ \\
Medication (yes/no) & $22 / 7$ & $62 / 14$ & $2.3 / 5$ \\
Cumulative exposure: & $8 / 21$ & $20 / 56$ & $7 / 21$ \\
$\quad$ & & & $130.9(9.3)^{* *}$ \\
$\quad$ dust (gh/m3) & - & $91.1(6.4)$ & $6751(461)^{* *}$ \\
Years underground & - & $4798(306)$ & $25.0(0.9)^{* *}$ \\
Years since first exposure & - & $22.2(0.4)$ & $30.7(1.0)$ \\
Months retired & - & $28.9(0.6)$ & $53.9(2.1)$ \\
\hline
\end{tabular}

The asterisks denote a significant difference towards the control miners group: * $p<0.05$ *tr $\mathrm{p}<0.01$ (Mann-Whitney); Values are mean (SEM); ${ }^{\dagger}$ Proportion of smokers/former smokers/never smokers in the ILO profusion categories was: $4 / 2 / 1(0 / 1) ; 1 / 2 / 2(1 / 0,1 / 1,1 / 2) ; 3 / 2 / 2(2 / 1,2 / 2,2 / 3)$; $4 / 2 / 0(3 / 2,3 / 3)$; and $2 / 1 / 0(3 /+$, PMF $)$

\section{REPRODUCIBILITY OF CROSS-SECTIONAL DATA}

\section{Reproducibility of TNF data}

Figure 8.2 shows the monocyte release of TNF for both cross-sectional studies in 1987 and 1992, for the controls not exposed to dust, the control miners, and miners with pneumoconiosis. Unpaired $t$ tests of all miners involved in the original study and in the entire follow up group showed that both the spontaneous release of TNF and the release of TNF upon stimulation with low endotoxin $(3 \mathrm{ng} / \mathrm{ml})$ in $1992(\mathrm{n}=104)$ were both significantly lower than in 1987 $(n=66)$; conversely, release of TNF stimulated with coal mine dust was significantly higher in 1992 than in the 1987 study ( $\mathrm{p}<0.05$ ). Spontaneous release of TNF and release of TNF upon stimulation with low endotoxin were significantly reduced in the healthy coal miners of $1992(n=76) v 1987(n=39)$ as well as in the miners with CWP of $1992(n=28) v 1987(n=27)$. On the other hand, stimulation with coal mine dust released significantly higher concentrations of TNF in the healthy miners of $1992 v 1987$, but not in the miners with CWP of 
$1992 v$ 1987. Release of TNF upon dust stimulation was also significantly increased in the controls not exposed to dust in $1992(\mathrm{n}=29) v$ the control group of 1987 ( $n=12)$. Other (unpaired) differences were not observed.

Table 8.2 Linear correlations between TNF and exposure, age, and smoking

Release of TNF by stimulation with:

\begin{tabular}{|c|c|c|c|c|c|c|}
\hline $\begin{array}{l}\text { ILO } \\
\text { classification }\end{array}$ & $\mathbf{n}$ & Spontaneous & $\begin{array}{c}\text { Endotoxin } \\
3 \mathrm{ng} / \mathrm{ml}\end{array}$ & $\begin{array}{l}\text { Endotoxin } \\
1000 \mathrm{ng} / \mathrm{ml}\end{array}$ & $\begin{array}{l}\text { Coal dust } \\
5 \mathrm{mg} / \mathrm{ml}\end{array}$ & $\begin{array}{c}\text { Silica } \\
0.5 \mathrm{mg} / \mathrm{ml}\end{array}$ \\
\hline \multicolumn{7}{|c|}{ cumulative dust $\left(\mathrm{ghm}^{-3}\right)$} \\
\hline $0 / 0$ & $(76)$ & 0.01 & 0.17 & 0.02 & 0.00 & 0.10 \\
\hline$\geq 0 / 1$ & $(28)$ & -0.28 & -0.10 & -0.02 & -0.20 & -0.06 \\
\hline Total & $(104)$ & -0.06 & 0.08 & 0.01 & -0.04 & 0.03 \\
\hline \multicolumn{7}{|c|}{ cum. quartz (mghm-3) } \\
\hline $0 / 0$ & $(76)$ & 0.03 & 0.19 & 0.02 & 0.05 & 0.11 \\
\hline$\geq 0 / 1$ & $(28)$ & -0.33 & -0.05 & 0.06 & -0.23 & -0.10 \\
\hline Total & (104) & -0.06 & 0.11 & 0.03 & -0.02 & 0.03 \\
\hline \multicolumn{7}{|c|}{ years underground } \\
\hline $0 / 0$ & (76) & -0.05 & 0.12 & -0.03 & -0.02 & 0.00 \\
\hline$\geq 0 / 1$ & (28) & 0.05 & 0.16 & 0.30 & 0.10 & 0.16 \\
\hline Total & $(104)$ & -0.04 & 0.12 & 0.08 & 0.02 & 0.05 \\
\hline \multicolumn{7}{|c|}{ years since first exposure } \\
\hline $0 / 0$ & $(76)$ & -0.22 & 0.01 & -0.06 & -0.17 & -0.18 \\
\hline$\geq 0 / 1$ & $(28)$ & 0.08 & -0.01 & 0.16 & -0.02 & 0.00 \\
\hline Total & (104) & -0.15 & 0.00 & 0.01 & -0.12 & -0.10 \\
\hline \multicolumn{7}{|l|}{ age (years) } \\
\hline $0 / 0$ & $(76)$ & -0.02 & 0.11 & -0.05 & 0.00 & 0.07 \\
\hline$\geq 0 / 1$ & (28) & 0.05 & 0.14 & 0.09 & 0.16 & 0.29 \\
\hline Total & (104) & 0.05 & 0.07 & -0.03 & 0.03 & 0.05 \\
\hline Control + & $(29)$ & 0.13 & -0.09 & 0.07 & 0.09 & -0.20 \\
\hline \multicolumn{7}{|c|}{ snuking (packyenurs) } \\
\hline $0 / 0$ & $(76)$ & 0.16 & $-0.24^{*}$ & -0.12 & $-0.27^{*}$ & -0.14 \\
\hline$\geq 0 / 1$ & $(28)$ & -0.04 & 0.00 & 0.03 & -0.27 & -0.07 \\
\hline Total & (104) & 0.09 & -0.16 & -0.07 & $-0.26^{* *}$ & -0.11 \\
\hline Control $t$ & $(29)$ & -0.15 & -0.25 & $-0.46^{*}$ & -0.20 & -0.09 \\
\hline
\end{tabular}

$r=$ correlation cuefficient significant at ${ }^{*} p=0.05$ or ${ }^{*} p=0.01 ;{ }^{\dagger}$ Not exposed to dust 

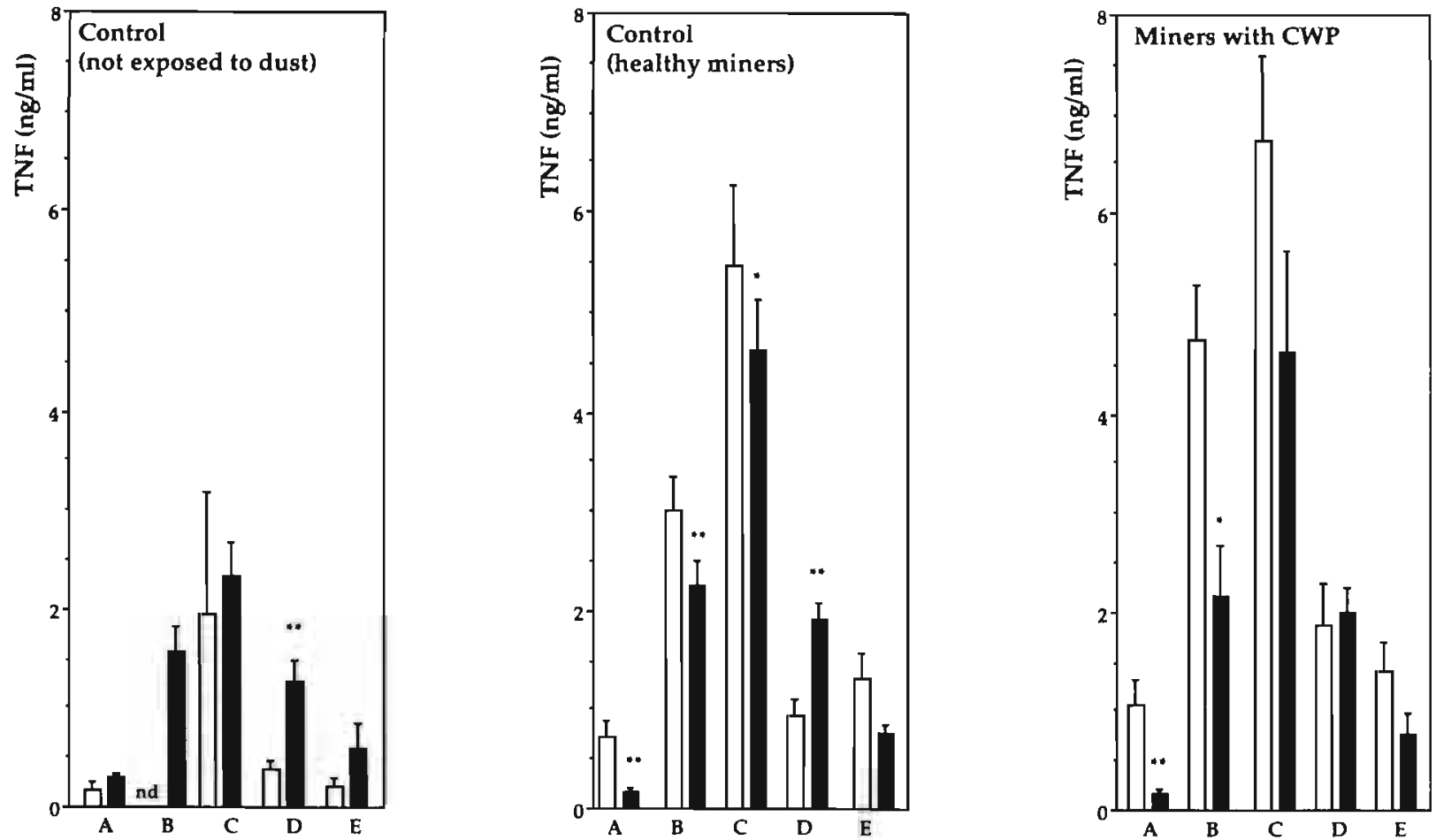

Figure 8.2. Mean release of TNF- $\alpha$ from blood monocytes in 1987 (open bars) and 1992 (solid bars). Data were obtained from 12 and 29 (1987 is 1992) control subjects not exposed to dust, from 39 and 76 control miners exposed to dust, and from 27 and 28 miners exposed to dust who had coal workers' pneumoconiosis. All values are means (SEM) and are expressed in $\mathrm{ng} / \mathrm{ml}$. The alphabetic symbols depict spontaneous release of TNF- $\alpha$ (A), or release of TNF- $\alpha$ in response to $3 \mathrm{ng} / \mathrm{ml} \mathrm{LPS} \mathrm{(B),} 1,000 \mathrm{ng} / \mathrm{ml} \mathrm{LPS} \mathrm{(C),} 5 \mathrm{mg} / \mathrm{ml}$ coal mine dust (D), or $0.5 \mathrm{mg} / \mathrm{ml}$ silica (E). Testing for significance was with the Mann-Whitney $U$ test; the asterisks indicate iignificant differences between 1987 and 1992 data (* $p<0.05 ; * * 0<0.01)$. nd $=$ not determined. 
Despite these differences in absolute values, the 1992 cross sectional comparison of release of TNF between controls not exposed to dust, control miners and miners with CWP confirmed our previous observations (Fig. 8.2). Again, release of TNF in the controls not exposed to dust was significantly lower than in the control miners upon stimulation with high endotoxin $(1000 \mathrm{ng} / \mathrm{ml})$ and stimulation with silica. At present also release of TNF in the controls not exposed to dust was significantly lower than the control miners and miners with CWP upon stimulation with coal mine dust (Mann-Whitney U-test $\mathrm{p}<0.05$ ). Contrary to previous findings, however, none of the stimuli resulted in significant differences in release of TNF between miners with or without CWP; moreover the spontaneous release of TNF was significantly higher in the controls not exposed to dust $v$ both miners' groups (Mann-Whitney, $\mathrm{p}<0.01$ ).

\section{TNF and profusion score}

Figure 8.3 shows the individual TNF data of the total follow up group $(n=104)$ upon stimulation with coal mine dust as a function of profusion score. As previously [Borm et al., 1988], a large variation in response of TNF upon stimulation with coral mine dust was observed in the healthy miners group.

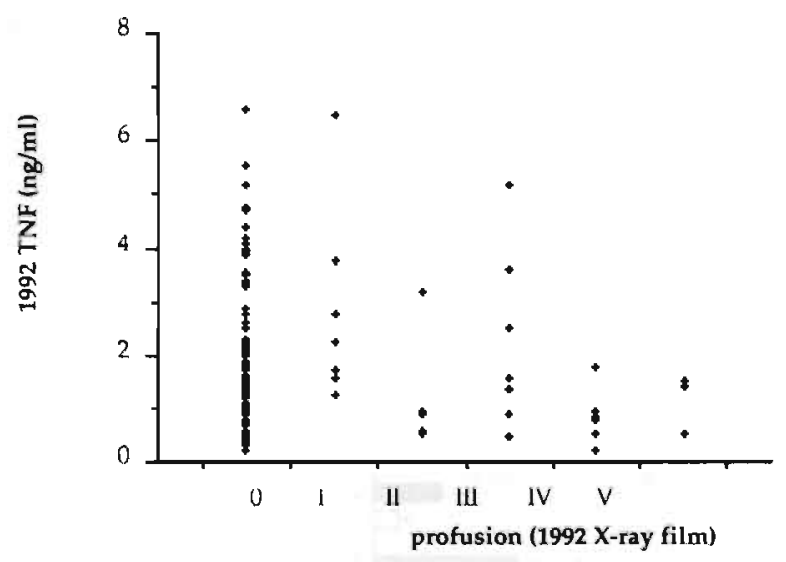

Figure 8.3. Release of TNF- $\alpha$ in 1992 in $\mathrm{ng} / \mathrm{ml}$ of peripheral blood monocytes of individual miners stimulated with coal dust as a function of 1992 chest radiograph classification.

Profusion categories are $0(0 / 0)$, I $(0 / 1)$, II $(1 / 0,1 / 1,1 / 2)$, II $(2 / 1$, $2 / 2,2 / 3)$, IV $(3 / 2,3 / 3), \mathrm{V}(3 /+$, progressive massive fibrosis).

In miners in the early stages of CWP, release of TNF was significantly increased and a decrease in release of TNF was found along with the increasing stage of CWP that seems to continue in PMF. Spearman rank correlation tests showed a significant downward trend in release of TNF with increasing profusion score starting from the ILO score $0 / 1$, upon stimulation with coal dust ( $n=28, \rho=-0.41$, $p<0.05)$, silica $(n=28, \rho=-0.39, p<0.05)$ and to a lesser degree with low endotoxin ( 3 
$\mathrm{ng} / \mathrm{ml}, \mathrm{n}=28, \mathrm{p}=-0.32, \mathrm{p}<0.1)$. In the HRCT subgroup $(\mathrm{n}=46)$ a downward trend of release of TNF was also found with increasing visual score of lesions in miners starting from ILO class $0 / 1$; however this trend did not reach significance for any stimulus $(n=13, p>0.1)$. In miners without the classic ILO evidence for CWP the release of TNF was not related to HRCT score, except for release of TNF stimulated with high endotoxin $(1000 \mathrm{ng} / \mathrm{ml})$. Interestingly, this increased with increasing number of lesions (Spearman, $\rho=0.35, p<0.05, n=33$ ).

Table 8.3 Group characteristics of the miners involved in the TNF study of $1987(n=66)$ and the subgroup involved in the follow up $(n=40)$.

\begin{tabular}{|c|c|c|}
\hline & $1987(n=66)$ & $1992(n=40)$ \\
\hline Age (years) ${ }^{*}$ & $49.4(0.76)$ & $48.1(1.04)$ \\
\hline Smoking (cigarettes/day) & $6.1(1.00)$ & $4.8(1.15)$ \\
\hline Smokers (yes/no) & $32 / 34$ & $17 / 23$ \\
\hline Medication (yes/no) ${ }^{*}$ & $10 / 56$ & $6 / 34$ \\
\hline Years underground (in 1987) & $21.2(0.49)$ & $20.7(0.70)$ \\
\hline Years underground (in 1992) ${ }^{\dagger}$ & $22.3(0.41)$ & $21.9(0.57)$ \\
\hline Time retired (months) ${ }^{\dagger}$ & $45.9(2.00)$ & $43.7(2.75)$ \\
\hline \multicolumn{3}{|l|}{ I.L.O. classifications $\neq$} \\
\hline $0 / 0$ & 39 & 25 \\
\hline $0 / 1$ & 7 & 4 \\
\hline $1 / 0,1 / 1,1 / 2$ & $9(=2,5,2)$ & $5(=1,2,2)$ \\
\hline $2 / 1,2 / 2,2 / 3$ & $8(=0,6,2)$ & $4(=0,4,0)$ \\
\hline $3 / 2,3 / 3$ & $3(=0,3)$ & $2(=0,2)$ \\
\hline \multicolumn{3}{|l|}{ Release of TNF (ng/ml)§ } \\
\hline Spontaneous & $0.86(0.14)$ & $0.93(0.21)$ \\
\hline Endotoxin $(3 \mathrm{ng} / \mathrm{ml})$ & $3.72(0.31)$ & $3.65(0.34)$ \\
\hline Endotoxin $(1000 \mathrm{ng} / \mathrm{ml})$ & $6.00(0.59)$ & $6.06(0.77)$ \\
\hline Coal mine dust & $1.32(0.20)$ & $1.17(0.16)$ \\
\hline Silica & $1.35(0.20)$ & $1.12(0.19)$ \\
\hline
\end{tabular}

Values are mean (SEM); ${ }^{*}$ data from the 1987 study; ${ }^{\dagger}$ data from personal records in th mine (1992);

Fchest radiograph in 1987; $\S_{\text {see text for details }}$ 


\section{LONGITUDINAL DATA ANALYSIS: RESULTS OF FIVE YEAR FOLLOW}

Follow up characteristics

Ultimately 104 coal miners (67\% of the original cohort) participated in our follow up (Fig. 8.1) and 40 out of $66 \mathrm{coal}$ miners involved in the TNF study [Borm et al., 1988] attended our follow up measurements. No selection bias was found for age, smoking, or time underground as was found in the original study (Table 8.3). Both groups did not differ for medication (yes/no, $\chi$ 2-test), or for pneumoconiotic stage (1987 chest radiograph, $\chi 2$-test), or for mean release of TNF (Table 8.3). During the follow up interval all miners had left active mining and there had been no occupational exposure for at least four years in half of our miners and at least for two years in more than $85 \%$ of our miners. No selective loss to follow up from active mining had been taken place (Table 8.3). The mean (SD) age of retirement in our total follow up group $(n=104)$ was 44.7 (5.1). Despite retirement a significant proportion of the cohort $(n=104)$ showed progression of CWP. Based on the paired readings of the chest radiographs, it was shown that during this five year follow up period four out of 80 healthy coal miners contracted CWP $(5 \%)$, and that in 11 cases existing CWP progressed $(46 \%)$. In two miners also progressive massive fibrosis (PMF) was established $(3 / 2 \mathrm{rA}$ and $2 / 3 \mathrm{rA})$. From the miners of the original TNF study who were involved in the follow up study ( $n=40)$, one out of 30 control miners developed new CWP, and five out of 10 miners had progression of already existing CWP.

\section{Longitudinal analysis of TNF in the follow up group}

In the TNF follow up group, paired tests of the individual data of 1987 and 1992 $(n=40)$, showed significant differences in spontaneous release of TNF, release of TNF upon stimulation with low endotoxin and with coal dust $(\mathrm{p}<0.05)$. Significant correlations between the TNF data of 1987 and 1992 were found upon stimulation with coal mine dust $(r=0.44, \mathrm{p}<0.01)$ or low dose endotoxin $(r=0.35$, $\mathrm{p}<0.05)$. These correlations were also significant in the miners without CWP $(n=28, p<0.01, r=0.49)$, and in all subjects (control miners and miners with CWP) without progression during the five year follow up ( $n=33, p<0.05, r=0.43$ ) for stimulated release of TNF with coal dust as well as with $3 \mathrm{ng} / \mathrm{ml}$ endotoxin (data not shown). Upon stimulation with $1000 \mathrm{ng} / \mathrm{ml}$ endotoxin no significant correlation was observed in the whole TNF follow up group, but interestingly, a significant relation was found only in the six miners with five year progression of CWP $(n=6, p<0.05, r=0.89)$. Correlations in the spontaneous release of TNF, or the release upon stimulation with silica were not found in any (sub)group.

\section{$T N F$, exposure and progression}

Multiple linear regression demonstrated that TNF is not a marker of exposure as release of TNF in either 1992 or 1987 was not related to individual cumulative (dust or quartz) exposure or to any of the other exposure variables in the TNF 
follow up subgroup $(n=40)$. Retrospective analysis showed, however, that the cumulative exposure was significantly related to progression of CWP. The odds ratio of cumulative exposure $v$ progression of CWP was 3.72 (significant at a $90 \%$ C.I), with the mean cumulative exposure $(100 \mathrm{gh} / \mathrm{m} 3)$ as a cut off for high and low exposure. In the TNF follow up subgroup $(n=40)$, cumulative exposure was also significantly related to progression at various cut off levels. Figure 8.4 shows both these observations and that all but one miner whi showed progression (or development) of pneumoconiosis between 1987 and 1992 originally had a release of TNF that exceeded the normal range in 1987. The relative risk of progression in CWP for miners who showed abnormal in vitro release of TNF was 8.1 (1.2$55.0,90 \%$ C.I.), with the cut off for dust stimulated release of TNF of 2 SDs above the mean of the controls not exposed to dust.

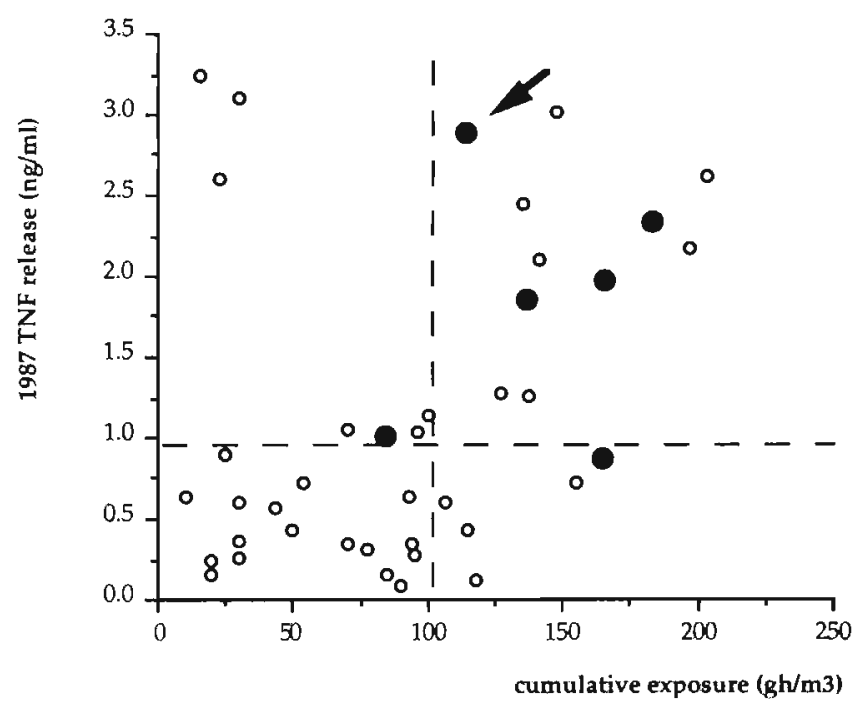

Figure 8.4. Graphical illustration of the relative risk for miners with high (> mean $+2 \times \mathrm{SD}$ ) release of TNF- $\alpha$ (1987) and high ( $>$ mean) cumulative exposure to dust in the progression of CWP compared with all miners involved in the TNF- $\alpha$ follow up study $(n=40)$. Note that no correlation is present between INF- $\alpha$ and exposure $(r 2=0.069)$ and that the solid circles, which indicate the individuals with progression ( $n=6$ ), are in the right quadrants (high TNF- $\alpha$ and high exposure) of the scheme. Thearrow indicates the subject with first diagnosed CWP. In the upper right quadrant, age, smoking habits, cumulative exposure and 1987 or 1992 TNF- $\alpha$ release were not different between the miners with progression of CWP $(n=4)$ and those without progression $(n=7)$. 
To determine sensitivity and specificity at any cut off point of TNF upon the different in vitro stimuli, ROC curves were calculated [van der Schouw et al., 1993]. Figure 8.5 shows ROC curves for spontaneous release of TNF and for release of TNF upon stimulation with $3 \mathrm{ng} / \mathrm{ml}$ endotoxin and coal dust. The strongest association between high TNF and progression was found release of TNF stimulated by dust, with an area under the curve (AUC) of $74 \%$. No clear associations were observed in all other options $(44 \%<$ A.U.C. $<55 \%)$.

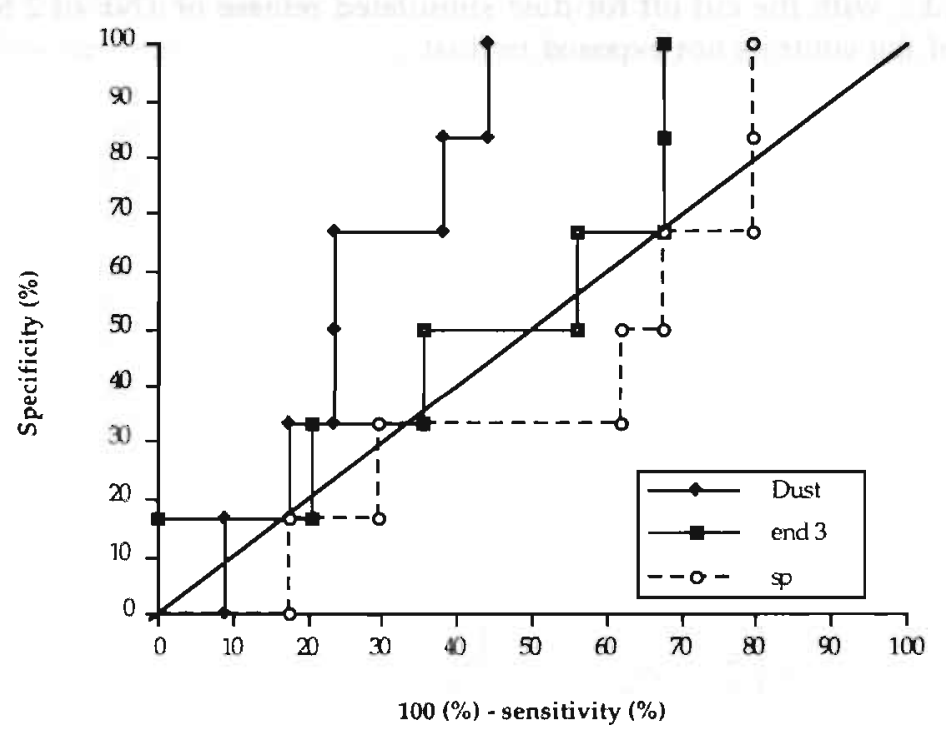

Figure 8.5. Receiver operator characteristic (ROC) curves of monocyte releae of TNF- $\alpha$ in 1987 as indicator for the progression of CWP. The diagonal with an area under the curve (AUC) of $50 \%$, marks the theoretical relation of an indicator without discriminative power. A perfect ROC: association would be an inverse L-shaped curve in the upper left comer of the figure (AUC $=100 \%$ ). The area under the curve was $44 \%$ for spontaneous release of TNF (sp), $55 \%$ for the release of TNF upon stimulation with $3 \mathrm{ng} / \mathrm{ml}$ endotoxin (end 3), and $74 \%$ for release of TNF upon stimulation with coal dust (Dust). 


\section{Discussion}

The findings of our follow up study among retired coal workers lend support for an essential role of TNF in the genesis of pulmonary fibrosis induced by mineral dust in general[ Elias et al., 1990; Perkins e al., 1993; Piguet et al., 1990; Driscull ct al., 1990; Mohr et al., 1991; Warren et al., 1988; Everson and Chandler 1992] and CWP in particular [Borm et al., 1988; Lasalle et al., 1990; Cosset et al., 1991; Vanhée et al., 1993]. Moreover this study shows that, when carefully applied, release of monocyte TNF is a valid tool for the prognosis of CWP. The large variation of release of TNF found in healthy miners $(0 / 0)$ and miners at early stage of CWP $(0 / 1)$ proved to be a scientific basis for a susceptibility marker: those with a high release of TNF upon stimulation with coal dust are at higher risk of development or progression of CWP. Although to test this hypothesis only analysis of the relation of the original release of TNF concentrations with prospective changes in CWP was needed, TNF was also measured in present study, and several other aspects were included. This also allowed us to test the possible feedback mechanisms in the progression of CWP, as already suggested, the reproducibility of the assay and cross sectional results, and effects of the end of occupational exposure (retirement). Individual exposure assessment and HRCT screening, both mainly focused on miners with normal chest radiographs, were included to investigate (or exclude) the role of in iitro stimulated release of TNF as a marker of exposure or as a marker of early (fibrotic) effects.

Cross sectional findings of the present study were similar to those of the original study [Borm et al., 1988]. Although significant differences in absolute TNF levels were found, release of TNF upon stimulation with coal dust and the low concentration of endotoxin in 1987 and 1992 were significantly correlated. This relation was present in the entire follow up group, as well as in all subjects without progression of CWP. Interestingly, TNF concentrations were not correlated in miners with progression of CWP, except for TNF released upon stimulation with the high concentration of endotoxin $(n=6)$. This suggests feedback on release of TNF during active fibrosis. In contrast with our previous observations, no significant differences in TNF were found between miners with and miners without CWP, and the spontaneous release of TNF was significantly higher in the non-exposed controls. Both changes are likely due to the retirement of the miners in our present study-that is a reduced in vivo stimulation or priming. This is supported by findings of Lasalle et al [1990] in active and retired miners. Threse authors found that baseline release of TNF in alveolar macrophages was significantly higher in active miners. We therefore suggest that scrum concentrations of TNF and baseline (spontaneous) release from monocytes or alveolar macrophages reflect an acute response to inhaled dusts, whereas the in vitro release by peripheral blood monocytes shows the chronic adaptation of a subject. In our study such an adaptation may be due both 
to long term (and subsequent end of) exposure to coal dust and the effect of early pathogenic processes. Evidence for the involvement of exposure is the large variation of release of monocyte TNF in miners $v$ controls. The role of early disease also seems evident, considering that the highest release of TNF was found in miners in the early stages of CWP.

Although release of TNF was highest in the early stages of CWP according to chest radiographs, HRCT analysis showed that release of TNF by men within this category was not related to the number of lesions found by HRCT. Therefore the variation in in vitro release of TNF, the basis of evaluation of this biomarker, is unlikely to be biased by early fibrotic processes. Also all evidence that could show that TNF is an exposure marker is negative; no relation between release of TNF and any exposure index (cumulative exposure, exposure years, time since first exposure) could be shown. This does not mean, however, that TNF is not affected by exposure, as TNF concentrations in coal miners are clearly different from those in subjects without chronic exposure to mineral dust. Perkins et al. reported similar findings in subjects with long term exposure to asbestos without evidence of asbestosis [Perkins et al., 1993]. The lack of relations between release of TNF and exposure indices coul be considered as a false negative result because of the original selection of subjects in the first case-control design [Borm et al., 1988], matched on age and years underground, or to errors in the estimation of individual exposure. One could suggest that differences in exposure are therefore too small to detect any significant correlation with other variables. This is, however, contradicted by the large variation of cumulative exposure (four fold) at the same period of years underground, and the biological plausibility of the existing relations between exposure and CWP: calculated cumulative dust exposure correlated significantly with pneumoconiotic stage $(r=0.34, p<0.001$, chest radiographs; $r=0.44, p<0.005, H R C T)$, and was significantly higher in miners with progression of CWP.

Interestingly, the incidence of CWP and progression to more severe stages in this small cohort was significant, especially when taking into account that all miners retired early during the follow up. As progression was derived from paired readings of chest radiographs, no doubt is exists on the identity of progressed cases. The extent of progression is subject to variation due to differences in size of some radiographs, increased weight of most subjects, and interpretation error. Our findings are in line with epidemiologic data showing that progression is highest in advanced stages of CWP [Attfield and Wagner, 1992] and that incidence of CWP is more frequent among men who have left industry than among those in the active work force [Soutar et al., 1986; Mahieu, 1990]. Therefore, there are extensive populations at risk of CWP throughout the world and methods of screening and surveillance by careful monitoring of the health of these miners and ex-miners are needed. In our study, both cumulative dust exposure and release of TNF in 1987 were significantly related to five year 
changes in CWP. The (retrospective) estimate of the odds ratio for cumulative exposure was 3.72, and the relative risk for progression of CWP for someone with an abnormal (> mean $+2 \mathrm{SD}$ ) release of TNF was 8.1 in this cohort. However, there is a subtle difference: dust exposure determines the prevalence of CWP after several years, whereas release of TNF determines the individual risk at a certain exposure (Fig. 8.4).

It should be emphasized that in vitro release of TNF induced by coal mine dust was the best predictor of progression, whereas baseline release of TNF was not related to five year progression of CWP (Fig. 8.4). Again this raises the question of priming of the monocytes by external exposure to mineral dust. Apart from our original suggestion of this phenomenon in coal miners [Borm et al., 1988], priming of macrophages was reported by Mohr et al. in rats exposed to silica [Mohr et al., 1991], coal miners [Lasalle et al., 1990] and asbestos workers [Perkins et al., 1993]. Local lung modulatory factors have been suggested in the upregulation of TNF secretion by monocytes [Pantelidis et al., 1993] but also autocrine pathways may be involved in the priming of macrophages [Tanner et al., 1992; Pfeffer et al., 1993]. We think that priming of monocytes in coal miners reflects the response to chronic release of factors from the lung loaded with coal dust. Studies with alveolar macrophages, and lung tissue of coal miners [Lasalle et al., 1990;Vanhée et al., 1993] confirmed this hypothesis and also suggested that as well as silica other compounds in coal dust and smoking [Lasalle et al., 1990; Gosset et al., 1991; Dubar et al., 1993] are important in the release of such factor(s). For example, in our study stimulated release of TNF was lower in miners who smoked, whereas the baseline response tended to be higher in non-smokers. Several results suggested the presence of feedback mechanisms that counteract the processes (dust exposure, smoking and inflammatory response) that upregulate monocyte release of TNF. Previously, and at follow up, we noted a downward trend of stimulated release of TNF as CWP stage increased. Moreover, in 1992 release of TNF was not such a good predictor of progression of CWP as it was in 1987. Also correlations between assays in 1987 and 1992 were only significant in miners without progression of CWP and not miner whose disease had progressed. We could not test the possible effect of feedback on TNF as a marker for progression of CWP in our cohort, because only retired miners were involved. Therefore, we are not able to discriminate between the effects of stopping exposure and failure of feedback as determinants of monocyte release of TNF. This remains an interesting subject for future investigations in a larger cohort of active miners.

In conclusion, our data add in vivo human evidence to the already existing in vitro and animal data that TNF is a crucial mediator in lung disease induced by mineral dust. It is appreciated that the size, original selection, and follow up interval of our cohort could limit the significance of our findings. The original 
study had a case-control design matched for age and exposure to investigate the involvement of TNF in CWP. To reach acceptable response and statistical power (cases with progression), the cohort had to be supplemented with other miners, screened by us for different purposes in the same year. Nevertheless, the reproducibility of cross-sectional findings was excellent. Moreover, we showed that TNF data are not biased by either exposure or stage of disease and showed a high relative risk of high release of TNF at equal exposures and radiological outcomes. Its specificity as a marker is illustrated by the fact that none of the subjects with a normal release of TNF showed progression of disease. Determination of the ROCs (Fig. 8.5) showed that the significance of our findings was not solely due to the specific choice of the cut off in the present study, and allows the applicability of this marker in other cohorts of (retired) coal miners. Future follow up will increase the number of cases as the average age of our cohort is well below the current average to contract CWP [Soutar et al., 1986; Mahieu, 1990] and can help to get a more reliable estimate of the relative risk. Other estimates can come from studies that will use this method as a tool for health surveillance in, for example, miners who leave the active work force.

Acknowledgements. We are indebted to all coal miners and the Kempense Steenkoolmijnen NV for their cooperation in this study. We thank Luc Lenderts (MD), Luc Mariën (MD) and Marc van sprundel (MD), PhD) for reading the chest radiographs, Rob Lamers (MD) for HRCT analysis, Bernard Préat for cumulative exposure estimations, and Thim Derhaag for technical assistance. Finally we acknuwledge Wim Buurman (PhD) for providing the TNF assay and useful advice. This study is supported by grant no. 7203/103/092 of the European Community for Steel and Coal. 


\title{
Chapter 9
}

\section{PLASMA LEVELS OF THE SOLUBLE TUMOUR NECROSIS FACTOR (TNF) RECEPTORS ARE INCREASED IN COAL MINERS.}

\author{
Roel PF Schins and Paul JA Borm. \\ Department of Health Risk Analysis and Toxicology, University of Limburg, \\ PO BOX 616, 6200 MD Maastricht, the Netherlands.
}

Published in: European Respiratory Journal 1995;8:1685-63.

\begin{abstract}
Among other cytokines, Tumor Necrosis Factor-a (TNF) is considered to play a key role in the development of mineral dust related fibrosis. Previously, we showed that ex-vivo release of TNF by peripheral blood monocytes is a marker for progression of coal workers' pneumoconiosis (CWP). Since soluble TNF receptors (sTNF-Rs) are believed to play an important regulatory role in systemic effects of TNF, we measured plasma levels of sTNF-R55 and sTNF-R75 in coal miners with $(n=28)$ or without $(n=76)$ CWP and in non-exposed controls $(\mathrm{n}=29)$. sTNF-R75 levels were significantly increased in miners with CWP $(2.09 \pm 0.44 \mathrm{ng} / \mathrm{ml})$ versus the non-exposed controls $(1.86 \pm 0.23 \mathrm{ng} / \mathrm{ml})$. Neither sTNF-R55 nor sTNF-R75 were related to exposure, stage of pneumoconiosis, smoking, or (spontaneous or $e x$-vivo induced) monocyte TNF-release. sTNF-R55 was increased in subjects with medication (especially those using cardiovascular drugs); upon exclusion of these subjects, also sTNF=R55 was found also to be significantly increased in CWP. In conclusion, bearing in mind a confounding effect of medication, soluble TNF receptors are elevated in plasma of retired miners with CWP. These observations further support the important role of TNF-mediated pathways in the pathogenesis of mineral dust related fibrosis.
\end{abstract}

\section{Introduction}

Occupational exposure to mineral dusts can lead to pulmonary fibrosis Crystal et al., 1991]. The pathogenesis of lung fibrosis involves highly complicated processes of intercellular communication by peptides ieleased from and to various immune cells and lung target cells in a phenomenon appropriately referred to as "cytokine network" [Elias et al., 1990]. Previously, we and others showed that silica as well as coal mine dust, can stimulate the release of monocyte-/macrophage derived proinflammatory cytokines, such as Tumor Necrosis Factor- $\alpha$ (TNF) [Borm et al., 1988; Driscoll et al., 1990; Gosset et al., 1991], Interleukin-1 $\beta$ (几-1) [Driscoll et al., 1990; Schmidt et al., 1984; Lassale et al., 1990], Interleukin-6 (IL-6) [Gosset et al., 1991] and the Macrophage Inflammatory 
Interleukin-6 (IL-6) [Gosset et al., 1991] and the Macrophage Inflammatory Proteins $1 \alpha$ and 2 [Driscoll et al., 1992]. With regard to dust exposure in man we found that monocyte TNF-release upon ex-vivo stimulation with coal dust and silica (and endotoxin) was increased in (retired) miners with or without radiological evidence for simple coal workers pneumoconiosis (CWP) compared to subjects never exposed to mineral dust [Borm et al., 1988; chins and Borm, 1995]. In line with our observations in monocytes of coal workers, higher TNF secretion was also observed in macrophages of patients with progressive massive fibrosis (PMF) compared to simple pneumoconiosis patients and control subjects [Lassale et al., 1990]. More recently, elevated mRNA levels of TNF (and IL-6) have been observed in lungs of pneumoconiosis patients [Vanhée et al., 1993]. Crucial evidence for the importance of TNF in silica-induced lung fibrosis was demonstrated by Piguet $e t$ al., who showed a downregulatory effect on fibrosis by TNF-antibody treatment [Piguet et al., 1990].

Two distinct receptors of TNF, i.e. a $55 \mathrm{kDa}$ type (TNF-R55) and a $75 \mathrm{kDa}$ type (TNF-R75) have been identified, and both receptors are expressed on many different cell types and tissues [Brockhaus et al., 1990; Loetscher et al., 1990; Schall et al., 1990; Smith et al., 1990]. Shedding of the extracellular parts of these TNF receptors from white blood cells and probably also other cell types leads to two soluble TNF receptor types, known as sTNF-R55 and sTNF-R75 [Jäätelä et al., 1990; Beuteler et al., 1993]. Since naturally occurring soluble TNF receptor levels are believed to play an important regulatory role in systemic effects of TNF [Jäätelä et al., 1990; Sheperd, 1991] plasma TNF receptor levels may represent a feedback mechanism to the proinflammatory systemic action of TNF, or an (in)direct reflection of TNF-related pathological mechanisms within the lung. In the present study, we determined soluble TNF receptors in plasma of coal miners with or without CWP and in controls never occupationally exposed to dust. The purpose of the study was to evaluate the relation of soluble TNF receptors with cumulative (silica-) dust exposure and the biological effects of pneumoconiosis disease [Borm, 1994]. Secondly, it was designed to evaluate the relation between soluble TNF receptor levels and monocyte TNF release [Schins and Borm, 1995; Borm, 1994] in non-exposed subjects and in coal workers either in the presence or absence of lung fibrosis.

\section{Materials and methods}

As a part of a prospective cohort study among coal workers [Schins et al., 1994], soluble receptors level were determined in the plasma of coal miners $(n=104)$ and non dust-exposed controls $(n=29)$, after written informed consent. Prior to the blood sampling, a questionnaire, including informed consent was obtained from each participant. The study was conducted according to the Helsinki 
declaration of 1975. On the day of blood sampling, a chest radiograph was made of each miner. Confirmation of questionnaires and data from medical files and job history were obtained by personal interviews. Subjects were classified as smokers, non-smokers, former smokers or life time nonsmokers; the amount smoked was expressed in pack years (packs/week $x$ years smoked). Smoking habits were verified using a personal interview and smoking data from previous cross-sectional studies [Borm et al., 1990; Janssen et al., 1992] to minimize reporting bias. The same was done for medical history: All subjects were divided into two categories, i.e. those who used any kind of medication and those who did not use medication at least three days (or in the case of antibiotics: one week) prior to blood sampling.

Severity of pneumoconiosis was obtained by classification of chest radiographs by three occupational physicians [Schins et al., 1994], according to the standard protocol of the International Labour Organization [ILO, 1980]. High resolution computed tomography (HRCT) was performed in a subgroup of 46 miners on a voluntary basis as described previously [Lamers et al., 1994]. The cumulative dust exposure was determined from job-exposure matrices as described previously [Jorna et al., 1994]. Blood (10 ml EDTA-tube) was sampled, transferred to the lab at $4^{\circ} \mathrm{C}$, and subsequently centrifuged. Plasma was stored at $70^{\circ} \mathrm{C}$. Soluble receptor levels of R-55 and R-75 were determined by sandwich ELISA as described by Leeuwenberg [Leeuwenberg et al., 1994]. Briefly, immunoassay plates (Nunc Maxisorp, Roskilde, Denmark) were coated overnight at $4^{\circ} \mathrm{C}$ with monoclonal anti-TNF-R55 or anti-TNF-R75. Plates were saturated with $1 \%$ BSA in PBS $(\mathrm{w} / \mathrm{v})$ for $1 \mathrm{~h}$ at room temperature. Test samples were added to the plates and incubated for $2 \mathrm{~h}$ at room temperature. Plates were washed and subsequently incubated with biotin-labeled rabbit anti-sTNF-R antiserum for $1 \mathrm{~h}$, followed by washing of the plates and addition of peroxidase labeled streptavidin. Peroxidase activity was determined spectrophotometrically after addition of 3,3',5,5'-tetramethylbenzidine substrate at $450 \mathrm{~nm}$ by ELISAreader. Present ELISA methods show no interference between sTNF-R and TNF, as previously reported [Leeuwenberg et al., 1994. In the same subjects, monocyte TNF release was determined as reported previously [Schins and Borm, 1995; Borm, 1994].

\section{Statistical analysis.}

Study group characteristics were evaluated by the Student's t-test. Relations between the soluble TNF receptors R-55 and R-75 and age, pack years, and exposure (i.e. miners only) were evaluated by linear regression. Multiple comparisons were evaluated by stepwise regression analysis. All statistical evaluations were made using STATGRAPHICS version 6 (Manugistics Inc., Rockville Maryland 20852. USA). 


\section{Results}

In the plasma of all individuals, levels of both soluble receptors were readily detectable (i.e. $>200 \mathrm{pg} / \mathrm{ml}$ ). Mean and standard deviations were $1.44 \pm 0.44 \mathrm{ng} / \mathrm{ml}$ (sTNF-R55) and $1.97 \pm 0.46 \mathrm{ng} / \mathrm{ml}$ (sTNF-R75). Plasma levels of sTNF-R75, but not sTNF-R55, were significantly elevated in miners with pneumoconiosis compared to subjects never exposed to dust (Table 9.1). Age in the non-exposed subjects was not different from miners with CWP, but was higher than in miners without radiological signs of pneumoconiosis (Table 9.1). Cumulative exposure was higher in miners with CWP compared to the reference miners. Plasma levels of sTNF-R55 and sTNF-R75 were not different between current, former or never smokers in any of the subgroups, and not related to the severity of pneumoconiosis as defined by conventional chest radiograph or by high resolution computed tomography (data not shown).

With the exception of age no linear correlations were present between the soluble TNF receptors and other variables as smoking, exposure, and monocyte TNF release (Table 9.2). A stepwise multiple regression analysis on both soluble TNF receptors using age, cumulative exposure (miners only), pack years smoked, medication, spontaneous and coal dust-stimulated monocyte TNF release as independent variables was performed in each study group. In the reference miners both age $(t=2.39, p<0.025)$ and medication $(t=-2.32, p<0.025)$ gave a significant fit in the model to sTNF-R55, but in the non-exposed controls only medication $(t=-3.12, p<0.005)$ was significantly related to sTNF-R55. Subjects using medication had increased plasma levels of sTNF-R55 compared to subjects without medication (Table 9.3). Among the reference miners (but not in other groups), age was significantly higher in those having medication (Table 9.3), thus explaining the correlation between age and plasma sTNF-R55 levels in this subgroup. None of the variables was related to sTNF-R75 levels in any of the subgroups. Moreover, no relation was found between plasma levels of sTNF-R55 or STNF-R75 and (spontaneous or induced) monocyte TNF release (Table 9.2).

Because of the significant effects of medication on sTNF-R55 levels, statistical analysis was repeated after exclusion of all subjects using medication. The difference in sTNF-R75 between CWP miners and non-exposed controls remained significant (Fig. 9.1, panel B), but now also an increase $(p<0.06)$ of sTNF-R55 was observed in miners with CWP. This confounding effect of medication on sTNF-R55 and the increased tendency in STNF-R55 in pneumoconiosis is illustrated in Fig. 9.1 (panel A). Again in this analysis no significant correlations were present between the soluble receptors and smoking or exposure, nor was a correlation observed with age. The previously significant correlation with age in the reference miners $(n=76)$, was not present in the references without medication $(n=55, p>0.25)$. Again, no relation between soluble 
receptors and monocyte TNF release was seen in subjects not using medication (data not shown).

Table 9.1 Study group characteristics and plasma levels of the soluble TNF receptors STNF-R55 and sTNF-R75 in miners with coal workers pneumoconiosis, reference miners and a non dust-exposed control group.

$\begin{array}{ccc}\text { Controls } & \text { Coal miners } & \text { Coal miners } \\ \text { Nonexposed } & \text { References } & \text { with CWP }\end{array}$

\begin{tabular}{|c|c|c|c|c|c|}
\hline & & & $(n=29)$ & $(n=76)$ & $(n=28)$ \\
\hline \multirow{2}{*}{\multicolumn{3}{|c|}{ Age yrs }} & $50.3 \pm 5.2 *$ & $47.7 \pm 5.0$ & $49.9 \pm 5.6$ \\
\hline & & & $42-59$ & $37-60$ & $41-60$ \\
\hline \multirow{2}{*}{\multicolumn{3}{|c|}{ Smoking pack-yrs }} & $102.8 \pm 100.5$ & $110.8 \pm 106.5$ & $150.4 \pm 128.5$ \\
\hline & & & $0-465$ & $0-520$ & $0-476$ \\
\hline \multirow[t]{7}{*}{ Medication } & - no & $\mathrm{n}$ & 21 & 56 & 21 \\
\hline & - yest (I) & $\mathrm{n}$ & 0 & 5 & 2 \\
\hline & (II) & n & 2 & 4 & 0 \\
\hline & (III) & $\mathrm{n}$ & 1 & 3 & 1 \\
\hline & (IV) & $\mathrm{n}$ & 0 & 4 & 2 \\
\hline & (V) & $\mathrm{n}$ & 4 & 5 & 1 \\
\hline & (VI) & $\mathrm{n}$ & 1 & 2 & 1 \\
\hline \multirow{2}{*}{\multicolumn{2}{|c|}{ Cumulative dust exposure }} & $\mathrm{ghm}^{-3}$ & - & $91.1 \pm 56.4$ & $130.9 \pm 49.5^{* *}$ \\
\hline & & & & $10-280$ & $23-203$ \\
\hline \multirow[t]{2}{*}{ sTNF-R55 } & $\mathrm{ng} / \mathrm{ml}$ & & $1.47 \pm 0.48$ & $1.39 \pm 0.43$ & $1.52 \pm 0.44$ \\
\hline & & & $0.78-2.55$ & $0.36-2.67$ & $0.96-3.08$ \\
\hline \multirow[t]{2}{*}{ sTNF-R75 } & $\mathrm{ng} / \mathrm{ml}$ & & $1.86 \pm 0.23$ & $1.96 \pm 0.53$ & $2.09 \pm 0.44 \#$ \\
\hline & & & $1.47-2.51$ & $1.05-3.68$ & $1.44-3.35$ \\
\hline
\end{tabular}

All values (i.e. meantstandard deviation and range) are evaluated by the Student's t-test. *p<0.05 and **p. (1.01 significantly different from reference miners.; \#p<0.01 significantly different from non dust-exposed controls; CWP = miners with coal workers 'pneumoconiosis (ILO chest radiograph $>0 / 0)$; + Subjects were classified according to six treatment categories: (I) Inhalation drugs, i.e. $\beta$-agonists, corticosteroids, and theophylline ( $n=7)$; (II) cardiovascular drugs, i.e. bblockers and diuretics $(\mathrm{n}=6)$; (III) Immunosuppressive and anti-inflammatory drugs and allopurinol ( $n=5$ ); (IV) antibiotics ( $\mathrm{n}=6$ ); (V) $\mathrm{H}_{2}$-agonists, laxantia, anti-psychotica and thyroid function drugs $(n=10)$; (VI) others $(n=4)$. In reference miners three subjects used two types of medication. 
Table 9.2 Correlation of plasma levels of the soluble TNF receptors sTNF-R55 and sTNF-R75 with age, smoking, exposure, and monocyte TNF release.

\begin{tabular}{|c|c|c|c|c|}
\hline & $\begin{array}{l}\text { Soluble } \\
\text { receptor }\end{array}$ & $\begin{array}{c}\text { Controls } \\
\text { Nonexposed }\end{array}$ & $\begin{array}{l}\text { Coal miners } \\
\text { References }\end{array}$ & $\begin{array}{l}\text { Coal miners } \\
\text { with CWP }\end{array}$ \\
\hline & & $(n=29)$ & $(n=76)$ & $(n=28)$ \\
\hline \multirow[t]{2}{*}{ Age yrs } & sTNF-R55 & $0.02(0.92)$ & $0.34(0.03)$ & $0.20(0.30)$ \\
\hline & sTNF-R75 & $0.12(0.54)$ & $0.19(0.10)$ & $-0.18(0.37)$ \\
\hline \multirow[t]{2}{*}{ Smoking pack-yrs } & sTNF-R55 & $0.19(0.32)$ & $0.08(0.51)$ & $-0.09(0.64)$ \\
\hline & sTNF-R75 & $0.10(0.60)$ & $0.00(0.99)$ & $-0.04(0.83)$ \\
\hline \multirow[t]{2}{*}{ Underground working yrs } & sTNF-R55 & - & $0.19(0.11)$ & $-0.16(0.43)$ \\
\hline & sTNF-R75 & - & $0.16(0.18)$ & $-0.06(0.77)$ \\
\hline \multirow[t]{2}{*}{ Cumulative exposure $\mathrm{ghm}^{-3}$} & sTNF-R55 & - & $0.09(0.45)$ & $0.10(0.60)$ \\
\hline & sTNF-R75 & - & $0.04(0.70)$ & $0.06(0.75)$ \\
\hline \multicolumn{5}{|l|}{ TNF release $\mathrm{ng} / \mathrm{mL}$} \\
\hline \multirow[t]{2}{*}{ Spontaneous } & sTNF-R55 & $-0.09(0.66)$ & $0.13(0.26)$ & $-0.06(0.76)$ \\
\hline & sTNF-R75 & $-0.05(0.79)$ & $0.11(0.35)$ & $-0.08(0.70)$ \\
\hline \multirow[t]{2}{*}{ L.PS, $3 \mathrm{ng} / \mathrm{mL}$} & sTNF-R55 & $0.18(0.35)$ & $0.17(0.14)$ & $-0.03(0.90)$ \\
\hline & sTNF-R75 & $-0.11(0.59)$ & $0.15(0.19)$ & $-0.11(0.57)$ \\
\hline \multirow[t]{2}{*}{ L.PS, $1000 \mathrm{ng} / \mathrm{mL}$} & sTNF-R55 & $0.04(0.84)$ & $0.06(0.60)$ & $-0.05(0.80)$ \\
\hline & sTNF-R75 & $-0.17(0.39)$ & $0.09(0.42)$ & $-0.02(0.90)$ \\
\hline \multirow[t]{2}{*}{ Coal dust, $5 \mathrm{mg} / \mathrm{mL}$} & sTNF-R55 & $0.33(0.08)$ & $0.06(0.63)$ & $0.18(0.36)$ \\
\hline & sTNF-R75 & $0.13(0.51)$ & $0.07(0.52)$ & $0.01(0.93)$ \\
\hline \multirow[t]{2}{*}{ Silica, $0.5 \mathrm{mg} / \mathrm{mL}$} & sTNF-R55 & $0.35(.06)$ & $0.12(0.29)$ & $0.26(0.18)$ \\
\hline & sTNF-R75 & $0.09(.66)$ & $0.00(0.96)$ & $0.11(0.58)$ \\
\hline
\end{tabular}

All values are linear correlation coefficients ( $\mathrm{p}$-values); $\mathrm{CWP}=$ miners with coal workers pneumoconiosis (ILO chest radiograph $>0 / 0$ ); LPS $=$ Lipopolysaccharide endotoxin. 
Table 9.3 Effect of medication on plasma levels of soluble TNF receptors, and its relation with age, smoking and cumulative dust exposure

\begin{tabular}{|c|c|c|c|c|c|c|c|}
\hline & \multirow[b]{2}{*}{ Unit } & \multicolumn{2}{|c|}{ non-exposed controls } & \multicolumn{2}{|c|}{ reference miners } & \multicolumn{2}{|c|}{ miners with CWP } \\
\hline & & medication & no medication & medication & no medication & medication & no medication \\
\hline & & $(n=8)$ & $(n=21)$ & $(n=20)$ & $(n=56)$ & $(n=7)$ & $(n=21)$ \\
\hline age & (years) & $49.9 \pm 5.9$ & $50.4 \pm 5.4$ & $50.1 \pm 5.4^{*}$ & $46.9 \pm 4.7$ & $48.7 \pm 6.1$ & $50.3 \pm 5.5$ \\
\hline smoking & (pack years) & $139 \pm 90$ & $106 \pm 111$ & $99 \pm 87$ & $115 \pm 143$ & $178 \pm 109$ & $141 \pm 136$ \\
\hline cumulative exposure & $\left(\mathrm{ghm}^{-3}\right)$ & - & - & $101 \pm 67$ & $88 \pm 52$ & $132 \pm 43$ & $131 \pm 53$ \\
\hline sTNF-R55 & (ng/ml) & $1.86 \pm 0.65 * *$ & $1.32 \pm 0.29$ & $1.63 \pm 0.50^{* *}$ & $1.30 \pm 0.38$ & $1.57 \pm 0.35$ & $1.50 \pm 0.47$ \\
\hline sTNF-R75 & (ng/ml) & $1.88 \pm 0.18$ & $1.85 \pm 0.25$ & $2.10 \pm 0.54$ & $1.91 \pm 0.51$ & $2.15 \pm 0.62$ & $2.07 \pm 0.38$ \\
\hline
\end{tabular}




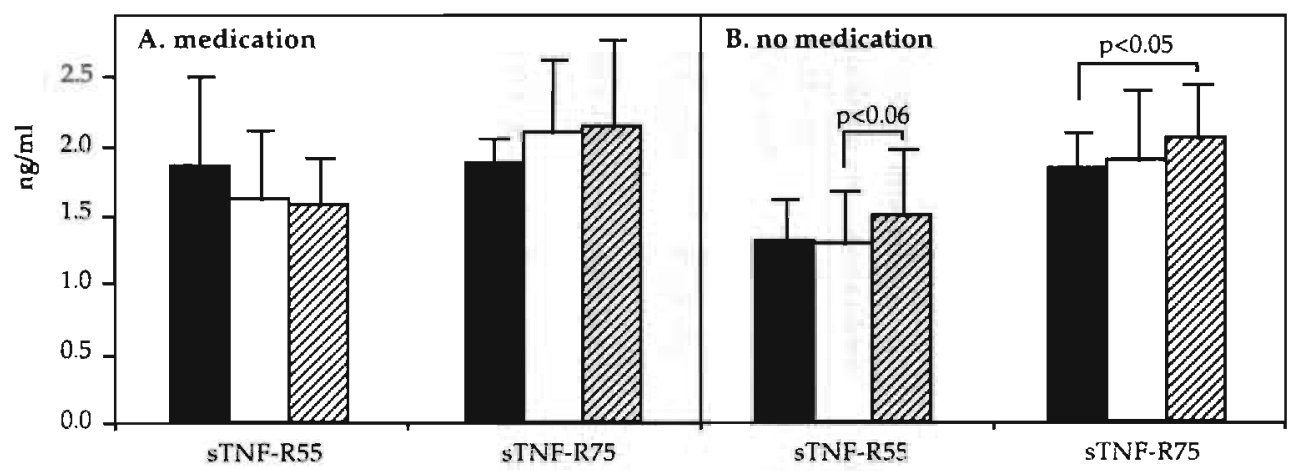

Figure 9.1. Effect of medication on plasma levels of the soluble Tumor Necrosis Factor Receptors (sTNF-R55 and sTNF-R75) in non-exposed controls (solid bars), reference miners (open bars), and miners with coal workers pneumoconiosis (dashed bars). Panel A represents receptor levels of subjects with medication ( $\mathrm{n}=35$ ); panel B shows all subjects without medication. Bars represent mean and standard deviations, evaluated by the Student's t-test. See table 9.1 for details on group sizes.

\section{Discussion}

To the best of our knowledge, this study is the first to describe a significant increase in plasma soluble TNF receptors in (simple) coal workers pneumoconiosis. sTNF-R75 levels were significantly increased in miners with CWP compared to the non-exposed controls. Controls (non-exposed), as well as reference miners, who used medication at the time of blood sampling also had increased levels of sTNF-R55, but upon statistical exclusion of these subjects, sTNF-R55 was also increased in CWP. No effect of smoking was seen and a relation of sTNF-R55 with age could be attributed to the effect of medication.

In the present study, no relation was found between monocyte TNF release and plasma levels of both soluble TNF receptors. Several reasons can explain for this lack of correlation. Firstly, it is unlikely that monocytes are the main source of STNF-R in vivo. Secondly, monocyte TNF release was determined ex-vivo in standardized culture conditions, where cells were seeded at equal concentrations in culture dishes, while plasma sTNF-R levels might be related to individual variations in white blood cell concentrations. Unfortunately, no differential or total cell counts of the blood were available. 
Since all miners in our study were retired, acute effects of coal dust exposure can be excluded. Moreover, plasma receptor levels were not related to cumulative dust exposure. In the miners with CWP $(n=28)$, a clear relation with disease severity, as determined by conventional chest radiograph or HRCT could not be established (data not shown). From these observations, one might suggest that levels of sTNF-R55 and sTNF-R75 could play a role in the pathogenesis of CWP.

Although many cytokines and growth factors are known to be involved in pulmonary fibrosis, the role of the "early" proinflammatory cytokine TNF- $\alpha$ as a central mediator in mineral dust related fibrosis is underscored by several in vitro and in vivo data [Borm et al., 1988; Driscoll et al., 1990; Gosset et al., 1991; Lassale et al., 1990; Vanheé et al., 1993; Mohr et al., 1991; Perkins et al., 1993]. The recent discovery of soluble cytokine antagonists -and particularly the soluble TNF receptors R55 and R75- have put things in a new perspective: soluble cytokine receptors could, on the one hand, act as powerful antagonists of various cytokine actions, but, on the other hand, increase cytokine related effects by a systemic carrier function of shortlived cytokines [Beutler et al., 1993; Ulich et al., 1993]. Piguet et al. demonstrated a downregulatory effect of TNF-antibodies [Piguet et al., 1990], and more recently of STNF-R55 [Piguet et al., 1994] in mineral dust related fibrosis. Doing so, they not only proved the crucial role of TNF in this disease, but they also elucidated a possible (therapeutic) role of soluble TNF receptors in mineral dust related fibrosis. Increased levels of soluble TNF receptors have been observed in several chronic and acute diseases[Aderka et al., 1991; Naylor et al., 1993; Latini et al., 1994; Shapiro et al., 1993; Tilg et al., 1993]. In chronic lung disease sTNF-R55 and R75 were found to be elevated in the bronchoalveolar lavage fluid of patients with idiopathic pulmonary fibrosis and sarcoidosis [Walker et al., 1994]. In line with our observations on monocyte TNF release in coal miners [Borm et al., 1988; Schins and Borm, 1995; Borm, 1994], sTNF-R55 and sTNF-R75 therefore also may play a role in the pathogenesis of coal workers' pneumoconiosis.

Unlike sTNF-R55, plasma levels of sTNF-R75 in the present study were clearly elevated in the plasma of miners with simple coal workers pneumoconiosis. Moreover, sTNF-R75 levels were not affected by medication. This might be in line with recent observations that TNF induced receptor shedding from peripheral blood neutrophils is specific to TNF-R75 [Porteu et al., 1994]. Similarly, the (slower) release of TNF receptors form mononuclear cells in response to LPS is a process also predominantly observed for TNF-R75 compared to TNF-R55 [Leeuwenberg et al., 1994].

From above data one might consider the applicability of plasma determination of soluble TNF receptors with regard to monitoring, as previously suggested for high ex-vivo monocyte TNF release as a positive risk factor for disease progression of CWP [Schins and Borm, 1995]. However, one should be aware of confounding factors on soluble TNF receptor measurements. Since the 
kidney is believed to be the main clearance route of soluble TNF receptors, any abnormality in renal function would result in altered plasma levels of both receptors [Bemelmans et al., 1993]. Shapiro et al. have also stressed the importance of appropriate blood processing for sTNF-R determinations [Shapiro et al., 1993]. In the present study, we observed an effect of medication on plasma levels of sTNF-R55. Due to a limited number of subjects using medication, we can only speculate on the action of specific types of medication although higher plasma levels of sTNF-R55 were found in those subjects using cardiovascular drugs compared to subjects without medication $(p<0.05, n=6)$. Whether (in)direct action of the drugs, or on the other hand the (history of) cardiovascular pathogenesis in these medicated subjects alters plasma sTNF-R55 kinetics (e.g. transcription, internalization, shedding, renal clearance) remains to be elucidated. However, it should be realized that in this small subgroup other effects may as well account for increased sTNF-R55 levels.

In conclusion, both sTNF-R55 and sTNF-R75 are increased in coal workers pneumoconiosis, although differences in receptor levels with exposed or nonexposed control groups may be confounded by the effect of medication used at time of blood sampling. The increased levels of soluble TNF receptors further support the important role of TNF in pneumoconiosis in coal workers. Interactions between TNF release and the antagonistic actions of soluble TNF receptors could be important in the development or progression of CWP. If so, measurement of these receptors may be of additional value in routine screening of dust exposed individuals. Support for this should be derived from a prospective study among coal workers, allowing a concomitant evaluation of TNF and its soluble receptors as risk markers.

Acknowledgements. The authors thank all coal miners and the Kempense Steenkoolmijnen NV for their willingness to cooperate in this study; Marc van Sprundel, Luc Lenaerts, and Luc Mariën for reading the $X$-rays; Bernard Préat for the calculation of individual cumulative exposure; Wim $A$. Buurman from the Department of Surgery, University of Limburg, The Netherlands, for useful advice and for providing antibodies and standards for STNF-R55 and R75 ELISA. This study was supported by grant no. 7263/03/092 of the European Community for Steel \& Coal. 


\title{
MULTIPLE CYTOKINES AS BIOMARKERS IN COAL DUST EXPOSURE AND PNEUMOCONIOSIS: TNF- $\alpha$, IL-6, AND TGF- $\beta$.
}

\author{
Roel PF Schins 1 , Philippe Gosset ${ }^{2}$, Benoit Wallaert ${ }^{2}$, Paul JA Borm ${ }^{1}$. \\ 1 Department of Health Risk Analysis and Toxicology, University of Limburg, \\ PO BOX 616, 6200 MD Maastricht, the Netherlands. \\ 2 INSERM U416, Institut Pasteur, 1 rue du Pr Calmette - BP 245, 59019 Lille CEDEX, France.
}

\begin{abstract}
Previously we showed that the individual risk for progression of Coal Workers Pneumoconiosis (CWP) is related to abnormal release of Tumor Necrosis Factor- $\alpha$ (TNF) from monocytes stimulated in vitro with coal dust. In this study we investigated Transforming Growth Factor- $\beta 1$ (TGF) and Interleukin-6 (IL-6) in the same cohort, since in line with TNF (1) they are released from coal dust stimulated alveolar macrophages, and (2) their significance is demonstrated in animal models of (silica) fibrosis as well as in lung of CWP patients. Spontaneous as well as coal- and silica dust-stimulated release of TGF and IL-6 was evaluated by ELISA in the same monocyte supernatants (18 hours) previously used to determine TNF release, including the supernatants from non-exposed individuals $(n=29)$, miners without CWP ( $n=76)$ and with CWP $(n=28)$. As previously reported, coal- and silicastimulated, but not spontaneously released TNF levels were higher in miners compared to non-exposed subjects. Spontaneous as well as coal dust- $(5 \mathrm{mg} / \mathrm{mL})$ and silica- $(0.5 \mathrm{mg} / \mathrm{mL})$ stimulated TGF release was significantly higher in miners compared to non-exposed individuals. However, TGF release from dust- or silica- stimulated monocytes was significantly lower than baseline release. IL-6 release was not different between the groups, and, unlike silica, stimulation with coal dust increased IL-6 release compared to spontaneous IL-6 release. Remarkably, dust- stimulated TGF and TNF release were positively correlated in the non-exposed individuals $(\rho=0.55, p<0.01$ ), but negatively correlated in the miners with CWP $(p=-0.55, p<0.01)$. Multiple logistic regression analysis showed that specific patterns of these cytokines released from monocytes are related to chronic coal dust exposure as well as resultant pneumoconiosis. In particular, the relative release of TGF and TNF from coal dust stimulated monocytes seems a powerful marker of progression of CWP. Follow-up will elucidate the validity of these cytokines as multiple risk markers in CWP.
\end{abstract}




\section{Introduction}

Pneumoconiosis caused by chronic inhalation of mineral dusts including asbestos, silica and coal dust are among the most widespread occupational diseases [Cullen et al., 1990]. Chronic inhalation of coal dust may lead to coal workers pneumoconiosis (CWP), characterized by a relatively mildly interstitial fibrosis at early stage disease [Gross, 1983; Attfield and Wagner, 1992]. Against the background of this simple CWP, occasionally progressive massive fibrosis (PMF) may develup, characterized by large lesions, severe fibrosis and collapse of lung parenchyma [Flint, 1988]. Regarding their potent stimulatory or inhibitory actions, several cytokines have been considered to amplify, respectively counteract the development or progression of fibrosis in man [Elias et al., 1990]. In mineral dust induced fibrosis, the alveolar macrophage is considered to play an important role [Heppleston and Styles, 1967; Takemura et al., 1989], and in vitro stimulation of these cells with mineral dust particles results in the release of many factors, including Tumor Necrosis Factor- $\alpha$ (TNF), Interleukin-1 (IL-1), Interleukin-6 (IL-6), Platelet-Derived Growth Factor (PDGF), Transforming Growth Factor- $\beta$ (TGF), Insuline-like Growth Factor-I (IGF-I) [Schmidt et al., 1984; DuBois et al., 1989; Lasalle et al., 1990; Perkins et al., 1993; Vanhée et al., 1994].

In vivo and in situ studies in animal models and fibrotic patients have identified some crucial cytokines with respect to mineral dust induced fibrosis. The role of TNF was demonstrated by Piguet and colleagues, in a mouse-model of fibrosis, using bleomycin as well as - more specifically with regard to pneumoconiosis- using silica [Piguet et al., 1989; 1990]. In both models, addition of TNF antibodies resulted in a significant attenuation of fibrogenesis, while concommittant addition of recombinant TNF further increased fibrogenic response. TNF as well as IL-6 was found in most of the cells from the nodular lesions [Piguet et al., 1990]. More recently, mRNA levels of TNF and Interleukin6 (IL-6) were found to be elevated in lungs of CWP and PMF patients [Vanhée et al., 1995]. Transforming Growth Factor- $\beta$ (TGF) has also be found in bleomycin models of fibrosis [Raghow et al., 1989; Piguet et al., 1989], however unlike TNF, and IL-6, no elevated TGF was seen in the silica model [Piguet et al., 1990]. Furthermore, alveolar macrophages (obtained by bronchoalveolar lavage) from CWP patients were found to release increased amounts of TNF, IL-6, PDGF, TGF, and IGF-1 in vitro, compared to alveolar macrophages from control subjects [Gosset et al., 1991; Vanhée et al., 1945]. In line with these observations, alveolar macrophages of asbestos exposed subjects were found to produce increased levels of TNF, IL-1 and IL-6 [Perkins et al., 1993], and alveolar macrophages from rats exposed to silica release increased amounts of TNF and IL-1 [Driscoll et al., 1990].

Previously we evaluated spontaneous and stimulated TNF release from peripheral blood monocytes of coal miners in relation to chronic coal dust exposure and CWP [Borm et al., 1988]. In a longitudinal study we were able to 
show that the risk for progression of CWP is related to individual TNF release [Borm, 1994; Schins and Borm, 1995]. In our cohort, miners with an abnormal high TNF release from monocytes stimulated with coal dust had a six-fold risk for disease progression over a five-year follow-up period [Schins and Borm, 1995].

Since peripheral blood monocytes are also important sources of TGF [Grotendorst et al., 1989] and IL-6 [Kotloff et al., 1990], we evaluated in the present study spontaneous as well as coal- and silica dust stimulated release of TGF and IL-6, in the same monocyte supernatants used to determine TNF release as previously reported [Schins and Borm, 1995]. Aims of the study were (a) to evaluate monocyte release of TGF and IL-6 in response to silica or coal dust, and (b) to study the inter-individual differences in cytokine release among nonexposed individuals versus subjects chronically exposed to coal dust, as well as in simple CWP. Overall patterns of cytokine release were analysed to evaluate their possible involvement in mineral dust exposure, and to determine which cytokines can be used as biomarkers of CWP, either independently, or in combination with TNF i.e. as "multiple markers" in coal dust induced fibrosis.

\section{Methods}

The study group consisted of 29 non-exposed individuals, 76 control miners and 28 miners with CWP. At the time of blood sampling, all coal workers had been retired [Schins et al. 1994]. An extensive questionnaire was sent to all participants in which medical complaints, medical history smoking habits, and medication were asked for. Presence and stage of CWP was evaluated by chest radiograph, scored according to I.L.O. classification rules [ILO, 1980] by an experienced panel of three physicians [Schins et al., 1994; Schins and Borm, 1995]. Miners were grouped as either "healthy coal workers" (HC, i.e. chest radiograph $=0 / 0)$ or as miners with evidence for Coal Workers Pneumoconiosis (CWP, i.e. chest radiograph $\geq 0 / 1$ ). The control group consisted of 29 , age matched, non dustexposed, male subjects. Exclusion criteria for non dust-exposed control subjects were: (I) working for more than one year in occupational settings related to chronic dust exposure (e.g. mining, cement works, ceramic industry, bakery, etc.) and (II) one or more of asthma, (chronic) bronchitis and other chronic diseases of lung, liver and kidney. Exposure parameters were used were (I) time worked underground, (II) time since first occupational exposure, (II) time since last occupational exposure, (IV) cumulative exposure to respirable coal dust and quartz content [Schins and Borm, 1995]. 
Monocyte incubations and cytokine assays

Spontaneous as well as coal- and silica dust-stimulated release of TGF and IL-6 was evaluated in the same monocyte supernatants used to determine TNF release as previously reported [Schins and Borm, 1995]. Monocytes were isolated and stimulated as described previously [Schins and Borm, 1995]. Briefly, blood (3 $\times 10 \mathrm{ml}$, EDTA-tubes) was sampled and left overnight at room temperature. Mononuclear cells were isolated by buoyant density centrifugation (LymphoprepNycomed(B), adjusted to concentrations of $0.5 \times 10^{7}$ cells $/ \mathrm{ml}$ and seeded $(0.5 \mathrm{ml})$ to tissue culture dishes (Costar TC-24) to allow adherence (repetitive incubation, agitation and washing). Adherent monocytes were stimulated with coal dust (5 $\mathrm{mg} / \mathrm{ml})$ or silica particles $(0.5 \mathrm{mg} / \mathrm{ml})$. Each individual's baseline cytokine release was measured without addition of dusts. Monocytes were incubated in RPMI culture medium containing 5\% FCS (Gibco, Life Technologies, Paisley, $\mathrm{UK})$. After 18 hours of incubation $\left(37^{\circ} \mathrm{C}, \mathrm{CO}_{2}\right)$ the cell free supernatants were stored at $-80^{\circ} \mathrm{C}$ until cytokine analysis. TNF was measured by TNF-specific sandwich-ELISA as described previously [Schins and Borm, 1995]. The detection limit of the assay was $10 \mathrm{pg} / \mathrm{ml}$. Interleukin-6 was measured by ELISA as described by Dentener [Dentener et al., 1993]. The ELISA for IL-6 has a detection limit of $10 \mathrm{pg} / \mathrm{ml}$. TGFB1 (active as well as latent forms) was measured by ELISA as described previously [Vanhée et al., 1994].

\section{Statistical evaluations}

Correlations among cytokine levels and between cytokine levels and (cumulative) exposure, age, and smoking (pack years) were evaluated by Spearman Rank correlation. Differences between subgroups were evaluated by Mann-Whitney U-test. Multiple logistic regression (MLR) was used to evaluate the significance of either spontaneous or particle-stimulated monocyte release of TNF, TGF, and IL-6 (a) in relation to exposure (i.e. non-exposed versus healthy miners), (b) in relation to pneumoconiosis (i.e. healthy miners versus miners with (CWP), and (c) in relation to (retrospective) 5-year progression of CWP. Models were evaluated upon correction for age (model a) or cumulative exposure (models $\mathrm{b}$ and $\mathrm{c}$ ). All statistical evaluations were made using SPSS 6.1 (SPSS Inc. Chicago, IL).

\section{Results}

Study group characteristics are shown in Table 10.1. Cumulative exposure was significantly higher in miners with CWP, and age was significantly higher in the non-exposed control group, compared to miners without CWP (see Table 10.1). As previously reported, coal and silica dust stimulated (but not spontaneously released) TNF levels (18 hours) were higher in miners compared to non-exposed 
subjects (see Figure 10.1 A). Silica stimulated TNF release was lower than coal dust stimulated release at the concentrations used. IL- 6 release was not different between the groups, and unlike coal dust, stimulation with silica did not cause increased IL- 6 release compared to spontaneous IL-6 release (see Figure $10.1 \mathrm{~B}$ ). Remarkably in the control coal workers, spontaneous IL-6 release significantly exceeded silica stimulated release (Mann-Whitney, $\mathrm{p}<.01$ ). In both miner groups, but not in the non-exposed individuals, coal dust induced IL-6 was significantly higher than IL-6 released in the presence of silica (Mann-Whitney, p<.01).

Table 10.1 Characteristics of the study cohort in $1992(n=133)$.

\begin{tabular}{lccc} 
& $\begin{array}{c}\text { Non-exposed } \\
\text { references }\end{array}$ & $\begin{array}{c}\text { Control } \\
\text { miners }\end{array}$ & $\begin{array}{c}\text { Miners } \\
\text { with CWP }\end{array}$ \\
\hline Age (years) & $(\mathrm{n}=29)$ & controls $(\mathrm{n}=76)$ & CWP (n=28) \\
Smoking (packyears) & $50.3(1.0) *$ & $47.7(0.6)$ & $49.9(1.1)$ \\
Cumulative dust $(\mathrm{gh} / \mathrm{m} 3)$ & $115(20)$ & $111(12)$ & $150(24)$ \\
Cumulative quartz (mgh/m3) & - & $91.1(6.4)$ & $130.9(9.3)^{* *}$ \\
Years underground & - & $4798(306)$ & $6751(461)^{* *}$ \\
Years since first exposure & - & $22.2(0.4)$ & $25.0(0.9)^{* *}$ \\
Months retired & - & $28.9(0.6)$ & $30.7(1.0)$ \\
\hline Values are & - & $46.3(2.1)$ & $53.9(2.1)$ \\
\hline
\end{tabular}

Values are mean and s.e.m.; the asterisks denote a significant difference towards the control miners group: * $\mathrm{p}<0.05$ ** $\mathrm{p}<0.01$ (Mann-Whitney)

Spontaneous as well as coal and silica dust stimulated TGF release was significantly higher in miners compared to non-exposed individuals (see Figures $10.2 \mathrm{~A}$ and 10.2 B). However at the concentrations used, TGF release from dust or silica stimulated monocytes were significantly lower than their baseline release, except in the non-exposed control group. In this group only coal dust stimulated release was significantly lower. Furthermore, and significantly only in the nonexposed group ( $p<.01$, Mann-Whitney), TGF tended to be lower in the coal dust incubations, than in the silica incubations. None of the cytokines was significantly correlated to exposure parameters or smoking, except active TGF in the silica stimulated supernatants, which was slightly higher in nonsmokers compared to smokers ( $\mathrm{p}<0.05$, Mann-Whitney). Silica stimulated TGF release tended to decrease with increasing radiological profusion (Spearman $\rho=-0.39$, $p<0.05)$. Spontaneous TNF and (total) TGF release were inversely correlated $(\rho=-$ $0.26, p<0.01)$, while TNF and IL-6 were positively related $(\rho=0.41, p<0.001)$. 
(A) TNF- $\alpha$

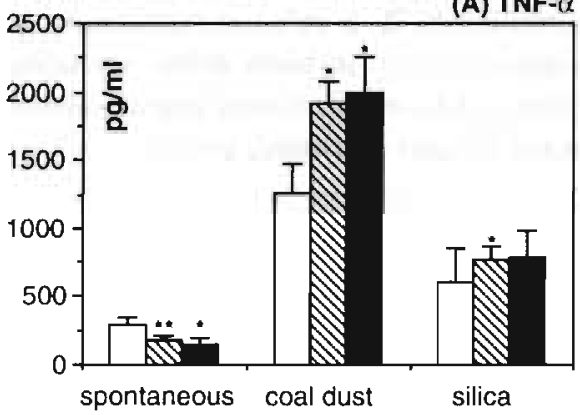

(B) IL-6

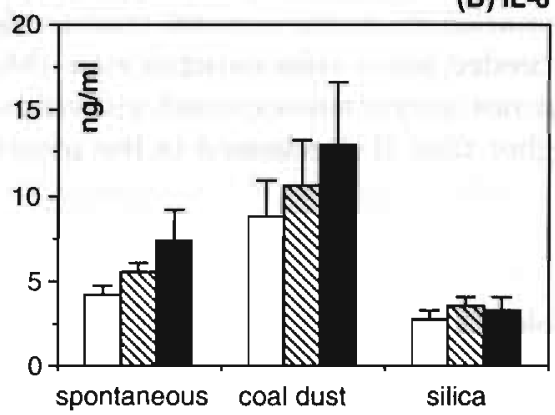

Figure 10.1. Spontaneous, coal dust stimulated, and silica stimulated release of TNF (panel A), and IL-6 (panel B) from monocytes of non-exposed references ( $n=29$, open bars), control miners ( $n=76$, hedged bars), and miners with CWP ( $n=28$, solid bars). All values are evaluated by Mann-Whitney U-test. The asterisks indicate a significant ditference versus the reference group at ${ }^{*} p<0.05$ and $* *$ $\mathrm{p}<\overline{0} .01$ respectively.

(C) TGF- $\beta$ (active)

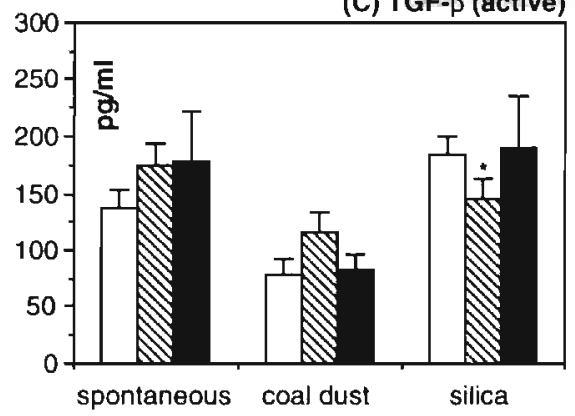

(D) TGF-B (total)

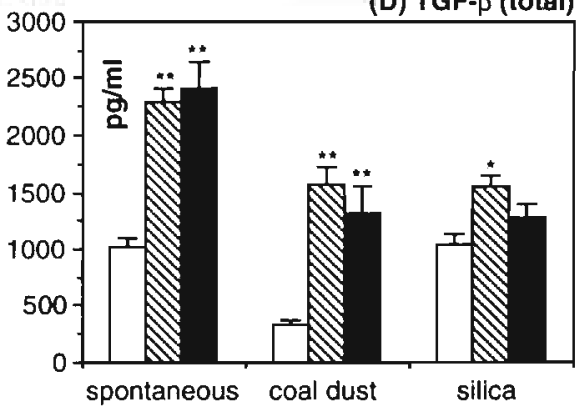

Figure 10.2. Spontaneous, coal dust stimulated, and silica stimulated release of TGF from monocytes of non-exposed references ( $n=29$, open bars), control miners ( $n=76$, hedged bars), and miners with CWP ( $n=28$, solid bars). All values are evaluated by Mann-Whitney U-test. The asterisks indicate a significant difference versus the reference group at ${ }^{*} p<0.05$ and ${ }^{* *} p<0.01$ respectively. Panel $C$ represents TGF levels without acidification of the supernatants; panel D represents TGF levels upon acidification. 
Correlations between TNF and IL-6 also reached significance in coal workers without CWP. Coal dust stimulated IL-6 release was positively correlated to TNF $(\rho=0.42, p<0.001)$ and to TGF $(\rho=0.37, p<0.001)$, and this relation was also significant in the non-exposed individuals. Remarkably, in the non-exposed individuals coal dust stimulated TNF and TGF were positively correlated $(\rho=0.55, p<0.01)$ while in the miners with CWP a significant negative correlation was observed $(\rho=-0.55, p<0.01)$. In control miners, correlation between TGF and TNF was absent.

Multiple logistic regression analyses (Table 10.2), showed that the release of TNF, IL-6 and TGF was significantly related to exposed (i.e. miners) versus nonexposed individuals as well as to pneumoconiotic versus healthy miners. Since age was higher in the non-exposed subjects (see Table 10.1), analysis was also performed upon correction for this parameter. Similarly, models were evaluated upon correction for cumulative exposure, as it was significantly higher in those with CWP (see Table 10.1), as well as those who had progression of CWP as reported previously [Schins and Borm, 1995].

Table 10.2 Multiple logistic regression analysis of spontaneous and dust stimulated monocyte release of TGF, TNF and IL-6 in relation to chronic coal dust exposure, and (progression) of CWP.

Odds

[95\% C.I.] Odds adjusted\$ [95\% C.I.]

Exposure ( $\mathrm{n}=76$ vs. $\mathrm{n}=29$ )

$\begin{array}{lcccc}\text { spontaneous } & 18.89^{*} & {[4.33-82.32]} & 27.08 * & {[6.65-110.3]} \\ \text { coal dust } & 7.00^{*} & {[1.99-24.66]} & 22.00^{*} & {[5.33-90.57]} \\ \text { silica } & 2.69 & {[0.35-20.72]} & 2.50 & {[0.65-9.57]}\end{array}$

CWP ( $n=28$ vs. $n=76)$

$\begin{array}{lcccc}\text { spontaneous } & 9.35 \# & {[0.91-95.96]} & 4.17 \# & {[0.99-17.51]} \\ \text { coal dust } & - & + & 5.36^{*} & {[1.13-25.29]} \\ \text { silica } & - & + & 5.13 * & {[1.16-22.64]}\end{array}$

Progression ( $n=17$ vs. $n=87$ ) spontaneous coal dust silica

$\begin{array}{ccc}+ & - & t \\ + & - & + \\ + & 4.36 & {[0.25-75.30]}\end{array}$

\$Odds ratio corrected for age (Exposure) or cumulative dust exposure (CWP, Progression), using multiple logistic regression; * sign at $95 \%$ C.I. ( $<<0.05)$; \# sign at $90 \%$ C.I. $(\mathrm{p}<0.10)$; tOdds ratio not calculated; empty cells 
These models were applied for spontaneous and stimulated cytokine release, and led to the Odds ratio's ( $95 \%$ confidence intervals) of the observed and predicted classification according to the models shown in Table 10.2. Spontaneous as well as coal dust stimulated patterns of cytokines release from monocytes were affected in reference miners compared to the non-exposed controls (i.e. related to chronic occupational exposure history). Furthermore, both spontaneous and dust stimulated release were related to the presence or absence of pneumoconiosis. Opposite to spontaneous release however, stimulated release was significant only upon adjustment for cumulative exposure.

\section{Discussion}

In the present study, we evaluated whether individual differences in (patterns of) cytokines released from peripheral blood monocytes, including TNF, TGF and IL-6 can be used as biomarkers in CWP. Independent analysis of the cytokines showed that TNF, TGF as well as IL-6 were affected by chronic dust exposure or pneumoconiotic disease (see Figures 10.1 and 10.2). As reported previously, coaland silica dust stimulated TNF release was higher in miners compared to nonexposed subjects [Schins and Borm, 1995]. The new data show that both spontaneous and dust stimulated release of TGF are increased in monocytes of subjects chronically exposed to coal dust. However at the concentrations used, spontaneous release of TGF from monocytes was higher than the silica and the coal dust-stimulated release. These differences may be caused by inhibitory effects of other cytokines released during the incubations in response to these dusts, or by absorption of the TGF by dust. Support for the latter explanation was obtained from preliminary experiments using incubation of recombinant human TGF in the presence of coal dust (data not shown). The release of IL-6 from unstimulated monocytes, as well as from monocytes stimulated with coal dust, was generally correlated to TNF release. Unlike TNF however, dust stimulated IL- 6 release was not increased, and spontaneous release was not decreased in (control) miners compared to non-exposed individuals. Furthermore, IL-6 release from monocytes incubated in the presence of silica did not exceed its baseline release. Bearing in mind that the silica content of the batch of coal dust (i.e. $6.4 \%$ /number or $6.2 \% /$ weight) at its concentration used (i.e. 5 $\mathrm{mg} / \mathrm{ml}$ ) did not exceed the concentration of silica used (i.e. $0.5 \mathrm{mg} / \mathrm{ml}$ ), these differences might be related to differences in the hazard of silica versus coal dust [Gosset et al., 1991].

Although differences between the non-exposed references and the (control) miners were observed, none of the cytokines was related to cumulative dust exposure in the miners. These data are not in favour of the use of monocyte release of TNF, TGF or IL-6 as exposure markers in coal miners. Furthermore, no 
relation was seen with radiological stage of CWP (conventional chest radiograph, HRCT), with the exceptions of silica stimulated TGF release and, as previously reported [Schins and Borm, 1994], coal dust stimulated TNF release, which both tended to decrease with increasing radiological stage of CWP. Finally, none of these cytokines measured was related to progression of CWP. However, it should be emphasized that present analysis was performed retrospectively, and that only prospective analysis by means of future follow-up of the present cohort will eventually elucidate the predictive value of present multiple cytokine models. Previously we have validated the release of TNF in such a prospective assay [Schins and Borm, 1995].

Based on these previous results and the present cross-sectional data we now consider combined measurement of multiple cytokines as powerful candidate markers in CWP. Indeed, multiple logistic regression analysis (see Table 10.2) clearly shows the benefits of the multiple marker interpretation of monocyte release of TGF, TNF and IL-6, in comparison to a "single marker" approach. However, the phenomenon of combined cytokine release from the same monocytes, also rises specific questions on the biological interpretation of these data. Cytokines released from the monocytes may be involved in autocrine amplification loops or in feedback mechanisms, as well as in synergisms or antagonisms with other cytokines or (soluble) receptor (ant)agonists released from the same monocytes.

The complexity of these cytokine network interactions [Elias et al., 1990] in the monocyte incubations is underscored by several observations in our study. First, in the spontaneous incubations, TNF and TGF were negatively correlated, while TNF and IL- 6 were positively correlated. A correlation between spontaneous release of TNF and IL-6 supernatants from mononuclear cells from sarcoidosis patients, but not in the controls, has been recently reported [Prior et al., 1996]. In contrast, the correlation between TNF and IL-6 reached significance in our control miners only, but neither in the non-exposed references nor in the miners with CWP. Secondly, when considering dust stimulated monocytes, TGF and TNF were positively correlated in the non-exposed individuals, inversely correlated in subjects with CWP, while in control miners correlation was absent. In line with this observation one could suggest the presence of specific interindividual differences in patterns of the relative release of TGF and TNF in relation to chronic coal dust exposure and/or resultant disease. To further evaluate this concept, individuals were classified into one out of four groups based on upper and lower tertiles of TNF and TGF levels (Table 10.3), excluding subjects with TNF and/or TGF release in the middle tertile. Age, cumulative dust exposure and smoking were not significantly different among these four groups (data not shown). Remarkably, none of the non-exposed individuals had high release of TGF, while only 3 out of 12 had high release of TNF, and none of 
the miners with CWP showed either low release or high release of both TNF and TGF at the same time. A third observation emphasizing the complexity of the cytokine networks, is the difference in spontaneous versus stimulated release of the cytokines, considering that all miners involved in this study had been retired for considerable time [Schins and Borm, 1995]. Coal dust stimulated release of TCiF and TNF were both elevated in the (retired) miners in comparison to the non-exposed individuals (see Figures 10.1 and 10.2). However, spontaneous release of TGF was approximately twice as high in the retired workers compared to non-exposed individuals, while spontaneous release of TNF was significantly lower in those formerly exposed (see Figures).

Table 10.3 Classification of non-exposed controls, reference miners, and miners with CWP versus individual release of TNF and TGF from dust stimulated monocytes

\begin{tabular}{lccccc} 
TNF/TGF & & low/low & high/low & low/high high/high \\
\hline Ninn inposed refercnces & $(n=15)$ & 12 & 3 & - & - \\
Control miners & $(n=24)$ & 2 & 6 & 9 & 7 \\
Miners with CWP & $(n=8)$ & - & 3 & 5 & - \\
\hline
\end{tabular}

\$Subjects were grouped according to upper (= "high") and lower (= "low") tertiles of cytokine reluase: Group $1=$ subjects having low TNF and low TGF release, group $2=$ subjects having high TNF and low TGF release, group 3 = subjects having low TNF and high TGF release, and group $4=$ subjects having high TNF and high TGF release.

(Ine might speculate on the meaning or role of kinetic mechanisms, or different subpopluations of peripheral blood monocytes, between retired miners versus non-exposed individuals. Although peripheral blood monocytes are a major source for newly recruited alveolar macrophages (AM), it is not known as to how far these in vitro observations play a crucial role in CWP. Remarkably however, alveolar macrophages from PMF patients spontaneously release increased levels of TNF, but lower TGF as compared to subjects with (simple) CWP [Lasalle et al., 1990; Vinhée et al., 1995]. Macrophage supernatants of those with simple CWP, but not of those with PMF, and not if incubated in the presence of anti-TGF, inhibit fibroblast proliferation in vitro [Vanhée et al., 1995]. Furthermore, time course studies in (bleomycin induced) fibrosis demonstrated a shift in TGF source from AM at early stages of fibrosis to extracellular matrix in later stages [Khalil et al., 1989], as well as changes in TNF secretion related to changes in AM subpopulations [Everson and Chandler, 1992]. In line with these observations, 
interestingly also subsets of peripheral blood monocytes are known to have considerable differences in cytokine releasing capacities [Frankenberger et al., 1996].

However, it should be realized that the observed patterns of cytokine release in the present study are also dependent on the concentrations of dusts used, as well as on the time of the incubations. In the present study, these experimental conditions (see methods section) are based on the original kinetic experiments to determine the optimal conditions regarding the release of TNF solely [Borm et al., 1988; Schins and Borm, 1995]. As such, the final concentrations of the cytokines measured in the supernatants of the unstimulated or dust stimulated monocytes, are the net result of the experimental conditions used (incubation time, concentrations of dust), but necessary also reflect inter-individual differences in kinetic response of each individuals monocytes. Irrespectively of the experimental conditions, two major observations can be made: (1) Monocytes of retired coal miners seem to be primed to release increased levels of TGF without any ex vivo challenge, while they need a specific stimulus (e.g. coal dust) to release increased TNF. (2) In vitro stimulation of monocytes of individuals (formerly) exposed to coal dust leads to an enhanced release of both TNF and TGF compared to monocytes of non-exposed references, while in those with pneumoconiotic disease, the enhanced release of either TGF or TNF dissappears.

Finally, the spontaneous TGF release measured in the present study was found to be negatively correlated to glutathione concentrations in erythrocytes, isolated from the same individuals at the same day $(r=-0.25, p=0.01, n=117)$. Moreover, we found in a prospective survey that reduced erythrocyte GSH levels measured at earlier time were a risk factor for the development of CWP [Schins et al., submitted for publication]. Since a downregulatory effect on GSH accumulation by TGF in vitro was recently reported [Arsalane et al., 1995], our findings provides indirect support for the involvement of monocyte TGF release and related red cell GSH in CWP.

In conclusion, our data show that in addition to TNF, also IL-6 and TGF play a role in coal dust exposure and/or resultant pneumoconiotic disease. Future follow-up of our study cohort will elucidate the validity of the combined evaluation of these cytokines in (ex) coal miners as susceptibility markers of CWP. 


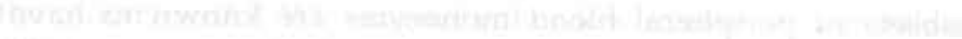

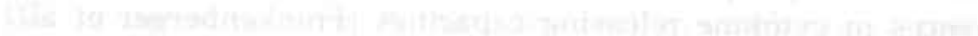

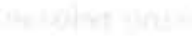

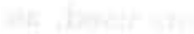

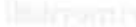

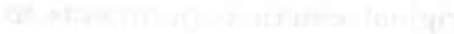

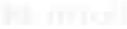

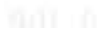


PART 3

Collagen 
ETMAT

mention 


\title{
Chapter 11
}

\section{SERUM PROCOLLAGEN TYPE III PEPTIDE IN COAL WORKERS PNEUMOCONIOSIS: A FIVE YEAR FOLLOW-UP STUDY.}

\author{
Roel PF Schins and Paul JA Borm. \\ Department of Health Risk Analysis and Toxicology, University of Limburg, \\ PO BOX 616, $6200 \mathrm{MD}$, Maastricht, the Netherlands.
}

Published in: Experimental Lung Research 1994;20:445-55.

\begin{abstract}
Several studies have suggested the use of serum type III procollagen peptide (PIIIP) as a marker in fibrotic lung disease. To test serum PIIIP as a biomarker for progression of coal workers' pneumoconiosis (CWP), a follow-up study was conducted among 156 coal miners. A five-year progression of simple CWP was determined from paired chest $\mathrm{X}$-ray readings and related to serum PIIIP measured in $1987(n=73)$ and in $1992(n=104)$. Although in eight subjects CWP had progressed, none of these subjects had abnormal levels of serum PIIP, nor were they different from non-progressed miners with regard to exposure, age or smoking habits. The results suggest that the use of serum PIIIP as a biomarker to screen for coal workers' pneumoconiosis is limited.
\end{abstract}

\section{Introduction}

A crucial biological mechanism in pulmonary fibrosis is the (chronic) activation of fibroblasts and the subsequent excessive formation and deposition of collagen fibrils [Snyder, 1988]. During early stages of pulmonary fibrosis the ratio of collagen type III to collagen type I increases at active fibrotic sites [Bateman et al., 1981]. In the search for biological markers to better predict or estimate development, outcome, or activity of disease, determination of the serum levels of the procollagen type III peptide (PIIIP) -a degradation product of the type III procollagen precursor- has been widely applied in the context of many diseases of the lung.

Several studies have established relations between PIIIP levels in serum or bronchoalveolar lavage (BAL) and several lung diseases [Low et al., 1992; Cavalleri et al., 1991; Pohl et al., 1992; Bjermer et al., 1986]; Other studies have failed to show clear data on the role of PIIIP in pulmonary diseases [Okazaki et al.,1987; Low et al., 1983; Luisetti et al., 1990]. The expression of PIIIP turns out to 
be highly dependent on the type of lung disease [Okzakai et al., 1987; Low et al., 1983; Cantin et al., 1988]. Interestingly however, within a single disease a large variation in PIIIP levels often is also present at the individual level, such as in asbestosis [Cavalleri et al., 1991; Okazaki et al., 1987], interstitial pulmonary fibrosis [Low et al., 1983; Luisetti et al., 1990], sarcoidosis [Pohl et al., 1992; Bjermer et al., 1986; Low et al., 1983; Cantin et al., 1988], farmers lung [Antinnen et al., 19861], or, for instance, in rheumatoid lung disease [Gilligan et al., 1990]. In several studies it has been suggested that serum PIIIP measurements can contribute to early diagnosis [Low et al., 1992; Cavalleri et al., 1991; Okazaki et al.,1987; Okazaki et al., 1983]. The only way to determine the role of (serum) PIIIP measurements in predicting the development or progression of a disease is through a longitudinal design. However, decent follow-up studies among subjects involved in the original cross-sectional studies [borm et al., 1992; Borm and Schins, 1993], are mostly lacking.

This article describes the results of a 5-year follow-up study of a cohort of miners designed to test procollagen type III peptide as a biological marker of early diagnosis or progression of Coal Workers Pneumoconiosis (CWP). Previously, we [Janssen et al., 1992] observed that coal miners without radiological evidence of pneumoconiosis had higher PIIIP levels than non-dust-exposed control subjects. Second, a decreasing trend in PIIIP levels was seen along with the clinical progression of pneumoconiosis (defined by chest X-ray), in line with the fact that type III collagen is mainly formed at early fibrosis. Third, and most important with regard to our follow-up design, a large proportion of subjects with abnormally high (>mean $+2 \mathrm{SD}$ of non-dust-exposed controls) PIIIP levels was observed among healthy miners and miners at early stages of CWP. It was suggested that these individuals had increased fibrotic activity, not detectable in chest X-rays, and as such might be at increased risk to develop or progress in early stage pneumoconiosis. To test this hypothesis, a 5-year follow-up study of the original cohort of coal miners was conducted to measure progression of CWP.

\section{Methods}

\section{Subjects and protocol}

Miners ( $n=156$ ) from previous studies in 1987 (Fig. 11.1) were contacted and asked to participate in the follow-up study. Chest $X$-rays were made according to the criteria of the International Labour Organisation [ILO, 1980]. Blood $(30 \mathrm{ml})$ was sampled, treated and stored at $-80^{\circ} \mathrm{C}$ until analysis of serum PIIIP levels by radioimmunoassay as described previously [Janssen et al., 1992]. Control levels of PIIIP were obtained from 29 age matched, non-dust exposed, male subjects. A complete job history of the subjects was obtained by questionnaire and personal interview. Exclusion criteria for non-dust-exposed controls were (1) working for 
more than one year in occupational settings related to chronic dust exposure (mining, cement works, ceramic industry, bakery, etc.) or (2) asthma, (chronic) bronchitis or other chronic diseases of lung, liver and kidney.

\section{Exposure history}

Although cases and control miners in our cohorts previously were matched on age and years underground, small differences in exposure might affect conclusions on the use of PIIIP as a biological marker. For this reason the occupational history from each subject was gathered. Individual dust exposure was calculated from each individuals' job exposure matrix obtained from a personal interview at follow-up and the medical file. Exposure parameters used for this study were: (1) cumulative dust exposure, (2) cumulative quartz exposure, (3) years underground, (4) years since first exposure, and (5) months retired.

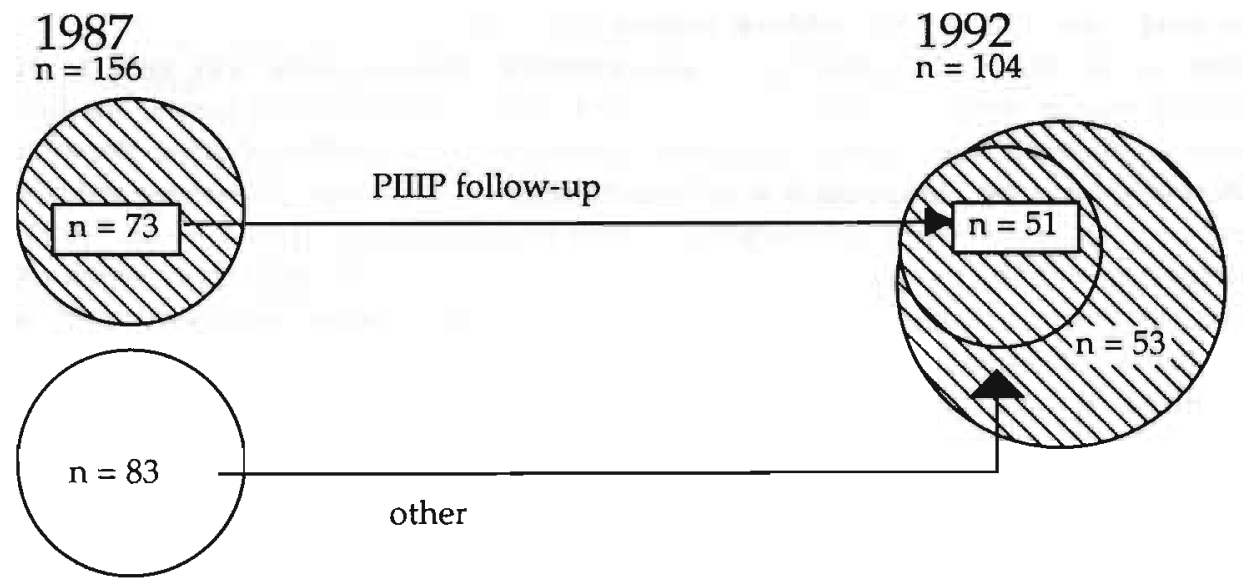

Figure 11.1. Study design and study groups. The shaded area indicates the groups in which serum PIIIP has been determined. Serum PIIIP levels in 1987 were determined in 73 miners, and in 1992 in 104 miners. The PIIIP follow-up subgroup consisted of 51 (out of 73) miners; in 53 (out of 83) miners PIIP levels were measured for the first time in 1992.

ILO classification of chest X-rays and study groups

Chest X-rays taken in 1987 and in 1992 were scored according to the classification rules of the International Labour Organisation [ILO, 1980] by an experienced panel of three physicians. Scoring was done in plenary sessions where consensus had to be reached based on individual scores. Standard ILO chest X-rays were 
used for reference to classification. The reading panel consisted of three occupational physicians; two of them were seeing more than 3000 chest X-rays a year, and one was a trained reader but had not been active as such for the last three years. Two of the three readers were also involved in our previous studies. Based on the ILO scores, the dust-exposed cohort was subdivided into 6 groups: $0 / 0,0 / 1, \mathrm{I}(=1 / 0,1 / 1,1 / 2), \mathrm{II}(=2 / 1,2 / 2,2 / 3), \mathrm{II}(=3 / 2,3 / 3)$, and IV $(=3 /+, \mathrm{PMF})$. Chest $X$-rays were scored in two plenary sessions. In the first session all $X$-rays i.e. of 1987 and 1992 were scored randomized blind. In a second session individuals 1987 and $1992 \mathrm{X}$-rays photographs were scored pairedwise. The randomized blind reading sessions were used to determine intra-reader variability. However, as a gold standard for 5-year progression or new development of CWP, the outcome from the pairedwise reading session of each individual's chest $X$-ray of 1987 and 1992 was used. Readers were allowed to consult medical files and additional Xrays from other years to facilitate diagnosis of CWP, if consensus could not be reached unambiguously.

\section{Smoking, medication and medical history}

Prior to the medical consultation, an extensive questionnaire was sent to all participants in which questions were asked about medical complaints, medical history, smoking habits and medication. Answers were verified during personal interviews and by comparison with questionnaires from our preceding studies. Smoking amounts were calculated as described elsewhere [Jorna et al., 1994]. Furthermore, subjects were subdivided into current, ever, and never smokers. With respect to medication and medical history, subjects were classified based on the presence or absence of any medication during 3 weeks prior to blood sampling, or according to presence or absence of medical complaints.

\section{Statistical evaluations}

Five-year changes in PIIIP were tested by paired $t$ test. Correlations between PIIIP levels of 1987 and 1992 versus exposure, age, smoking, etc., were evaluated by means of multiple linear regression. Unpaired differences between 1987 and 1992 (sub) groups were tested by the Mann Whitney $U$ test. Differences between miners without or with CWP and non-dust-exposed controls, and with respect to medication, medical history, smoking were tested by the Kruskall Wallis test and ANOVA. Odds ratio's and confidence intervals were calculated to determine the significance of PIIIP levels in predicting 5-year progression of pneumoconiosis. All statistical evaluations were made using STATGRAPHICS (version 6.0). 


\section{Results}

One hundred and four coal miners participated in our-follow-up. Demographic characteristics of these study groups are given in Table 11.1 and Figure 11.1. For 51 miners we had 1987 serum type III procollagen levels from our original study [Janssen et al., 1992]. The other group of 53 miners were also screened in 1987, but in a different survey. When the population parameters of both the original and the follow-up groups were checked, no significant selection on age, smoking habits, medication or years underground was observed. No selection bias with regard to pneumoconiotic stage (i.e., $1987 \mathrm{X}$-ray) was present.

Table 11.1 Serum procollagen type III peptide (PIIP) levels, age, exposure, smoking, and the five-year progression of pneumoconiosis in PIIP subcohort $(n=51)$.

\begin{tabular}{lcccccc} 
& \multicolumn{2}{c}{ ILO 1987 $=0 / 0$} & \multicolumn{2}{c}{ ILO 1987 $\geq 0 / 1$} & \multicolumn{2}{c}{ All miners } \\
\hline Five-year progression & $n 0$ & $y e s$ & $n o$ & yes & no & yes \\
\multicolumn{1}{c}{$\mathrm{n}$} & 39 & 2 & 4 & 6 & 43 & 8 \\
1987 PIIIP $(\mathrm{ng} / \mathrm{ml})$ & $61.26(2.3)$ & $70.45(11.1)$ & $63.93(3.0)$ & $52.02(4.6)$ & $61.51(2.1)$ & $56.63(5.0)$ \\
age (years) & $47.2(0.7)$ & $47.5(5.5)$ & $49.5(2.0)$ & $54.0(1.2)$ & $47.4(0.7)$ & $52.4(1.7)^{*}$ \\
exposure: & & & & & & \\
cum. dust (g.h. m $\left.{ }^{-3}\right)$ & $91.6(9.4)$ & $155.0(45.0)$ & $143.0(5.1)$ & $110.0(31.2)$ & $96.4(8.8)$ & $121.3(25.4)$ \\
years underground & $22.5(0.7)$ & $26.0(6.0)$ & $26.5(1.6)$ & $27.0(1.8)$ & $22.8(0.6)$ & $26.8(2.0) \#$ \\
years since first exposure $29.5(0.9)$ & $29.5(7.5)$ & $33.3(2.1)$ & $35.2(1.5)$ & $29.9(0.8)$ & $33.8(2.0)$
\end{tabular}

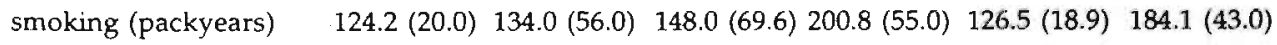

Values are mean and standard error. ILO $=1987$ chest radiograph according to ILO criteria.

${ }^{*} \mathrm{p}=0.012$, and \# $\mathrm{p}=0.035$, significantly different from non-progressed (in whole miners group)

\section{Retirement}

During the follow-up period all miners had left active mining. Occupational exposure had ended for at least 4 years in half of our miners group and for at least 2 years in more than $85 \%$ of the miners. No selective loss to follow-up had taken place with regard to retirement (Figure 11.2). The average age of retirement in this group was $44.7 \pm 5.1$ (mean \pm SD). 


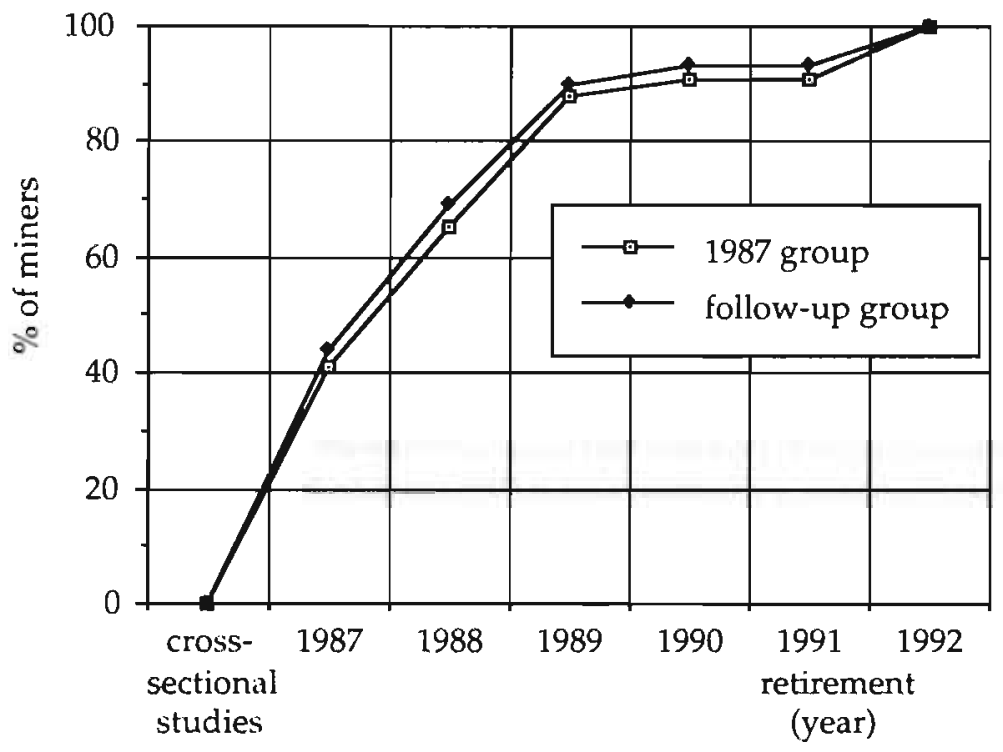

Figure 11.2. Retirement in original cohort $(n=156)$ and follow-up group $(n=104)$ during the five year follow-up interval.

PIIIP measurements of 1987 and 1992

Paired testing of the PIIIP data of 1987 and 1992 in 51 coal miners revealed a significant difference $(\mathrm{p}<.001)$ between serum PIIIP levels in these two years. Also the mean, mode, minimum and maximum of all 1992 values $(n=104)$ were lower than all 1987 values $(n=71)$. In Figure 11.3A, serum PIIIP levels of the various groups are compared, as determined in 1987 and 1992. In 1992, mean PIIIP levels of miners without radiological evidence of CWP, and those of miners of group I and group II were significantly lower than 1987 ( $p<.01)$. Significantly lower PIIIP levels were also observed for the non-dust-exposed control group in 1992 ( $p<.01$, Figure 11.3A). Since these differences might be a consequence of some major modifications in the radioimmunoassay of the procollagen peptides, serum PIIIP values were also calculated as a percentage of the non-dust-exposed control levels (Figure 11.3B). After this correction, differences between serum PIIIP levels within the different categories remained apparent, although they were significant only for the control miners group. 
Figure 11.3. Comparison of serum procollagen type III peptide (PIIIP) between the 1987 and 1992 study groups. On the horizontal axis subgroups are $0 / 0,0 / 1$, group I $(1 / 0,1 / 1,1 / 2)$, group II $(2 / 1$, $2 / 2,2 / 3)$, group III $(3 / 2,3 / 3)$, group IV (progressive massive fibrosis), and non-dust-exposed controls (ndc).

Figure $A$ represents absolute values $(\mathrm{ng} / \mathrm{ml})$; in figure $B$ values are expressed as a percentage of the non-dustexposed controls. Bars represent mean and standard deviations. The asterisk denotes a significant difference $(p<.05)$ between subgroups of 1987 (shaded bars) and 1992 (open bars).
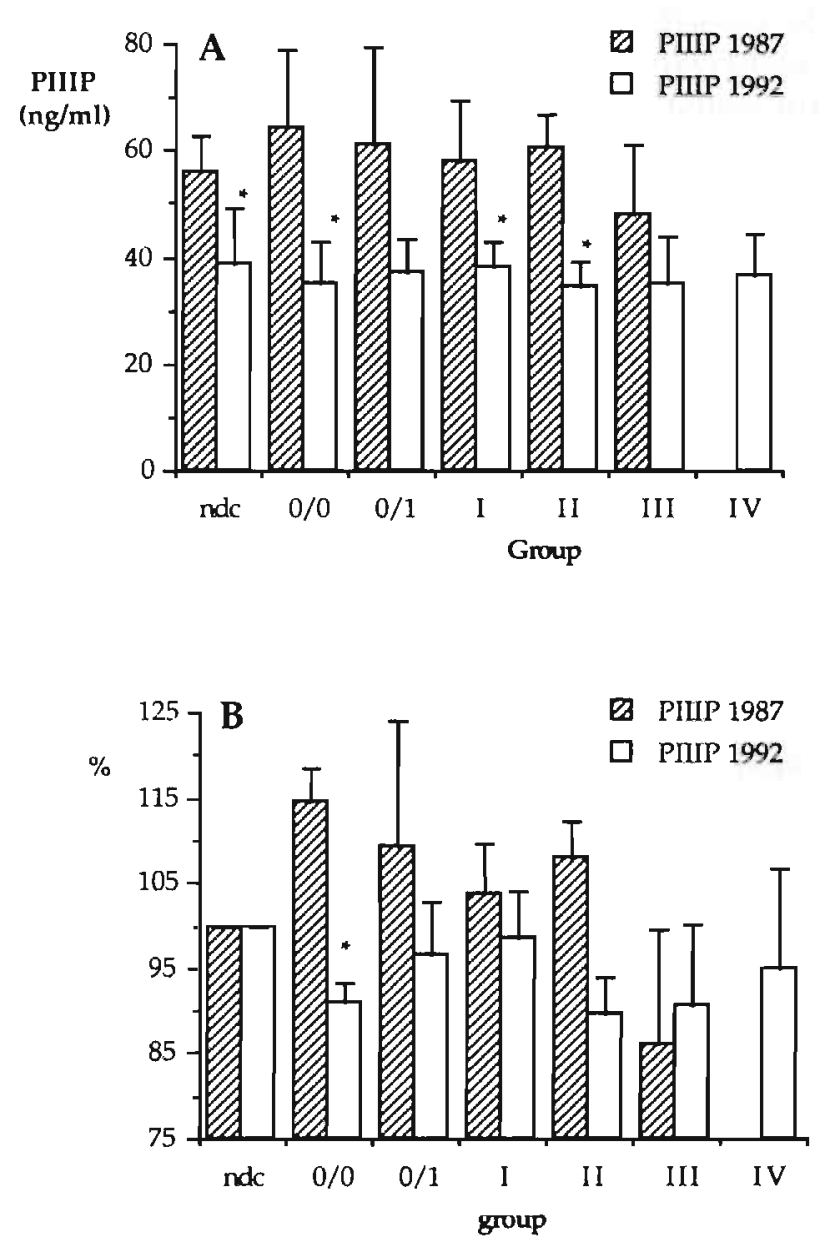

Unlike in our previous findings, PIIIP levels were not significantly increased in coal miners versus their non-dust-exposed controls. Furthermore, the downward trend of PIIIP levels versus pneumoconiotic stage (defined by chest $X$ ray) was not observed at present (figure 11.3A and 11.3B). Moreover, all subjects now have normal serum PIIIP levels $(<$ mean +2 SD of the non-dust-exposed control group). Evidently a poor relation is present between serum PIIIP levels at the two time points measured, demonstrated by a poor correlation in the total 
group ( $n=51$ ) and a significant negative correlation between 1987 and 1992 levels in the CWP group (see table 11.2).

Table 11.2 Correlation of PIIP-data 1987 versus $1992(n=51)$.

\begin{tabular}{|c|c|c|}
\hline group & $\mathbf{r}$ & p-value \\
\hline all miners & 0.12 & 0.39 \\
\hline ILO $1987=0 / 0(41)$ & 0.21 & 0.20 \\
\hline ILO $1987 \geq 0 / 1(10)$ & -0.69 & 0.02 \\
\hline
\end{tabular}

Serum PIIIP and progression of CWP

In our PIIIP follow-up cohort ( $n=51)$, no correlation was observed between PIIIP levels and smoking (packyears), age, or any parameter of exposure. Moreover, serum PIIIP levels were not significantly different with respect to medication, medical history, or past and current smoking habits ( $n=104)$. Interestingly, a significant correlation was observed between serum PIIIP and weight $(r=.26$, $\mathrm{n}=104, \mathrm{p}<.01$ ), but none of the above parameters were correlated to serum PIIIP upon correction for body weight $\left(\mathrm{ng} / \mathrm{mL} \mathrm{kg}^{-1}\right)$.

Despite retirement, a significant proportion (16\%) of this cohort showed progression of CWP during the 5-year follow-up period. Four out of 80 healthy coal miners contracted CWP, and 13 out of 25 cases CWP did progress. In the original cohort of miners involved in the procollagen study of 1987, 2 miners newly developed pneumoconiosis and in 6 out of 10 subjects already existing pneumoconiosis progressed. However, none of these subjects had abnormal levels of procollagen $\left(\mathrm{ng} / \mathrm{ml}, \mathrm{ng} / \mathrm{mL} \mathrm{kg}^{-1}\right)$ in 1987 or in 1992. Furthermore, in the PIIIP follow-up cohort $(n=51)$, 5-year changes in pneumoconiosis were not related to original procollagen levels measured in 1987 (Table 11.1). A multiple regression of serum PIIIP of 1987 versus 1992, exposure and age revealed no significant correlations and therefore excludes bias from these parameters on serum PIIIP levels. There was also no difference in exposure, age or smoking habits between miners without and miners with progression of coal workers pneumoconiosis (Table 11.1). 


\section{Discussion}

Nowadays, coal workers' pneumoconiosis (CWP) has become a disease of delayed onset owing to a more efficient technological and medical prevention. This epidemiological evolution necessitates postprofessional medical follow-up of coal miners [François et al., 1988]. In the UK simple pneumoconiosis has become more frequent among men who have left industry than in the active work force [Soutar et al., 1986]. In the Lorraine mining region in France $82 \%$ of the 1986 incidence of CWP was observed in retired coal workers [Mahieu, 1990]. Complementary to these epidemiological findings, comprehensive biological explanations for the individual development and progression in CWP still need to be elucidated. Since this entire cohort had retired from active mining since first surveyed, our data on progression of CWP related to serum PIIIP might as such be useful in the postprofessional survey of coal miners. Despite retirement, a significant proportion $(16 \%)$ of the cohort showed progression of CWP. However, all miners of this cohort previously had been selected on long-term and high occupational exposure, and as such do not reflect progression rate in the general population of retired miners in the Kempen mining region in Belgium.

With respect to the determination of serum PIIIP, our previous findings in active miners [Janssen et al., 1992] were not confirmed, since this time: (1) no relationship between CWP stage and PIIIP levels were observed and (2) mean serum PIIIP levels were not significantly elevated in (retired) coal miners versus their non-dust-exposed reference group. Moreover, (3) at present no individuals showing abnormal high levels of serum PIIIP, i.e., out of the normal range (2 SD above mean) of the non-dust-exposed controls, were seen. One might attribute this to the retirement of the miners and the cessation of dust exposure. However, since no relation between the decrease in serum PIIIP and period of retirement was present (data not shown), this is unlikely to be a plausible explanation. Differences between the two non-dust-exposed control groups in both studies only strengthen the discrepancies: Unlike in our previous study, in which anonymous subjects were randomly selected from the University Hospital blood bank, in the present study we excluded subjects with occupational dust exposure and/or medical treatment and the presence of fibrotic lesions.

This 5-year follow-up study does not forward the use of serum PIIIP measurement to predict either development or progression of CWP. The relative risk for progression of a miner showing an abnormal PIIP level in 1987, as well as the calculated odds ratio of the 1992 PIIIP levels for progressed subjects versus non-progressed subjects, are both below 1, and rather suggest a tendency toward increased risk for coal miners with normal PIIIP levels. Our results are in contrast to a five year follow-up study in sarcoidosis patients [Pohl et al., 1992], where serum PIIIP levels were found to be a very good predictor of disease 
progression. One should keep in mind that sarcoidosis, in contrast to CWP, is characterized by a striking interstitial fibrosis and has a completely different origin [Snyder, 1988; Luisetti et al., 1990]. However, in an other study in sarcoidosis patients, serum PIIIP failed to predict disease prognosis [Luisetti et al., 1990]. We suggest that the types of disease and variations in fibrotic activity within one type of lung disease, reflecting moments of increased inflammation, tissue damage, wound healing, and collagen turnover, affect serum PIIIP levels. Therefore, serum determinations do not necessarily reflect the stage of disease. Perhaps serum PIIP is a marker of increased fibrotic activity, but then in this cohort the individuals were not at higher risk for progression of CWP. Unfortunately, this cohort comprised only retired miners and therefore discrimination between cessation of exposure and time variation as the two possible causes of the lower serum levels in 1992 was not possible. Data on both the effect of exposure and time variation in serum PIIIP are scarce and therefore application of this measurement as a diagnostic marker in postoccupational screening in CWP and other fibrotic lung disorders should be done with the utmost care.

Acknowledgements. The authors thank all coal miners and the Kempense Steenkoolmijnen NV Belgium for their willingness to cooperate in this study; Marc van Sprundel (PhD,MD), Luc Linaerts (MD), and Luc Mariën (MD) for reading the chest X-rays; Dr. Simons of HOECHST AG Germany for performing the PIIIP radioimmunoassay. This study was supported by Grant $7263 / 03 / 092$ of the European Community for Steel \& Coal. 


\title{
Chapter 12
}

\section{EVALUATION OF SERUM TYPE III PROCOLLAGEN PEPTIDE AS AN EXPOSURE MARKER IN RETIRED COAL WORKERS.}

\author{
Roel P F Schins ${ }^{1}$, Rob J S Lamers ${ }^{2}$, Bernard Préat ${ }^{3}$, and Paul J A Borm. \\ 1 Department of Health Risk Analysis and Toxicology, University of Limburg, \\ PO BOX 616, 6200MD Maastricht, the Netherlands. \\ 2 Department of Diagnostic Radiology, Academic Hospital Maastricht, \\ PO BOX 5800, 6202AZ Maastricht, the Netherlands. \\ 3 I.R.E.A. Environmental and Occupational Hygiene Division, \\ Kempische Steenweg 555, 3500 Hasselt, Belgium.
}

Published in: International Archives of Occupational and Environmental Health 1995;66:413-9.

\begin{abstract}
Serum type III procollagen peptide (PIIIP), a degradation product of the type III collagen precursor has been put forward as an exposure marker for mineral dust. We evaluated PIIIP levels as a marker of exposure to and effects of coal dust in retired coal miners $(n=104)$. To this end: (a) the individual cumulative dust exposure was calculated from job-exposure matrices, and (b) in addition to a routine chest radiograph (CR) of all miners according to the criteria of the International Labour Organisation (ILO), a subgroup $(n=46)$ was screened by high resolution computed tomography (HRCT). Profusion score (CR and HRCT) tended to increase with cumulative dust exposure, even in the absence of $C R$ evidence for pneumoconiosis (i.e. $C R \leq 0 / 1, n=35$ ). In contrast to our previous findings in active miners, PIIIP levels were not increased in miners compared with non dust-exposed controls ( $\Omega=29$ ), and no differences were observed between miners without (ILO $=0 / 0$ ) and miners with coal workers' pneumoconiosis (CWP; ILO $\geq 0 / 1$ ). No trend in PIIIP versus pneumoconiosis stage was present, either by CR or by the more sensitive HRCT score. PIIIP was also unrelated to any lung function parameter (FEV FVC, impedance, diffusion capacity). Age, medication, medical history and smoking habits had no significant effect on PIIIP levels. In the miners with CWP (i.e. ILO $>0 / 0$, $\mathrm{n}=28$ ) a significant negative correlation was present between PIIIP values and (log) cumulative dust exposure. This decrease in serum PIIIP levels with increasing cumulative exposure may be due to chronic adaptive changes in type III collagen deposition and/or breakdown. Other relations between exposure and PIIIP were not observed. In conclusion, the present findings do not support the use of serum type III procollagen peptide as a marker of exposure to and (early) interstitial or respiratory effects of coal dust.
\end{abstract}




\section{Introduction}

Serum or bronchoalveolar lavage levels of the type IIII procollagen peptide (PIIIP) have been studied in relation to several lung diseases, including asbestosis [Okazaki et al. 1987; Cavalleri et al. 1988; Cavalleri et al. 1991], idiopathic pulmonary fibrosis [Low et al. 1983; Cantin et al. 1988; Luisetti and Bulgheroni 1990], sarcoidosis [Bjermer et al. 1986; Pohl et al. 1992], farmer's lung [Anttinen et al. 1986], and rheumatoid lung disease [Gilligan et al. 1990]. In spite of major differences in the morphology, pathological observations, and clinical course of many of these diseases, the levels of serum (or bronchoalveolar lavage) PIIIP are believed to reflect the current activity of an on-going fibrotic disease process, characterized by the deposition of collagen fibrils at fibrotic sites [Gilligan et al. 1990; Pohl et al. 1992; Low et al. 1992].

Previously we found [Janssen et al. 1992] that serum PIIIP levels were increased in coal miners as compared with non dust-exposed controls and we observed a relationship between PIIIP level and stage of coal workers' pneumoconiosis (CWP) as defined by chest radiograph (CR) according to the criteria of the International Labour Organization [ILO 1980]. We, therefore suggested that routine measurements of PIIIP in serum could be applied for early diagnosis of CWP [Borm et al. 1992].

Based on highly significant correlations repeatedly observed between asbestos exposure and serum PIIIP in asbestos exposed workers [Cavalleri et al. 1988, 1991], PIIIP measurements have recently been forwarded as a valid tool in occupational monitoring of subjects with mineral dust exposure [Becklake et al. 1993]. In order to evaluate PIIIP as a marker of exposure to and effects [Schulte and Perera 1993] of coal dust, the original cohort of coal miners (meanwhile all retired) were recruited for a five-year follow-up study. In all miners serum PIIIP was determined, new CRs were obtained, lung function was determined by spirometry and impedance measurement, individual cumulative exposure was estimated and a subgroup was screened using high resolution computed tomography (HRCT). This follow-up showed that a significant proportion of our miners, all previously heavily exposed, progressed in CWP in spite of retirement [Schins et al. 1994]. Prospective analysis of the data however, showed that the initial PIIIP measurements were not predictive of the 5-year development or progression of pneumoconiosis [Schins and Borm, 1994]. In the present paper serum PIIIP is evaluated as an exposure marker to coal mine dust and is also compared to several indices of interstitial and respiratory effects of coal dust. 


\section{Subjects and methods}

\section{Subjects}

Retired coal miners $(n=104)$ from a combined cohort of three previous studies [Borm et al. 1988; Engelen et al. 1990; Janssen et al. 1992] among miners from the Belgian coal mining company "Kempense Steenkoolmijnen" participated. Written informed consent was obtained from each worker and after completion of a questionnaire (see below), blood $(10 \mathrm{ml})$ was sampled, lung function was determined (spirometry, impedance) and CRs were made. Blood samples were obtained and treated as described previously [Engelen et al. 1990] and serum was stored at $-80^{\circ} \mathrm{C}$ until analysis of PIIIP. Serum levels of PIIIP were determined by the radioimmunoassay method originally developed by Rhode and colleagues, as described previously [Rhode et al. 1979; Janssen et al. 1992]. PIIP levels were determined in all miners $(n=104)$ and in 29 male, age matched, non dust-exposed controls. On a voluntary basis, HRCT slices were made in a subgroup of 46 miners. Smoking habits, medication and medical history were derived from each subject as described previously [Jorna et al. 1994a; Schins and Borm 1994].

\section{$C R$ and HRCT}

CR were taken and scored according to the criteria of the ILO [1980]. Scoring was done as described previously [Schins and Borm 1994; Schins et al. 1994]. Miners with an ILO profusion score $0 / 0$ were considered to be "healthy coal workers" (HCWs), and miners with an ILO category $\geq 0 / 1$ as miners with CWP.

HRCT slices were made and scored as described previously in a subgroup of 46 miners [Lamers et al. 1994]. Since collagen type III is believed to be mainly involved at early stages of active interstitial fibrosis [Bateman et al. 1981] we focused our attention on the relationship between serum PIIP levels and HRCT in miners without the classic-diagnostic evidence for CWP. Therefore, the HRCT subgroup was divided on the basis of their CR score into miners without CWP or with an early/doubtful stage of CWP (i.e. $C R=0 / 0$ and $0 / 1$ ) versus miners with definite evidence for CWP (i.e. CR $\geq 1 / 0$ ).

\section{Exposure}

Considering the aim of the study, much emphasis was placed on a good estimation of exposure and several parameters were used, i.e. (a) time worked underground, (b) time since first occupational exposure, (c) time since last occupational exposure, (d) cumulative exposure to respirable coal dust and quartz content. Years exposed, time since first exposure and time elapsed since last occupational exposure were determined from the medical/personel file and a personal interview at follow-up. 
Exposure years were determined from the complete job history obtained from each subject and defined as the total number of years in which a subject worked underground in a coal mine. Long term absenteeism (e.g. due to disease or special leave) as well as interim job changes were taken into account. Years since first exposure were defined as the interval between the year in which a miner started working underground and the time of our study. Time from last exposure was the interval between retirement and our follow-up in months. Job exposure matrices were used to estimate each individual's (respirable) cumulative dust and quartz exposure in gram $x$ hours per cubic meter of sampled air, as described elsewhere [Jorna et al. 1994a].

\section{Lung function}

Lung function (spirometry, impedance) was performed in all coal miners as described previously [Jorna et al. 1994a]. Flow-volume parameters used in this study are $\mathrm{FEV}_{1}$ (forced expiratory volume during first second), FVC (forced vital capacity), and the ratio $\mathrm{FEV}_{1} / \mathrm{FVC}$. Airflow limitation was defined as an $\mathrm{FEV}_{1}$ less than $80 \%$ predicted value. Impedance parameters used are the resistance at 8 and at $28 \mathrm{~Hz}(\mathrm{R} 8 \mathrm{~Hz}, \mathrm{R} 28 \mathrm{~Hz})$ and the resonant frequency $\left(f_{0}\right)$. In the subgroup screened for HRCT ( $n=46)$, spirometry was repeated, and supplemented with TLC (total lung capacity) and DLCO (diffusion capacity, using carbon monoxide transfer factor), as described elsewhere [Lamers et al. 1994].

\section{Statistical evaluations}

Statistical evaluation was made using STATGRAPHICS version 6. Correlations between PIIIP levels and exposure, age, smoking and lung function parameters were evaluated by means of (multiple) linear regression. Differences between miners without or with CWP and non-dust-exposed, and with respect to medication, medical history, and smoking were tested by the Mann-Whitney Utest and the Student- $t$ test (ANOVA). Trend analysis between pneumoconiosis stage (CR, HRCT) and PIIIP, exposure or other variables were peformed by means of the Spearman's rank correlation procedure. For this purpose, profusion scores were converted to a scale from 0 to 11 for $C R$ and 0 to 80 for HRCT.

\section{Results}

\section{Radiology outcome}

The calculated cumulative exposure was bimodally distributed, due to significantly higher exposure among miners with CWP $(p<0.01$, Mann-Whitney test), as shown in figure 12.1. The outcome of radiology was significantly related to cumulative exposure, whether based on $C R$ ( $n=104, p<0.001$; Spearman's 
$\rho=0.34)$ or on HRCT $(n=46, p<0.005 ; \rho=0.44)$ analysis. Years underground also correlated with CR-scored pneumoconiosis $(p=0.29, p<0 .(005, n=104)$, and to a lesser degree with HRCT-scored pneumoconiosis ( $r=0.36, p<0.02, n=46$ ). Interestingly, a correlation was also present between years since first exposure and HRCT-defined lesions $(r=0.38, p<0.02, n=46)$, while a similar relation for years since first exposure and CR-scored pneumoconiosis was not present.

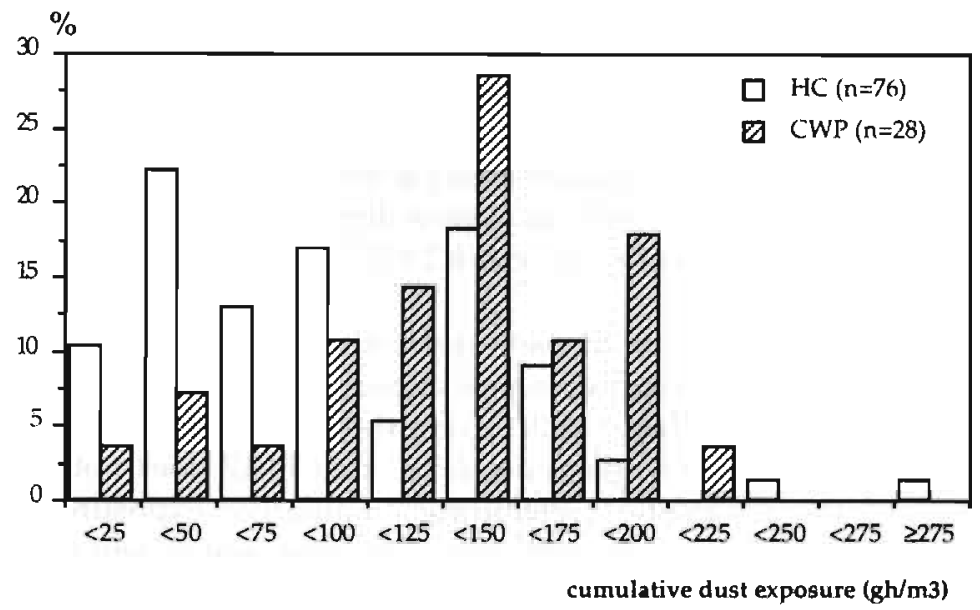

Figure 12.1 Frequency distribution of cumulative dust exposure in reference miners $(\mathrm{HC})$ and miners with CWP. The cumulative exposure was normally distributed in the miners with CWP (CR $\geq 0 / 1$, $\left.n=28, c^{2}=1.47 ; p>0.2\right)$, but not in the miners without $C W P\left(C R<0 / 1, n=76, c^{2}=16.7 ; p<0.01\right)$.

Table 12.1 compares the classification of miners according to CR versus HRCT. Although visual score of HRCT correlated significantly with CR-score $(\rho=0.53$, $\mathrm{n}=46, \mathrm{p}<0.001$ ), the data also demonstrate that small opacities are better visible in HRCT slices. About $50 \%$ of the miners (16 out of 35 ) with CR score $0 / 0$ or $0 / 1$ had a negligible number of lesions (score <5) on their HRCT slices. However, the other miners without $C R$ evidence for pneumoconiosis had a large number of small opacities only detectable by HRCT. These observations are in line with findings of others [Bégin et al. 1990; Remi-Jardin et al. 1990; Vermeire 1993] and clearly demonstrate that HRCT permits a gradation of (mild) pneumoconiosis in "CR-normal" miners, as a consequence of the higher resolution of the method. In subjects with $C R$ evidence for pneumoconiosis, CR and HRCT score are usually well in line. 
Evaluation of serum PIIIP as effect or exposure marker

Serum PIIIP values ranged from 18.3 to $81.8 \mathrm{ng} / \mathrm{ml}$, with a mean ( \pm standard error) of $36.0 \pm 0.72 \mathrm{ng} / \mathrm{ml}$ ( $n=133$; all miners plus non dust-exposed controls). Medication, medical history or past and current smoking habits had no significant effect on PIIIP levels and they were also not associated with age or any parameter of exposure. Furthermore, no relation between PIIIP and time of retirement was apparent. Serum PIIIP levels were not significantly different between miners without pneumoconiosis $(n=76 ; 35.1 \pm 0.9 \mathrm{ng} / \mathrm{ml})$ and those with pneumoconiosis $(n=28 ; 36.2 \pm 1.2 \mathrm{ng} / \mathrm{ml})$, nor was there any difference form the non-dust-exposed controls $(n=29 ; 38.6 \pm 2.0 \mathrm{ng} / \mathrm{ml})$. No significant correlations were found between serum PIIIP and any of the lung function parameters or radiographic score either by $C R(n=104, p>0.5)$ or HRCT $(n=46$, $p>0.9$ ). Furthermore, mean ( \pm standard error) levels of serum type III procollagen were not different for miners without airflow limitation $(n=92,35.1 \pm 0.8)$ and miners with airflow limitation $(n=10,36.1 \pm 2.9)$.

Subsequently, several indices of both interstitial (CR, HRCT, DLCO) and respiratory (spirometry, impedance) effects of coal dust exposure were related to serum type III procollagen (Table 12.2). Using the mean serum PIIIP value of all miners as a cut-off point, this operation shows that PIIIP does not discriminate between any of the parameters, including cumulative exposure. Moreover, linear correlations between serum PIIIP and exposure, age or smoking amounts (Table 12.3) showed that serum PIIIP levels were not related to years underground or to years since first exposure. Cumulative dust exposure was not related to serum PIIIP in miners without CWP, but interestingly in the CWP group a significant negative correlation was present between cumulative dust exposure and PIIIP values (Figure 12.2).

Table 12.1 Pneumoconiosis stage by chest radiograph (CR) and HRCT score $(n=46)$.

\begin{tabular}{|c|c|c|c|c|c|c|c|}
\hline \multicolumn{2}{|c|}{ HRCT \# } & \multirow{2}{*}{$\frac{<5}{14}$} & \multirow{2}{*}{$\frac{5-10}{9}$} & \multirow{2}{*}{$\frac{10-15}{2}$} & \multirow{2}{*}{$\frac{15-20}{5}$} & \multirow{2}{*}{$\frac{20-30}{2}$} & \multirow{2}{*}{$\frac{\geq 30}{1}$} \\
\hline CR* & $0 / 0$ & & & & & & \\
\hline & $0 / 1$ & 2 & & & & & \\
\hline & $1 / 0,1 / 1,1 / 2$ & & 1 & 1 & & 1 & \\
\hline & $2 / 1,2 / 2,2 / 3$ & & & & 1 & 3 & \\
\hline & $3 / 2,3 / 3,3 /+$ & & & & & 1 & 3 \\
\hline
\end{tabular}

\#, * See materials and methods section for details of classification 
Table 12.2 Demographic characteristics, lung function and radiology in retired miners when classified according to their serum PIIIP levels.

\begin{tabular}{|c|c|c|c|}
\hline Parametex** & Unit & PIIIP * $<$ mean & PIIIP $\geq$ mean \\
\hline & & $(n=55)$ & $(n=49)$ \\
\hline Age & $(y)$ & $48.1 \pm 0.7$ & $48.7 \pm 0.8$ \\
\hline Cumulative dust & $(\mathrm{gh} / \mathrm{m} 3)$ & $97.6 \pm 7.2$ & $105.9 \pm 8.8$ \\
\hline Smoking & (packyears) & $127.3 \pm 14.1$ & $115.3 \pm 17.6$ \\
\hline $\mathrm{FEV}_{1}$ & (L) & $3.49 \pm 0.10$ & $3.64 \pm 0.10$ \\
\hline $\mathrm{FEV}_{1}$ & $(\%)$ & $102.1 \pm 2.4$ & $105.0 \pm 2.3$ \\
\hline $\mathrm{FEV}_{1}<80 \%$ predicted & & 5 & 5 \\
\hline FVC & (L) & $4.62 \pm 0.11$ & $4.70 \pm 0.12$ \\
\hline FVC & $(\%)$ & $110.5 \pm 2.1$ & $111.1 \pm 2.0$ \\
\hline $\mathrm{FEV}_{1} / \mathrm{FVC}$ & & $0.75 \pm 0.01$ & $0.77 \pm 0.01$ \\
\hline $\mathrm{R} 8 \mathrm{~Hz}$ & $(\mathrm{hPa} \cdot \mathrm{s} / \mathrm{L})$ & $2.66 \pm 0.13$ & $2.51 \pm 0.16$ \\
\hline $\mathrm{R} 28 \mathrm{~Hz}$ & $(\mathrm{hPa} \cdot \mathrm{s} / \mathrm{L})$ & $2.46 \pm 0.09$ & $2.54 \pm 0.11$ \\
\hline$f_{0}$ & $(\mathrm{~Hz})$ & $11.0 \pm 0.6$ & $10.0 \pm 0.5$ \\
\hline \multirow[t]{6}{*}{ CR score } & $0 / 0$ & $\because$ & 36 \\
\hline & $0 / 1$ & 3 & 4 \\
\hline & $1 / 0,1 / 1,1 / 2$ & 1 & 4 \\
\hline & $2 / 1,2 / 2,2 / 3$ & i & 2 \\
\hline & $3 / 2,3 / 3,3 /+$ & 5 & 3 \\
\hline & & $(n=23)$ & $(n=23)$ \\
\hline TLC & $(\%)$ & $102.0 \pm 2.5$ & $99.5 \pm 2.5$ \\
\hline DLCO & $(\%)$ & $110.2 \pm 5.4$ & $116.7 \pm 5.5$ \\
\hline \multirow[t]{5}{*}{ HRCT score } & $<5$ & 8 & 8 \\
\hline & $5-10$ & 5 & 5 \\
\hline & $10-15$ & 2 & 1 \\
\hline & $15-20$ & 3 & 3 \\
\hline & $>20$ & 5 & 6 \\
\hline
\end{tabular}

* The mean PIIIP value $(35.3 \mathrm{ng} / \mathrm{ml})$ of all miners was used as a cut-off point.

** FEV 1 : Forced Expiratory Volume in first second; FVC: Forced Vital Capacity; R8Hz: Resistance at $8 \mathrm{~Hz} ; \mathrm{R} 28 \mathrm{~Hz}$ : Resistance at $28 \mathrm{~Hz} ; f_{0}$ : Resonant Frequency; TLC: Total Lung Capacity; DLCO: Diffusion capacity; See methods section for details on CR (chest radiography), HRCT (High Resolution Computed Tomography), and exposure assessment.

*** No statistically significant differences $(p<0.05)$ were detected using either student $t$-test, Mann-Whitney U-test or $\chi^{2}$-test (proportions only). 
Table 12.3 Linear correlations between serum PUP levels and exposure, age, and smoking

\begin{tabular}{|c|c|c|c|}
\hline & group $^{a}$ & group size (n) & correlation coefficient \\
\hline \multirow[t]{3}{*}{ cumulative dust $\left(\mathrm{ghm}^{-3}\right)$} & $\mathrm{HCW}$ & $(76)$ & 0.09 \\
\hline & CWP & $(28)$ & $-0.46^{*}$ \\
\hline & $\mathrm{HCW}$ and CWP & $(104)$ & 0.01 \\
\hline \multirow[t]{3}{*}{ cumulative quartz (mghm ${ }^{-3}$ ) } & $\mathrm{HCW}$ & $(76)$ & 0.09 \\
\hline & CWP & $(28)$ & $-0.43^{*}$ \\
\hline & HCW and CWP & (104) & 0.02 \\
\hline \multirow[t]{3}{*}{ years underground } & $\mathrm{HCW}$ & $(76)$ & 0.05 \\
\hline & CWP & $(28)$ & -0.09 \\
\hline & HCW and CWP & $(104)$ & 0.03 \\
\hline \multirow[t]{3}{*}{ years since first exposure } & $\mathrm{HCW}$ & $(76)$ & -0.21 \\
\hline & CWP & (28) & 0.12 \\
\hline & HCW and CWP & (104) & -0.19 \\
\hline \multirow[t]{4}{*}{ age (years) } & $\mathrm{HCW}$ & (76) & -0.16 \\
\hline & CWP & (28) & 0.04 \\
\hline & NDC & $(29)$ & -0.30 \\
\hline & HCW and CWP & (104) & -0.13 \\
\hline \multirow[t]{4}{*}{ smoking (pack years) } & $\mathrm{HCW}$ & $(76)$ & -0.05 \\
\hline & CWP & (28) & -0.19 \\
\hline & NDC & $(29)$ & -0.08 \\
\hline & HCW and CWP & (104) & -0.07 \\
\hline
\end{tabular}

$a_{\mathrm{HCW}}, \mathrm{CR}=0 / 0 ; \mathrm{CWP}, \mathrm{CR} \geq 0 / 0 ; \mathrm{NDC}$, non dust-exposed control group; ${ }^{7}$ significant at $\mathrm{P}=0.05$

\section{Discussion}

In the present study we evaluated serum PIIIP levels of coal miners in relation to occupational exposure to coal dust, radiographic findings, and lung function in order to evaluate the use of serum PIIIP as an exposre and effect marker [Borm et al. 1992; Borm 1994]. Previously we found that PIIIP levels in serum were increased in active coal miners $(n=73)$ as compared with non-dust exposed controls $(n=19)$, and a downward trend was observed in PIIIP levels versus pneumoconiosis stage as classified by CR [Janssen et al. 1992]. In the current study among retired miners, however, these findings were not confirmed, and no relationship between serum PIIIP and CR [Schins and Borm 1994], or between PIIIP levels and HRCT readings ( $n=46$ ) was found. In addition, serum PIIIP levels were not related to the outcome of flow-volume curves, diffusion capacity or impedance measurements. 

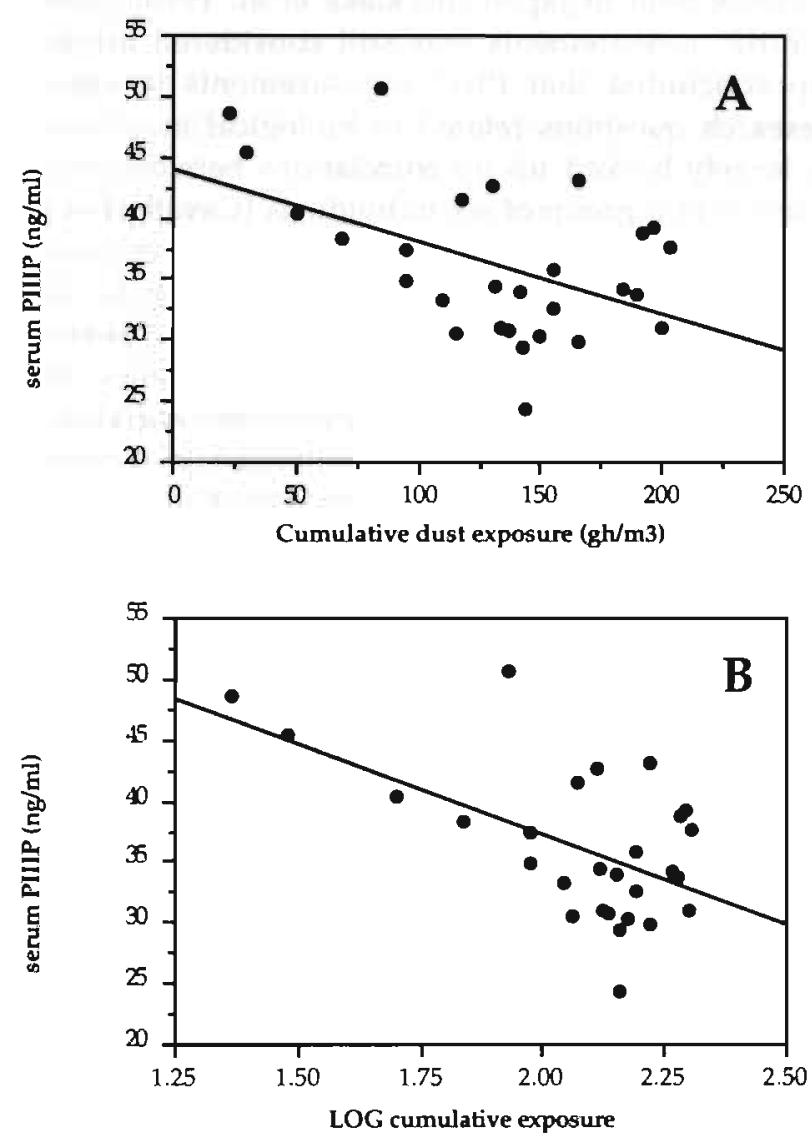

\section{Figure 12.2}

The relation between serum PIIIP and cumulative dust exposure in miners with CWP $(n=28)$, illustrated as linear correlation in panel $A(r=-0.46$, $\mathrm{p}<0.05)$. This correlation improves considerably if exposure is expressed on a logarithmic scale, shown in panel $B(r=-0.55, p<0.005)$.

In contrast to our findings with respect to pulmonary function, Bjermer $e$ t al. reported negative correlations between PIIIP in bronchoalveolar lavage and lung function tests (i.e. VC, $\mathrm{FEV}_{1}, \mathrm{DL}_{\mathrm{CO}}$ ) in sarcoidosis patients [Bjermer et al. 1986]. Similarly, Cavalleri et al. [1991] have reported significantly higher levels of serum PIIIP in asbestos exposed workers with both FVC and $\mathrm{FEV}_{1}$ below the $100 \%$ predicted value compared to workers with values equal to or exceeding this cut-off level. No such relationship between lung function and serum PIIIP levels was observed in our study, using either $100 \%$ or $80 \%$ as a cut-off value for $\mathrm{FEV}_{1}$ and FVC. A reverse analysis, using high and low serum PIIIP as a standard (Table 12.2) failed to reveal any significant difference in lung function, or any other effect, between the two groups. 
Our data are in line with the outcome of a workshop on "health risks from exposure to mineral fibres" recently held in Japan [Becklake et al. 1993], where the diagnositc role of serum PIIIP measurements was still considered limited. However, the working group concluded that PIIIP measurements in serum "could be used to consider research questions related to biological monitoring strategy". This statement was largely backed up by correlations between years exposed to asbestos and PIIIP levels in a group of six individuals [Cavalleri et al. 1988], and more recently in 21 subjects based on calculated asbestos exposure [Cavalleri et al. 1991]. However, our study ( $n=104$ coal miners) did not reveal any clear positive dose-response between serum PIIIP levels and exposure, whether defined as cumulative dust exposure, years underground or years since first exposure. Moreover, a retrospective analysis revealed no significant correlation of PIIIP measured in 1987 and cumulative dust exposure in a subgroup of miners when still active ( $n=51, r=0.13$ ). In addition, years underground, the exposure parameter known for all miners $(n=73)$ in the previous study, was not related to serum PIIIP levels determined at that time (Janssen et al. 1992).

Our data certainly do not suggest that exposure is the only determinant of the observed PIIIP levels, especially in the subgroup of miners with CWP. In this group a significant negative correlation was observed between PIIIP values and ( $\log$ ) cumulative dust exposure. Multiple regression, however, did not reveal other variables (i.e. age, smoking, profusion score, lung function, retirement) involved in the expression of PIIIP in pneumoconiosis. Interestingly, we also observed an inverse relation between cumulative respirable dust exposure and scrum PIIIP in potato sorters [Jorna et al. 1994b] in the absence of radiological evidence for pneumoconiosis. In contrast to the miners in this study, these were all active workers with current exposure to mainly non-crystalline silica. Again no relation between PIIIP and lung function was apparent.

We can only speculate on the underlying reasons for the discrepancies among studies on the relation between PIIIP and respiratory or interstitial effects, but suggest that differences in total cumulative exposure, frequency and intensity of exposure, lung retention time, and mineral characteristics (silica, coal dust, asbestos) are involved. The relation observed between PIIIP and cumulative coal dust exposure, but interestingly not between PIIIP and years underground, suggests a significant effect of intensity and frequency of exposure when compared to duration. Secondly, the time- and clinical course in collagen type III deposition and degradation may also be involved. Both animal and human data have demonstrated that the early increase in type III collagen levels observed in active fibrotic parts of the lung is followed by a significant reduction of type III collagen involvement in favour of other collagen types [Bateman et al. 1981; Laurent and McAnulty 1983; Arden and Adamson 1992]. Therefore, persistent fibrosis might be characterized by lower PIIIP levels, which is supported by 
observations in postmortem biopsies [Seyer et al. 1976; Madri and Furthmayr 1980]. In conclusion, our findings possibly reflect chronic adaptive changes in type III collagen deposition or breakdown, that seem to continue even several years after the cessation of occupational exposure. Whether these may be caused solely by repetitive high peak exposures during occupation [Seaton et al. 1981], or are a consequence merely of the total dust load present in the fibrotic lung remains a question open for further research.

Acknowledgements. The authors thank all coal miners and the Kempense Steenkoolmijnen NV Belgium for their willingness to cooperate in this study; Marc van Sprundel (PhD, MD), Luc Lenaerts (MD), and Luc Mariën (MD) for reading chest radiographs; Dr. Simons of HOECHST AG Germany for performing the PIIIP-Radioimmunoassay. This study is supported by grant no. 7263/03/092 of the European Community for Steel and Coal. 
Whay

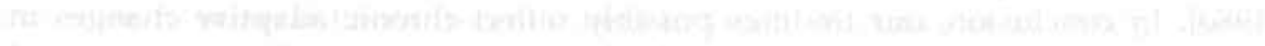

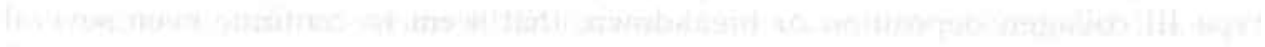

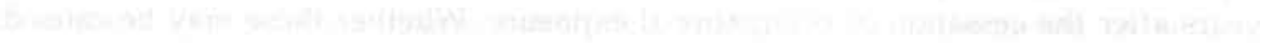

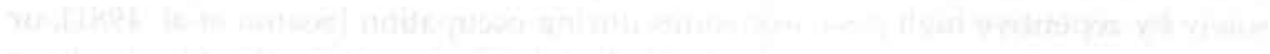

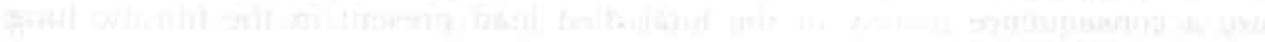

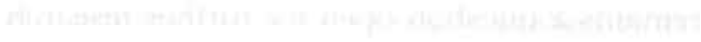




\section{Chapter 13}

\section{SUMMARY AND GENERAL DISCUSSION}

Mineral dust induced lung disorders are among the most widespread and most prevalent occupational lung diseases in the world [Mossman at al., 1990; Cullen et al., 1990; Oxman et al., 1993; Rom, 1992; Meredith and McDonald, 1994]. Most likely as a consequence of the specific toxicokinetic properties of mineral dusts (e.g. durability, particle overload, see chapter 1), disease may even progress after exposure has ended [Jones et al., 1989; Cullen et al., 1990]. In spite of tremendous efforts to reduce exposure, mineral dust induced lung diseases, including pneumoconiosis have not yet ceased to exist, and regularly new cases are being reported both among well established risk groups and less well expected environments [Kauffman et al., 1982; Landrigan et al., 1987; Baris et al., 1988; Cullen et al., 1990; Nemery et al., 1992; Oxman et al., 1993]. Unfortunately, medical treatment of chronic lung disorders such as pulmonary fibrosis is still limited in its efficiency to date [DeRemee, 1994; Goldstein and Fine, 1995; Hunninghake and Kalica, 1995]

As classical occupational epidemiological studies yield less clear cut results due to lower exposure levels (limit values), utilization of biomarkers may be a valuable contribution to epidemiologic research. This also holds for their application to workplace monitoring, medical screening and post-exposure surveillance. Additionally, during the search for these biomarkers, new insights in pathogenic mechanisms may be generated, to identify those at risk or to develop new methods of medical cure and care in those who have become diseased [Schulte et al., 1993; Weill, 1994].

In the present thesis several biological markers of mineral dust induced respiratory disorders were evaluated, by recruitment of coal miners originally involved in several cross-sectional surveys. In other words, molecular epidemiology studies in coal workers linking the "black seam" with "black lungs" have been used to further open the "black box" between mineral dust exposure and resultant chronic lung disorders. This concept is schematically shown in figure 13.1. In the present thesis we focussed on the two mostly appreciated biological mechanisms known to occur in response to mineral dust inhalation, i.e. (1) the generation of reactive oxygen species (ROS) and the related antioxidant defenses, and (2) the involvement of cytokine networks between and among inflammatory cells and structural cells in the lung (see figure 13.1). Furthermore, collagen deposition, recognised as the hallmark of pulmonary fibrosis, was evaluated.

At the time of follow-up all miners had retired from active mining as a result of the closing of the "Kempen" coal mines. Nevertheless in a significant proportion 

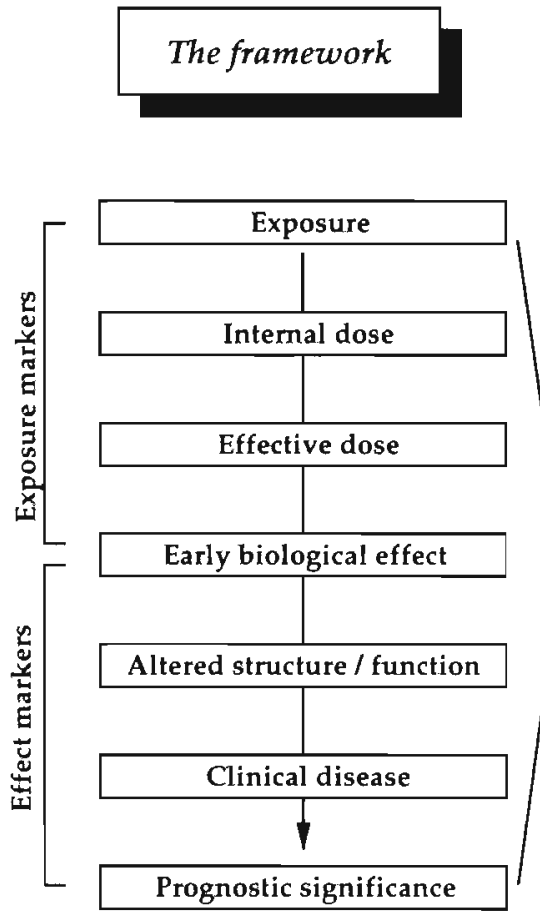

\section{The biological background}

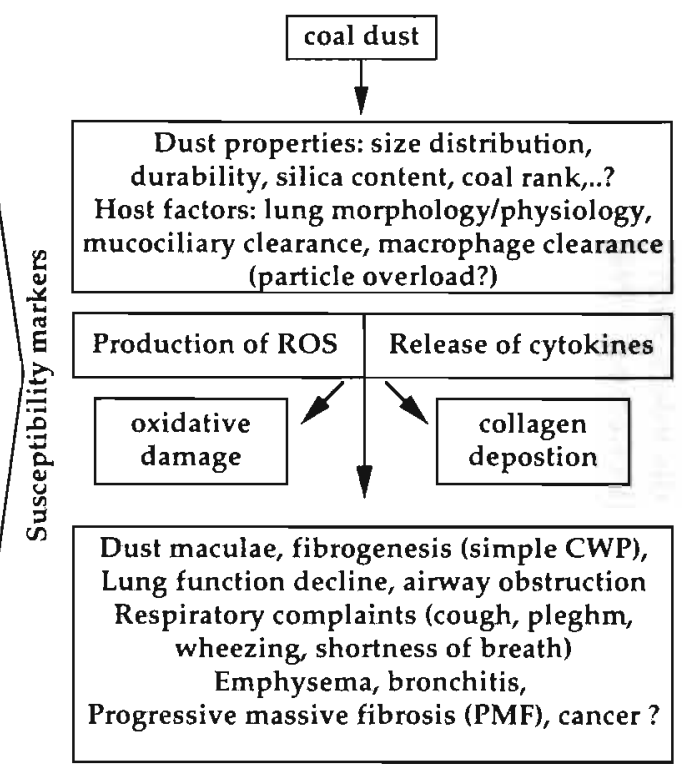

Markers

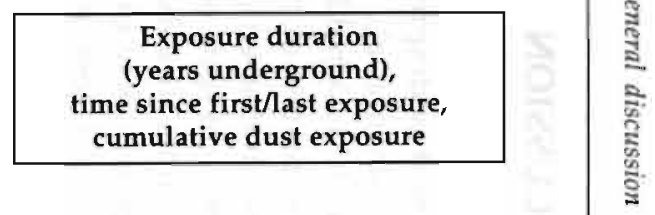

SOD, Cat, GSH,

GST, GPx, VitA VitE, TRAP
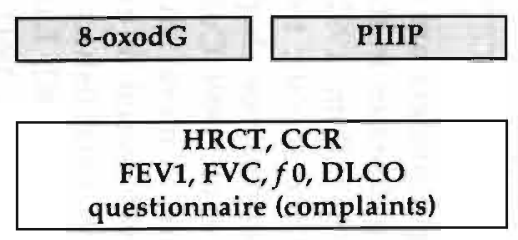

$\triangle \mathrm{CCR}$ $\triangle \mathrm{FEV} 1, \triangle \mathrm{FVC}$

Figure 13.1 Framework of events (left) in mineral dust exposure and resultant lung disease (middle) and biomarkers evaluated (right). The presented framework (described in chapter 1) has been applied to evaluate a set of biological markers that have been forwarded from the biological processes considered to play an important role in mineral dust induced lung disorders (chapters 2 and 3). The markers that have been evaluated in view of this thesis are depicted in the right panel: Peripheral blood parameters (dark box) are related to parameters of mineral dust exposure (upper open box), of lung disorders (middle open box), and of clinical progression of these disorders (lower open box). 
of the miners five-year deterioration of chest radiugraphy determined pneumoconiosis lesions was observed, including identification of some "new" CWP cases (chapter 8 and 11). Estimation of the cumulative exposure to respirable dust by means of a job-exposure matrix approach, showed that although initially the miners had been matched on years of underground employment, actual exposure estimates varied considerably: cumulative dust exposure data had an approximately fourfold variation compared to years underground (chapter 12). Paradoxically, it was this variation which was used to validate markers as "exposure markers", and could explain why few biological events were significantly related to exposure. Interestingly, a good correlation between cumulative dust exposure and conventional chest radiograph- as well as HRCT score was found (chapter 12), and opposite to other exposure parameters, the cumulative dust exposure was clearly related to the presence as well as the progression of CWP (chapter 8). Inclusion of HRCT analysis in a subgroup of miners resulted in a fine-tuning of early stage pneumoconiosis (chapter 12), while validated questionnaires, lung function measurements as well as retrospective analysis of longitudinal lung function decline allowed to relate blood markers to obstructive lung disorders (chapter 3 ). The significance of the biomarkers evaluated in this thesis (see figure 13.1) is discussed below, in line with the structure of the introduction (chapter 3) based on their biological background.

\section{OXIDATIVE DAMAGE AND ANTIOXIDANT STATUS}

The increased ratio of 8-oxodG/dG in lymphocyte DNA of the miners (chapter 6) suggests that oxidative stress responses occur outside the lung. This finding is in line with previous findings from our and other labs [Engelen et al., 1990; NadifPerrin et al., 1995]. They also fit with the results described in chapters 5 and 7, showing that serum and red cell antioxidants are affected in subjects chronically exposed to coal dust. Interestingly, these effects were seen in the absence of acute exposure, since all miners were retired for considerable time at follow-up. Furthermore, these effects are relatively independent of the presence of fibrosis, since increased 8-oxodG levels were already found in those exposed but without CWP. However, from these data one cannot conclude in favour of the suggested direct carcinogenic action of mineral dusts (e.g. silica) as compared to the suggested indirect action via (a) mechanisms occurring in the presence of fibrosis, or via (b) dust acting as carcinogen carriers (e.g. PAH) [Goldsmith et al., 1986; McDonald, 1989]. A possible link between the observed damage in the DNA of lymphocytes and target DNA (e.g. bronchial epithelium) remains to be evaluated, and therefore it is not clear whether this oxidative stress response is related to carcinogenic risk. Nevertheless, our observations are in line with the 
data of Dalal [Dalal et al., 1991] who showed that increased number of stable radicals (determined by ESR) were found in miners compared to controls. The increased number of such stable radicals in lymph nodes of the lung may thus be one explanation for the observed DNA damage in lymphocytes.

Total serum antioxidant capacity, as evaluated by TRAP, was not different between miners with or without CWP, and not related to erythrocyte GSH content (chapter 5). Interestingly however, both TRAP and GSH tended to be higher in those with previous development of CWP (retrospective analysis), while initial GSH, as well as Vitamin E serum levels were lower in subjects with later development of pneumoconiosis (prospective analysis) (chapter 7). It should be realized however, that GSH was determined by different methods in the original study and during follow-up, and Vitamin $E$ is considered to represent only a fraction of the total antioxidant capacity in the serum. In those miners with progression of already existing CWP, also red cell superoxide dismutase activities were higher. Since in vitro and in vivo observations have shown that increased MnSOD expression is an indicator of oxidative stress [Janssen et al., 1994a], one could suggest higher stress in those with progression of CWP as a consequence of increased inflammatory reactions.

When evaluating their applicability as exposure or effect markers it appeared that serum or red cell antioxidants were not related to (cumulative dust) exposure or to severity (chest radiography) of CWP. However, TRAP tended to go down with increased profusion and in those without progression (inactive fibrosis) TRAP was inversely related to cumulative dust exposure (chapter 5). Furthermore red cell antioxidant enzymes (GST, SOD, Catalase) were retrospectively related to lung function decline (chapter 7), and GSH levels were significantly reduced in chronic bronchitis. A particular role may be in store for erythrocyte GST, as reduced activity of this enzyme denominates increased risk for progression of pneumoconiosis, while high activity was associated to increased lung function loss (chapter 7). In conclusion, these data indicate the presence of oxidative stress in subjects chronically exposed to mineral dust (even after mineral dust exposure has ended for considerable time), as well as the significance of these responses in the development or progression of mineral dust induced disorders, including pneumoconiosis and obstructive lung disease. Antioxidant activities in red cells and serum, particularly if they are combined in a multi-marker evaluation, could be implemented in occupational screening as well as in post-occupational surveillance.

\section{CYTOKINE NETWORKS}

Following the observation that ex vivo stimulated release of TNF was increased in active miners [Borm et al., 1988], the crucial involvement of TNF in mineral 
dust induced fibrosis was demonstrated in vivo by Piguet and colleagues in a mouse model of silica induced fibrosis [1990]. In the present study, support for the crucial role of TNF in coal dust induced fibrosis, was obtained in various ways: (1) The repeated cross-sectional analysis (chapter 8) confirmed previous observations [Borm et al., 1988]. (2) The prospective design showed that high monocyte TNF release was clearly related to the risk for progression of CWP (chapter 8). (3) Plasma soluble receptors of TNF (i.e. sTNF-R55 and -R75) were elevated in coal workers compared to non-exposed individuals (chapter 9). The significance of the soluble TNF receptors as susceptibility markers in pneumoconiosis still needs to be evaluated, taking into account a possible confounding effect of medication on plasma sTNF-R55 levels (chapter 9). Interestingly, both erythrocyte SOD activity and monocyte TNF release were risk factors for progression of CWP (chapters 7 and 8). No correlation was found between both parameters at follow-up, although TNF is known as a powerful stimulus of mRNA expression of MnSOD in various target cells [Wong and Goeddel., 1988]. Monocyte release of TNF was not related to any parameter of exposure, both in the original [Borm et al., 1988] and the follow-up study (chapter 8). Significant correlations between TNF release measured in the original study and at follow-up, were found in response to coal dust or $3 \mathrm{ng} / \mathrm{ml}$ LPS, and -more specifically- in individuals without progression of CWP only. The latter observation indicates that during to course of disease the ability of monocytes to release TNF upon stimulation may change (chapter 8 and chapter 10). In addition, compared to the original study in active miners, spontaneous release of TNF from monocytes was significantly reduced in retired miners compared to non-exposed controls (chapter 8). These observations suggest that baseline release of TNF is determined by active or current exposure, while stimulated release of TNF is related to past (cumulative) exposure, or to the presence of accumulated mineral dust in the lung. This is in contradiction with TGF data where both spontaneous and dust stimulated release (measured in the same monocyte supernatants) was higher in retired miners compared to the non-exposed individuals (chapter 10). The relation between monocyte release of TNF, TGF and IL- 6 was described in chapter 10. Generally, IL-6 release from monocytes was found to be comparable to TNF release, except in silica incubations. This might be closely related to differences in the hazards of silica versus coal dust [Gosset et al., 1991], as well as differences in silicosis versus CWP pathology.

Most interestingly, stimulated release of TNF and TGF were positively correlated in non-exposed individuals, but inversely related to each other in subjects with CWP. TGF has recently been forwarded as an important regulator of GSH, [Arsalane et al., 1995], and decreased GSH status is considered as an important risk factor of fibrosis [Cantin et al., 1990]. In line with these observations, we found a negative correlation between monocyte TGF release and erythrocyte GSH (chapter 10). An important question still to be answered in 
view of these observations is the concept of priming. Further research is needed to explain why different in vitro responses of peripheral blood monocytes reflect differences in the lungs of non-exposed, exposed, formerly exposed, and diseased subjects.

Gathering the evidence from in vivo animal studies and the epidemiological follow-up, the crucial role of TNF in mineral dust induced fibrosis is now established [Piguet et al., 1990], and the priming of monocytes in retired miners as well as increased TNF receptor levels suggest that systemic effects may occur in response to mineral dust exposure. Important to health surveillance, the risk for progression of CWP is related to individual monocyte TNF release. Whether or not these phenotype variations in TNF release are related to a genetic predisposition, e.g. based on polymorphisms of TNF promotor regions [Pociot et al., 1993] needs further study. TGF appears another potent candidate crucially involved in (the progression of) CWP (to PMF) [Vanhée et al., 1995]. Prospective follow-up of the present cohort, as well as external validation in other cohorts of miners will elucidate the predictive power of combined measurement of TNF and TGF and/or TNF and its receptors. These studies are currently in progress, using a simplified screening assay using whole blood incubations [Schins et al., 1996].

\section{COLLAGEN}

In chapter 12 we investigated whether serum PIIIP levels can be considered as a biomarker of chronic coal dust exposure or of coal dust induced respiratory disorders, including fibrosis and obstructive lung disease. PIIIP levels were neither related to lung function parameters (spirometry, impedance measurement, diffusion capacity), nor to chest radiography lesions as visualized by conventional chest radiography or high resolution computed tomography. Furthermore PIIIP levels were not related to cumulative dust exposure or to any other parameter of exposure, although PIIIP levels tended to decrease with increased cumulative exposure in miners with CWP (chapter 12). These negative findings are in sharp contrast to positive studies related to mineral dust exposure and/or lung function in subjects exposed to asbestos [Cavalleri et al., 1991], as well as in sarcoidosis patients [Bjermer et al., 1986]. Longitudinal analysis of PIUP levels in active coal workers in relation to radiological progression of CWP (described in chapter 11) did not forward PIIIP as a susceptibility marker to development or progression of pneumoconiosis, opposite to observations in sarcoidosis patients [Pohl et al., 1992]. Unfortunately, we are not able to discriminate between retirement, normal intra-individual variation, or drift in the assay to explain for the significant differences in individual PIIIP release at the two time points measured. However, the lack of "abnormal" high PIIIP 
values at follow-up (i.e. values above $2 \times$ S.D. of non-exposed individuals) is in favour of the first option, i.e. a reduction of serum PIIIP in response to cessation of mineral dust exposure. However, a (positive) correlation between PIIIP levels and cumulative exposure which would have supported this hypothesis, was not found at the two time points measured (chapters 11 and 12). All in common these data are not in favour of PIIIP as useful biomarker in mineral dust exposure. Nevertheless, it may seem relevant to determine the predictive values of measurement of combined collagens such as PIIIP in combination with type VI related collagen, since this is considered to allow for parallel assessment of both collagen synthesis and collagen degradation [Schuppan et al., 1994]. In addition, further studies (e.g. in situ analysis of collagens) are needed to determine the exact kinetics of type III collagen formation as well as other relevant collagens during the development of mineral dust induced fibrosis.

The use of "marker" approaches in occupational lung disease, and more specifically in relation to coal dust exposure is not new. For instance, nutritional status of miners has been considered as a risk factor explaining respiratory disorders in coal miners [Swartz et al., 1978]. In fact, lung function measurements themselves can be considered as important markers and have been used in routine screening of miners for many decades [Attfield and Wagner, 1482]. Interestingly, in many studies among coal miners a consistent relation between FEV1-decline and cumulative dust exposure has been reported in a range of 0.4 to $0.8 \mathrm{~mL} \cdot \mathrm{gh}^{-1} \cdot \mathrm{m}^{-3}$ [Wouters et al., 1994]. Radiological stage of simple (WP, as well as its progression rate, have been regarded as indicative "markers" for the development of PMF [Gautrin et al., 1994]. Nowadays, HRCT analysis is applied to allow for a more sensitive detection of CWP, i.e. closer to the time of exposure in the exposure disease continuum (see chapter 2). Apart from differences in intrinsic biological responses, susceptibility differences in mineral dust induced disorders may also be related to internal or effective dose and determined by inter-individual variation in deposition of particles in the lung [Muir, 1991] or mucocilliary clearance [Bohning and Lippman, 1992] (see figure 13.1). In fact, observations in a sheep model of asbestosis, forwarded particle clearance as a susceptibility marker [Bégin et al., 1989; Borm, 1994]. Elucidation of the crucial role of elastases in emphysema and the large inter-individual variation of neutrophil elastases, has led to a denomination of this mechanism to explain for risk for lung emphysema [Karlinski et al., 1978; Hubbard et al. ,1990].

A number of biological events or parameters occurring during coal dust exposure could be evaluated in this thesis (see figure 13.1), allowed validation of a set of biomarkers of mineral dust induced lung disorders, rejection of some 
others, as well as generation of some new candidate markers. In Table 13.1 the most important findings are briefly summarized.

Table 13.1 Biomarkers evaluated in this thesis in relation to their application in molecular epidemiology of coal dust induced lung disorders

\begin{tabular}{ll} 
Marker & Event \\
\hline TNF & progression of CWP, lung function decline \# \\
TGF & chronic dust exposure \\
IL-6 & presence of CWP \\
Vitamin A, E & obstructive lung disease \\
TRAP & progression of CWP (retrospective analysis) \\
SOD & progression of CWP, lung function decline \\
Catalase & progression of CWP, lung function decline \\
GSH/GSSG & Chronic bronchitis, progression of CWP \\
Gpx & Lung function decline, relation with GSH/GSSG, GST \\
GST & discrimination between interstitial and respiratory disease \\
8-oxodG/dG & Oxidative stress due to (former) exposure \\
PIIIP & Active fibrosis \\
\hline
\end{tabular}

\# Described in Joma et al., 1994

It should be emphasized that the decision to recruit subjects involved in crosssectional surveys for follow-up has allowed to test the validity of several of these biomarkers. The strength of this study (the prospective analysis) also brings along its weaknesses. Inherent to a longitudinal survey, markers initially measured, or the method by which they are analysed may be obsolete at the time of follow-up [Rothman et al., 1993]. Furthermore, the design of the original studies was casecontrol, and to investigate the involvement of cytokines, antioxidants and PIIIP in pneumoconiosis, miners were matched on age and exposure [Borm et al., 1990; Janssen et al., 1992a]. Statistical limitations are caused by the size of the population studied and the period of follow-up, in view of the incidence and prevalence of CWP. Indeed, more preferable methods to validate biomarkers could be applied, such as larger scaled prospective cohort studies (based on detailed power calculations), or multi-center studies [Schulte and Rothman, 1993]. Despite these limitations, the present approach allowed a fast validation of candidate biomarkers measured at earlier time. Furthermore, new candidates have been forwarded and related to the (validated) markers, at the same time leading to increased understanding of underlying biological mechanisms of coal dust induced lung disorders.

In relation to the implementation of present markers (see Table 13.1) the question rises, as to how far present markers can be extrapolated from "coal" workers to populations exposed to other mineral dusts (e.g silica or asbestos). Based on the common outcome of disease (chapter 1 and chapter 2) and the 152 
similarity in their underlying biological mechanisms (chapter 3), at least for several markers this extrapolation may hold true: Considering the key role of the macrophage in mineral dust inhalation, as well as the suggested macrophagefibroblast-pathway in its fibrogenic response [Heppleston and Styles, 1967], monocyte release of TNF most likely is a valid susceptibility marker in any mineral dust induced fibrogenicity. Evidently its exact predictive value needs to be assessed in other cohorts. Furthermore, the concomitant measurement of monocyte release of TGF and/or plasma soluble TNF receptors may have additional value, although they also need to be evaluated in a prospective survey. Serum and red cell antioxidants, particularly erythrocyte SOD activity and GSH concentration, have been validated by such a prospective design (chapter 7). Supported by several in vitro and in vivo observations (chapter 3), also the antioxidant route (see figure 13.1) most likely is a common mechanism in response to various mineral dusts and related disease, and indicate general application of red cell and serum antioxidant status as markers in fibrogenesis. Also in non-dust induced fibrogenic lung diseases, both cytokines (e.g. TNF, TGF) and ROS are considered to play important roles, and cytokine-antagonist (e.g. TNF antibodies) as well as antioxidants (e.g. oral $\mathrm{N}$-acetylcysteine) are considered as promising therapies [Goldstein and Fine, 1995; Hunninghake and Kalica, 1995].

In sharp contrast, serum PIIIP was rejected as marker of coal dust exposure, opposite to previous findings in relation to asbestos exposure [Cavalleri et al., 1988; 1991]. Furthermore, this marker was demonstrated to have predictive value in determining disease (progression) in sarcoidosis [Pohl et al., 1992], however not in CWP (chapter 11). Thus, unlike several cytokines and antioxidant factors, serum PIIIP cannot be considered as a general marker of mineral dust exposure, or on the other hand of fibrotic lung disease.

The relations of some of the markers to nonpneumoconiotic effects, and their independence with pneumoconiotic effects in our studies, suggest a possible application beyond fibrogenic lung disease. An increase of TNF in obstructive lung disease in coal workers has been described previously [Jorna et al., 1994], and the relation between serum and red cell antioxidants and lung function decline, as well as between GSH and chronic bronchitis, was shown in this thesis. These findings may be confirmed, if the present cross-sectional and retrospective analyses of antioxidants and cytokines in relation to lung function decline and chronic bronchitis hold true in a prospective design i.e. by continuation of the follow-up of the present study group or in other surveys. Since lung function decline may be considered as a relatively non-specific response to a variety of mineral dusts (see chapters 1 an 2) as well as other exposures, the present markers are likely to have significant meaning in other occupational settings. To evaluate the use in the absence of occupational exposure, evidently further study in non-exposed individuals is needed. The relevance of oxidative DNA damage 
in lymphocytes in relation to various lung disorders seems closely related to antioxidant parameters evaluated. This parameter may therefore be considered as valid marker of oxidative stress in response to chronic inhalation of mineral dusts. However, in relation to the cancer risk associated with (some) mineral dusts, the practical use of this marker is severely limited by the incidence and prevalence of these disorders, as well as their long latency.

The hallmarks of "molecular epidemiology" -although some molecular biologists and epidemiologists continue to debate on the rudeness of this formulation- are inherent to curiosity of mankind since the dawn of history: First, there is our need to explain why and, more important, how we are dealing with diseases. In the population studied in this thesis, the causal agent is known (see chapter 2). In vitro and in vivo studies (chapter 3 ) have lead to the clarification of important biological mechanisms playing a role in the development or progression of coal dust induced lung disorders. The studies described in this thesis further increased our understanding in these diseases.

Elucidation of the cause of a disease, almost directly leads to a second question: "why are we not all equally susceptible?" This question is obvious in the well known symbolism of two colliers who worked shoulder to shoulder for many decades at the same pit, the one in perfect shape, the other one suffering from severe shortness of breath. To explain (at least in part) for these aspects, in the studies described in the present thesis, several markers of mineral dust induced lung disorders were generated. The next step is to use these markers. This inevitably needs extrapolation of sophisticated, expensive and time consuming intra-laboratory methods, into simplified assays to implement these markers in large populations in workplace settings were laboratory conditions are limited. As stated before, these studies are currently in progress. The use of these markers has also ethical consequences [Schulte, 1991], since application of validated biomarkers, may "discriminate" individuals beyond the biological framework of (occupational) exposure and resultant disease. Both ethical and legal aspects of the use of biomarkers will need considerable attention in the future.

It is recommended to further evaluate presented markers -or combination of markers- in relation to specific research questions, such as their predictive value in coal workers at the start of their employment, in PMF patients, as well as in relation to other (mineral) dust exposures including workers employed in ceramic industry, in grinding or sandblasting, surface miners, construction workers etc. The work presented in this thesis may serve as a guide to implement both the presented framework of biomarkers (see figure 13.1) of mineral dust related disease and the generated (candidate) markers (see Table 13.1) in such surveys. 
References 



\section{References}

Abraham JL. Environmental pathology of the lung. In: Rom WN, Ed. Environmental and Occupational Medicine, 2nd edition. Little, Brown and Company, Boston, 1992, pp. 227-53.

Adachi S, Yoshida S, Kawamura K, Takahashi M, Uchida H, Odagiri $Y$, Takemoto $\mathrm{K}$. Inductions of oxidative DNA damage and mesothelioma by crocidolite, with special reference to the presence of iron inside and outside of asbestos fiber. Carcinogenesis 1994;15:753-8.

Aderka D, Engelmann H, Hornik V, Skorick Y, Levo Y, Wallach D, Kushtai G. Increased levels of soluble receptors for tumor necrosis factor in cancer patients. Cancer Res 1991;51:5602-7.

Aebi H. Catalase in vitro. In: Beutler E, Ed. Methods of enzymology, vol 105, New York, Academic Press, 1984, pp. 121-6.

Aksamit TR, Monick MM, Hunninghake GW. Protein kinase C modulates the amounts of IL-1 receptor mRNA in human lung fibroblasts. J Immunol 1993;151:284-90.

Altekruse EB, Chaudhary BA, Pearson MG, Morgan WKC. Kaolin dust concentrations and pneumoconiosis at a kaolin mine. Thorax 1984; 39:436-41.

Amento EP, Bhan AK, McCullagh KG, Krane SM. Influences of gamma interferon on synovial fibroblast-like cells. Induction and inhibition of collagen synthesis. J Clin Invest 1985;76:837-48.

American Thoracic Society/ATS. Standards for the diagnosis and care of patients with chronic obstructive pulmonary disease (COPD) and asthma. Am Rev Respir Dis 1987;136:225-44.

Ames BN, Cathcart R, Schwiers E, Hochstein P. Uric acid provides an antioxidant defense in humans against oxidant- and radical-caused aging and cancer: a hypothesis. Proc Natl Acad Sci USA $1981 ; 78: 6858-62$.

Anderson ME. Determination of glutathione and glutathione disulfide in biological samples. Meth Enzymol 1985;113:548-55.

Antoniades HN. Human platelet-derived growth factor (PDGF): purification of PDGF-I and PDGF-II and separation of their reduced subunits. Proc Natl Acad Sci USA 1981;78:7314-7.

Anttinen H, Terho EO, Myllylä, Savolainen E-R. Two serum markers of collagen biosynthesis as possible indicators of irreversible pulmonary impairment in Farmer's Lung. Am Rev Respir Dis 1986;133:88-93.

Arden MG, Adamson IYR. Collagen synthesis and degradation during the development of asbestosinduced pulmonary fibrosis. Exp Lung Res 1992;18:9-20

Arsalane K, Muanza T, Bégin R, Cantin A. Transforming growth factor- $\beta$ enhances A549 cell susceptibility to hydrogen peroxide mediated injury. Am J Respir Crit Care Med 1995;151:A64.

Aruoma OI, Halliwell B, Dizdaroglu N. Iron ion-dependent modification of bases in DNA by the superoxide radical-generating system hypoxanthine/xanthine oxidase. J Biol Chem $1989 ; 264: 13024-8$.

Assoian RK, Fleurdelys BE, Stevenson HC, Miller PJ, Madtes DK, Raines EW, Ross R, Sporn MB. Expression amd secretion of type beta transforming growth factor by activated human macrophages. Proc Natl Acad Sci USA 1987;84:6020-4.

Attfield MD. Longitudinal decline in FEV1 in United States coal miners. Thorax 1985;40:132-7.

Attfield MD, Hodous TK. Pulmonary function of US coal miners related to dust exposure estimates. Am Rev Respir Dis 1992;145:605-9.

Attfield M, Wagner G. Respiratory disease in coal miners. In: Rom WN, Ed. Envirunmental and Occupational Medicine, 2nd edition. Little, Brown and Company, Boston, 1992, pp. 325-44.

Babior BM, Kipnes RS, Curnutte JT. Biological defense mechanisms. The production by leukocytes of superoxide, a potential bactericidal agent. J Clin Invest 1973;52:741-4.

Baris YI, Bilir N, Artvinli M, Sahin AA, Kalyoncu F, Sebastien P. An epidemiological study in an Anatolian village environmentally exposed to tremolite asbestos. $\mathrm{Br} \mathrm{J}$ Ind Med 1988;45:838-40.

Barnes PJ. Reactive oxygen species and airway inflammation. Free Rad Biol Med 1990; 9:235-43.

Bateman ED, Turner-Warwick M, Adelmann-Grill BC. Immunohistochemical study of collagen types in human foetal lung and fibrotic lung disease. Thorax 1981;36:645-53. 
Battegay EJ, Raines EW, Seifert RA, Bowen-Pope DF, Ross R. TGF-beta induces bimodal proliferation of connective tissue cells via complex control of an autocrine PDGF loop. Cell $1990 ; 63: 515-24$.

Becklake M. Occupational exposures and chronic airways disease. In: Rom WN, Ed. Environmental and Occupational Medicine, 2nd edition. Little, Brown and Company, Boston, 1992, pp. 453-63.

Becklake M, Bignon J, Brochard P, Chiappino G, Cavalleri A, Ward Gardner A., et al. Biologic indicators and their clinical significance in persons exposed to mineral fibres: report of a workshop held in Japan, 24-25 November 1991. Br J Ind Med 1993:50:412-7.

Beckman JS, Beckman TW, Chen J, Marshall PA, Freeman BA. Apparent hydroxyl radical production by peroxinitrite: implications for endothelial injury from nitric oxide and superoxide. Proc Natl Acad Sci USA 1990;87:1620-4.

Bégin R, Masse S, Sébastien P. Alveolar dust clearance capacity as determinant of individual susceptibility to asbestosis: new experimental observations. Ann Occup Hyg 1989;33:279-82.

Bégin R, Ostiguy G, Filion R, Colman N, Bertrand P. Computed tomography in the early detection of asbestosis. $\mathrm{Br}$ J Ind Med 1993;50: 689-98.

Bégin R, Ostiguy G, Filion R, Colman N. Computed tomography scan in the early detection of silicosis. Am Rev Respir Dis 1991;144:697-705.

Bemelmans MHA, Gouma DJ, Buurman WA. Influence of nephrectomy on tumor necrosis factor clearance in a murine model. J Immmunol 1993; 150: 2007-17.

Beutler B, Cerami A. The endogenous mediator of endotoxic shock. Clin Res 1987;35:192-7.

Beutler B, Greenwald D, Hulmes JN, Chang M, Phan YCE, Mathison J, Ulevich R, Cerami A. Identity of tumor necrosis factor and the macrophage-secreted cachectin. Nature 1985;316:552-4.

Beutler B, Grau GE. Tumor necrosis factor in the pathogenesis of infectious diseases. Crit Care Med 1993;21:S423-35.

Bienkowski RS. Interstitial collagens. In: Crystal RG, West JB, Barnes PJ, Cherniack NS, Weibel ER, Eds. The lung. Scientific Foundations. Raven Press, New York, 1991, pp. 381-8.

Bienkowski RS, Gotkin MG. Control of collagen deposition in mammalian lung. Proc Soc Exp Biol Med 1995;209:118-40.

Bissonette E, Rola-Pleszczynski M. Pulmonary inflammation and fibrosis in a murine model of asbestosis and silicosis. Possible role of tumor necrosis factor. Inflammation 1989;13:329-39.

Bitterman PB, Adelberg S, Crystal RG. Mechansisms of pulmonary fibrosis. Spontaneous release of the alveolar macrophage-derived growth factor in the interstitial lung disorders. J Clin Invest 1983;72:1801-13.

Bitterman PB, Rennard SI, Hunninghake GW, Crystal RG. Human alveolar macrophage growth factor for fibroblasts. Regulation and partial characterization. J Clin Invest 1982;70:806-22.

Bitterman PB, Wewers MD, Rennard S1, Adelberg S, Crystal RG. Modulation of alveolar macrophage-driven fibroblast proliferation by alternative macrophage mediators. J Clin Invest 1986;77:700-8.

Bjermer $L$, Thunell M, Hällgren R. Procollagen III peptide in bronchoalveolar lavage fluid. Lab Invest 1986;55:654-6.

Bohning DE, Lippmann M. Particle deposition and pulmonary defense mechanisms. In: Rom WN, Ed. Environmental and Occupational Medicine, 2nd edition. Little, Brown and Company, Boston, 1992, pp. 171-82.

Bolton RE, Vincent HJ, Jones AD, Addison J, Beckett ST. An overload hypothesis for pulmonary clearance of UICC amosite fibers inhaled by rats. Br J Ind Med 1983;40:264-72.

Bonner JC, Brody AR. Cytokine-binding proteins in the lung. Am J Physiol 1995; 268:L869-78.

Bonner JC, Badgett A, Lindroos PM, Osornio-Vargas AR. Transforming growth factor beta 1 downregulates the platelet-derived growth factor alpha receptor subtype on human lung fibroblasts in vitro. Am J Respir Cell Mol Biol 1995;13:496-505.

Borm PJA, Bast A, Wouters EFM, Slangeri JJ, Swaen GMH, De Boorder Tj. Red blood cell antioxidant parameters in healthy elderly versus silicosis patients. Free Rad Res Comm 1986; 3: $117-27$. 
Borm PJA, Palmen N, Engelen JJM, Buurman WA. Spontaneous and stimulated release of tumor necrosis factor (TNF)-alpha from blood monocytes of miners with coal. workers' pneumoconiosis. Am Rev Respir Dis 1988;138:1589-94.

Borm P, Meijers J, Swaen G. Molecular epidemiology of coal worker's pneumoconiosis: Application to risk assessment of oxidants and monokine generation by mineral dusts. Exp Lung Res 1990;16:57-71.

Borm PJA, Schins R, Janssen YMW, Lenaerts L. Molecular basis for differences in susceptibility to coal workers' pneumoconiosis. Toxicol Letters 1992;64/65:767-72.

Borm PJA, Schins PFR. Lymphocyte subsets in subjects exposed to asbestos: changes in circulating natural killer cells. Br J Ind Med 1993;50:575.

Borm PJA. Biological markers and occupational lung disease. Exp Lung Res 1994;20:457-70.

Bowcock AM, Kidd JR, Lathrop GM,Daneshvar L, May LT, Ray A, Sehgal PB, Kidd KK, CavalliSforza LL. The human "interferon-beta $2 /$ hepatocyte stimulating factor/interleukin- 6 " gene: DNA polymorphism studies and localization to chrmosome 7p21. Genomics 1988;3:8-16.

Bowden DH. Macrophages, dust, and pulmonary diseases. Exp Lung Res 1987;12:89-107.

Bowden DH. Human exposure and effects: Synthesis. In: Mohr U, Dungworth DL, Mauderly JL, Oberdörster G, Eds. Toxic and Carcinogenic Effects of Solid Particles in the Respiratory Tract. ILSI Press, Washington, 1994, pp. 329-31.

Britton JR, Pavord ID, Richards KA, Knox AJ, Wisniewski AF, Lewis SA, Tattersfield AE, Weiss ST. Dietary antioxidant vitamin intake and lung function in the general population. Am J Respir Crit Care Med 1995;151:1292-3.

Brockhaus M, Schoenfeld H-J, Schlaeger E-J, hunziker W, Lesslauer W, Loetscher H. Identification of two types of tumor necrosis factor receptors on human cell lines by monoclonal antibodies. Proc Natl Acad Sci USA 1990; 87: 3127-31.

Brody AR, Bitterman PB, Adler KB, Rannels DE, Thet LA, Rom WN, Rennard SI. The lung matrix and inflammation: Part II. Biochemical and molecular mechanism of fibrogenesis: implications for environmental lung disease. Am Rev Respir Dis 1988;138:1056-7.

Brody AR. Asbestos-induced lung disease. Env Health Perspect 1993;100:21-30.

Brody AR, Roe MW, Evans JN, Davis GS. Deposition and translocation of inhaled silica in rats. Quantification of particle distribution, macrophage participation, and function. Lab Invest 1982;47:533-42.

Broekelman TJ, Limper AH, Colby TV, McDonald JA. Transforming growth factor- $\beta 1$ is present at sites of extracellular matrix gene expression in human pulmonary fibrosis. Proc Natl Acad Sci USA 1991;88:6642-6.

Brown JM,Beehler CJ, Berger EM, Grosso MA, Whitman GJ, Terada LS, Leff JA, Harken AH, Rupine JE. Albumin decreases hydrogen peroxide and reperfusion injury in isoluted rat hearts. Inflammation 1989a;13:583-9.

Brown GM, Donaldson K, Brown DM. Bronchoalveolar leukocyte response in experimental silicosis: Modulation by a soluble aluminum compound. Toxicol Appl Pharmacol 1989b;101:95-105.

Burton GW, Ingold KU. Autooxidation of biological molecules. I. The antioxidant activity of vitamin $E$ and related chain-breaking phenolic antioxidants in vitro. J Am Chem Soc 1981;103:6427-77.

Cantin A, Crystal RG. Oxidants, antioxidants and the pathogenesis of emphysema. Eur J Respir Dis 1985;66:7-17.

Cantin AM, Larivee P, Bégin R. Extracellular glutathione supresses human lung fibroblast proliferation. Am J Respir Cell Mol Biol 1990; 3:79-85.

Cantin AM, Boileau R, Bégin R. Increased procollagen III aminoterminal peptide-related antigens and fibroblast growth signals in the lungs of patients with idiopathic pulmonary fibrosis. Am Rev Respir Dis 1988;137:572-8.

Cantin A, North SL, Fells GA, Hubbard RC, Crystal G. Normal alveolar epithelial lining fluid contains high levels of glutathione. J Appl Physiol 1987a;63:152-7.

Cantin A, North SL, Hubbard RC, Crystal RG. Oxidant-mediated epithelial cell injury in idiopathic pulmonary fibrosis. J Clin Invest 1987b;79:1665-73. 
Cantin A, North SL, Crystal RG. Glutathione is present in normal epithelial lining fluid in sufficient concentration to provide antioxidant protection in lung parenchymal cells. Am Rev Respir Dis 1985;131:A372.

Carswell EA, Old LJ, Green S, Fiore N, Williamson B. An endotoxin-induced serum factor that causes necrosis of tumors. Proc Natl Acad Sci USA 1975;72:3666-70.

Cavalleri A, Gobba F, Bacchella L, Luberto F, Ziccardi A. Serum type III procollagen peptide in asbestos workers: an early indicator of pulmonary fibrosis. Br J Ind Med 1988;45:818-23.

Cavalleri A, Gobba F, Bacchella L, Ferrari D. Evaluation of serum aminoterminal propeptide of type III procollagen as an early marker of the active fibrotic process in asbestos-exposed workers. Scand J Work Environ Health 1991;17:139-44.

Cerami A, Ideka Y, Le Trang N, Hotez PJ, Beutler B. Weight loss associated with an endotoxininduced mediator from peritoneal macrophages: the role of cachectin (tumor necrosis factor). Immunol Lett 1985;11:173-7.

Chance B, Sies H, Boveris A. Hydroperoxide metabolism in mammalian organs. Physiol Rev 1979:59:527-605.

Chaudri G, Clark IA. reactive oxygen species facilitate the in vitro and in vivo lipopolysaccharide-induced release of tumor necrosis factor. J Immunol 1989;143:1290-4.

Churg A, Wright JL, Wiggs N, Pare PD, Lazar N. Small airways disease and mineral dust exposure. Am Rev Respir Dis 1985;131:139-43.

Chvapli M, Peng YM. Oxygen and lung fibrosis. Arch Environ Health 1975;30:528-32.

Clark JG, Madtes MK, Raghu G. Effects of platelet-derived growth factor isoforms on human lung fibroblast proliferation and procollagen gene expression. Exp Lung Res 1993;19:327-44.

Clarke SW, Pavia D. Mucociliairy clearance. In: Crystal RG, West JB, Barnes PJ, Cherniack NS, Weibel ER, Eds. The lung. Scientific Foundations. Raven Press, New York, 1991, pp. 1845-59.

Cochrane AL. Relation between radiographic categories of pneumoconiosis and expectation of life. $\mathrm{Br} J$ Ind Med 1973;2:532-4.

Collis EL, Gilchrist JC. Effects of dust upon coal trimmers. J Ind Hyg 1928;10:101-10.

Cooke WE. Pulmonary asbestosis. Br Med J 1927;2:1024-5.

Cotes JE, Steel J, Leathart GL. Work related lung disorders. Blackwell Sci Publ, Oxford, 1987, pp. 436.

Crapo JD, Oury T, Rabouille C, Slot JW, Chang LJ. Copper-zinc dismutase is primarily a cytosolic protein in human cells. Proc Natl Acad Sci USA 1992;89:10405-9.

Crystal RG, Ferrans VJ, Basset F. Biologic basis of pulmonary fibrosis. In: Crystal RG, West JB, Barnes PJ, Cherniack NS, Weibel ER, Eds. The lung. Scientific Foundations. Raven Press, New York, 1991, pp. 2031-46.

Crystal RG. Alveolar macrophages. In: Crystal RG, West JB, Barnes PJ, Cherniack NS, Weibel ER, Eds. The lung. Scientific Foundations. Raven Press, New York, 1991, pp. 527-538.

Cuesta-Sanz D, Santa-Cruz MC. Simultaneous measurement of retinol and $\alpha$-tocopherol in serum by high-performance liquid chromatography with ultraviolet detection. J. Chromatogr 1986;380:140-4.

Cullen MR, Cherniak MG, Rosenstock L. Medical progress. Occupational Medicine. N Engl J Med 1990;322:594-601.

Cummins SL. The pneumoconioses in South Wales. J Hyg 1936;36:547-8.

Dalal NS, Shi $X$, Vallyathan V. Potential role of silicon-oxygen radicals in acute lung-toxicity. In: Mossman BT, Bégin RO, Eds. Effects of mineral dusts on cells. Springer Verlag, Berlin, 1989, pp. 265-72.

Dalal NS, Jafari B, Petersen M, Green FH, Vallyathan V. Presence of stable coal radicals in autopsied coal miners' lungs and its possible correlation to coal worker's pneumoconiosis. Arch Environ Health 1991;46:366-72.

Dalal NS, Newman J, Pack D, Leonard S, Vallyathan V. Hydroxyl radical generation by coal mine dust: possible implication to coal worker's pneumoconiosis (CWP). Free Radic Biol Med 1995; 18:11-20. 
Daniel LN, Mao Y, Saffiotti U. Oxidative DNA damage by crystalline silica. Free Rad Biol Med 1993;14:463-72.

Davis GS, Calhoun WJ. Occupational and environmental causes of interstitial lung disease. In: Schwarz MI, King TE, Eds. Interstitial lung disease. B.C. Dekker Inc. Toronto, 1988, pp. 63-109.

Davies R, Erdogdu G. Secretion of fibronectin by mineral dust-derived alveolar macrophages and activated peritoneal macrophages. Exp Lung Res 1989;15:285-97.

Dayer JM, Beutler B, Cerami A. Cachectin/tumor necrosis factor stimulates collagenase and prostaglandin E2 production by human synovial cells and dermal fibroblasts. J Exp Med $1985 ; 162: 2163-8$.

Dayer JM, de Rochemonteix B, Burrus B, Demczuk S, Dinarello CA. Human recombinant interleukin1 stimulates collagenase and prostaglandin E2 production by human synovial cells. J Clin Invest 1986;77:645-8.

Dean DC, Newby RF, Bourgeois S. Regulation of fibronectin biosynthesis by dexamethasone, transforming growth factor beta, and cAMP in human cell lines. J Cell Biol 1988;106:2159-70.

Debets JMH, van der Linden CJ, Spronken IEM, Buurman WA. T-cells mediated production of TNFalpha by monocytes. Scand J Immunol 1988;27:601-9.

Dentener MA, Bazil V, Von Asmuth EJU, Ceska M, Buurman WA. Involvement of CD14 in lipopolysaccharide-induced tumor necrosis factor- $\alpha$, IL- 6 and IL- 8 release by human monocytes and alveolar macrophages. J Immunol 1993;150:2885-91.

DeRemee. New approaches to the treatment of pulmonary fibrosis. Eur Respir J 1994;7:427-8.

DECOS. Brief evaluation of carcinogenic substances. Silica Update 1995/1996. Dutch Expert Committee on Occupational Standards. Draft March 1996.

Dillon WR, Goldstein M: Multivariate analysis. Methods and applications. New York, John Wiley \& Sons, 1984.

Dinarello CA, Savage N. Interleukin-1 and its receptor. Crit Rev Immunol 1989;9:1-20.

Djeu JY, Serbousek D, Blanchard DK. Release of tumor necrosis factro by human polymorphonuclear leukocytes. Blood 1990;76:1405-9.

Driscoll KE, Carter JM, Howard BW, Hassenbein DG. Mutagenesis in rat lung epithelial cells after in vivo silica exposure or ex vivo exposure to inflammatory cells. Am J Respir Crit Care Med 1994;149:A553.

Driscoll KE, Hassenbein DG, Carter J, Poynter J, Asquith TN, Grant RA, Whitten J, Purdon MP, Takigiku R. Macrophage inflammatory proteins 1 and 2: Expression by rat alveolar macrophages, fibroblasts, and epithelial cells in rat lung after mineral dust exposure. Am J Respir Cell Mol Biol 1993;8:311-28.

Driscoll KE. Macrophage inflammatory proteins: Biology and role in pulmonary inflammation. Exp Lung Res 1994;20:473-90.

Driscoll KE, Hassenbein DG, Carter J, Poynter J, Asquith TN, Grant RA, Whitten J, Purdon MP, Takigiku R. Macrophage inflammatory proteins 1 and 2: Expressicin by rat alveolar macrophages, fibroblasts, and epithelial cells in rat lung after mineral dust exposure. Am J Respir Cell Mol Biol 1993; 8: 311-8.

Driscoll KE, Lindenschmidt RC, Maurer JK, Perkins L, Perkins M, Higgins J. Pulmonary response tn inhaled silica or titanium dioxide. Toxicol Appl Pharmacol 1991;111:201-10.

Driscoll KE, Lindenschmidt RC, Maurer JK, Higgins JM, Ridder G. Pulmonary response to silica or titanium-dioxide: Inflammatory cells, alveolar macrophage-derived cytokines, and histopathology. Am J Respir Cell Mol Biol 1990; 2: 381-90.

Dubar V, Gosset P, Aerts C, Voisin C, Wallaert B, Tonnel AB. In vitro acute effects of tobacco smoke on tumor necrosis factor- $\alpha$ and interleukin- 6 production by alveolar macrophages. Exp Lung Res 1993;19:345-59.

Dubois CM, Bissonnette E, Rola-Pleszczynski M. Asbestos fibers and silica particles stimulate rat alveolar macrophages to release tumor necrosis factor. Autoregulatory role of leukotriene B4. Am Rev Respir Dis 1989;139:1257-64.

Dutch Health Council/Gezondheidsraad: Commissie Silicosediagnostiek. Diagnostiek van silicose. Den Haag: Gezondheidsraad, 1992, n. 17. 
Filits JA, Russman MD, Daniele RP. Blood and lung mononuclear cell inhibition of fibroblast growth in sarcoidosis. Am Rev Respir Dis 1984;130:1050-7.

Elias IA, Zurier KB, Rossman MD, Berube ML, Daniele RP. Inhibition of lung fibroblast growth by human lung mononuclear cells. Am Rev Respir Dis 1984;130:810-6.

Elias JA, Jimenez $\$ A$, Freundlich B. Recombinant gamma, alpha, and beta interferon regulation of human lung fibroblast proliferation. Am Rev Respir Dis 1987;135:62-5.

Elias JA, Gustilo K, Baeder W, Freundlich B. Synergistic stimulation of fibroblast prostaglandin production by recombinant interleukin 1 and tumor necrosis factor. J Immunol 1987;138:3812-6.

Elias JA, Gustilo K, Freundlich B. Human alveolar macrophage and blood monocyte inhibition of fibroblast proliferation. Evidence for the synergy between interleukin 1 and tumor necrosis factor. Am Rev Respir Dis 1988;138:1595-603.

Elias JA, Rossman MD, Zurier RB, Daniele RP. Human alveolar macrophage inhibition of lung fibroblast growth. A prostaglandin-dependent process. Am Rev Respir Dis 1985;131:94-9.

Elias JA, Freundlich B, Kem JA, Rosenbloom J. Cytokine networks in the regulation of inflammation and fibrosis in the lung. Chest 1990,97:1439-45.

Elias JA, Zitnik RJ. Cytokine-cytokine interactions in the context of cytokine networking. Am J Respir Cell Mol Biol 1992,7:365-7.

Engelen JJM, Borm PJA, van Sprundel M, Lenaerts L. Blood-anti-oxidant parameters at different stages of pneumoconiosis. Env Health Persp 1990;84:165-72.

Enterline PE. Mortality rates among coal miners. Am J Public Health 1964;54:758-68.

Ipler GR, MCLoud TC, Gaensler EA, Mikus JP, Carrington CB. Normal chest roentgenograms in chronic infiltrative lung disease. N Engl J Med 1978;298:934-9.

Espevik T, Figari IS, Shalaby MR, Lackides GA, Lewis GD, Shepard HM, Palladino MA Jr. Inhibition of cytokine production by cyclosporin $A$ and transforming growth factor beta. I Exp Med 1987;160:571-6.

Evelo CTA, Bos RP, Borm PJA. Decreased glutathione content and glutathione-S-transferase astivity in red blood cells of coal miners with early stages of pneumoconiosis. Br J Ind Med 1993;50:6.3.3-6.

Evirson Ml', Chandler DB. Changes in distribution, morphology, and tumor necrosis factor- $\alpha$ secretion of alveolar macrophage subpopulations during the development of bleomycin-induced pulmonary fibrosis. Am J Pathol 1992;140:503-12.

Fabisiak JP, Absher M, Evans JN, Kelley J. Spontaneous production of PDGF A-chain homodimer by rat lung fibroblasts in vitro. Am J Physiol 1992;263:L185-93.

Fine A, Goldstein RH. The effect of transforming growth factor-beta on cell proliferation and collagen production by lung fibroblasts. J Biol Chem 1987;262:3897-902.

Fletcher CM. Pneumoconiosis of coal-miners. Br Med J 1948;1:1015-1022, 1064-1074.

Fletcher CM, Pride NB. Definitions of emphysema, chronic bronchitis, asthma and chronic airflow obstruction: 25 years on from the Ciba symposium. Thorax 1984;39:81-5.

Flint A. Pathologic features of interstitial lung diseases. In: Schwartz MI, King TE, Eds. Interstitial lung diseases, BC Dekker Inc. Toronto, 1988, pp. 45-62.

Floyd RA, Watson JJ, Wong PK, Atmiller DH, Rickard F. Hydroxyl free radical adduct of deoxyguanosine: sensitive detection and mechanisms of formation. Free Rad Res Comm 1986;1: $163-72$.

Foote CS, Denny RW. Chemistry of singlet oxygen. VII. Quenching by B-carotene. J Am Chem Soc 1968;90:6233-5.

François P, Prevost J, Courtois G, Mas A. Pneumoconiosis of delayed apparition: large scaled screening in a population of retired coal miners of the northern coal fields of France. In: Proceedings of the VIIIth International Pneumoconioses Conference, Pittsburgh, Pennsylvania USA, NIOSH, 90-108 part II, 1988, pp. 979-84.

Frankenberger $M$, Sternsdorf T, Pechumer $H$, Pforte A, Ziegler-Heitbrock HW. Differential cytokine expression in human blood monocyte subpopulations: a polymerase chain reaction analysis. Blood 1996;87:373-7. 
Fridovich I. Editorial: Superoxide radical and the bactericidal action of phagocytes. N Engl J Med 1974;290:624-5.

Fukuda Y, Ferrans VJ, Schoenberger CI, Rennard SI, Crystal RG. Patterns of pulmonary structural remodeling after experimental paraquat toxicity. The morphogenesis of intraalveolar fibrosis. Am J Pathol 1985;118:452-7.

Fuhlbrigge RC, Chaplin DD, Kiely JM, Unanue ER. Regulation of interleukin 1 gene expression by adherence and lipopolysaccharide. J Immunol 1987;138:3799-802.

Gadek JE, Fells GA, Wright DG, Crystal RG. Human neutrophil elastase functions as a type III collagen "collagenase". Biochem Biophys Res Commun 1980;95:1815-22.

Gadek JE, Kelman JA, Fells G, Weinberger SE, Horwitz AL, Reynolds HY, Fulmer JD, Crystal RG. Collagenase in the lower respiratory tract of patients with idiopathic pulmonary fibrosis. $\mathrm{N}$ Engl J Med 1979;301:737-42.

Gaensler EA, Cardigan JB, Sasahara AA, Fox EO, MacMahon HE. Graphite pneumoconiosis of electrotypers. Am J Med 1966;41:864-82.

Gamble JR, Harlan JM, Klebanoff SJ, Vadas MA. Stimulation of the adherence of neutrophils to umbilical vein endothelium by human recombinant tumor necrosis factor. Proc Natl Acad Sci USA 1985;82:8667-71.

Garbisa S, Ballin M, Daga-Gordini D, Fastelli G, Naturale M, Negro A, Semenzato G, Liotta LA. Transient expression of type IV collagenolytic metalloproteinase by human mononuclear phagocytes. J Biol Chem 1985;261:2369-75.

Gauldie J, Northemann W, Fey GH. IL-6 functions as an exocrine hormone in inflammation. Hepatocytes undergoing acute phase responses require exogenous IL-6. J Immunol 1990;144:38048.

Gautrin D, Auburtin G, Alluin F, Brice F-M, Chouraki B, François F, Marquet M, Pouré C, Sénécot B, Szmacinski R, Vandeweghe S, Sébastien P. Recognition and progression of Coal Workers' Pneumoconiosis in the colleries of Northern France. Exp Lung Res 1994;20:395-410.

Gery I, Gershon RK, Waksman BH. Potentiation of the T-lymhocyte response to mitogens. I. The responding cell. J Exp Med 1972;136:128-42.

Gery I, Waksman BH. Potentiation of the T-lymhocyte response to mitogens. II. The cellular source: of potentiating mediatore(s). J Exp Med 1972;143-55.

Gery I, Davies P, Derr J, Krett N, Barranger JA. Relationship between production and release of lymphocyte-activating factor (interleukin-1) by murine macrophages. 1 Effects of various: agents. Cell Immunol 1981;64:293-303.

Ghio AJ, Kennedey TP, Schapira RM, Crumbliss AL, Hoidal JR. Hypothesis: is lung disease after silicate inhalation caused by oxidant generation. Lancet 1990;336:967-9.

Gilligan DM, O'Connor CM, Ward K, Moloney D, Bresnihan B, FitzGerald MX. Bronchoalveolar lavage in patients with mild and severe rheumatoid lung disease. Thorax 1990;45:591-6.

Giri SN, Schwartz LW, Hollinger MA, Freywald ME, Schiedt MJ, Zuckerman JE. Biochemical and structural alterations of hamster lungs in response to intratracheal administration of bleomycin. Exp Mol Pathol 1980;33:1-4.

Goldring MB, Krane SM. Modulation of recombinant interleukin 1 of synthesis of types I and III collagens and associated procollagen mRNA levels in cultured human cells. J Biol Chem 1987;262:16724-9.

Goldstein IM, Kaplan HB, Edelson HS, Weissmann G. Ceruloplasmin. A scavenger of superoxide anion radicals. J Biol Chem 1979:254:4040-5.

Goldstein RH, Fine A. Potential therapeutic initiatives for fibrogenic lung diseases. Chest 1995; 108:848-55.

Goodglick LA, Kane AB. Role of reactive oxygen metabolites in crocidolite asbestos toxicity to mouse macrophages. Cancer Res 1986;46:5558-66.

Gosset P, Lasalle P, Vanhée D, Wallaert B, Aerts C, Voisin C, Tonnel AB. Production of tumor necrosis factor- $\alpha$ and interleukin- 6 by human alveolar macrophages exposed in vitro to coalmine dust. Am J Respir Cell Mol Biol 1991; 5: 431-6. 
Grandjean P, Brown SS, Reavey P, Young DS. Biomarkers of chemical exposure: State of the art. Clin Chem 1994;40:1360-2.

Gross NJ. Pulmonary effects of radiation therapy. Ann Intern Med 1977;86:81-92.

Gross P. The mechansisms of some structural alterations of the lung. Arch Occup Health 1967;14:883-91.

Gross P. Pneumoconioses. In: Parmeggiani L, Ed. Encyclopaedia of Occupational Health and Safety, International Labour Office, Geneva 1983, pp. 1731-33.

Grotendorst GR, Smale G, Pencev D. Production of transforming growth factor beta from peripheral blood monocytes and neutophils. J Cell Physiol 1989;140:396-402.

Gupta RC. Nonrandom binding of the carcinogen N-hydroxy-2-acetyl-aminofluorene to repetitive sequences of rat liver DNA in vivo. Proc Natl Acad Sci 1984;81;6934-47.

Haber F, Weiss J. The catalytic decomposition of hydrogen peroxide by iron salts. Proc $\mathrm{R}$ Soc London 1934;147:332-51.

Habig WH, Jacobi WB. Assays for the differentiation of glutathione-S-transferases. Methods Enzymol 1981;77:398-405.

Halliwell B. Albumin, an important extracellular antioxidant? Biochem Pharmacol 1988;37:56971.

Halliwell B, Cross CE. Oxygen-derived species: Their relation to human disease and environmental stress. Env Health Perspect 1994;102( Suppl 10):5-12.

Hanoa R. Graphite pneumoconiosis: A review of ethiologic and epidemiologic aspects. Scand J Work Environ Health 1983;9:303-14.

Hanaoka T, Tsugane S, Yamano $Y$, Takahashi T, Kasai $H$, Natori $Y$, Watanabe S. Quantitative analysis of 8-hydroxyguanine in peripheral blood cells: An application for asbestosis patients. Int Arch Occup Environ Health 1993;65:S215-17.

Hance AJ, Bradley K, Crystal RG. Lung collagen heterogeneity. Synthesis of type I and type III collagen by rabbit and human lung cells in culture. J Clin Invest 1976;57:102-11.

Hanley JA, MCNeil BJ. The meaning and use of the area under a receiver operating characteristic (ROC) curve. Radiology 1982;143:29-36.

Hansen K, Mossman BT. Generation of suproxide (O2-.) from alveolar macrophages exposed to asbestiform and nonfibrous particles. Cancer Res 1987;47:1681-6.

Hart CE, Forstrom JW, Kelly JD, Seifert RA, Smith RA, Ross R, Murray MJ, Bowen-Pope DF. Two classes of PDGF receptor recognize different isoforms of PDGF. Science 1988;240:1529-31.

Hartmann DP, Georgian MM, Oghiso Y, Kagan E. Enhanced interleukin-1 activity following asbestos inhalation. Clin Exp Immunol 1984;55:643-50.

Henderson RF, Harkema JR, Hotchkiss JA, Boehme DS. Effect of blood leukocyte depletion on the inflammatory response of the lung to quartz. Toxicol Appl Pharmacol 1991:109:127-36.

Heppleston AG. Coal workers' pneumoconiosis: A historical perspective on its pathogenesis. Am J Ind Med 1992;22:905-23.

Heppleston AG, Styles JA. Activity of a macrophage factor in collagen formation by silica. Nature 1967;214:521-2.

Hesterberg TW, Oshimura M, Brody AR, Barrett JC. Asbestos and silica induce morphological transformation of mammalian cells in culture: a possible mechanism. In: Goldsmith DF, Winn DM, Shy CM, Eds. Silica, silicosis and cancer. Praeger, New York, 1986, pp. 177-90.

Hibbs MS, Hoidal JR, Kang AH. Expression of a metalloproteinase that degrades native type V collagen and denatured collagens by cultured human alveolar macrophages. J Clin Invest 1987;80:1644-50.

Hissin PJ, Hilf P. A fluorometric method for the determination of oxidized and reduced glutathione in tissues. Anal Biochem 1976;74:214-26.

Holland LM. Crystalline silica and lung cancer: A review of recent experimental evidence. Regul Toxicol Pharmacol 1990;12:224-37.

Honda K, Hirayama K, Kikuchi I, Nagato H, Tamai H, Sasazuki T. HLA and silicosis in Japan (letter). N Engl J Med 1988;319:1610. 
Horwitz AL, Hance AJ, Crystal RG. Granulocyte collagenase: selective digestion of type I relative to type III collagen. Proc Natl Acad Sii USA 1977;74:897-9(1).

Hoyt DG, Lazo JS. Bleomycin and cyclophosphamide increase pulmonary type IV prucullagen mRNA in mice. Am J Physiol 1990;259:L47-52.

Huang X, Zalma R, Pezerat $H$. Factors that influence the formation and stability of hydrated ferrous sulfate in coal dusts. Possible relation to the emphysema of coal miners. Chem Res Toxicol 1994;7:451-7.

Hubbard RC, Fells G, Gadek J, Pacholok S, Humes J, Crystal RG. Neutrophil accumulation in the lung in alpha 1-antitrypsin deficiency. Spontaneous release of leukotriene B4 by alvenlar macrophages. J Clin Invest 1991;88:891-7.

Hunninghake GW, Kalica AR. Approaches to the treatment of pulmonary fibrosis. Am J Respir Crit Care Med 1995;151:915-8.

Hurley JF, Alexander WP, Hazledine DJ, Jacobsen M, MacLaren WM. Exposure to respirable coalmine dust and incidence of progressive massive fibrosis. $\mathrm{Br} J$ Ind Med 1987;44:661-72.

Hyde DM, Henderson TS, Giri SN, Tyler NK, Stovall MY. Effect of murine gamma interferon on the cellular responses to bleomycin in mice. Exp Lung Res 1988;14:687-704.

IARC. Evaluation of the carcinogenic risk of chemicals to humans: silica and some silicates, 1987, Vol 42. International Agency for Research on Cancer, Lyon, France.

Ignotz RA, Endo T, Massague J. Regulation of fibronectin and type I collagen mRNA levels by transforming growth factor-beta. J Biol Chem 1987;262:6443-6.

Ignotz RA, Massague J. Transforming growth factor-beta stimulates the expression of fibronectin and collagen and their incorporation into the extracellular matrix. J Biol Chem 1986;261:4337-45.

International Labour Office: Guidelines for the use of ILO international classification of radiographs of pneumoconiosis. Revised edition. Occupational Safety and Health series No 22. Geneva, 1980.

Jäätelä M. Biology of disease. Biologic activities and mechanisms of action of tumor necrosis factor$\alpha /$ cachectin. Lab Invest 1991;64:724-42.

Jacobsen M, Rae S, Walton WH, Rogan JM. New dust standards for British coal miners. Nature 1970;227:445-447.

Jakobsson K, Rannug A, Alexandrie AK, Rylander L, Albin M, Hagmar L. Genetic polymorhpism for glutathione-S-transferase mu in asbestos cement workers. Occup Environ Med 1994;51:812-6.

Janoff A, Carp H. Proteases, antiprotease, and oxidants: pathways of tissue injury during inflammation. Monogr Pathol 1982;62-82.

Janoff A. Elastases and emphysema. Current assessment of the protease-antiprotease hypothesis. Am Rev Respir Dis 1985;132:417-33.

Janssen YMW, Engelen JJM, Giancola MS, Low RB, Vacek P, Borm PJA. Serum type III procollagen $\mathrm{N}$-terminal peptide in coal miners. Exp lung Res 1992a;18:1-8.

Janssen YMW, Marsh JP, Absher MP. Hemenway D, Vacek PM, Leslie KO, Borm PJA, Mossman BT. Expression of antioxidant enzymes in rat lungs after inhalation of asbestos or silica. J Biol Chem 1992b;267:10625-30.

Janssen YMW, Borm PJA, van Houten B, Mossman BT. Cell and tissue responses to oxidative damage. Lab Invest, 1993;69:261-74

Janssen YMW, Marsh JP, Driscoll KE, Borm PJA, Oberdörster G, Mossman BT. Increased expression of manganese-containing superoxide dismutase in rat lungs after inhalation of inflammatory and fibrogenic minerals. Free Rad Biol Med 1994a;16:315-22.

Janssen YMW, Heintz NH, Marsh JP, Borm PJA, Mossman BT. Induction of c-fos and c-jun protooncogenes in target cells of the lung and pleura by carcinogenic fibers. Am J Respir Cell Mol Biol 1994b;11:522-30.

Jones RN, Diem JE, Hughes JM, Hammad YY, Glindmeyer HW, Weill H. Progression of asbestos effects: A prospective longitudinal study of chest radiographs and lung function. $\mathrm{Br} J$ Ind Med 1989;46:97-105.

Jorna THJM, Schins RPF, Lenaerts L, Derhaag TJJM, Wouters EFM, Borm PJA. Airflow obstruction and monocyte TNF release in coal workers. Exp Lung Res 1994a;20:421-31. 
Jorna THJM, Borm PJA, Koiter KD, Slangen JJM, Henderson PTh, Wouters EFM. Respiratory effects and serum type IIII procollagen in potato sorters exposed to diatomaceous earth. Int Arch Occup Environ Health, 1994b;66:217-27.

Kähäri VM, Chen YQ, Su MW, Ramirez F, Uitto J. Tumor necrosis factor-alpha and interferongamma suppress the activation of human type I collagen gene expression by transforming growth factor-beta 1. J Clin Invest 1990;86:1489-95.

Kamijo R, Takeda K, Nagumo M, Konno K. Suppression of TNF-stimulated proliferation of diploid fibroblasts and TNF-induced cytotoxicity against transformed fibroblasts by TGF-beta. Biochem Biophys Res Commun 1989;158:155-62.

Kamp DW, Graceffa P, Pryor W, Weitzman S. The role of free radicals in asbestos-induced diseases. Free Radic Biol Med 1992;12:293-315.

Karagianes MT, Palmer RF, Bush RH. Effects of inhaled diesel emissions and coal dust in rats. Am Ind Hyg Assoc J 1981;42:38291.

Kasai H, Crain PF, Kuchino Y, Nishimura S, Octsuyama A, Tanocka H. Formation of 8hydroxyguanine moiety in cellular DNA by agents producing oxygen radicals and evidence for its repair. Carcinogenesis 1986;7:1849-51.

Kauffmann F, Drouet D, Lellouch J, Brille D. Occupational exposure and 12 year spirometric changes among Paris area workers. Br J Ind Med 1982;39:221-32.

Kehrer JP. Free radicals as mediators of tissue injury and disease. Crit Rev Toxicol 1993;32:21-48.

Keller GA, Warner TG, Steimer KS, Halliwell RA. CuZn superoxide dismutase is a peroxisomal enzyme in human fibroblast and hepatoma cells. Proc Natl Acad Sci USA 1991;88:7381-5.

Kelley J, Shull S, Walsh JJ, Cutroneo KR, Absher M. Auto-induction of transforming growth factorbeta in human lung fibroblasts. Am J Respir Cell Mol Biol 1993;8:417-24.

Kelley J. Cytokines of the lung. Am Rev Respir Dis 1990;141:765-88.

Kensler TW, Trush MA, Role of oxygen radicals in tumor promotion. Environ Mutagen 1984;6:593616.

Khalil N, Bereznay O, Sporn M, Greenberg AH. Macrophage production of transforming growth factor- $\beta$ and fibroblast collagen synthesis in chronic pulmonary fibrosis. J Exp Med 1989;170:72737

Kibelstis JA, Morgan EJ, Reger R, LeRoy, Lapp N, Seaton A, Keith W, Morgan C. Prevalence of bronchitis and airway obstruction in American bituminous coal miners. Am Rev Respir Dis 1973;108:886-93.

Klebanoff SJ. Myeloperoxidase-halide-hydrogen peroxide antibacterial system. J Bacteriol 1968;95:2313-8.

Klebanoff SJ. Oxygen metabolism and the toxic properties of phagocytes. Ann Intern Med 1980;93:480-9.

Klebanoff SJ, Vadas MA, Harlan JM, Sparks LH Gamble JR, Agosti JM, Waltersdorph AM. Stimulation of neutrophils by tumor necrosis factor. J Immunol 1986;136:4220-5.

Kleinfeld K, Messite J, Giel JP. Talc and Related Lung Diseases. In: Rom, Ed. Environmental and Occupational Medicine, Little, Brown and Company, Boston, 2nd edition, 1992, pp. 301-6.

Kotloff RM, Little J, Elias JA. Human alveoalar macrophage and blood monocyte interleukin- 6 production. Am J Respir Cell Mol Biol 1990;3:497-505.

Kuchino $Y$, Mori F, Kasai $H$, Inoue $H$, Iwai S, Miura K, Ohtoka E, Nishimura S. Misreading of DNA templates containing 8-hydroxydeoxyguanosine at the modified base and at adjacent residues. Nature 1987;32:77-9.

Kuhn DC, Demers LM. Influence of mineral dust surface chemistry on eicosanoid production by the alveolar macrophage. J Toxicol Environ Health 1992;35:39-50.

Kunkel SL, Spengler M, May MA, Spengler R, Larrick J, Remick D. Prostaglandin E2 regulates macrophage-derived tumor necrosis factor gene expression. J Biol Chem 1988;263:5380-4.

Lamers RJS, Schins RPF, Borm PJA, Wouters EFM, van Engelshoven JMA. High Resolution Computed tomography of the lungs in coal miners with a normal chest radiograph. Exp Lung Res 1994;20:411-9.

Landrigan PJ. Silicosis. Occup Med 1987;2:319-26.

166 
Lapp NL, Castranova V. How silicosis and coal workers' pneumoconiosis develop. A cellular assessment. Occup Med 1993;8:35-56.

Lapp NL, Morgan WK, Zaldivar G. Airways, obstruction, coal mining, and disability. Occup Environ Med 1994;51:234-8.

Lasalle P, Gosset P, Aerts C, Fournier E, Lafitte JE, Degreef JM, Wallaert B, Tonnel AB, Voisin C. Abnormal secretion of interleukin- 1 and tumor necrosis factor- $\alpha$ by alveolar macrophages in coal workers' pneumoconiosis: comparison between simple pneumoconiosis and progressive massive fibrosis. Exp Lung Res 1990;16:73-80.

Laskin DL, Kimura T, Sakakibara S, Riley DJ, Berg RA. Chemotactic activity of collagen-like polypeptides for human peripheral blood neutrophils. J Leukoc Biol 1986;39:255-66.

Latini R, Bianchi M, Correale E, Dinarello CA, Fantuzzi G, Fresco C, Maggioni AP, Mengozzi M, Romano S, Shapiro L, Sironi M, Tognoni G, Turano R, Ghezzi P. Cytokines in acute myocardial infarction: Selective increase in circulating tumor necrosis factor, its soluble receptor, and interleukin-1 receptor antagonist. J Cardiovasc Pharamcol 1994;23:1-6.

Laurent GJ, McAnulty RJ. Protein metabolism during bleomycin-induced pulmonary fibrosis in rabbits. In vivo evidence for collagen accumulation due to increased synthesis and decreased degradation of the newly synthesized collagen. Am Rev Respir Dis 1983;128:82-8.

Leanderson P, Söderkvist P, Tagesson C, Axelson O. Formation of 8-hydroxydeoxyguanosine by asbestos and man made mineral fibres. $\mathrm{Br}$ J Ind Med 1988;45:309-11.

Leeuwenberg JFM, Dentener MA, Buurman WA. Lipopolysaccharide LPS-mediated soluble TNF receptor release and TNF receptor expression in by monocytes. Role of CD14, LPS binding protein, and bactericidal/permeability-increasing protein. J Immunol 1994; 152: 5070-76.

Leeuwenberg JFM, Jeunhomme TMAA, Buurman WA. Slow release of soluble TNF receptors by monocytes in vitro. J Immunol 1994; 152: 4036-4043.

Leff JA, Parsons PE, Day CE, Taniguchi N, Jochum M, Fritz H, Moore FA, McCord JM, Repine JE. Serum antioxidants as predictors of adult respiratory distress syndrome in patients with sepsis. Lancet 1993;341: 777-80.

Leibovich SJ, Ross R. A macrophage-dependent factor that stimulates the proliferation of fibroblasts in vitro. Am J Pathol 1976;84:501-14.

Leibovich SJ, Polveniri PJ, Shepard HM, Wiseman DM, Shively V, Nuseir N. Macrophage-induced angiogenesis is mediated by tumor necrosis factor-alpha. Nature 1987;329:630-2.

Leof EB, Proper JA, Goustin AS, Shipley GD, DiCorleto PE, Moses HL. Induction of c-sis mRNA and activity similar to platelet-derived growth factor by transforming growth factor-beta: a proposed model for indirect mitogenesis involving autocrine activity. Proc Natl Acad Sci USA 1986; 83:2453-7.

Liddell FN. Validation of the UICC/Cincinnati classification of radiographs in terms of prediction of mortality of asbestos workers. LARC Sci Publ 1980, vol 30, pp. 667-71.

Linden $M$, Rasmussen JB, Piitulainen E, Tunek A, Larson $M$, Tegner $H$, Venge P, Laitinen LA, Brattsand R. Airway inflammation in smokers with nonobstructive chronic bronchitis. Am Rev Respir Dis 1993; 148:1226-32.

Lindroos PM, Coin PG, Osornio-Vargas AR, Brody AR. Interleukin-1 beta and the IL-1 beta-alpha 2-macroglobulin complex upregulate the platelet-deived growth factor alpha-receptor on rat alveolar pulmonary fibroblasts. Am J Respir Cell Mol Biol 1995;13:455-65.

Lippmann M. Asbestos exposure indices. Environ Res 1988;46:86-106.

Loetscher H, Pan YE, Lahm H-W, Gentz R, Brockhaus M, Tabuchi H, Lesslauer W. Molecular cloning and expression of the human $55 \mathrm{kd}$ tumor necrosis factor receptor. Cell 1990;61:351-9.

Love RG, Miller BG. Longitudinal study of lung function in coal miners. Thorax 1982;37:193-197.

Low RB, Giancola MS, King TE, Chapitis J, Vacek P, Davis GS. Serum and bronchoalveolar lavage of N-terminal type III procollagen peptides in idiopathic pulmonary fibrosis. Am Rev Respir Dis 1992;146:701-6.

Low RB, Cutroneo KR, Davis GS, Giancola MS. Lavage type III procollagen N-terminal peptides in human pulmonary fibrosis and sarcoidosis. Lab Invest 1983;48:755-9. 
Luyano EM, Datuber JH, Elias JA, Bashey RI, Jimenez SA, Daniele RP. The regulation of lung tibroblast proliferation by alveolar macrophages in experimental silicosis. An Rev Respir Dis 1984;129:767-71.

Luisetti M, Bulgheroni A. Elevated serum procollagen III aminopeptide levels in sarcoidosis. Chest 1990;98:1414-20.

Lutgerink JT, van de Akker E, Smeets L, Pachen D, van Dijk P, Aubry J-M, Joenje H, Lafleur V, Retel J. Interaction of singlet oxygen with DNA and biological consequences. Mut Res 1992;275:377-86.

Lyons JP, Ryder RC, Seal RM, Wagener JC. Enphysema in smoking and in non-smoking coalworkers with pneumoconiosis. Bull Eur Physiopathol Respir 1981;17:75-85.

I yons RM, Keski-Oja-J, Moses HL. Proteolytic activation of latent transforming growth factor-beta from fibroblast-conditioned medium. J Cell Biol 1988;106:1659-65.

MacLaren WM, Soutar CA. Progressive massive fibrosis and simple pneumoconiosis in ex-miners. $\mathrm{Br}$ J Ind Med 1985;42:734-40.

Madri JA, Furthmayr H. Collagen polymorphism in the lung. An immunochemical study of pulmonary fibrosis. Hum Pathol 1980;11: 353-66.

Mahieu B. L'évolution de la pneumoconiose en France au cours des 20 demières années [Corrigendum exposé]. Hygiene Industrial dans les mines Commision des Communaute europeennes Direction Générale Emploi, Relations Industrielles et Affaires Sociales; Direction Santé et Sécurité $\mathrm{V} / \mathrm{E} / 4,1990$.

Makarak EJ. Collagen synthesis by cloned pulmonary artery endothelial cells. J Cell Physiol 1984;119:175-82.

Malmberg P, Hedenstrom H, Sundblad BM. Changes in lung function of granite crushers exposed to moderately high silica concentrations: a 12 year follow-up. Br J Ind Med 1993;50:726-31.

Maral J, Puget K, Michelson M. Comparative study of superoxide dismutase, catalase and glutathione peroxidase in erythrocytes of different animals. Bioche Biophys Res Commun $1977 ; 77: 1725-35$.

Marine, WM, Gurr D, Jacobsen M. Clinically important respiratory effects of dust exposure and smoking in Britisch coal miners. Am Rev Respir Dis 1988;137:106-12.

Marklund SL. Extracellular superoxide dismutase and other superoxide dismutase isoenzymes in tissueas from nine mammalian species. Biochem J 1984;222:649-55.

Marsh JP, Mossman BT. Role of asbestos and active oxygen species in activation and expression of ornithine decarboxylase in hamster tracheal epithelial cells. Cancer Res 1991;51:167-73.

Martinet Y, Rom WN, Grotendorst GR, Martin GR, Crystal RG. Exaggerated spontaneous release of platelet-derived growth factor by alveolar macrophages from patients with idiopathic pulmonary fibrosis. N Engl J Med 1987;317:202-9.

Martinet Y, Yamauchi K, Crystal RG. Differential expression of the tumor necrosis factor/cachectin gene by blood and lung mononuclear phagocytes. Am Rev Respir Dis 1988;138:659-665.

Martin JC, Daniel H, LeBouffant L. Short and longterm experimental study of the toxicity of coal mine dust and some of its constituents. Inhaled Part 1975;4:361-71.

Matolo NM, Gorishek WM, Moslander V, Dixon A. Coal mining and cancer of the stomach. Rocky Mt Med J 1972;69:44-9.

Mauderly JL, Samet JM. General environment. In: Crystal RG, West JB, Barnes PJ, Cherniack NS, Weibel ER, Eds. The lung. Scientific Foundations. Raven Press, New York, 1991, pp. 1947-60.

May LT, Torcia G, Cozzolino F, Ray A, Tatter SB, Santhanam U, Sehgal PB, Stern D. Interleukin-6 gene expression in human endothelial cells: RNA start sites, multiple IL-6 proteins and inhibition of proliferation. Biochem Biophys Res Commun 1989;159:991-8.

McCord JM, Fridovich I. Superoxide dismutase. An enzymatic function for erythrocuprein (hemocuprein). J Biol Chem 1969;244:6049-55.

McDonald G. Piggot AP, Gilder FW. Two cases of acute silicosis. Lancet 1930;2:846-7.

McDonald JC. Silica, silicosis and lung cancer. Br J Ind Med 1989;46:289-91.

Meier B, Radeke HH, Selle S, Younes M, Sies H, Resch K, Habermehl GG. Human fibroblasts release reactive oxygen species in response to interleukin-1 or tumor necrosis factor-alpha. Biochem J 1989;263:539-45. 
Meijers JMM. Exposure to inorganic dust and occupational diseases. Epidemiological investigations and methods. 1991, Thesis, Datawyse, Maastricht, pp. 169.

Meijers JMM, Swaen GMH, Slangen JJM, van Vliet C, Sturmans F. Long-term mortality in miners with coal workers' pneumoconiosis in the Netherlands. A pilot study. Am J Ind Med 1991;19:4350 .

Meredith SK, McDonald JC. Work-related respiratory disease in the Unite Kingdom, 1989-1992: report on the SWORD project. Occup Med 1994;44:183-8.

Meyer MB, Luk GD, Sotelo JM, Cohen BH, Menkes HA. Hypothesis: The role of the lung in stomach carcinogenesis. Am Rev Respir Dis 1980;121:887-92.

Merchant JA, Schwartz DA. Chest radiography for assessment of the pneumoconioses. In: Rom, Ed. Environmental and Occupational Medicine, Little, Brown and Company, Boston, 2nd edition, 1992, pp. 215-25.

Miller BG, Jacobsen M. Dust exposure, pneumoconiosis, and mortality of coal miners. Br J Ind Med 1985;42:723-33.

Ming WJ, Bersani L, Mantovani A. Tumor necrosis factor is chemotactic for monocytes and polymorphonuclear leukocytes. J Immunol 1987;138:1469-74.

Mohr C, Gemsa D, Graebner C, Hemenway DR, Leslie KO, Absher PM, Davis GS. Systemic Macrophage stimulation in rats with silicosis: Enhanced release of tumor necrosis factor- $\alpha$ from alveolar and peritoneal macrophages. Am J Respir Cell Mol Biol 1991;5:395-402.

Mohr C, Davis GS, Graebner C, Hemenway DR, Gemsa D. Enhanced release of prostaglandin E2 from macrophages of rats with silicosis. Am J Respir Cell Mol Biol 1992;6:390-6.

Mohr C, Davis GS, Graebner C, Amann S, Hemenway DR, Gemsa D. Reduced release of leukotrienes B4 and C4 from alveolar macrophages of rats with silicosis. Am J Respir Cell Mol Biol 1992;7: 542-7.

Mölvig J, Baek L, Christensen P, Manogue KR, Vlassara H, Platz P, Nielsen LS, Svejgaard A, Nerup $J$. Endotoxin-stimulated human monocyte secretion of interleukin-1, tumour necrosis factor alpha, and prostaglandin E2 show stable interindividual differences. Scand J Immunol 1988;27:705-716.

Morabia A, Sorenson A, Kumanyika SK, Abbey H, Cohen BH, Chee E. Vitamin A, cigarette smoking, and airway obstruction. Am Rev respir Dis 1989; 140:1312-6.

Morabia A, Markowitz S, Garibaldi K, Wynder EL. Lung cancer and occupation: results of a multicentre case-control study. Br J Ind Med 1992;49:721-7.

Morgan WK, Lapp NL. Respiratory disease in coal miners. Am Rev Respir Dis 1976;113:531-59.

Morrow PE. Dust overloading of the lungs: update and appraisal. Toxocol Appl Pharmacol 1992;113:1-12.

Moses HL, Yang EY, Pietenpol JA. TGF-beta stimulation and inhibition of cell proliferation: new mechanistic insights. Cell 1990;63:245-7.

Mossman BT, Marsh JP, Shatos MA. Alterations of superoxide dismutase activity in tracheal epithelial cells by asbestos and inhibition of cytotoxicity by antioxidants. Lab Invest $1986 ; 54: 204-12$.

Mossman BT, Marsh JP, Shatos MA, Doherty J, Gilbert R, Hill S. Implications of active oxygen species as second messengers of asbestos toxicity. Drug Chem Toxicol 1987;10:157-80.

Mossman BT, Gee JBL. Medical progress. Asbestos related diseases. N Engl J Med 1989;320:1721-9.

Mossman BT, Bignon J, Corn M, Seaton A, Gee JBL. Asbestos: Scientific developments and implications for public policy. Science 1990;247:294-301.

Mossman BT and Marsh JP. Role of active oxygen species in asbestos-induced cytotoxicity, cell proliferation and carcinogenesis. In: Harris CC, Lechner JF, Brinkley BR, Eds. Cellular and molecular aspects of fiber carcinogenesis. Cold Spring Harbor Laboratory Press, 1991, pp. 159-68.

Muir DC. Particle deposition. In: Crystal RG, West JB, Bames PJ, Cherniack NS, Weibel ER, Eds. The lung. Scientific Foundations. Raven Press, New York, 1991, pp. 1839-43.

Murray JC, Laurent GJ. What is pulmonary fibrosis? Thorax 1988;43:9-11.

National Research Council. Biological markers in environmental health research. Environ Health Perspect 1987;74:1-191. 
Nash T, Allison AC, Harrington JS. Physiochemical properties of silica in relation to its toxicity. Nature 1966;210:259-61.

Naylor MS, Stamp GWH, Foulkes WD, Eccles D, Balkwill FR. Tumor necrosis factor and its receptors in human ovarian cancer. Potential role in disease progression. J Clin Invest 1993; 91: 2194-2206.

Nemery B, van Kerckhoven W, Verbeken EK, Dinsdale D, Demedts M. An unexpected risk of pneumoconiosis in breweries. In: Hurych J, Lesage $M$ and David A, Eds. Proc. 8th International Conference on Occupational Lung diseases, ILO-Geneva and Czech Medical-Soc-Prague, 1992, vol 2, pp. 658-63.

Oberdörster G, Ferin J, Lehnert BE. Correlation between particle size, in vivo particle persistence, and lung injury. Environ Health Perspect 1994;102:173-9.

O'Connor C, Odlum C, van Breda A, Power C, Fitzgerald MX. Collagenase and fibronectin in bronchoalveolar lavage fluid in patients with sarcoidosis. Thorax 1988;43:393-400.

Oghiso $\mathrm{Y}$, Kubota Y. Interleukin 1 production and accessory vcell function of rat alveolar macrophages exposed to mineral dust particles. Microbiol Immunol 1987;31:275-87.

Okutani H, Shima S, Sano T. Graphite pnneumoconiosis in carbon electrode makers. Excerpta Medica 1963;2:626.

Okazaki I, Maruyama K, Okuno F, Suzuki H : Serum type III procollagen peptide in patients with pneumoconiosis. J. UOEH 1983;5:461-467.

Okazaki I, Maruyama K, Kobayashi Y, Lilis R, Suzuki Y. Serum type III procollagen peptide: Indicator for pulmonary fibrosis: II. Application in 80 asbestos insulation workers. Am J Ind Med $1987 ; 11: 439-46$

Osornio-Vargas AR, Kalter VG, Badgett A, Hernández-Rodriguez N, Aguilar-Delfín I, Brody AR. Early-passage rat lung fibroblasts do not migrate in vitro to transfroming growth factor- $\beta$. Am J Respir Cell Mol Biol 1993;8:468-471.

Oxman AD, Muir DC, Shannon HS, Stock SRT, Hnizdo E, Lanfe HJ. Occupational dust exposure and chronic obstructive pulmonary disease. Am Rev Respir Dis 1993;148:38-48.

Pacht ER, Davis WB. Role of transferrin and cerulosplasmin in antioxidant activity of lung epithelial lining fluid. J Appl Physiol 1988;64:2092-9.

Pairon JC, Jaurand MC, Kheuang L, Janson X, Brochard P, Bignon J. Sister chromatid exchanges in human lymphocytes treated with silica. $\mathrm{Br} J$ Ind Med 1990;47:110-5.

Pantelidis P, Southcott AM, Du Bois RM. A comparison of single cell TNF $\alpha$ secretion in blood and lung mononuclear cells in interstitial lung disease. Am J Respir Dis 1993;147:A756.

Parkes. Occuational lung disorders. Butterworhts, London., 1982, pp. 529.

Parmeggiani L. International classification of pneumoconioses. In: Parmeggiani L, Ed. Encyclopaedia of Occupational Health and Safety, International Labour Office, Geneva 1983, pp. 1733-41.

Perera FP, Weinstein IB. Molecular epidemiology and carcinogen-DNA adduct detection: New approaches to studies of human cancer causation. J Chron Dis 1982;35:581-600.

Perkins RC, Scheule RK, Hamilton R, Gomes G, Freidman G, Holian A. Human alveolar macrophage cytokine release in response to in vitro and in vivo asbestos exposure. Exp Lung Res 1993; 19:55-65.

Perrin-Nadif R, Auburtin G, Dusch M, Porcher J-M, Mur J-M. Blood antioxidant enzymes as markers of exposure or effect in coal miners. Occup Environ Med 1996;53:41-5.

Pfeffer KD, Huecksteadt TP, Hoidal JR. Expression and regulation of tumor necrosis factor in macrophages from cystic fibrosis patients. Am J Respir Cell Mol Biol 1993;9:511-9.

Phan SM, Kunkel SL. Lung cytokine production in bleomycin-induced pulmonary fibrosis. Exp Lung Res 1992;18:29-43.

Piguet PF, Collart MA, Grau GE, Kapanci Y, Vassali P. Tumor necrosis factor/cachectin plays a key role in bleomycin-induced pneumopathy and fibrosis. J Exp Med 1989;170:655-63.

Piguet PF, Collart MA, Grau GE, Sappino AP, Vassali P. Requirement of tumor necrosis factor for development of silica-induced pulmonary fibrosis. Nature 1990;344:245-7. 
Piguet PF, Vesin C, Grau GE, Thompson RC. Interleukin 1 receptor antagonist (IL-1ra) prevents or cures pulmonary fibrosis in mice by bleomycin or silica. Cytokine 1993;5:57-61.

Piguet PF, Vesin C. Treatment by human recombinant soluble TNF receptor of pulmonary fibrosis induced by bleomycin or silica in mice. Eur Respir J 1994;7:515-8.

Piguet PF, Vesin C. Pulmonary platelet trapping induced by bleomycin: correlation with fibrosis and involvement of the $\beta 2$ integrins. Int J Exp Path 1994;75:321-8.

Piguet PF, Tacchini-Cottier, Vesin C. Administration of anti-TNF- $\alpha$ or anti-CD1la antibodies to normal adult mice decreases lung and bone collagen content: evidence for an effect on platelet consumption. Am J Respir Cell Mol Biol 1995;12:227-31.

Pierce GF, Mustoe TA, Lingelbach J, Masakowski VR, Griffin GL, Senior RM, Deuel TF. Plateletderived growth factor and transforming growth factor-beta enhance tissue repair activities by unique mechanisms. J Cell Biol 1989;109:429-40.

Pierce GF. Macrophages: important physiologic and pathologic sources of polypeptide growth factors. Am J Respir Cell Mol Biol 1990;2:233-4.

Pociot F, Briant L, Jongeneel CV, Mölvig J, Worsaae H, Abbal M, Thomsen M, Nerup J, CambonThomsen A. Association of tumor necrosis factor (TNF) and class II major histocompatibility complex alleles with the scretion of TNF- $\alpha$ and TNF- $\beta$ by hyman mononuclear cells: a possible link to insulin-dependent diabetes mellitus. Eur J Immunol 1993;23: 224-31.

Pohl WR, Thompson AB, Köhn H, Losch S, Umek H, Legenstein E, Kummer F, Rennard SI, Klech H: Serum procollagen III peptide levels in subjects with sarcoidosis. A 5-year follow-up study. Am Rev Respir Dis 1992;145:412-7.

Porteu F, Hieblot C. Tumor necrosis factor induces a selective shedding of its p75 receptor from human neutrophils. J Biol Chem 1994; 269: 2834-40.

Postlethwaite AE, Kang AH. Collagen-and collagen peptide-induced chemotaxis of human blood monocytes. J Exp Med 1976;143:1299-1307.

Postlethwaite AE, Keski-Oja J, Moses HL, Kang AH. Stimulation of the chemotactic migration of human fibroblasts by transforming growth factor beta. J Exp Med 1987;165:251-6.

Préat B. Synthesis of the results of the ECSC funded research projects on the pneumoconiosis factors. In: Coalworkers' Occupational disease Project. Medical workshop, Hasselt 1-10 sept 1993, Belgium. Book of Abstracts, 1993.

Prior C, Knight RA, Herold M, Ott G, Spiteri MA. Pulmonary sarcoidosis: patterns of cytokine release in vitro. Eur Respir J 1996;9:47-53.

Prockop DJ, Kivirikko KI. Collagens: molecular biology, diseases, and potentials for therapy. Annu Rev Biochem 1995;64:403-34.

Pryor WA. Why is the hydroxyl radical the only radical that commonly adds to DNA? Hypothesis: it has a rare combination of high electrophilicity, high thermochemical reactivity, and a mode of production that can occur near DNA. Free Radic Biol Med 1988;4:219. 23.

Quanjer PH. Standardised lung function testing. Bull Eur Physiopathol Respir 1983;19:7-44.

Rae S, Walker DD, Attfield MD. Chronic bronchitis and dust exposure in British coalminers. Inhaled Part 1970;2:883-96.

Raghow R, Lurie S, Seyer JM, Kang AH. Profiles of steady state levels of messenger mRNAs coding for type I procollagen, elastin, and fibronectin in hamster lungs undergoing bleomycin-induced interstitial pulmonary fibrosis. J Clin Invest 1985;76:1733-9.

Raghow B, Irish P, Kang AH. Coordinate regulation of transforming growth factor beta gene expression and cell proliferation in hamster lungs undergoing bleomycin-induced pulmonary fibrosis . J Clin Invest 1989;84:1836-42.

Raghu G, Striker LJ, Hudson LD, Striker GE. Extracellular matrix in normal and fibrotic human lungs. Am Rev Respir Dis 1985;131:281-9.

Raghu G, Masta S, Meyers D, Narayanan AS. Collagen synthesis by normal and fibrotic human lung fibroblasts and the effect of transforming growth factor-beta. Am Rev Respir Dis 1989;140:95100 . 
Reibman J, Meixler S, Lee TC, Gold LI, Cronstein BN, Haines KA, Kolasinski SL, Weissmann G. Transforming growth factor beta 1, a potent chemoattractant for human neutrophils, bypasses classic signal-transduction pathways. Proc Natl Acad Sci USA, 1991;88:6805-9.

Reisner MTR, Robock K. Results of epidemiological, mineralogical studies on the pathogenicity of coal mine dusts. In: Walton WH, McGovern, Eds. Inhaled Particles IV, Pergamom Press, 1976, pp. 703-15.

Remi-Jardin M, Degreef JM, Beuscart R, Voisin C, Remi J, Coal worker's pneumoconiosis: CT assessment in exposed workers and correlation with radiographic findings. Radiology 1990;177:363-71.

Rennard SI, Hunninghake GW, Bitterman PB, Crystal RG. Production of fibronectin by the human alveolar macrophage: mechanism for the recruitment of fibroblasts to sites of tissue injury in interstitial lung diseases. Proc Natl Acad Sci USA 1981;78:7147-51.

Rennard SI, Jaurand MC, Bignon J, Kawanami O, Ferrans VJ, Davidson J, Crystal RG. Role of mesothelial cells in the production of the submesothelial connective tissue matrix of the lung. Am Rev Respir Dis 1984;130:267-74.

Rhode H, Vargas L, Hahn E, Kalbfleisch H, Bruguera M, Timple R. Radioimmunoassay for type III procollagen peptide and its application to human liver disease. Eur J Clin Invest 1979;9:451

Rihs HP, Lipps P, May-Taube K, Jager D, Schmidt EW, Hegemann JH, Baur X. Immunogenetic studies on HLA-DR in German coal miners with and without coal worker's pneumoconiosis. Lung 1994;172:347-54.

Roberts AB, Anzano MA, Lamb LC, Smith JM, Sporn MB. New class of transforming growth factors potentiated by epidermal growth factor: isolation from non-neoplastic tissues. Proc Natl Acad Sci USA 1981;78:5339-43.

Rogan JM, Attfield MD, Jacobsen M, Rae S, Walker DD, Walton WH. Role of dust in the working environment in development of chronic bronchitis in British coal miners. Br J Ind Med 1973;30:217-26.

Rom WN. The discipline of Environmental and Occupational Medicine. In: Rom WN, Ed. Environmental and Occupational Medicine, 2nd edition. Little, Brown and Company, Boston, 1992a pp. 3-6.

Rom WN. Benign pneumoconioses. In: Rom WN, ed. Environmental and Occupational Medicine, 2nd edition. Little, Brown and Company, Boston, 1992b, pp. 479-88.

Rom WN, Crystal RG. Consequences of chronic inorganic dust exposure. In: Crystal RG, West JB, Barnes PJ, Cherniack NS, Weibel ER, Eds. The lung. Scientific Foundations. Raven Press, New York, 1991, pp. 1885-97.

Rom WN. Basic mechanisms leading to focal emphysema in coal workers pneumoconiosis. Environ Res 1990;53:16-28.

Rom WN, Basset P, Fells GA, Nukiwa T, Trapnell BC, Crystal RG. Alveolar macrophages release an insulin-like growth factor I-type molecule. J Clin Invest 1988;82:1685-93.

Rom WN, Bitterman PB, Rennard SI, Cantin A, Crystal RG. Characterization of the lower respiratory tract inflammation of nonsmoking individuals with interstitial lung disease associated with chrionic inhalation of inorganic dusts. Am Rev Respir Dis 1987;136:1429-34.

Ross R, Bowen-Pope DF, Raines EW. Platelet-derived growth factor: its potential roles in wound healing, atherosclerosis, neoplasia, and growth and development. Ciba Found Symp 1985;116:98-112.

Ruckley VA, Gauld SJ, Chapman JS, Davis JM, Douglas AN, Fernie JM, Jacobsen M, Lamb D. Emphysema and dust exposure in a group of coal workers. Am Rev Respir Dis 1984;129:528-32.

Sage H, Farin FM, Striker GE, Fisher AB. Granular pneumocytes in primary culture secrete several major components of the extracellular matrix. Biochem 1983;22:2148-55.

Schaberg T, Orzechowski K, Oesterling C, Lode H, Schuppan D. Simultaneous measurement of collagen type IV related antigen and procollagen type-III-N-propeptide levels in bronchoalveolar lavage. Eur Respir J 1994;7:1221-6. 
Schall T], Lewis M, Koller KJ, Lee A, Rice GC, Wong GHW, Gatanaga T, Granger GA, Lentz R, Raab $\mathrm{H}$, Kohr WJ, Goeddel DV. Molecular cloning and expression of a receptor for tumor necrosis factor. Cell 1990;61:361-70.

Schapira RM, Ghio AJ, Effros RM, Mossisey J, Almagro UA, Dawson CA, Hacker AD. Hydroxyl radical production and lung injury in the rat following silica or titanium oxide instillation in vivo. Am J Respir Cell Mol Biol 1995;12:220-6.

Schilderman PAEL, van Maanen JMS, ten Vaarwerk FJ, Lafleur MVM, Westmijze EI, ten Hoor F, Kleinjans JCS. The role of prostaglandin $\mathrm{H}$ synthase-mediated metabolism in the induction of oxidative DNA-damage by BHA metabolites. Carcinogenesis 1993;14:1297-302.

Schins RPF, Derhaag TJJM, de Jong J, Bast A, Borm PJA. Serum total radical trapping anti-oxidant parameter (TRAP) in coal workers. Clin Chem 1994a;40:1470-2.

Schins RPF, Borm PJA, Lenaerts L. Tumor necrosis factor (TNF) and progression of Coal Workers' Pneumoconiosis in retired coal miners. In: Mohr U, Dungworth DL, Mauderly JL, Oberdörster G, Eds. Toxic and Carcinogenic Effects of Solid Particles in the Respiratory Tract. ILSI Press, Washington, 1994b, pp. 423-7.

Schins RPF, Derhaag TJJM, Préat B, Borm PJA. Ex-vivo release of tumor necrosis factor- $\alpha$ from monocytes is a good predictor of pneumoconiosis in ex-coal workers. Am J Respir Crit Care Med 1994c;149:A556.

Schins RPF, Borm PJA. Serum procollagen type III peptide in Coal Workers' pneumoconiosis: A five year follow-up study. Exp Lung Res 1994;20:445-55.

Schins RPF, Schilderman PAEL, Borm PJA. Oxidative DNA-damage in peripheral blood lymphocytes of coal workers. Int Arch Occup Environ Health 1995;67:153-7.

Schins RPF, Borm PJA. Epidemiological evaluation of release of monocyte TNF- $\alpha$ as an exposure and effect marker in pneumoconiosis: a five year follow up study. Occup Environ Med 1995a;52:441-50.

Schins RPF, Borm PJA. Plasma levels of soluble tomour necrosis factor receptors are increased in coal miners with pneumoconiosis. Eur Respir J 1995b;8:1658-63.

Schins RPF, van Hartingsveldt B, Borm PJA. Ex vivo cytokine release from whole blood. A routine method for health effect screening. Exp Toxic Pathol 1996;48:494-6.

Schmidt JA, Mizel SB, Cohen D, Green I. Interleukin-1, a potential regulator of fibroblast proliferation. J Immunol 1982;128:2177-82.

Schmidt JA, Oliver CN, Lepe-Zuniga JL, Green I, Grey I. Silica stimulated monocytes release fibroblast proliferation factors identical to interleukin 1: potential role for interleukin-1 in the pathogenesis of silicosis. J Clin Invest 1984;73:1462-72.

Schollmeier K. Immunologic and pathophysiologic role of tumor necrosis factor. Am J Respir Cell Mol Biol 1990;3:11-2.

Schraufstätter IU, Cochrane CG. Oxidants: Types, sources and mechanisms of injury. In: Crystal RG, West JB, Bames PJ, Chemiack NS, Weibel ER, Eds. The lung. Scientific Foundations. Raven Press, New York, 1991, pp. 1803-10.

Schulte PA. A conceptual framework for the validation and use of biologic markers. Environ Res $1989 ; 48: 129-44$.

Schulte PA. Contribution of biological markers to occupational health. Am J Ind Med 1991;20:43546.

Schulte PA, Rothman N, Schottenfeld D: Design considerations in molecular epidemiology. In: Schulte PA, Perera FP, Eds. Molecular epidemiology. Principles and practices. Academic Press, San Diego, 1993, pp. 159-98.

Schulte PA: A conceptual and historical framework for molecular epidemiology. In: Schulte PA, Perera FP, Eds. Molecular epidemiology. Principles and practices. Academic Press, San Diego, 1993, pp. 3-44.

Schulte PA, Perera FP. Validation. In: Schulte PA, Perera FP, Eds. Molecular epidemiology. Principles and practices. Academic Press, San Diego, 1993, pp. 81-107. 
Schwartz J, Weiss ST. Relationship between dietary vitamin C intake and pulmonary function in the first national health and nutrition examination survey (NHANES I). Am J Clin Nutr 1994; 59: 110-4.

Seaton A, Lamb D, Brown WR, Sclare F, Middleton WG. Pneumoconiosis in oil shale miners. Thorax $1981 a ; 36: 412-8$.

Seaton A, Dodgson J, Dick JA, Jacobsen M. Quartz and pneumoconiosis in coalminers. Lancet 1981;2:1272-5.

Seaton A. Surveillance of work related and occupational respiratory disease - SWORD. Thorax 1991;46:548.

Seaton A, Dodgson J, Dick JA, Jacobsen M. Quartz and pneumoconiosis in coalminers. Lancet 1981;2 (8285): 1272-75.

Seidegard J, Pero RW, Miller DG, Beattie EJ. A glutathione transferase in human leukocytes as a marker for the susceptibility to lung cancer. Carcinogenesis 1986;7:751-53.

Seidegard J, Pero RW, Markowitz MM, Roush G, Miller DG, Beattie EJ. Isoenzyme(s) of glutathione transferase (class $\mathrm{m}$ ) as a marker for the susceptibility to lung cancer: a follow-up study. Carcinogenesis 1990;11:33-6.

Selman M, Pardo A, Barquin N, Sansores R, Ramirez R, Ramos C, Montano M, Stricklin G. collagenase and collagenase inhibitors in bronchoalveolar lavage fluids. Chest 1991;100:151-5.

Seyer JM, Hutcheson ET, Kang AH. Collagen polymorphism in idiopathic chronic pulmonary fibrosis. J Clin Invest 1976;57:1498-507.

Shahzeidi S, Mulier B, de Crombrugghe B, Jeffery PK, McAnulty RJ, Laurent GJ. Increased type I collagen gene expression during bleomycin induced lung fibrosis. Thorax 1993;48:622-8.

Shahzeidi S, Jeffery PK, Laurent GJ, McAnulty RJ. Increased type I procollagen mRNA transcripts in the lungs of mice during the development of bleomycin-induced fibrosis. Eur Respir J 1994;7:1938-43.

Shapiro L, Clark BD, Orencole F, Poutsiaka DD, Granowitz EV, Dinarello CA. Detection of tumor necrosis factor soluble receptor p55 in blood samples from healthy and endotoxemic humans. J Infect Dis 1993; 167: 1344-50.

Shennan DH, Washington JS, Thomas DJ, Dick YA, Kaplan YS, Bennet JG. Factors predisposing to the development of progressive massive fibrosis in coalminers. Br J Ind Med 1981;38:321-6.

Sheperd VL. Cytokine receptors of the lung. Am J Respir Cell Mol Biol 1991;5:403-10.

Shimokado K, Raines EW, Madtes DK, Barrett TB, Benditt EP, Ross R. A significant part of macrophage-derived growth factor consists of at least two forms of PDGF. Cell 1985;43:277-86.

Shull S, Heintz NH, Periasamy M, Manohar M, Janssen YM, Marsh JP, Mossman BT. Differential regulation of antioxidant enzymes in response to oxidants. J Biol Chem 1991;266:24398-403.

Silicosis and Silicate Disease Committee. Diseases associated with exposure to silica and nonfibrous silicate materials. Arch Pathol Lab Med 1988;112:673.

Sims JE, March CJ,Cosamn D, Widmer MB, MacDonald HR, McMahan CJ, Grubin CE, Wignall JM, et al. CDNA expression clonong of the IL-1 receptor, a member of the immunoglobulin family. Science 1988:241:585-9.

Sjöstrand $M$. Cell reactions in the development of lung fibrosis. An animal inhalation model. 1989, Thesis, Kompendietryckeriet-Källered, pp. 187.

Sluys-Cremer GK, Walters LG, Sichel HS. Chronic bronchitis in miners and non-miners: an epidemiological survey of community in the gold-mining area in the Transvaal. Br J Ind Med 1967;24:1-12.

Sinith CM, Kelsey KT, Wiencke JK, Leyden K, Levin S, Christiani DC. Inherited glutatione-Stransferase deficiency is a risk factor for pulmonary asbestosis. Cancer Epidemiol Biomarkers Prev 1994:3:471-7.

Smith GA, Davis T, Anderson D, Solam L, Beckmann MP, Jerzy R, Dower SK, Cowman D, Goodwin RG. A receptor for tumor necrosis factor defines an unusual family of cellular and viral proteins. Science 1990;248:1019-23.

Smith RE, Strieter RM, Zhang K, Phan SH, Standiford TJ, Lukacs NW, Kunkel SL. A role for C-C chemokines in fibrotic lung disease. J Leukoc Biol 1995;57:782-7. 
Snyder GL: Interstitial lung disease: pathogenesis, pathophysiology, and clinical presentation. In: Schwarz MI, King Jr TE, Eds. Interstitial lung disease. BC Decker Inc., Toronto, 1988, pp. 1-14.

Snyderman R, Goetzl EJ. Molecular and cellular mechanisms of leukocyte chemotaxis. Science 1981;213:830-7.

Soutar C, MacLaren W, Annis R, Melville A: Quantitative relations between exposiure to respirable coal mine dust and coalworkers' simple pneumoconiosis in men who have worked as miners but have left the coal industry. $\mathrm{Br}$ J Ind Med 1986;43:29-36.

Soutar CA. Update on lung disease in coal workers. Br J Ind Med 1987;44:145-8.

Soutar and Hurley JF. Relation between dust exposure and lung function in miners and ex-miners. $\mathrm{Br}$ ) Ind Med 1986;43:307-20.

Spinas GA, Keller U, Brockhaus M. Release of soluble receptors fo tumor necrosis factor (TNF) in relation to circulating TNF during experimental endotoxinemia. J Clin Invest 1992;90:533-6.

Sporn MB, Roberts $A B$. The transforming growth factor-betas: past, present, and future. Ann NY Acad Sci 1990;593:1-6.

Sporn MB, Roberts AB, Wakefield LM, Assoian RK. Transforming growth factor-beta: biological function and chemical structure. Science 1986;233:532-4.

Spurzem JR, Saltini C, Rom WN, Winchester RJ, Crystal RG. Mechanisms of macrophage accumulation in the lungs of asbestos-exposed subjects. Am Rev Respir Dis 1987;136:276-80.

Stockley RA. Proteolytic enzymes, teir inhibitors and lung diseases. Clin Sci 1983;64:119-26.

Stookey LL. Ferrozine - a new spectrophotometric reagent for iron. Anal Chem 1970;42:779-81.

Swaen GMH, Meijers JMM, Slangen JJM. Risk of gastric cancer in pneumoconiotic coal miners and the effect of respiratory impairment. Occup Environ Med 1995;52:606-10.

Sung SS, Bjorndahl JM, Wang CY, Kao HT, Fu SM. Production of tumor necrosis fctor/cachectin by human $\mathrm{T}$ cell lines and peripheral blood $\mathrm{T}$ lymphocytes stimulated by phorbol myristate acetate and anti-CD3 antibody. J Exp Med 1988;167:937-53.

Swartz S, Konishi F, Sanjabi P. Dietary assessment of coal miners in southern Illinois. Am J Clin Nutr 1978;31:1465-8.

Takemura T, Rom WN, Ferrans VJ, Crystal RG. Morphologic characterization of alveolar macrophages from subjects with occupational exposure to inorganic particles. Am Rev Respir Dis $1989 ; 140: 1674-85$.

Takeuchi T, Morimoto K. Crocidolite asbestos increased 8-hydroxydeaxygruanosine levels in cellular DNA of a human promyelocytic leukemia cell line, HL60. Carcinogenesis 1994;15:63539.

Tanner GW, Welbom BM, Sheperd VL. Tumor necrosis factor- $\alpha$ and interleukin-1 $\alpha$ synergistically enhance phorbol myristate acetate-induced superoxide production by rat bone marrow-derived macrophages. Am J Respir Cell Mol Biol 1992;7:379-84.

Thomas ED, Ramberg RE, Sale GE, Sparkes RS, Golde DW. Direct evidence for a bone marrow origin of the alveolar macrophage in man. Science 1976;192:1016-8.

Tilg H, Vogel W, Wiedermann CJ, Shapiro L, Herold M, Judmaire G, Dinarello. Circulating interleukin-1 and tumor necrosis factor antagonisis in liver disease. Hepatol 1993;18:1132-38.

Toth KM, Clifford DP, Berger EM, White CW, Repine JE. Intact human erythrocytes prevent hydrogen peroxide-mediated damage to isolated perfused rat lungs and cultured bovine pulmonary artery endothelial cells. J Clin Invest 1984;74:292-5.

Tourmann JL, Kaufmann R. Biopersistence of the mineral matter of coal mine dusts in silicotic human lungs: Is there a preferential release of iron? Environ Health Perpect 1994;102:265-8.

Tracey KJ, Fong Y, Hesse DG, Manogue KR, Lee AT, Kuo GC, Lowry SF, Cerami A. Anticachectin/TNF monoclonal antibodies prevent septic shock during lethal bacteraemia. Nature 1987;330:662-4

Trush MA, Kensler TW. An overview of the relationship between oxidative stress and chemical carcinogenesis. Free Rad Biol Med 1991;10:201-10.

Ulich TR, Yin S, Remick DG, Russell D, Eisenberg SP, Kohno T. Intratracheal administration of endotoxin and cytokines. IV. The soluble tumor necrosis factor receptor type I inhibits acute inflammation. Am J Pathol 1993;142:1335-8. 
Vallyathan V, Craighead JE. Pulmonary pathology in workers exposed to nonasbestiform talc. Hum Pathol 1981;12:28-35.

Vallyathan V, Shi XL, Dalal NS, Irr W, Castranova V. Generation of free radicals from freshly fractured silica dust. Putential role in acute silica-induced lung injury. Am Rev Respir Dis 1988:138:1213-9.

Vallyathan V, Lap'p L, Weber SL, Shumaker J, Leonard S, Pack D, Castranova V. Evidence for the increased oxidant burden and upregulation of antioxidants in asymptomatic coal workers' lung lavage fluid. Am J Respir Crit Care Med 1995:151:A711.

Van Asbeck BS, Hoidal J, Vercellotti GM, Schwartz BA, Moldow CF, Jacob HS. Protection against lethal hyperoxia by tracheal insufflation of erythrocytes: the role of red cell glutathione. Science 1985;227:756-9.

Van der Schouw YT, Segers MFG, Smits L, Thomas CMG, Verbeek ALM, Wobbes Th. Towards a more standardized assessment of diagnostic tumour markers. Int J Oncol 1993;3:979-85.

Vanhée D, Gosset P, Marquette CH, Wallaert B, Lafitte JJ, Gosselin B, Voisin C, Tonnel AB. Secretion and mRNA expression of TNF $\alpha$ and IL-6 in the lung of pneumoconiotic patients. Am J Respir Dis 1993;147:A906.

Vanhée D, Gosset P, Marquette CH, Wallaert B, Lafitte JJ, Gosselin B, Voisin C, Tonnel AB. Secretion and mRNA expression of TNF $\alpha$ and IL- 6 in the lungs of pneumoconiotic patients. Am J Respir Crit Care Med 1995;152:298-306.

Vanhée D, Gosset P, Wallaert B, , Voisin C, Tonnel AB. Mechanisms of fibrosis in coal workers pneumoconiosis. Increased production of platelet-derived growth factor, insulin-like growth factor type $\mathrm{I}$, and transforming growth factor $\beta$ and relationship to disease severity. Am I Respir Crit Car Med 1994;150:1049-55.

Van Sprundel MPM. Pneumoconioses: The situation in developing countries. Exp Lung Res 1990;16:513.

Vermeire P. Contribution of HRCT-scan to the radiologic diagnosis of anthraco-silicosis. In: Coalworkers' Occupational disease Project. Medical workshop, Hasselt 1-10 sept 1993, Belgium. Book of Abstracts, 1993.

Vogelmeier C, Hubbard RC, Fells GA, Schnebli HP, Thompson RC, Fritz H, Crystal RG. Antineutrophil elastase defense of the normal human respiratory epithelial surface provided by the secretory leukoprotease inhibitor. J Clin Invest 1991;87:482-8.

Voisin C, Wallaert B, Aerts C, Grosbois JM. Broncho-alveolar lavage in coal worker's pneumoconiosis. Oxidant and anti-oxidant activities of alveolar macrophages. In: Beck EG and Bignon J, Eds. In vitro effects of mineral dusts. NATO ASI series, vol G3, Springer-Verlag Berlin, 1985 pp. 93-100.

Vu VT. Assessment of the potential health effects of natural and man-made fibers and their testing needs: Perspectives of the US Environmental Protection Agency. In: Mohr U, Dungworth DL, Mauderly JL, Oberdörster G, Eds. Toxic and Carcinogenic Effects of Solid Particles in the Respiratory Tract . ILSI Press, Washington, 1994, pp. 368-84.

Wagner JC, Sleggs CA, Marchand P. Diffuse pleural mesothelioma and asbestos exposure in the North Western Cape Province. Br J Ind Med 1960;17:260-71.

Wahl SM, Hunt DA, Wakefield LM, McCartney-Francis N, Wahl LM, Roberts AB, Sporn MB. Transforming growth factor type beta induces monocyte chemotaxis and growth factor production. Proc Natl Acad Sci USA 1987;84:5788-92.

Wakefield LM, Smith DM, Masui T, Harris CC, Sporn MB. Distribution and modulation of the cellular receptor for transforming growth factor-beta. J Cell Biol 1987;105:965-75.

Walker C, Bauer W, Braun RK, Menz G, Braun P, Schwarz F, Hansel TT, Villiger B. Activated T cells and cytokines in bronchoalveolar lavage from patients with various lung diseases associated with eosinophilia. Am J Respir Crit Care Med 1994;150:1038-48.

Wallaert B, Lasallae P, Fortin F, Aerts C, Bart F, Foumier E, Voisin C. Superoxide dismutase anion generation by alveolar inflammatory cells in simple pneumoconiosis and in progressive massive fibrosis of nonsmoking coal workers. Am Rev Respir Dis 1990;141:129-33. 
Warren JS, Kunkel ST, Cunningham TW, Johnson KJ, Ward PA. Macrophage-derrived cytokines amplify immune complex-triggered $\mathrm{O} 2-\bullet$ responses by rat alveolar macrophages. Am I Pathol 1988;130:489-95.

Watson AJ, Black J, Doigt AT, Nagelschmidt G. Pneumoconiosis in carbon electrode workers. Br J Ind Med 1959;61:274-85.

Wayner DDM, Burton GW, Ingold KU, Barclay LRC, Locke SJ. The relative contributions of vitamin E, urate, ascorbate and proteins to the total peroxyl radical-trapping antioxidant activity of human blood plasma. Biochim Biophys Acta 1987;924:408-19.

Weill $\mathrm{H}$. Future of research into occupational lung disease. $\mathrm{Br}$ J Ind Med 1993;50:481-3.

Weiss SJ. Tissue destruction by neutrophils. N Engl J Med 1989:320:365-76.

Weitzman SA, Graceffa P. Asbestos catalyzes hydroxyl and superoxide radical generation from hydrogen peroxide. Arch Biochem Biophys 1984;228:373-376.

Welgus HG, Campbell EJ, Bar-Shavit Z, Senior RM, Teitelbaum SL. Human alveolar macrophages produce a fibroblast-like collagenase and collagenase inhibitor. J Clin Invest 1985;76:219-24.

Wells IP, Bhatt CV, Falangan M. Kaolinosis: A radiological review. Clin Radiol 1985;36:579-82.

Wewers MD, Herzyk DJ. Alveolar macrophages differ fromblood monocytes in human IL-1 beta release. Quantitation by enzyme-linked immunoassay. J Immunol 1989;143:1635-41.

Wilson JW, Djukanovic R, Howarth PH, Holgate ST. Lymphocyte activation in bronchoalveolar lavage and peripheral blood in atopic asthma. Am Rev Respir Dis 1992;145:958-60.

Wolpe SD, Davatelis G. Sherry B, Beutler B, Hesse DG, Nguyen HT, Moldawer LL, Nathan CF, Lowry SF, Cerami A. Macrophages secrete a novel heparin-binding protein with inflammatory and neutrophil chemokinetic properties. J Exp Med 1988;167:570-81.

Wong GH, Goeddel DV. Induction of manganous superoxide dismutase by tumor necrusis factor: possible protecitve mechanism. Science 1988;242:941-4.

Wouters EFM, Jorna THJM, Westenend M. Respiratory effects of coal dust exposure: Clinical effects and diagnosis. Exp Lung Res 1994; 20: 385-94.

Yamauchi K, Martinet X, Basset P, Fells GA, Crystal RG. High levels of transforming growth factor-beta are present in the epithelial lining fluid of the normal human lower respiratory tract. Am Rev Respir Dis 1988;137:1360-3.

Zalma R, Bonneau L, Jaurand MC, Guignard J, Pezerat H. Formation of oxy-radicals by oxygen reduction arising from the surface activity of asbestos. Can J Chem 1987;65:23.38-41.

Zedda $S$, Aresini $G$, Ghezzi $I$, et al., Lung function in relation to radiographic changes in asbestos workers. Respiration 1973;30:13-140.

Zhang Y, Lee TC, Guillemin B, Yu MC, Rom WN. Enhanced IL-1 beta and tumor necrosis factoralpha rlease and messenger RNA expression in macrophages from idiopathic pulmonary fibrosis or after asbestos exposure. J Immunol 1993;150:4188-96.

Zielhuis RL, Henderson P Th. Definitions of monitoring activities and their relevance for the practice of occupational health. Int Arch Occup Environ Health 1986;57:249-57.

Ziskind M, Jones RN, Weill H. Silicosis. Am Rev Respir Dis 1976;113:643-65.

Zitnik RJ, Zheng T, Elias JA. CAMP inhibition of interleukin-1-induced interleukin- 6 production by human lung fibroblasts. Am J Physiol 1993;264:L253-60. 

Elk meetbaar biologisch effect tussen blootstelling en een daaruit resulterende ziekte kan men als "biomarker" bestempelen. Dit proefschrift beschrijft de evaluatie van een aantal biomarkers in relatie tot blootstelling aan mineraal stot. Aan de hand van een 5-jaar follow-up van mijnwerkers (Kempense Steenkoolmijnen, België) die oorspronkelijk bij diverse dwarsdoorsnede-studies betrokken zijn geweest, is onderzocht in hoeverre deze parameters in het bloed relevant zijn voor mensen die (beroepsmatig) zijn blootgesteld aan mineraal stof en derhalve mogelijk een verhoogd risico lopen op het onstaan dan wel voortschrijden van aan deze blootstelling gerelateerde ziekten.

In hoofdstuk 1 wordt een kort overzicht gegeven van de voornaamste soorten longaandoeningen die kunnen onstaan bij blootstelling aan bekende en veel voorkomende vormen van mineraal stof zoals asbest, silica en mijnstof. Naast stof-specifieke ziektebeelden (bijvoorbeeld mesothelioom door asbest) veroorzaken diverse soorten mineraal stof ook min of meer gemeenschappelijke gezondheidseffecten waaronder longfunctie-verlies en longfibrose. Tot de voornaamste gezondheidseffecten die kunnen optreden na inhalatie van mijnstof (samengevat in hoofdstuk 2) behoren mijnwerkerpneumoconiose (MWP), chronische bronchitis, vermindering van longfunctie en emphyscem. De vraag of (silica bevattend) mijnstof al dan niet (long)kanker kan veroorzaken is vooralsnog niet opgehelderd en is momenteel (oktober '96) underwerp van een IARC-werkgroep. MWP wordt gediagnostiseerd door beroepsanamnese en een thoraxfoto aan de hand van standaard criteria vastgesteld door het "International Labour Office" (ILO), waarbij MWP in diverse stadia geclassificeerd wordt op grond van aantal en grootte van opaciteiten. In tegenstelling tot progressieve massieve fibrose (PMF) is er bij milde vormen van MWP slechts sprake van geringe longfibrose. Het onstaan van longfibrose uit zich in een excessieve toename in depositie van collagenen.

De biologische mechanismen die ten grondslag liggen aan de door mijnstof geïnduceerde aandoeningen worden beschreven in hoofdstuk 3 aan de hand van in vitro en in vivo studies. Veelal wordt naast de effecten van mijnstof ook verwezen naar die van silica en asbest. Diverse soorten mineraal stof genereren aan de hand van directe (oppervlakte) eigenschappen van het stof dan wel via activatie van fagocyterende cellen (macrofagen, neutrofielen) reactieve zuurstofspecies (RZS). Wanneer de vorming van RZS dermate groot is dat antioxydatieve afweermechanismen waarover de mens beschikt niet afdoende zijn, kan oxydatieve schade optreden aan onder meer eiwitten, celmembranen (lipidperoxydatie) en DNA. Elke verandering in activiteiten of concentraties van antioxydanten kan worden beschouwd als indicatie voor deze zogenaamde "oxydatieve stress". Naast RZS produceren de door stof geactiveerde fagocyten (maar ook andere celtypen zoals alveolair epitheel) onder meer cytokines en groeifactoren die op hun beurt weer in staat zijn om secundaire cellen te 
activeren en aan te zetten tot productie van diverse factoren (o.a. cytokines, RZS). Zo blijkt bijvoorbeeld dat mineraal stof macrofagen in vitro kan aanzetten tot de "release" van TNF. Dit cytokine blijkt in staat om in vitro de deling van fibroblasten te stimuleren en blijkt bij proefdieren het onstaan van fibrose door silica aanzienlijk te versterken; anderzijds wordt in aanwezigheid van TNFantilichamen in het zelfde model silicose grotendeels voorkomen. RZS en diverse cytokines worden in verband gebracht met het onstaan van fibrose alsook andere longaandoeningen (emphyseem, kanker). Longfibrose onstaat als gevolg van verhoogde depositie van met name door fibroblasten geproduceerde type III collagenen (in vroege lesies) en type I collagenen. De in dit proefschrift beschreven studies zijn opgesplitst in drie delen waarin achtereenvolgens markers van oxydatieve stress, cytokine release en collageen depositie zijn bestudeerd in relatie tot longaandoeningen bij mijnwerkers.

Het onderzoeksdesign (beschreven in hoofdstuk 4) is als volgt: 156 mijnwerkers (Kempense Steenkoolmijnen, België) participerend in drie studies uit 1987, zijn opgevolgd met het doel om de bij deze studies onderzochte parameters prospectief te relateren aan het onstaan dan wel het voortschrijden van MWP. Dit is gebeurd op grond van gepaarde analyse van thoraxfoto's over cen tijdsinterval van vijf jaar. Aan het eind van de follow-up zijn de betreffende markers opnieuw bepaald, en zijn tevens nieuwe markers bestudeerd (transversale en retrospectieve analyses). Om een mogelijke verband tussen de diverse parameters met blootstelling te bestuderen is de cumulatieve blootstelling aan respirabel mijnstof geschat aan de hand van "job-exposure" matrices, en is een controle groep van niet beroepsmatig aan (mineraal) stof blootgestelde personen meegenomen. Obstructieve longaandoeningen zijn door middel van spirometrie, impendatiemeting en vragenlijsten in kaart gebracht. In deelgroepen zijn additionele analyses uitgevoerd waaronder HRCT-scans en bepalingen van diffusiecapaciteit, en is retrospectief longfunctiedaling berekend over een periode van 10 jaar voorafgaand aan de oorspronkelijke studies.

Uit de resultaten van de follow-up $(n=104)$ blijkt dat ondanks dat alle mijnwerkers niet meer actief zijn (vanwege algehele mijnsluiting in België) bij een aanzienlijk aantal personen progressie (45\%) en in enkele gevallen zelfs nieuwvorming van MWP voor te komen (5\%) (hoofdstukken 8 en 11). Schatting van de cumulatieve stofblootstelling toont een viervoudige variatie van blootstelling aan ten op zichte van het aantal jaren ondergronds (hoofdstuk 12), en blijkt gerelateerd aan de score van de thoraxfoto's, aan de HRCT-scans (hoofdstuk 12), én aan de progressie van MWP (hoofdstuk 8).

In hoofdstukken $5 \mathrm{t} / \mathrm{m} 7$ worden de oxydative DNA schade en antioxydatieve status besproken. Aan het eind van de follow-up is de antioxydatieve capaciteit van serum bepaald aan de hand van de TRAP-assay (hoofdstuk 5). Deze blijkt significant verhoogd te zijn bij mijnwerkers die gedurende de vijfjarige periode voorafgaand aan de bloedafname MWP ontwikkelden, zowel ten opzichte van 
mijnwerkers zonder MWP als ten opzichte van mijnwerkers met reeds aanwezige MWP. Als mat voor oxydatieve DNA schade is in het DNA van lymfocyten de hoeveelheid 7-hydro-8-oxo-2'deoxyguanosine bepaald (hoofdstuk 6). De schade blijkt niet te verschillen tussen mijnwerkers met of zonder MWP; deze is echter wel verhoogd bij mijnwerkers ten opzichte van niet-blootgestelde controles. De verhoogde oxydatieve schade in de lymfocyten van de mijnwerkers staat in mogelijk verband met de door anderen aangetoonde aanwezigheid van stabiele radicalen (en meer specifiek in de lymfeklieren) in longbiopten van (ex) mijnwerkers. In hoofdstuk 7 zijn diverse antioxidanten gerelateerd aan prospectieve veranderingen in MWP, aan longfunctiedalingen (retrospectief), en aan chronische bronchitis. Tevens wordt in dit hoofdstuk de relevantie van de gecombineerde analyse van diverse antioxydant parameters in relatie tot pneumoconiose alsmede obstructieve longaandoeningen besproken. Voornaamste bevindigen zijn een verhoogde SOD-activiteit in erythrocyten van de personen die vervolgens MWP ontwikkelden, en een verlaagde GSH concentratie in chronische bronchitis. Aangezien zowel in vitro als in vivo is aangetoond dat verhoogde MnSOD expressie een indicator van oxydatieve stress is, zou men de verhoogde SOD-waarden in erythrocyten van personen met progressie van MWP kunnen verklaren als een weerspiegeling van verhoogde pathogene activiteit van stof in de long. Een verlaagde GSH acitiviteit in bronchoalveolaire lavage bij obstructieve longaandoeningen is door andere onderzoekers beschreven. In algemene zin tonen de in dit deel van het proefschrift beschreven resultaten aan dat oxydatieve stress, alsmede de hiermee geassocieerde oxydatieve DNA schade in personen blootgesteld aan mineraal stof in verhoogde mate aanwezig zijn buiten de long c.q. in perifeer bloed, én zelfs aanwezig blijven ondanks een (jarenlange) afwezigheid van deze (beroepsmatige) blootstelling.

De hoofdstukken $8 \mathrm{t} / \mathrm{m} 10$ behandelen achtereenvolgens de ex vivo release van TNF door perifere bloed monocyten (h8), de plasma concentraties van de $55 \mathrm{kD}$ en $75 \mathrm{kD}$ receptoren van TNF (h9), en de gecombineerde evaluatie van TNF, TGF $\beta$ en IL-6 (h10). In overeenstemming met de rol van TNF in silicose bij de muis, indiceren de resultaten dat dit cytokine ook een cruciale factor is bij fibrose geinduceerd door mijnstof. De herhaalde dwarsdoorsnede analyse toont aan dat release van TNF na ex vivo stimulatie van monocyten door mijnstof zowel in actieve als in ex-mijnwerkers verhoogd is ten opzichte van de niet blootgestelde controles, en laat tevens zien dat deze release in beide studies een afnemende trend vertoont met een toenemende gradatie van MWP. Voorts blijkt uit prospectieve analyse dat zowel een verhoogde TNF release, alsook de cumulative stofblootstelling de progressie van MWP verklaren. Stofblootstelling en TNF release zijn echter niet aan elkaar gecorreleerd. De gestimuleerde TNF release op beide meetpunten is duidelijk gecorreleerd, echter met name in de mijnwerkers zonder progressie, hetgeen indirect wijst op een invloed van het 
ziekteproces op de release van TNF door monocyten. De relatief stabiele fenotypische variaties in TNF release berusten mogelijk op in het kader van andere aandoeningen beschreven polymorfismen van het TNF-promotor gen. In tegenstelling tot de gestimuleerde TNF release blijkt de spontane TNF release verlaagd te zijn in de ex-mijnwerkers ten opzichte van de actieve mijnwerkers. Waarschijnlijk wordt deze spontane release met name door "actuele" blootstelling bepaald, terwijl de gestimuleerde release gekoppeld is aan blootstellingsverleden, al dan niet bepaald door een voortdurende reactie in de long op geaccumuleerd mijnstof. Daarentegen blijkt de release van TGF $\beta$ zowel spontaan als na ex vivo stimulatie verhoogd te zijn in de ex-mijnwerkers, en in beide gevallen verhoogd ten opzichte van de nooit blootgestelden. IL-6 release vertoont overeenkomsten met die van TNF, met uitzondering van de door silica gestimuleerde IL-6 release die nagenoeg gelijk blijkt te zijn aan de baseline release van IL-6. De ex vivo gestimuleerde release van TGF $\beta$ en TNF is positief gecorreleerd in niet blootgestelde controles, maar negatief in de mijnwerkers met MWP, hetgeen wijst op de mogelijk tegengestelde rol van beide cytokines in de pathogenese van MWP. Tevens zijn bij de mijnwerkers ook verhoogde plasma concentraties van de TNF receptoren (sTNF-R55 en -R75) gevonden ten opzichte van de niet blootgestelde controles, en blijkt dat medicijngebruik ten tijde van bloedafname een verhogend effect op sTNF-R55 concentraties heeft. Prospectief onderzoek is echter nodig om te bepalen in hoeverre de sTNF-R's, TGF $\beta$ en IL-6 in navolging van TNF een voorspellende waarde hebben met betrekking tot de progressie van MWP.

In de hoofdstukken 11 en 12 is de rol van serum type III procollageen peptide (PIIIP) bestudeerd. In tegenstelling tot het oorspronkelijke dwarsdoorsnede onderzoek is bij de ex-mijnwerkers geen verband tussen PIIIP en thorax of HRCT score aanwezig, en blijkt PIIIP niet gerelateerd aan de longfunctie (spirometrie, impedantiemeting, diffusiecapaciteit) (hoofdstuk 12). Bovendien blijkt er geen verband met enige parameter van blootstelling te zijn, uitgezonderd een negatieve correlatie tussen cumulatieve stofblootstelling en PIIIP in personen met MWP. De resultaten staan in contrast met andere studies waarbij PIIIP gerelateerd bleek te zijn aan asbestblootstelling en aan de ernst van sarcoidose. Longitudinale analyse toont aan dat serum PIIIP meting in MWP geen marker is voor de individuele gevoeligheid voor het ontstaan of de progressie van fibrose (hoofdstuk 11). Nadere studie is noodzakelijk om het precieze verband tusssen serum PIIIP en processen van collageen depostie en afbraak zowel in de "gezonde" long als in MWP beter te begrijpen.

In dit proefschrift zijn diverse markers van blootstelling aan- en effecten van mijnstof bestudeerd en zijn parallel nieuwe inzichten in het ontstaan van longaandoeningen door blootstelling aan mijnstof gegenereerd. De vraag rijst nu in hoeverre de betreffende bevindingen geëxtrapoleerd kunnen worden naar 
effecten van blootstelling aan andere soorten mineraal stof. Op grond van de resultaten beschreven in dit proefschrift en aanwijzingen uit dierexperimenteel en in vitro onderzoek lijkt dit "generaliseren" voor markers als TNF en antioxidant parameters meer op te gaan dan bijvoorbeeld voor PIIIP. Enerzijds dus verdient het aanbeveling om de gepresenteerde bevindingen verder te evalueren voor mijnstof in relatie tot specifieke onderzoeksvragen zoals de voorspellende waarde van de markers in nieuwe mijnwerkers of in patienten met PMF. Anderzijds echter kan ook aan onderzoek worden gedacht in het kader van andere vormen van blootstelling of andere soorten mineraal stof, zoals werknemers in de keramische industrie, of bij blootstelling aan stof tijdens processen als (steen)slijpen en zandstralen, en bijvoorbeeld in de bouw. De in dit proefschrift gepresenteerde studies kunnen hierbij als een soort leidraad dienen waarbij zowel het gepresenteerde raamwerk van biomarkers van longaandoeningen door mineraal stof alsmede de specifiek geëvalueerde markers in te passen zijn. 


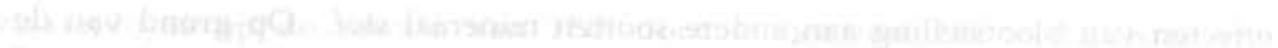

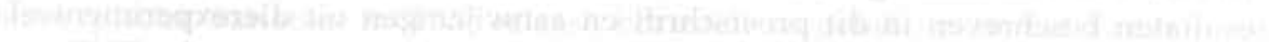

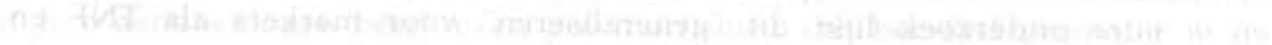

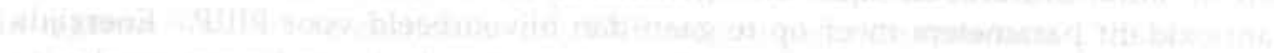

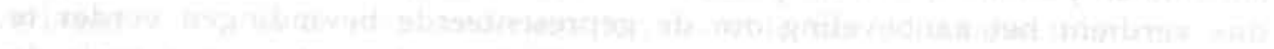

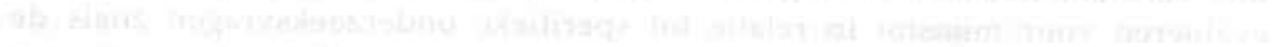

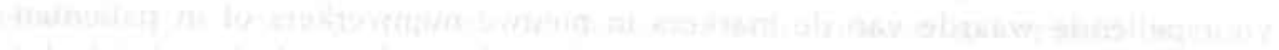

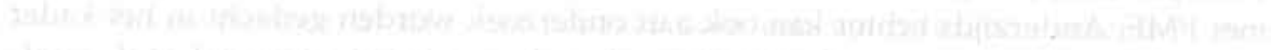

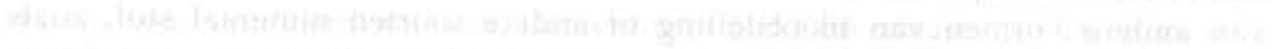

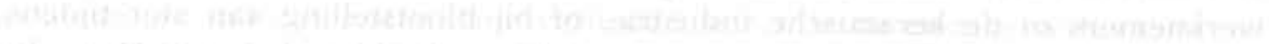

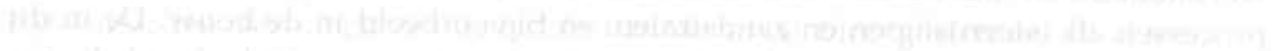

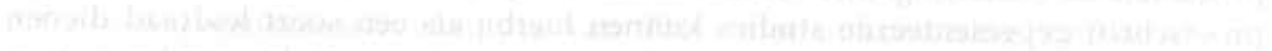

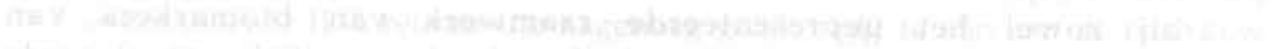

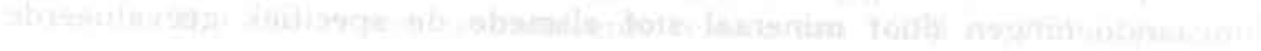




\section{Dankwoord}

Voor velen zal na de titel en mijn naam, dit dankwoord waarschijnlijk zo'n beetje het eerste zijn dat gelezen wordt. Dat is maar goed ook, want men moet weten dat dit boekje weliswaar op mijn naam staat, maar dat vele anderen er zeker op de een of andere manier aan hebben bijgedragen. In de nu volgende dankbetuigingen zou ik "in het vuur van het spel" een enkeling kunnen vergeten. Daarvoor mijn welgemeende excuses.

Op de eerste plaats zou ik mijn co-promotor dr. Paul Borm willen bedanken. Beste Paul, tijdens mijn promonderzoek heb je me op een fantastische manier begeleid. Je introduceerde $\mathrm{mij}$ in het fascinerende gebied van toxicologie van de luchtwegen, en in het bijzonder van stofdeeltjes en vezels: Een bonte en gevariëerde wereld wemelend van 't maagdelijk witte silica tot het roetzwarte mijnstof, en van het zijdezachte katoenstof tot de bikkelharde aramidevezel. Ook wist je me al snel van het belang van de "moleculaire epidemiologie" te overtuigen. Wellicht weet je dus daarom als geen ander welke organoleptische susceptibility marker (of is 't merker?) bepaalt of je die Medoc uit ' 90 na vijf jaar follow-up beter bij een tournedos met bearnaisesaus moet schenken of dat ie vergezeld van wildfond, stoofpeertjes, laurierblad en kruidnagel toch maar rechtstreeks over een haas moet.

Tevens wil ik mijn promotor prof. Jos Kleinjans bedanken. Jos, met name in de laatste fase van het promoveren gaf je mij vele goede tips over hoe nu -zoals dat heet- de publicaties aan elkaar geniet moesten worden en hoe de betreffende niet-strategie het best verantwoord kon worden middels inleiding en discussie. Je vooruitziendheid blijkt onder meer uit het gegeven dat je de aanleiding voor een van mijn stellingen al hebt geschoten lang voordat de wetenschap goed en wel wist wat een 55-kD soluble TNF receptor was, of dat men door een ECD aan een HPLC te hangen uitstekend 7-hydro-8-oxo-2' deoxyguanosine kan meten.

Prof. Miel Wouters, prof. Jos van Engelshoven, prof. Aalt Bast, en dr. Wim Buurman wil ik bedanken voor hun snelle (of zelfs zéér snelle) beourdeling van het manuscript. I am indebted to dr. Paul Schulte for his willingness to participate in the board and his rapid but thorough evaluation of this thesis. Furthermore, I would like to thank him as well as dr. Eileen Kuempel for helpful comments and suggestions.

De Kempense Steenkoolmijnen bedank ik voor de goede ondersteuning gedurende de follow-up. Op de mijnzetel te Zolder, waar het allemaal begon (en 't vuurke op 4 stond) wil ik GEDILO (Hasselt) en in het bijzonder dr. Luc Lenaerts, en Herwig Vinckx danken voor de hulp bij het veldwerk. Tevens wil ik dr. Luc Mariën (GEDILO) en dr. Marc van Sprundel (Universiteit van Antwerpen), en nogmaals dr. Luc Lenaerts noemen vanwege hun volharding tijdens de vermoeiende leessessies van de thoraxfoto's. 
Ir. Bernard Préat dank ik voor de berekening van de cumulatieve stofblootstelling: een onmishaar onderdeel bij de evaluatie van biomarkers !!!! Een bijzonder woord van dank ook aan hen die bij de oorspronkelijke dwarsdoorsnede studies verbonden waren, waarbij ik elk geval John Engelen, Nicole Palmen, Gerard Swaen, Yvonne Janssen en Chris Evelo zou willen noemen. Immers, waar geen spoor is kun je ook niet op een rijdende trein stappen.

Voor hun ondersteuning en tips m.b.t. het cytokine gedeelte wil ik het lab Algemene Heelkunde bedanken, in het bijzonder Mieke Dentener, Gaby Francot, Trudy Jeunhomme, en -nogmaals- Wim Buurman. Je voudrais aussi remercier prof. Bénoit Wallaert et dr. Philippe Gosset pour leur participation et conseils à l'égard du sujét de "TGF". Paulien Schilderman, Jan Lutgerink, Jolanda van Golde en Angelique VanKan dank ik voor hun bijdrage aan het 8-oxo werk. Rob Lamers (radiodiagnostiek, AZM) dank ik voor de plezierige samenwerking met betrekking tot de HRCT-scans, en Tim Jorna en ("perfect") Soedjajadi Keman voor het longfunctie werk. Een bijzonder woord van dank ook aan Thim Derhaag: Thim, bedankt voor alle hulp, met name tijdens de eerste fase van het onderzoek, je doortrapte analyses, je flitstende rijstijl, en natuurlijk je onnavolgbare jongleertruc met de Vica-4 (wat 'n vlugge, ik zag niet eens uw hand bewegen). Voor de statistische hulp, dank ik Fons Kessels van de Vakgroep Epidemiologie. Anke Wijnen (GRAT) wil ik danken vanwege haar hulp bij de terugrapportage van onderzoeksresultaten aan alle participerende mijnwerkers.

Een groot aantal personen kan ik (omwille van privacy) niet bij naam noemen, terwijl dit huekje eigenlijk alleen dankzij hun bijdrage geschreven kon worden. Daarom wil ik hier in het bijzonder alle ex-mijnwerkers van de KS nogmaals van harte bedanken voor hun deelname aan het onderzoek: Gluck Auf!!

()mdat ik -echt waar-geen enkele dag met tegenzin naar 't werk ben gekomen, d.unk ik alle "kompels" van de "UM f.k.a. RL", in het bijzonder alle (ex) medewerkers en gasten van de vakgroep GRAT en (in het prille begin) alle exAMT-ers. Omdat er geen betere plaats is om over elkaars (of andermans) werkzaamheden te roddelen, wil ik toch nog even mijn achtereenvolgende kamergenoten IJmert, Tim en Ivo ("there is no tox like Pernod-tox") noemen.

Tenslotte, omdat ik geen enkele dag met tegenzin van 't werk ging, bedank ik mijn vrienden en familie, en (chipohaia) Yvonne!! 
Zoals velen van mijn jeugdvrienden en vriendinnen werd ik, "inge richtieje Kirchröatscher jóng" geboren in "de vroedvrouwenschool"... te Heerlen, en wel op 27 juli 1966, in de tijd dat vanuit het verre Den Haag werd besloten dat in het gevecht tussen de gasbel en het zwarte goud de laatste maar eens het onderspit zou moeten delven. Overigens kan ik mij van het rijke mijnverleden nauwelijks meer iets herinneren dan het opdoemen van de zwarte berg van cle Willem Sophia wanneer je tijdens een partijtje voetbal op het Carboonplein een afzwaaiende bal achterna holde, en natuurlijk het enorme rangeertraject van het station Spekholzerheide (Utrecht-CS was er niets bij). Na het doorlopen van het Heilig Hart en later d'r Durpel besloot ik naar het Katholiek Gymnasium Rolduc te gaan, precies daar waar wat schoffelwerk van enkele kloosterlingen omstreeks de $12 \mathrm{e}-13 \mathrm{e}$ eeuw er uiteindelijk toe geleid had dat Kerkrade zich (al dan niet terecht) Europa's oudste Steenkoolmijnstad noemt, en -wellicht daarom- op het stadswapen de heilige Lambertus gemerkt is door twee "Pikkele". Na het ondertekenen van het gymnasium-B diploma in 1984, besloot ik Biologie te gaan studeren aan de Katholieke Universiteit Nijmegen waarmee ik, naar naderhand bleek, duidelijk een verkeerde ader had aangeboord. Na een bonte afwisseling van tijdelijk werk en tijdelijke werkloosheid startte ik 1987 aan de Rijksuniversiteit Limburg de studie Gezondheidswetenschappen, waar ik na een jaar dan (toch weer) voor Biologische Gezondheidkunde koos. Na onder meer een stage bij het Laboratoire de Pathologie Cellulaire et Moléculaire van het Institut National de la Santé et de la Recherche Médical (INSERM) te Créteil in Frankrijk, studeerde ik in maart 1992 af als Gezondheidswetenschapper. Ik kon direct van start als onderzoeksassistent bij achtereenvolgens de vakgroep Arbeidsgeneeskunde, Milieugezondheidkunde en Toxicologie (AMT) en de vakgroep Gezondheidsrisico Analyse en Toxicologie (GRAT) van de Rijksuniversiteit Limburg (/Universiteit Maastricht), in een derdegeldstroomproject gesponsord door de -inmiddels ter ziele- Europese Gemeenschap voor Kolen en Staal (EGKS). Met ingang van 1 januari 1993 werd mijn functie geherwaardeerd tot toegevoegd onderzoeker. 


\section{Sally mala inas}

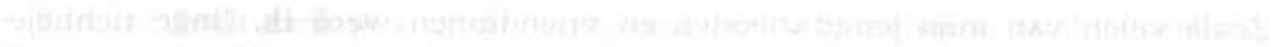

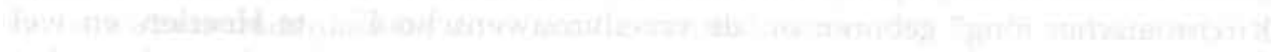

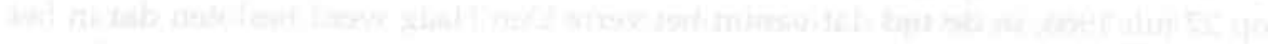

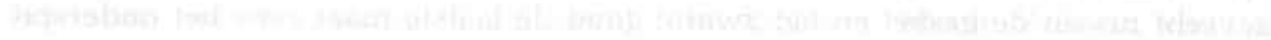

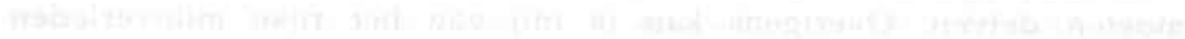

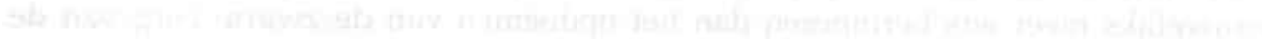

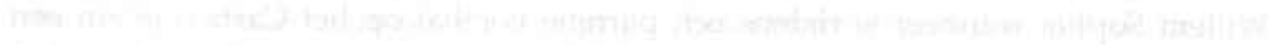

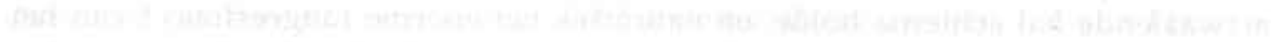

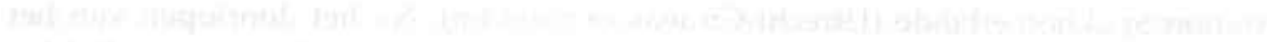

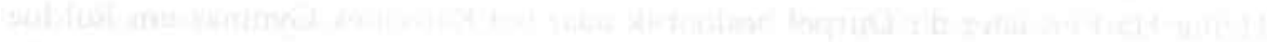

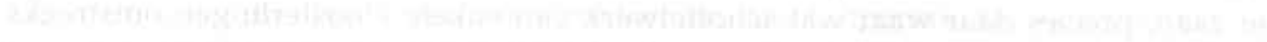

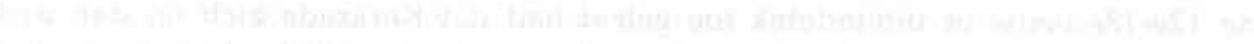

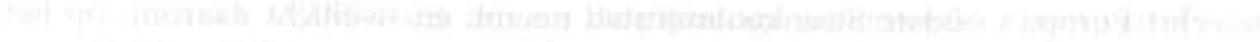

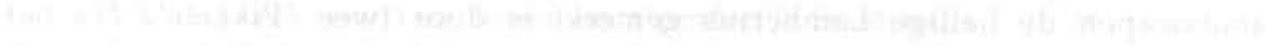

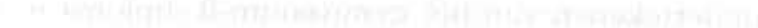

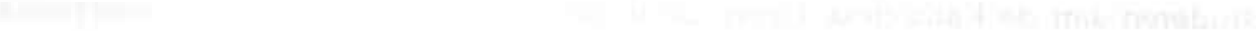




\section{List of publications}

\section{Papers}

Schins RPF, Gosset Ph, Wallaert B, Borm PJA. Multiple cytokines as biomarkers in coal dust exposure and pneumoconiosis: Tumor Necrosis Factor- $\alpha$, Interleukin-6, and Transforming Growth Factor- $\beta$, submitted.

Keman S, Willemse B, Guevarra L, Tollerud DJ, Guevarra L, Schins RPF, Borm PJA. Blood cytokines as markers of respiratory impairment in chemical workers, submitted.

Schins RPF, Keman S, Borm PJA. Blood antioxidant status in coal dust induced respiratory disorders: a longitudinal multi-marker evaluation. Biomarkers, in press.

Schins RPF, van Hartingsveldt B, Borm PJA. Ex vivo cytokine release from whole blood. A routine method for health effect screening. Exp Toxicol Pathol, 1996;48:494-6.

Borm PJA, Schins RPF, Derhaag TJJM, Kant IJ. Cross-shift changes in blood inflammatory markers occur in the absence of airway obstruction in workers exposed to grain dust. Chest 1996; 109:107885.

Schins RPF, Borm PJA. Plasma levels of the soluble tumour necrosis factor (TNF) receptors are increased in coal miners with pneumoconiosis. Eur Respir J 1995; 8: 1658-1663.

Schins RPF, Schilderman PAEL, Borm PJA. Oxidative DNA-damage in peripheral blood lymphocytes of coal workers. Int Arch Occup Environ Health 1995; 67: 153-7.

Schins RPF, Borm PJA. Epidemiological evaluation of release of monocyte TNF- $\alpha$ as an exposure and effect marker in pneumoconiosis: a five year follow up study among coal workers. Occup Environ Med 1995; 52: 441-50.

Schins RPF, Lamers RJS, Préat B, Borm PJA. Evaluation of serum procollagen type III peptide as an exposure marker in retired coal workers. Int Arch Occup Environ Health 1995; 66: 413-9.

Schins RPF, Derhaag TJJM, de Jong J, Bast A, Borm PJA. Serum total radical-trapping antioxidant parameter (TRAP) in coal workers. Clin Chem 1994; 40: 1470-2.

Schins RPF, Borm PJA. Serum procollagen type JII peptide in coal worker's pneumoconiosis: A five year follow-up study. Exp Lung Res 1994; 20: 445-55.

Jorna THJM, Schins RPF, Lenaerts L, Derhaag TJJM, Wouters EFM, Borm PJA. Airflow obstruction and monocyte TNF release in coal workers. Exp Lung Res 1994; 20: 421-31.

Lamers RJS, Schins RPF, Wouters EFM, van Engelshoven JMA. High Resolution Computed tomography of the lungs in coal miners with a normal chest radiograph. Exp Lung Res 1994; 20: 411-9.

Marsh JP, Mossman BT, Driscoll KE, Schins RPF, Borm PJA. Effects of aramid, a high strength synthetic fiber on respiratory cells in vitro. Drug Chem Toxicol 1994; 17: 75-92.

Schins RPF, Gaudichet A, Jaurand M-C. A method to obtain a well-defined fraction of respirable aramid fibers. Environ Health Perspect 1993; 101: 414-6.

Borm PJA, Schins RPF. Lymphocyte subsets in subjects exposed to asbestos: changes in circulating natural killer cells. Br J Ind Med 1993; 50 : 575 (Letter).

Borm PJA, Schins RPF, Janssen YMW, Lenaerts L. Molecular basis for differences in susceptibility to coal workers' pneumoconiosis. Toxicol Letters 1992; 64/65: 767-72.

\section{Bookchapter}

Schins RPF, Borm PJA, Lenaerts L. Tumor necrosis factor (TNF) and progression of coal workers' pneumoconiosis in retired coal miners. In: Mohr U, Dungworth DL, Mauderly JL, Oberdörster G (Eds). Toxic and Carcinogenic Effects of Solid Particles in the Respiratory Tract. ILSI Press, Washington,1994, pp. 423-7. 


\section{Abstracts}

Schins RPF, Gosset Ph, Wallaert B, Borm PJA. Tumor necrosis factor- $\alpha$ and transforming growth factor- $\beta$ as biomarkers of coal dust exposure and pneumoconiosis. 6 th International meeting on the toxicology of natural and man-made fibrous and non-fibrous cells, Sept 15-18, 1996, Lake Placid, New York USA.

Knaapen AM, Schins RPF, Godschalk RWL, Borm PJA. DNA adducts and oxidative damage in lung epithelial cells coincubated with neutrophils and benzo[a]pyrene. 6th International meeting on the toxicology of natural and man-made fibrous and non-fibrous cells, Sept 15-18, 1996, Lake Placid, New York USA.

Schins RPF, van Hartingsveldt B, Willemse B, Borm PJA. Ex vivo cytokine release from whole blood. A routine method for health effect screening. Hum Exp Toxicol, in press.

Schins RPF, Keman S, Borm PJA. Blood antioxidants and coal dust induced respiratory disorders. A multi-marker approach. Am J Respir Crit Car Med 1996; 153(4): A473.

Keman S, Willemse B, Schins RPF, Guevarra L, Tollerud DJ, Borm PJA. Blood cytokines as markers of respiratory impairment in chemical workers. Am J Respir Crit Car Med 1996; 153(4): A794.

Borm PJA, Godschalk RWL, Knaapen AM, Zwingmann IH, Schins RPF, Driscoll KE. Neutrophils amplify benzo(a)pyrene-DNA adduct formation in alveolar epithelial cells. Am J Respir Crit Car Med 1996; 153(4): A511.

Schins RPF, Préat B, Borm PJA. Accurate exposure assessment: a prerequisite to evaluate biological markers. Epidemiology 1995; 6: S105.

Schins RPF, Schilderman PAEL, Borm PJA. Oxidative DNA damage in peripheral blood lymphocytes of coal workers. Am J Respir Crit Car Med 1995; 151(4): A569.

Borm P, Godschalk R, Schins R, Zwingmann I, van Schooten F-J. Granulocyte-mediated effects on B(a)P-DNA-adducts and cytokine release in lung target cells. Int J Hyg Environ Med 1995; 198: 3-4.

Borm PJA, Schins RPF, Derhaag T. Grain-dust induced peripheral inflammatory responses in blood in the absence of airway obstruction. Epidemiology 1995; 6: S117.

Keman S, Schins RPF, Lenaerts L, Borm PJA. 10-year longitudinal changes in FEV1 of coal miners are related to later progression of CWP and TNF-alpha production by blood monocytes. Am J Respir Crit Car Med 1995; 151(4): A710.

Schins RPF, Schilderman PAEL, Vankan APH, van Golde MCG, Borm PJA. Oxidative DNA damage in peripheral blood lymphocytes of coal workers. VIIIth International Colloquium on Pulmonary Fibrosis, Oct. 16-19, 1994, Dijon, France.

Schins RPF, Lenaerts L, Borm PJA. Tumor necrosis factor and serum type III procollagen as biological markers in Coal Worker's Pneumoconiosis. Int J Hyg Environ Med 1994; 195: 172-3.

Schins RPF, Derhaag TJJM, Préat B, Borm PJA . Ex-vivo release of tumor necrosis factor- $\alpha$ from monocytes is a good predictor of pneumoconiosis in ex-coal workers. Am J Respir Crit Care Med 1994; 149(4): A556.

Borm PJA, Schins RPF, Jorna THJM, Porcher JM. Validation of TNF as a biological marker of exposure, risk or susceptibility in coal dust induced respiratory disorders. VIIIth Intemational Colloquium on Pulmonary Fibrosis, Oct. 16-19, 1994, Dijon, France.

Borm PJA, Schins RPF, Derhaag TJJM, Kant IJ, Jorna THJM. Rapid priming of monocyte TNF- $\alpha$ release in workers exposed to grain dust. Am J Respir Crit Care Med 1994; 149(4): A401.

Schins R, Lenaerts L, Borm P. Markers of individual risk regarding development and progression of coal workers pneumoconiosis. In: Proceedings of the First Amold O. Beckman/IFCC. European Conference on Environmental Toxjcology "Biomarkers of Exposure" . Munich 16-18 June 1993, Germany.

Schins RPF, Borm PJA. Serum procollagen type III peptide in Coal Worker's Pneumoconiosis: A five year follow-up study. In: Proceedings of the Symposium "Coal Dust Induced Respiratory Disorders", Maastricht 8 Oct, 1993, the Netherlands. 
Borm PJA, Schins RPF. Tumor necrosis factor and serum type III procollagen as marker for progression in coal workers' pneumoconiosis. A 5 year follow-up study. In: Proceedings of the 5th NATO International workshop on the effects of mineral dusts on cells. Cellular and molecular effects of mineral and synthetic dusts and fibres. Paris 11-13 October 1993.

Dong HY, Drion N, Schins R, Yegles M, Kheuang L, Renier A, Brochard P, Jaurand MC. In vitro response of rat pleural mesothelial cells to respirable p-aramid samples. In: Proceedings of the 5 th NATO International workshop on the effects of mineral dusts on cells. Cellular and molecular effects of mineral and synthetic dusts and fibres. Paris 11-13 October 1993.

Lamers RJS, Schins RPF, Borm PJA, Wouters EFM, van Engelshoven JMA. Spirometrically controlled lung densitometry by high-resolution computer tomography (HRCT) in coal miners. In: Proceedings of the Symposium "Coal Dust Induced Respiratory Disorders", Maastricht 8 Oct, 1993, the Netherlands.

Borm PJA, Schins RPF, Jorna THJM, Janssen YMW. Biological markers as indicators of exposure, risk, and susceptibility in coal dust induced respiratory disorders. In: Proceedings of the Symposium "Coal Dust Induced Respiratory Disorders", Maastricht 8 Oct, 1993, the Netherlands.

Jorna THJM, Schins RPF, Lenaerts L, Derhaag TJJM, Wouters EFM, Borm PJA. Airflow obstruction in retired coal miners is related to tumor necrosis factor alpha production by blood monocytes. In: Proceedings of the Symposium "Coal Dust Induced Respiratory Disorders", Maastricht 8 Oct, 1993, the Netherlands.

Jorna THJM, Borm PJA, Lenaerts L, Schins RPF, Derhaag T, Wouters EFM. Airway obstruction in retired coal workers is present at early stages of pneumoconiosis. Am Rev Respir Dis 1993; 147: A902.

Borm PJA, Schins RPF, Préat B, Lenaerts L. A five year follow-up of the TNF release and serum type III procollagen as biological markers for pneumoconiosis. In: Proceedings of the 4 th International Inhalation Symposium "Toxic and Carcinogenic Effects of Solid Particles in the Respiratory Tract", 1-5 March 1993 Hannover, Germany.

Schins RPF, Lenaerts L, Borm PJA. Validation of biological markers to detect coal workers' pneumoconiosis (CWP). a five-year follow-up study. 7th International Colloquium on Puimonary Fibrosis. September 20-23, 1992, Cambridge, UK.

\section{Publication in Dutch}

Evelo CTA, Palmen NGM, Borm PJA, Schins RPF, Zondag R, Pisters R, Leummens M (1991). Fysiologisch gebaseerd farmacokinetiek model (PB-PK model) voor 1,3-butadieen. Rapport opgesteld in opdracht van DSM special products, DSM-Limburg, Geleen. 
